\title{
Fully-succinct Publicly Verifiable Delegation from Constant-Size Assumptions
}

\author{
Alonso González ${ }^{* 1}$ and Alexandros Zacharakis ${ }^{\dagger 2}$ \\ ${ }^{1}$ Toposware Inc. \\ ${ }^{2}$ Universitat Pompeu Fabra, Barcelona, Spain \\ alonso.gonzalez@toposware.com, alexandros.zacharakis@upf.edu
}

September 17, 2021

\begin{abstract}
We construct a publicly verifiable, non-interactive delegation scheme for any polynomial size arithmetic circuit with proof-size and verification complexity comparable to those of pairing based zk-SNARKS. Concretely, the proof consists of $O(1)$ group elements and verification requires $O(1)$ pairings and $n$ group exponentiations, where $n$ is the size of the input. While known SNARK-based constructions rely on non-falsifiable assumptions, our construction can be proven sound under any constant size $(k \geq 2) k$-Matrix Diffie-Hellman $(k-\mathrm{MDDH})$ assumption. However, the size of the reference string as well as the prover's complexity are quadratic in the size of the circuit. This result demonstrates that we can construct delegation from very simple and well-understood assumptions. We consider this work a first step towards achieving practical delegation from standard, falsifiable assumptions.

Our main technical contributions are first, the introduction and construction of what we call "no-signaling, somewhere statistically binding commitment schemes". These commitments are extractable for any small part $x_{S}$ of an opening $x$, where $S \subseteq[n]$ is of size at most $K$. Here $n$ is the dimension of $x$ and $x_{S}=\left(x_{i}\right)_{i \in S}$. Importantly, for any $S^{\prime} \subseteq S$, extracting $x_{S^{\prime}}$ can be done independently of $S \backslash S^{\prime}$. Second, we use these commitments to construct more efficient "quasi-arguments" with no-signaling extraction, introduced by Paneth and Rothblum (TCC 17). These arguments allow extracting parts of the witness of a statement and checking it against some local constraints without revealing which part is checked. We construct pairing-based quasi arguments for linear and quadratic constraints and combine them with the low-depth delegation result of González et. al. (Asiacrypt 19) to construct the final delegation scheme.
\end{abstract}

\footnotetext{
*This work was done while the author was part of LIP laboratory at the ENS de Lyon, France

${ }^{\dagger}$ Research supported by fellowships from la Caixa Foundation (ID 100010434). The fellowship code is LCF/BQ/DI18/11660053. Funding is also from the European Unions Horizon 2020 research and innovation program under the Marie Skodowska-Curie grant agreement No. 713673.
} 


\section{Contents}

1 Introduction $\quad 1$

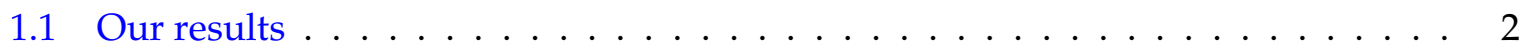

2 Technical Overview 4

2.1 No-Signaling Somewhere Statistically Binding Commitments/Hashing . . . . . . 4

2.1.1 SSB Commitments with Oblivious Trapdoor Generation. . . . . . . . . . . 5

2.1.2 Constructing Oblivious SSB Commitments. . . . . . . . . . . . . . 6

2.2 Pairing-based Quasi-Arguments . . . . . . . . . . . . . . . . 8

2.2 .1 Preliminaries . . . . . . . . . . . . . . . . . . . 9

2.2.2 Oblivious Trapdoor Generation for Quasi-Arguments . . . . . . . . . . . . 11

2.2.3 Quasi-Arguments of Membership in a Linear Space . . . . . . . . . . . . . 12

2.2.4 Quasi-Argument of Hadamard Products . . . . . . . . . . . . . . . . . 13

2.3 From our Quasi-Arguments to Delegation. . . . . . . . . . . . . . . . . . . 15

2.3.1 Delegating bounded space TM/bounded width circuits . . . . . . . . . 15

2.3.2 Overcoming the dependence on space/width. . . . . . . . . . . . 18

2.4 NIZK, SNARKs and Compact NIZK . . . . . . . . . . . . . . . . . . 20

3 Preliminaries 21

3.1 Notation . . . . . . . . . . . . . . . . . . . . . 21

3.2 Cryptographic Assumptions . . . . . . . . . . . . . . . . . . . . . . 22

3.3 Arguments of Knowledge Transfer . . . . . . . . . . . . . . . . . . . 23

4 No-Signaling Somewhere Statistically Binding Commitments 24

4.1 Algebraic SSB Commitments. . . . . . . . . . . . . . . . . . . 26

4.2 Somewhere Statistically Binding Commitments with Oblivious Trapdoor Gen-

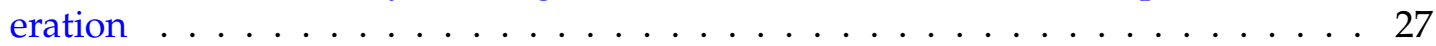

4.3 Kronecker Product of two SSB commitments . . . . . . . . . . . . . . . . . . 31

5 Quasi-Arguments with Preprocessing 34

5.1 Arguments with No-signaling extraction and Oblivious CRS Generation . . . . . 35

5.2 Succinct Pairing Based Quasi-Arguments . . . . . . . . . . . . . . . . . 38

5.2.1 Quasi Arguments of Membership in Linear Spaces . . . . . . . . . . . 38

5.2.2 Quasi-Arguments for Hadamard Products. . . . . . . . . . . . . . . . . . . 55

6 Delegation for Arithmetic Circuit Evaluation $\quad 62$

6.1 The Scheme . . . . . . . . . . . . . . . . . . . . . . 62

6.2 Proof of Security . . . . . . . . . . . . . . . . . . . . . 63

7 Applications $\quad 66$

7.1 NIZK arguments for NP. . . . . . . . . . . . . . . . . . . . 67

$\begin{array}{ll}\text { A Delayed proof from Section } 3.3 & 75\end{array}$ 


\section{Introduction}

In a delegation scheme, a verifier with limited computational resources (a mobile device for example) wishes to delegate a heavy but still polynomial computation to an untrusted prover. The prover, with more computational power but still of polynomial time, computes a proof which the verifier accepts or rejects. Given the limitations of the verifier, the proof should be as short as possible and the verification process should consume as few computational resources as possible. Additionally, the construction of the proof should not be much costlier than performing the computation itself.

A delegation scheme can be easily constructed from a zero-knowledge Succinct NonInteractive Argument of Knowledge (zk-SNARK) for NP. Schemes like [GGPR13; Gro16] are very appealing in practice because a proof consists of only a constant number of group elements and verification requires the evaluation of a constant number of pairings. ${ }^{1}$ The downside is that these zk-SNARKs are based on strong and controversial assumptions such as the knowledge of exponent assumption or the generic group model.

Such assumptions are called non-falsifiable because there is no way of efficiently deciding whether an adversary breaks the assumption or not. In such assumptions, the adversary is treated in a non black box way and the assumption argues about how an adversary performs a computation instead of what computation it cannot perform. Since zk-SNARKs can handle even NP computations, soundness becomes an essentially non-falsifiable property where one needs to decide whether an adversary produces a true or false statement without any witness but only with a very short proof. Gentry and Wichs [GW11] proved that zk-SNARKs for NP are (in a broad sense) impossible to construct without resorting to non-falsifiable assumptions.

While this impossibility result justifies the use of such assumptions for non-deterministic computation, this is not the case for delegation of computation which only considers deterministic computation. Indeed, in this case, soundness becomes an efficiently falsifiable statement: determining whether the adversary breaks soundness simply requires to evaluate the delegated polynomial computation on some input $x$ and check whether it is accepting or rejecting. Actually, getting delegation from falsifiable assumptions is easy in general: let $\Pi$ be a SNARK for NP. For a binary relation $R$, the assumption " $\Pi$ is sound for $R$ " is in general non-falsifiable since checking membership in the corresponding language is hard and the SNARK proof does not help as shown by [GW11]. On the contrary, for a relation $R$ in $P$, the assumption becomes falsifiable since one can efficiently compute $R(x)$. Nevertheless, the important issue is to consider the quality of the assumption in place since the assumption "the proof system is sound" is tautological. Ideally, we should rely on simple and well understood assumptions without sacrificing other desirable properties.

Almost all known constructions that base their soundness on falsifiable assumptions (or even no assumptions at all) come with some compromises: they (1) are not expressive enough to capture all polynomial time computation [KPY18; GR19; CCH+19; JKKZ20] (2) are interactive [GKR08; RRR16], (3) are designated verifier [KRR13; KRR14; KP16; BHK17; BKK+18] or (4) rely on strong (yet falsifiable) assumptions related to obfuscation [CHJV15; KLW15; BGL+15; ACC +16 ; CCC +16 ] or multi-linear maps [PR17].

An exception to this is a construction of Kalai et al. [KPY19] of a delegation scheme for any poly-time computation based on a newly introduced $q$-size assumption in bilinear groups. The size of the assumption is $q=\log T$ and $T$ is the time needed to perform the computation. As for efficiency, the size of the proof is polylog $(T)$ group elements which becomes poly $(\kappa)$ if $T \leq 2^{\kappa}$.

However, in spite of the recent progress, there's still a gap in the proof size and verification with respect to the most efficient known constructions, namely those based on paring based

${ }^{1}$ Note that zero-knowledge is not necessary. 
zk-SNARKs.

\subsection{Our results}

In this work we consider the question "what are the simplest assumptions that imply publicly verifiable, non-interactive delegation of computation"? Here "simple" should be interpreted as falsifiable and well understood. Having practicality in mind as well, we would also want a delegation scheme that competes in efficiency with the most efficient constructions to date, namely those that are based on non-falsifiable assumptions.

The main contribution of this work is the construction of a fully-succinct, non-interactive, publicly verifiable delegation scheme from any $k$-Matrix Diffie-Hellman assumption ( $k$-MDDH) for $k \geq 2$, as for example the decisional linear assumption (DLin) [BBS04]. In the more efficient setting of asymmetric groups, soundness can be based on the natural translation of symmetric DLin where the challenge is encoded in both groups (the SDlin assumption of [GHR15b]). Here by fully-succinct we mean that the proof size is linear in the security parameter and verification requires a linear number of operations (whose complexity depends only on the security parameter) in the size of the input of the computation. We achieve these goals but with the drawback that the prover computation and the size of the crs are quadratic in the size of the circuit. Our main contribution is summarized in the next (informal) theorem.

Theorem 1. (Informal). There exists a non-interactive, publicly verifiable delegation scheme for any polynomial size circuit $C$ with $n$-size input that is adaptively sound under any $k-M D D H$ assumption for $k \geq 2$ with the following efficiency properties: the crs size is poly $(\kappa)|C|^{2}$, prover complexity is $\operatorname{poly}(\kappa)|C|^{2}$, proof size is poly $(\kappa)$ and verification complexity is poly $(\kappa) n$.

Our construction is also concretely efficient as far as proof size and verification complexity are concerned. The proof comprises of $10+8$ group elements of an asymmetric bilinear group and verification requires $n$ exponentiations plus 36 evaluations of the pairing function, where $n$ is the size of the input. The attractive concrete efficiency is achieved due to the structurepreserving nature $[\mathrm{AFG}+16]$ of our construction. This notion captures that all algorithms solely perform group operations, namely they are algebraic, and there is no need to encode cryptographic primitives such as hash functions or pairings as arithmetic circuits, a process that is very inefficient in practice.

This result demonstrates two things. First, delegation of computation can be based on very simple, standard assumptions. Second, its structure preserving nature hints to the plausibility of practically efficient delegation schemes comparable in efficiency with the ones based on SNARKs, but under simple, standard assumptions. In table 1 we present a comparison of our delegation of computation construction with other pairing based schemes.

No-Signaling SSB Commitments and Succinct Pairing-based Quasi-Arguments. We follow and extend the ideas of Paneth and Rothblum [PR17] and Kalai et al. [KPY19] for constructing delegation schemes for poly-time computations from what they called quasi-arguments of knowledge with no-signaling extractors. First, we formalize a similar notion for commitment schemes and show that the somewhere statistically binding (SSB) commitments of [GHR15b; FLPS20] are no-signaling when they also have what we call an "oblivious trapdoor generator". Second, we use the no-signaling SSB commitments to construct more efficient constant-sized quasi-arguments of knowledge for linear and quadratic relations. We achieve this by combining SSB commitments with the very efficient quasi-adaptive non-interactive zero-knowledge arguments for linear [JR13; LPJY13; JR14; KW15] and quadratic relations [GHR15b; DGP+19]. To this aim, we also show that the QA-NIZK arguments can be easily modified to have no-signaling extractors under standard assumptions. 
Table 1: Comparison between different pairing based delegation schemes and our results.

\begin{tabular}{||cccccc||}
\hline & Language & Verification & Proof size & CRS size & Assumption \\
\hline \hline [GGPR13][Gro16] & $\mathrm{AC}$ & $n \mathrm{e}+O(1) \mathrm{p}$ & $O(\kappa)$ & $O(|C| \kappa)$ & Non Falsifiable \\
[KPY19] (base case) & $\mathrm{RM}$ & $n \mathrm{e}+\operatorname{poly}(\log d) \mathrm{p}$ & $O(\kappa \log d)$ & $O((n+d) \kappa)$ & $\log d$-Assumption \\
[GR19] & $\mathrm{AC}$ & $n \mathrm{e}+O(d) \mathrm{p}$ & $O(d \kappa)$ & $O(|C| \kappa)$ & $s$-Assumption \\
This work & $\mathrm{AC}$ & $n \mathrm{e}+O(1) \mathrm{p}$ & $O(\kappa)$ & $O\left(|C|^{2} \kappa\right)$ & DLin/SDLin \\
\hline
\end{tabular}

Verification is given in number exponentiations (e) and pairings (p). $d$ is the circuit depth/number of steps of a computation, $n$ the number of inputs, $s$ the circuit width/computation space and $|C|$ the circuit size. AC stands for "Arithmetic Circuit" and RM for "RAM Machine". For [KPY19] we only consider the "base case" and not the "bootstrapped" constructions, because bootstrapping adds a considerable overhead and is thus incomparable in terms of group operations. We stress out, however, that the crs size of the bootstrapped construction is sublinear in the time of the computation.

Applications to NIZK. Our construction can be turned into a NIZK argument for NP of size $n+O(1)$ group elements -namely $O(n \kappa)$ proof size- under the same assumptions where $n$ is the number of public an secret inputs of the circuit. In table 2 we provide a comparison of our NIZK construction and the literature. Using standard techniques, the argument implies compact NIZK for NP with proof size $O(n)+$ poly $(\kappa)$. That is, the size of the proof is proportional to the size of the input and the security parameter only gives an additive overhead. In comparison, the state of the art is $O(|C|)+\operatorname{poly}(\kappa)$ for poly-sized boolean circuits and $O(n)+\operatorname{poly}(\kappa)$ for log-depth boolean circuits [KNYY19; KNYY20]. We note that a similar result can be obtained by [KPY19], albeit with a stronger assumption.

Table 2: Comparison between different pairing based NIZK schemes and our results.

\begin{tabular}{||cccccc||}
\hline & Language & Verification & Proof size & CRS size & Assumption \\
\hline \hline [GOS06] & $\mathrm{AC}$ & $O(|C|) \mathrm{p}$ & $O(|C| \kappa)$ & $O(\kappa)$ & SXDH \\
[GGPR13][Gro16] & $\mathrm{AC}$ & $O(1) \mathrm{p}$ & $O(\kappa)$ & $O(|C| \kappa)$ & Non Falsifiable \\
[GR19] & $\mathrm{BC}$ & $O(n+d) \mathrm{p}$ & $O((n+d) \kappa)$ & $O(|C| \kappa)$ & s-Assumption \\
[KNYY20] & $\mathrm{NC}{ }^{1}$ & $O(|C|)$ poly $(\kappa)$ & $n$ poly $(\kappa)$ & poly $\left(|C|, \kappa, 2^{d}\right)$ & DLin \\
This work & $\mathrm{BC}$ & $O(n) \mathrm{p}$ & $n O(\kappa)$ & $O\left(|C|^{2} \kappa\right)$ & DLin/SDLin \\
\hline
\end{tabular}

Verification is given in number of pairings p. $d$ is the circuit depth, $n$ the number of (public and secret) inputs, $s$ the circuit width and $|C|$ the circuit size. AC stands for "Arithmetic Circuit" and BC for "Boolean Circuit".

Our argument can be also used to construct zk-SNARKs from quantitatively weaker assumptions than the state of the art. Indeed, the strongest assumption used in zk-SNARKs such as [GGPR13; Gro16] is a knowledge assumption which states that an adversary computing some elements of a bilinear group, satisfying a particular relation, must know their discrete logarithms. ${ }^{2}$ Such assumption is used to extract an assignment to each of the circuit wires. The "size" of such assumption is proportional to the number of extracted values, which in this case is the size of the circuit. Since our argument only requires the reduction to know the input of the circuit, we can rely on a knowledge assumption only for extracting the input. As a consequence the size of the assumption is drastically shortened. Since these assumptions are

\footnotetext{
${ }^{2}$ Actually, the adversary must know a representation of these values as a linear combination of a set of group elements that she receives as input.
} 
stronger as the size of the assumption increases and given that we lack good understanding of them, it is always safer to rely on shorter assumptions. Also, weaker assumptions translates to better concrete efficiency by using smaller security parameters. ${ }^{3}$

\section{Technical Overview}

To construct the delegation scheme we follow a commit-and-prove approach, which means that we first commit to the witness (the satisfying assignment of wires in a circuit) and then show that this witness satisfies some relation. We use somewhere statistically binding (SSB) commitments as those used in [GHR15b; GR16; FLPS20] and show that they satisfy a no-signaling extraction property. Then, we do the same for the so called quasi-adaptive NIZK arguments for linear spaces [JR13; LPJY13; JR14; KW15] and for quadratic relations [GHR15b; DGP+19]. From these primitives we can construct delegation for bounded-space computations/bounded width circuits with proof-size independent of the depth of the computation by following the techniques of [PR17; KPY19]. To get a succinct proof-size, in addition to the "depth compression" we must also perform a "width compression". To this end, we use ideas from the delegation scheme for bounded depth computations of González and Ràfols [GR19] and remove the necessity of a $q$-assumption to rely solely on constant size assumptions. To combine both "compressions" efficiently we exploit the fact that [GR19] is structure preserving and the verifier is a bounded width circuit. In the next sections we present these techniques.

\subsection{No-Signaling Somewhere Statistically Binding Commitments/Hashing}

Somewhere statistically binding (SSB) hashing/commitments ${ }^{4}$ were introduced by Hubacek and Wichs [HW15] and then improved by [OPWW15], and have been used for constructing efficient NIZK proofs [GHR15b; GR16] as well as ring signatures [BDH+19].

An SSB commitment scheme is a generalization of dual mode commitments [GS08] where the commitment key can be sampled from many computationally indistinguishable distributions, each of which is making the commitments statistically binding for a number of $K$ coordinates of the commited value. That is, when commiting to a vector $m=\left(m_{1}, \ldots, m_{n}\right)$ with a commitment key $c k_{S}$ associated with a set $S \subseteq[n]$ of size at most $K$, no (even computationally unbounded) adversary can compute a commitment $c$ and two valid openings $m, m^{\prime}$ such that for some $i \in S$ it holds that $m_{i} \neq m_{i}^{\prime}$, except with negligible probability. Importantly, the size of the commitment $c$ should be independent of $n$ but may depend on the value $K$.

Known SSB commitments constructions are also extractable ${ }^{5}$, that is, there exists an efficient algorithm that has some trapdoor information associated with $c k_{S}$ and can efficiently extract from a commitment $c$ a valid opening $\left(m_{i}\right)_{i \in S}$. Note that the notion of a "valid opening" is well-defined due to the statistical binding property on the set $S$.

We argue that the SSB extractor has many similarities with the no-signaling extractors of [PR17; KPY19]. First, we briefly recall what a no-signaling extractor is in the context of quasi arguments of knowledge. A quasi argument is a proof system for a relation that defines

\footnotetext{
${ }^{3}$ We note, however, that in the case of non-falsifiable assumptions it not clear how an appropriate security parameter should be chosen.

"Through this paper we will refer to "commitments" while technically they are "hashes". We do so because in the context of NIZK proofs is traditional to commit to the witness and then prove that the committed value satisfy some relation. However, since we are less interested in zero-knowledge, the randomness of such commitments is 0 (or fixed/inexistent) and we end up with hashes.

${ }^{5}$ In the context of bilinear groups, we can consider $f$-extraction where one only extracts $f$ applied to the witness. In particular, it is usual to consider $f$ the (one-way) function that maps elements in $\mathbb{Z}_{p}$ to one of the base groups $\mathbb{G}_{1}$ or $\mathbb{G}_{2}$. This is the notion of extractability we use in this work and is enough to obtain our results.
} 
some local constraints on the statement/witness pair. The requirement is that there exists a no signaling extractor that allows extracting a part of the witness from a verifying proof that is locally correct. Furthermore, each part of the extracted local witness can be in a sense extracted independently. This is formalized by requiring that extracting local witness $w_{S}$ for a set $S$ and restricting it to the variables $S^{\prime} \subseteq S$ is computationally indistinguishable from extracting $w_{S^{\prime}}$ for the set $S^{\prime}$. As we shall see shortly, this property is extremely useful when constructing delegation schemes.

In the case of SSB commitments, extractability of the local opening is just a local soundness guarantee. Additionally, indistinguishability of the commitment keys is a weaker form of the no-signaling property. Indeed, a no-signaling extractor must produce commitment keys which are indistinguishable for the various possible extractable sets. Otherwise a distinguisher for sets $S, S^{\prime}$ can be used for wining in the no-signaling game even without the extracted value. Nevertheless, this alone does not satisfy the no-signaling property: some information about the positions where the crs is programmed to extract might be revealed by (parts of) the extracted local openings.

We strengthen the indistinguishability property of the distributions of the commitment keys of SSB commitments to give them a no-signaling flavour. Roughly speaking, we require that the distributions of the commitment keys are computationally indistinguishable even if the adversary has access to local openings associated with a set $S^{\prime}$ of committed values. These local openings trivially reveal information about the set $S^{\prime}$ but we require that they do not leak information about the values outside of $S^{\prime}$. That is, for any sets $S^{\prime} \subseteq S$ of size at most $K$, the commitment keys $c k_{S}, c k_{S^{\prime}}$ are computationally indistinguishable even if we allow the distinguisher access to local openings of $S^{\prime}$.

Remark (Connection with PIR). Somewhere statistically binding commitments/hashing is closely related with single server Private Information Retrieval Schemes (PIR) when the SSB commitment is also extractable. Indeed, we can think of the commitment key for an index $i$ of the SSB as a PIR query and the commitment/hash as the PIR answer. Then, one can decode the PIR query using the trapdoor associated with the commitment key. In our work, the SSB commitments we use are different from PIRs in three ways: (1) we do not extract the PIR answers, but we $f$-extract, specifically we extract encodings of messages in a group but not their discrete logarithms, (2) we directly use SSBs with locality greater than one instead of making parallel PIR queries to improve concrete efficiency and (3) the size of the commitment key is proportional to the size of the commited values, while in PIRs the query should be small compared to the database size. Furthermore, we exploit in a non-black box way the properties as well as the algebraic structure of the SSB commitments to compose them with other protocols, such as group based quasi-adaptive non-interactive zero knowledge arguments.

\subsubsection{SSB Commitments with Oblivious Trapdoor Generation.}

We define a stronger notion for SSB commitment schemes, oblivious trapdoor generation, which implies the no-signaling property. This notion is easier to work with in our particular constructions.

Intuitively, this notion captures that there exists a different, oblivious key generation algorithm that can generate the commitment key for $S$ and a trapdoor for a subset $S^{\prime} \subseteq S$ obliviously of $S \backslash S^{\prime}$ for any subset $S^{\prime}$ of the larger set $S$ of binding coordinates. More concretely, the oblivious key generation algorithm takes as input a commitment key $c k_{S}$ binding at $S$ and the description of a subset $S^{\prime} \subseteq S$ and outputs an identically distributed key together with a trapdoor for extracting values in the small set $S^{\prime}$. We emphasize that this algorithm does not take as input neither the description of $S$ nor the trapdoor associated with it. Intuitively, the key generation 
algorithm is oblivious of $S \backslash S^{\prime}$ (it might even be that $S \backslash S^{\prime}=\emptyset$ ) due to the indistinguishability of commitment keys associated with different sets, in this case $S$ and $S^{\prime}$.

This property implies no-signaling commitments. Indeed, this follows easily since (1) by the index set hiding property the commitment key itself does not reveal any information about $S \backslash S^{\prime}$ and (2) we can use the oblivious key generation algorithm to create a trapdoor for extracting the smaller set without skewing the distribution of the commitment key. The latter property means essentially that we are given an oracle to extract the smaller set (by computing the trapdoor for an identically distributed key) which is exactly what the no-signaling property captures.

\subsubsection{Constructing Oblivious SSB Commitments.}

We next describe how to construct efficient SSB commitments with oblivious trapdoor generator. A natural way to construct oblivious SSB commitment with locality parameter $K$ is to concatenate $K$ SSB commitments with locality parameter 1 . Consider a set $S=\left\{s_{1}, \ldots, s_{t}\right\}$ for some $t \leq K$. We can construct a commitment key associated with $S$ by computing $t$ commitment keys/trapdoor pairs $\left(c k_{1}, \tau_{1}\right), \ldots,\left(c k_{t}, \tau_{t}\right)$ for sets $\left\{s_{1}\right\}, \ldots,\left\{s_{t}\right\}$, complementing with $K-t$ keys for $\emptyset$ if necessary. To commit to some $x \in \mathcal{M}^{n}$, where $\mathcal{M}$ is the message space of the commitment, one simply computes $c_{1}=\operatorname{Com}_{c k_{1}}(x), \ldots, c_{K}=\operatorname{Com}_{c k_{K}}(x)$. Extraction of each $x_{s_{i}}$ is done using $c_{s_{i}}$ and the trapdoor $\tau_{s_{i}}$, independently of the others. The oblivious extractor on input the commitment keys for some unknown $S$ and the description of $S^{\prime} \subseteq S$ just re-samples the commitment keys for $S^{\prime} .{ }^{6}$ Since it doesn't matter if the trapdoors for positions $i \notin S^{\prime}$ are not known, this trivial extractor can obliviously generate the trapdoor $\left\{\tau_{i}: i \in S^{\prime}\right\}$.

While this generic construction is enough, we can construct more efficient ones if we consider specific instantiations. More specifically, as we present next, we can have more efficient instantiations (roughly half commitment size compared to the generic one) in the case of commitments derived from the Pedersen commitment scheme.

Notation. We first need to introduce some notation. When $S \subseteq[n]$ we denote with $\bar{S}$ the set $[n] \backslash S$. For a vector $\boldsymbol{x}$ (resp. matrix $\mathbf{G}$ ) we denote $\boldsymbol{x}_{S}=\left(x_{i}\right)_{i \in S}$ (resp. $\mathbf{G}_{S}=\left(\boldsymbol{g}_{i}\right)_{i \in S}$ where $\boldsymbol{g}_{i}$ is the $i$-th column of $\mathbf{G}$ ). Finally, we use implicit notation for groups. That is, given a group $\mathbb{G}$ and a fixed generator $\mathcal{P}$ we denote with $[r]$ the element $r \mathcal{P}$. For vectors and matrices $\boldsymbol{a}, \mathbf{A}$ respectively, we denote with $[a],[\mathrm{A}]$ the natural embeddings of $\boldsymbol{a}, \mathbf{A}$ to $\mathbb{G}$.

For vectors $\boldsymbol{a}, \boldsymbol{b}$, we denote $\boldsymbol{a} \circ \boldsymbol{b}=\left(a_{i} b_{i}\right)_{i}$ the Hadamard product of them, and for matrices $\mathbf{A}=\left(a_{i, j}\right)_{i, j}, \mathbf{B}$ we denote $\mathbf{A} \otimes \mathbf{B}=\left(a_{i, j} \mathbf{B}\right)_{i, j}$ their Kronecker product. We will be using the mixed-product property of kronecker products, which says that $(\mathbf{A} \otimes \mathbf{B})(\mathbf{C} \otimes \mathbf{D})=(\mathbf{A C}) \otimes(\mathbf{B D})$ whenever A, B, C, D have the appropriate dimensions.

Efficient SSB Commitments. We next present an oblivious SSB construction based on the Pedersen commitment scheme. This construction was implicit in [GHR15b] and later generalized in [FLPS20]. Later we will see that it also satisfies the stronger notion of oblivious trapdoor generation.

Let $\mathbb{G}$ be a group of size $p$. For message space $\mathbb{Z}_{p}^{d}$, locality parameter $K \in \mathbb{N}$ and a subset $S \subseteq[d]$ of size $t \leq K$, the commitment key is defined as follows: $\mathbf{G}=\left(\mathbf{G}_{S} \mid \mathbf{G}_{\bar{S}}\right) \mathbf{P}$ and

$$
\mathbf{G}_{S} \leftarrow \mathbb{Z}_{p}^{(K+1) \times t}, \quad \mathbf{G}_{0} \leftarrow \mathbb{Z}_{p}^{(K+1) \times(K+1-t)}, 7 \quad \quad \mathbf{} \leftarrow \mathbb{Z}_{p}^{(K+1-t) \times(d-t)}, \quad \mathbf{G}_{\bar{S}}=\mathbf{G}_{0} \mathbf{\Gamma} .
$$

${ }^{6}$ Actually, the oblivious key generation needs to know which of the commitments keys $c k_{1}, \ldots, c k_{K}$ are perfectly binding for $s^{\prime} \in S^{\prime}$. Nevertheless, it should be still oblivious of whether the rest of commitment keys are binding or not. See section 4.2 for more details.

${ }^{7}$ It is not always the case that this matrix is uniform. The actual property needed is that this matrix satisfies 
Matrix $\mathbf{P} \in\{0,1\}^{d \times d}$ is a permutation matrix associated to $S$ such that $\mathbf{P} \boldsymbol{e}_{s_{i}}=\boldsymbol{e}_{i}$, for $i \leq t$ and $\boldsymbol{e}_{i}$ the $i$-th vector of the canonical basis. A commitment to $x \in \mathbb{Z}_{p}^{d}$ is computed as $[c]=[\mathrm{G}] x=$ $\left[\mathbf{G}_{S} \mid \mathbf{G}_{\bar{S}}\right] \mathbf{P} x=\left[\mathbf{G}_{S}\right] x_{S}+\left[\mathbf{G}_{\bar{S}}\right] x_{\bar{S}}$. Note that the columns of $\mathbf{G}_{S}$ are linearly independent from the columns of $\mathbf{G}_{\bar{S}}$ with overwhelming probability, since $\operatorname{Im}\left(\mathbf{G}_{\bar{S}}\right) \subseteq \operatorname{Im}\left(\mathbf{G}_{0}\right)$ and $\left(\mathbf{G}_{S} \mid \mathbf{G}_{0}\right)$ is a basis of $\mathbb{Z}_{p}^{K+1}$ w.o.p. since this corresponds to a uniform matrix of dimensions $K+1 \times K+1$.

This distribution of commitment keys implies that the parts of the input indexed by $S$ go to the space spanned by $\mathbf{G}_{S}$ of dimension $t$, while the rest is mapped to the space spanned by $\mathbf{G}_{0}$ of dimension $K+1-t$. Since $\operatorname{rank}\left(\mathbf{G}_{S}\right)=t$ with overwhelming probability, all the information of $x_{S} \in \mathbb{Z}_{p}^{t}$ can be retrieved from $c$. Even more, there exists an efficiently computable trapdoor $\mathbf{T}_{S} \in \mathbb{Z}_{p}^{(K+1) \times t}$ such that $\mathbf{T}_{S}^{\top} \mathbf{G}_{S}=\mathbf{I}_{t \times t}$ and $\mathbf{T}_{S}^{\top} \mathbf{G}_{\bar{S}}=\mathbf{0}_{t \times(d-t)}$, and hence

$$
\mathbf{T}_{S}^{\top}[c]=\mathbf{T}_{S}^{\top}[\mathrm{G} x]=\mathbf{T}_{S}^{\top}\left[\mathbf{G}_{S} x_{S}+\mathbf{G}_{\bar{S}} x_{\bar{S}}\right]=\left[x_{S}\right] .
$$

To compute $\mathbf{T}_{S}$, it is enough to solve the linear system $\mathbf{T}_{S}^{\top}\left(\mathbf{G}_{S} \mid \mathbf{G}_{0}\right)=\left(\mathbf{I}_{S} \mid \mathbf{0}\right)$ which admits a solution since $\left(\mathbf{G}_{S} \mid \mathbf{G}_{0}\right)$ is a basis of $\mathbb{Z}_{p}^{K+1}$ with overwhelming probability.

Note that this shows also that the commitment is statistically binding in $S$. The indistinguishability of commitment keys can be shown with a tight reduction to the DDH assumption as in [FLPS20].

Oblivious Trapdoor Generation. One of the main technical contributions of this work is an oblivious trapdoor generator for this commitment scheme, which in turns implies that it is no-signaling. Recall that the property requires that there exists an efficient algorithm, called the oblivious key generation algorithm, that receives as input the description of a set $S^{\prime}$ of size $t^{\prime} \leq K$ and a commitment key [G] sampled for being binding at some unknown $S \supseteq S^{\prime}$. The algorithm computes a new commitment key $[\mathbf{H}]$ with the following guarantees: (1) it is statistically close to [G] and (2) we also obtain a trapdoor $\mathbf{T}_{S^{\prime}}$ that allows us to extract local openings for the small set $S^{\prime}$.

Since we know that columns in $S^{\prime}$ are uniformly distributed, we could attempt to sample a uniform matrix $\mathbf{H}_{S^{\prime}} \leftarrow \mathbb{Z}_{p}^{(K+1) \times t^{\prime}}$ and solve the equation $\mathbf{T}_{S^{\prime}}^{\top} \mathbf{H}_{S^{\prime}}=\mathbf{I}_{t^{\prime} \times t^{\prime}}$ for some $\mathbf{T}_{S^{\prime}}$. However, since we don't know the distribution of $\left[\mathbf{G}_{\bar{S}^{\prime}}\right]$ the only hope seems to be to define $\left[\mathbf{H}_{\bar{S}^{\prime}}\right]=\left[\mathbf{G}_{\bar{S}^{\prime}}\right]$

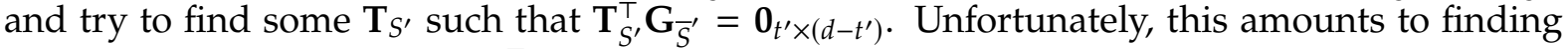
elements in the kernel of $\left[\mathrm{G}_{\bar{S}^{\prime}}\right]^{\top}$ which is in general a computationally hard problem [MRV16].

Instead we make the following observation. Regardless of the distribution of the columns in $S \backslash S^{\prime}$, the $t^{\prime}$ lower rows of $\mathbf{G}_{\bar{S}}$ can be always written as a random linear combination of the first $K+1-t^{\prime}$ rows. That is

$$
\mathbf{G}_{\bar{S}^{\prime}}=\left(\begin{array}{c}
\mathbf{A} \\
\mathbf{R A}
\end{array}\right), \text { where } \mathbf{A} \in \mathbb{Z}_{p}^{K+1-t^{\prime} \times d-t^{\prime}} \text { and } \mathbf{R} \leftarrow \mathbb{Z}_{p}^{t^{\prime} \times K+1-t^{\prime}} .
$$

In this case, if we know the matrix $\mathbf{R}$ in the field, it is possible to compute elements in the kernel of $\mathbf{G}_{\bar{S}^{\prime}}$ by setting

$$
\mathbf{T}_{S^{\prime}}=\left(\begin{array}{c}
-\mathbf{R}^{\top} \mathbf{C} \\
\mathbf{C}
\end{array}\right) \text {, for any } \mathbf{C} \in \mathbb{Z}_{p}^{t^{\prime} \times t^{\prime}}
$$

If additionally, we choose some $\mathbf{C}$ that satisfies $\mathbf{T}_{S^{\prime}}^{\top} \mathbf{H}_{S^{\prime}}=\mathbf{I}_{t^{\prime} \times t^{\prime}}$ we have computed a trapdoor for $S^{\prime}$. This yields a way to compute the rest of the columns: discard the lower $t^{\prime}$ rows of $\mathbf{G}_{\bar{S}}$,

some hardness assumption. Specifically, the index set hiding property reduces to the $\mathcal{G}$-MDDH assumption (see Section 2.2.1 for an informal definition) where $\mathcal{G}$ is the distributions from which we sample $\mathbf{G}_{0}$. When working with symmetric groups, we instantiate using the DLIN assumption. For the sake of simplicity we consider the uniform case in the technical overview. 
sample a uniform matrix $\mathbf{R}$ as above and complete the last rows with the elements $\mathbf{R}[\mathbf{A}]$. Then, using $\mathbf{R}, \mathbf{H}_{S^{\prime}}$ (which are known in the field) find some $\mathbf{C}$ that satisfies the linear equations and use it to define the trapdoor $\mathbf{T}_{S}^{\prime}$.

Lets see in more detail why the previous observation holds. Consider the matrix $\mathbf{G}_{0} \in$ $\mathbb{Z}_{p}^{(K+1) \times(K+1-t)}$ and note that the upper part $\overline{\mathbf{G}}_{0}$ is a uniformly distributed matrix with more rows than columns; hence $\mathbf{R} \overline{\mathbf{G}}_{0}$, for $\mathbf{R} \leftarrow \mathbb{Z}_{p}^{t^{\prime} \times\left(K+1-t^{\prime}\right)}$, is uniformly distributed. This is also valid for all non-binding coordinates since $\mathbf{G}_{\bar{S}}=G_{0} \Gamma$ and then the lower rows follow distribution $\mathbf{R} \overline{\mathbf{G}}_{\bar{S}}$. Next, consider the columns corresponding to the (unknown) binding coordinates $S \backslash S^{\prime}$. The same argument holds: for some uniform $\mathbf{R}^{\prime} \overline{\mathbf{G}}_{S \backslash S^{\prime}}$ is uniform when $\mathbf{R}^{\prime} \leftarrow \mathbb{Z}_{p}^{t^{\prime} \times\left(K+1-t^{\prime}\right)}$. It remains to show that using the same randomness for both column sets, i.e. setting $\mathbf{R}=\mathbf{R}^{\prime}$, does not alter the distribution of the commitment key. Indeed, with overwhelming probability, the columns of $\overline{\mathbf{G}}_{0} \in \mathbb{Z}_{p}^{\left(K+1-t^{\prime}\right) \times(K+1-t)}$ and of $\overline{\mathbf{G}}_{S \backslash S^{\prime}} \in \mathbb{Z}_{p}^{\left(K+1-t^{\prime}\right) \times\left(t-t^{\prime}\right)}$ form a basis of $\mathbb{Z}_{p}^{K+1-t^{\prime}}$, which means that the matrix $\mathbf{R}^{\top}$ can be decomposed into two independent components: $a$ random element in $\operatorname{Im}\left(\overline{\mathbf{G}}_{S \backslash S^{\prime}}^{\perp}\right)$ and another in $\operatorname{Im}\left(\overline{\mathbf{G}}_{0}^{\perp}\right)$. This shows that $\mathbf{R}_{\mathbf{G}_{0}}=\mathbf{R}_{2}\left(\mathbf{G}_{S \backslash S^{\prime}}^{\perp}\right)^{\top} \overline{\mathbf{G}}_{0}$ and $\mathbf{R} \overline{\mathbf{G}}_{S \backslash S^{\prime}}=\mathbf{R}_{1}\left(\mathbf{G}_{0}^{\perp}\right)^{\top} \overline{\mathbf{G}}_{S \backslash S^{\prime}}$ are independent and then $\left(\begin{array}{cc}\overline{\mathbf{G}}_{S \backslash S^{\prime}} & \overline{\mathbf{G}}_{0} \mathbf{\Gamma} \\ \mathbf{R} \overline{\mathbf{G}}_{S \backslash S^{\prime}} & \mathbf{R} \overline{\mathbf{G}}_{0} \mathbf{\Gamma}\end{array}\right)$ is correctly distributed.

\subsection{Pairing-based Quasi-Arguments}

Paneth and Rothblum [PR17] and then Kalai et al. [KPY19] used a weakened version of an argument of knowledge called quasi-argument, as an intermediate step for obtaining a delegation scheme. Quasi arguments are defined for languages that can be expressed as a set of local constraints. Roughly speaking, this means that a witness $w$ for membership of a statement $x$ in a language can be decomposed in parts, namely $\boldsymbol{w}=\left(w_{1}, \ldots, w_{n}\right)$, and for each subset $S \subseteq[n]$, the partial witness $\boldsymbol{w}_{S}$ satisfies some local relations, that is, a predicate $\mathcal{R}\left(x, \boldsymbol{w}_{S}\right)$ holds. For example, in the case of a CNF formula of $n$ variables, the witness is an accepting assignment of the formula and a local constraint with respect to some set $S$ captures that every clause that only has variables $w_{i}, w_{j}, w_{k}$ for $i, j, k \in S$ is satisfied. Note that it can be the case that even unsatisfiable formulas can satisfy all local constraints for families of sets of small size (yet, no global satisfying assignment exists).

Unlike an argument of knowledge, a quasi-argument has only local extraction, meaning that only a small part of the witness of size at most $K$, the locality parameter, is extracted. This is formalized by means of an extractor which on input a set $S \subseteq[n]$ of size at most $K$, where $n$ is the size of the witness, programs a crs so that it can later extract positions of the witness defined by $S$. Central to quasi-arguments is the notion of no-signaling local extraction which is aimed to capture a strong local soundness guarantee.

Local soundness requires that the extracted local witness is consistent with the relation and doesn't lead to a local contradiction, that is, it satisfies the local constraints associated to some set $S$. The no-signaling requirement is defined for any two sets $S, S^{\prime}$ where $S^{\prime} \subseteq S$ and of size at most $K$. It states that the result of programming extraction for $S$ and then output only the extracted value for $S^{\prime}$, should be indistinguishable from the result of programming extraction for $S^{\prime}$ and output the extracted value for $S^{\prime}$. Intuitively, this strengthens locality by requiring that the small parts of the local witness are extracted independently from rest.

We next outline the construction of pairing-based quasi-arguements for two specific languages of interest, satisfiability of linear and quadratic relations on committed values. For ease of presentation we do so for symmetric bilinear groups but we streess out that we also translate these to the more efficient setting of asymetric bilinear groups. We will later rely on these quasi arguments to construct a delegation scheme for polynomial sized arithmetic 
circuits but we emphasize that these constructions are of independent interest; they capture a form of "succinct" aggregation of relations and -importantly- they do so under standard falsifiable assumptions. While full knowledge soundness is not achieved, the weakened notion of no-signaling extraction might be enough for some applicaitons. Thus, we choose to present them in full generality.

\subsubsection{Preliminaries}

In this section we introduce some necessary preliminaries for the construction of the quasi arguments for linear and quadratic relations. First, we introduce the Matrix and Kernel DiffieHellman [EHK+13; MRV16] assumption families. Then we introduce Quasi-Adaptive NIZK [JR13] and sketch the QA-NIZK construction for membership in linear spaces of [KW15] and finally the knowledge transfer arguments introduced in [GR19] which allow to construct QANIZK under falsifiable assumptions in some more restricted setting.

Cryptographic assumptions. We introduce informally the Matrix and Kernel Diffie-Hellman assumptions [EHK+13; MRV16]. These are natural generalizations of assumptions used in group based cryptography (either with pairings or not). Both assumption families are parametrized by distributions over matrices in $\mathbb{Z}_{p}$, that is, we consider distribution ensembles $\mathcal{D}_{\ell, k}$ that output matrices in $\mathbb{Z}_{p}^{\ell \times k}$. When $\ell=k+1$ we simply write $\mathcal{D}_{k}$.

The $\mathcal{D}_{\ell, k}$-Matrix Diffie-Hellman Assumption $\left(\mathcal{D}_{\ell, k}-\mathrm{MDDH}\right)$ states that elements in the image of a matrix $\mathbf{A}$ sampled from $\mathcal{D}_{\ell, k}$ are computationally indistinguishable from uniformly random elements.

Assumption. (Informal) $\mathcal{D}_{\ell, k}-\mathrm{MDDH}$ holds in $\mathbb{G}$ if the distributions $\{[\mathbf{A}],[\mathbf{A} w]\}$ and $\{[\mathbf{A}],[z]\}$ are computationally indisthinguishable, where $w, z$ are random elements of $\mathbb{Z}_{p}^{k}$ and $\mathbb{Z}_{p}^{\ell}$ respectively, and $\mathbf{A} \leftarrow \mathcal{D}_{\ell, k}$.

Consider the uniform distribution $\mathcal{U}_{2,1}$ that outputs random elements in $\mathbb{Z}_{p}^{2 \times 1}$. It is easy to assert that the $\mathcal{U}_{2,1}-\mathrm{MDDH}$ assumption is equivalent to the Decisional Diffie-Hellman assumption in $\mathbb{G}^{8}{ }^{8}$ In the setting of symmetric bilinear groups -where the DDH assumption does not hold- we consider a slightly stronger assumption, namely the Decisional Linear assumption (DLIN) [BBS04]. This assumption can be stated as the $\mathcal{L}_{3,2}-\mathrm{MDDH}$ assumption, where $\mathcal{L}_{3,2}$ is the distribution

$$
\mathcal{L}_{3,2}=\left\{\left(\begin{array}{cc}
a_{1} & 0 \\
0 & a_{2} \\
1 & 1
\end{array}\right) \mid a_{1}, a_{2} \leftarrow \mathbb{Z}_{p}\right\}
$$

The $\mathcal{D}_{\ell, k}$-Kernel Diffie-Hellman Assumption is a natural computational analogue of the $\mathcal{D}_{\ell, k^{-}}$ MDDH for bilinear groups. The assumption states that it is infeasible to find non-trivial elements of the co-kernel of $\mathbf{A} \leftarrow \mathcal{D}_{\ell, k}$ given $[\mathbf{A}]$.

Assumption. (Informal) $\mathcal{D}_{\ell, k}-\mathrm{MDDH}$ holds in $\mathbb{G}$ if it is computationally hard to find a non-zero element $[z] \in \mathbb{G}^{\ell}$ such that $\left[z^{\top} \mathbf{A}\right]_{T}=[0]_{T}$ given $[\mathbf{A}]$, where $\mathbf{A} \leftarrow \mathcal{D}_{\ell, k}$.

Note that the assumption is efficiently falsifiable since we can check the winning condition by employing the pairing operation, that is check if $e\left([z]^{\top},[\mathbf{A}]\right)=[0]_{T}$. This assumption family abstracts and generalizes various computational assumptions in bilinear group, such as the Simultaneous Double Pairing Assumption [AFG+10].

\footnotetext{
${ }^{8}$ In fact, the assumption is weaker since we implicitly assume a uniformly distributed generator of $\mathbb{G}$, which need not be the case for DDH. To show that it is weaker, it is enough to note that one can randomize a DDH instance.
} 
It is well known that $\mathcal{D}_{\ell, k}-\mathrm{MDDH}$ implies $\mathcal{D}_{\ell, k}$-Kernel Diffie-Hellman assumption. Intuitively, this holds since if we can sample an element $r$ in the co-kernel of $\mathbf{A}$, it always holds that $\boldsymbol{r}^{\top} \mathbf{A} \boldsymbol{w}=\mathbf{0}$ while for a uniformly distributed vector $z$, with overwhelming probability $\boldsymbol{r}^{\top} z \neq 0$, which translates to an efficient distinguisher for the two distributions defined by $\mathcal{D}_{\ell, k}-\mathrm{MDDH}$ assumption.

Quasi-Adaptive NIZK for membership in linear spaces. Quasi-Adaptive NIZK (QA-NIZK) ${ }^{9}$ arguments are NIZK arguments where the CRS is allowed to depend on the specific language for which proofs have to be generated [JR13]. We are interested in the specific language of membership in linear spaces. Specifically, given a matrix $\mathbf{M}$ and a description of a group $g k$, we consider the language of vectors of group elements that lie in the image of $\mathbf{M}$, that is,

$$
\mathcal{L}_{g k, \mathbf{M}}=\{[x] \mid \exists w \text { s.t. } x=\mathbf{M} w\}
$$

In the quasi-adaptive case, we allow the common reference string to depend on $g k, \mathbf{M}$ but an adversary can choose the statement $[x]$ adaptively. There are very efficient constructions in this setting. We briefly describe the construction of Kiltz and Wee [KW15]. First we consider the designated verifier case. Let $\mathbf{M}$ be an $\ell \times n$ matrix. The construction is essentially a hash proof system [CS02]. The crs contains the projection $[\mathbf{B}]=\left[\mathbf{M}^{\top} \mathbf{K}\right]$ for a random secret key $\mathbf{K} \in \mathbb{Z}_{p}^{\ell \times k}$. To prove a statement $[x]=[\mathbf{M}] \boldsymbol{w}$, the prover sends $[\pi]=\boldsymbol{w}^{\top}[\mathbf{B}]$ and the verifier asserts that $[\pi]=[x]^{\top} \mathbf{K}$. Now it is easy to see that this simple protocol is complete. Indeed

$$
\pi=w^{\top}[\mathrm{B}]=\boldsymbol{w}^{\top} \mathbf{M}^{\top} \mathbf{K}=x^{\top} \mathbf{K}
$$

For soundness, roughly speaking, the value $x^{\top} \mathbf{K}$ is random for $x$ that does not belong to the image of $\mathbf{M}$ conditioned on $\mathbf{B}$. Thus, a cheating (even unbounded) prover has only negligible probability of producing a verifying proof for elements not in the image of $\mathbf{M}$.

To make the scheme publicly verifiable, groups equipped with a bilinear map are employed. To enable the verifier to perform the test without knowing the secret $\mathbf{K}$, we also add to the crs the value $[\mathbf{C}]=[\mathbf{K A}]$, where $\mathbf{A}$ is a matrix that satisfies some hardness condition. Now, the verifier can test $e([\pi],[\mathbf{A}])=e\left(\left[x^{\top}\right],[\mathbf{C}]\right)$. Note that this corresponds to multiplying the verification equation of the designated verifier case from the right with $\mathbf{A}$. Now, if

(1) the designated verifier relation does not hold, namely, $\pi \neq x^{\top} \mathbf{K}$ and

(2) the proof verifies, namely $\pi \mathbf{A}=x^{\top} \mathbf{K A}$,

then $[\pi]-\left[x^{\top}\right] \mathbf{K}$ is a non-trivial element in the co-kernel of $[\mathbf{A}]$. Thus, the publicly verifiable scheme is sound if we additionally assume that $\mathbf{A}$ is sampled by a distributions $\mathcal{D}$ such that the $\mathcal{D}$-Kernel Diffie-Hellman assumption holds.

Note that if $\mathbf{M}$ spans the entire linear space, then the language is trivial. In this case, only knowledge soundness is a meaningful property. However, we do not whether knowledge soundness of this construction can be proven under falsifiable assumptions or not.

Knowledge Transfer Arguments. To achieve succinct arguments, in principle, one needs to use shrinking commitments. When trying to use such commitments with QA-NIZK such as [KW15], the aforementioned "triviality" problem arises and it seems like one has to resort to non-falsifiable assumptions or the generic group model. Motivated by the problem of constructing delegation schemes under falsifiable assumptions and in order to overcome the above issue, [GR19] relax the knowledge soundness property.

\footnotetext{
${ }^{9}$ In this work we do not need the zero knowledge property so we omit it from the discussion.
} 
When considering delegation using the natural approach of (deterministically) committing to the wires of the circuit, one can observe that full knowledge soundness seems to be an unnecessarily strong requirement. Indeed, given the input $x$ of the circuit, one can compute (or verify) these commitments efficiently by evaluating the circuit. This means intuitively, that we already know how a "correct" opening of the commitments looks like in the soundness security reduction. [GR19] exploits this fact and manages to relax the knowledge soundness requirement by considering statements of the form "if commitment $[c]$ opens to $w$, then commitment $[d]$ opens to $f(\boldsymbol{w})^{\prime \prime}$ for publicly known function $f$. As we shall see later, they show that this notion of soundness is enough to construct delegation for low-depth circuits. They also construct two knowledge transfer arguments for linear and quadratic relations under falsifiable assumptions. More concretely, they consider statements of the form

- "if $[\boldsymbol{c}]$ opens to $\mathbf{M} \boldsymbol{w}$, then $[d]$ opens to $\mathbf{N} w$ for some publicly known $\mathbf{M}, \mathbf{N}$, and

- "if $\left[c_{1}\right]$ opens to $w_{1}$ and $\left[c_{2}\right]$ opens to $w_{2}$, then $[d]$ opens to $w_{1} \circ w_{2}$ where $\circ$ denotes the pairwise product of vectors.

In the soundness definition, the adversary is required to output the valid opening along with the statement proof-pair. We emphasize that this is only part of the soundness definition and in the protocol execution the prover does not have to output the valid opening. Consider for example the first case for linear relations. An adversary wins if it manages to output a statement $[c],[d]$ with an accepting proof and a $w$ such that $[c]=[\mathbf{M}] \boldsymbol{w}$ but $[d] \neq[\mathbf{N}] w$. Such statements essentially give the guarantee that some a priori knowledge about a commitment is "correctly" transferred to another commitment.

For the former construction, namely linear relations, they use the [KW15] construction where they define $\mathbf{M}$ as a two block matrix where the upper part corresponds to $[c]$ and the lower to $[d]$. Now, using [KW15], the prover simply needs to convince the verifier that $\left[\begin{array}{l}c \\ d\end{array}\right]=\left[\begin{array}{l}\mathbf{M} \\ \mathbf{N}\end{array}\right] \boldsymbol{w}$. They show that this construction is knowledge transfer sound if the upper matrix $\mathbf{M}$ is sampled from a distribution $\mathcal{D}$ for which the $\mathcal{D}-\mathrm{MDDH}$ assumption holds.

For proving the quadratic relations, they do a different analysis of standard techniques used for the construction of pairing-based succinct arguments that exploit the properties of the Lagrange basis.

They also modify these constructions to be compatible with the more efficient setting of asymmetric bilinear groups, under the natural modifications of the required assumption for asymmetric groups.

\subsubsection{Oblivious Trapdoor Generation for Quasi-Arguments}

Similar to the case of no-signaling SSB commitments we define a stornger and easier to work with (in our context) notion that implies the no-signaling property of quasi arguments, oblivious trapdoor generation.

We require that there exists an oblivious key generation algorithm that takes as input (1) a $\mathrm{crs}_{S}$ that allows extraction for a set $S$, and (2) the description of a subset $S^{\prime} \subseteq S$, and generates a $\operatorname{crs}_{S^{\prime}}$ for some set $S^{\prime}$ and a trapdoor ${ }^{10}$ for extracting local witnesses associated to the set $S^{\prime}$ obliviously of $S \backslash S^{\prime}$. We emphasize that the oblivious trapdoor generation algorithm knows neither the description of $S$ nor any information about the trapdoor associated with it. We

\footnotetext{
${ }^{10}$ We modify the quasi-argument defintion of [KPY19] to admit a fixed extractor algorithm that takes as input the statement-proof pair of the adversary, and additionally some secret state produced during the crs generation, -the trapdoor- and extracts the local witness.
} 
require that the new crs is statistically close to the $\mathrm{crs}_{S}$ given as input. The fact that this property implies no-signaling commitments is identical to the case of SSB commitments.

\subsubsection{Quasi-Arguments of Membership in a Linear Space}

We define a quasi-argument of knowledge of some vector $[x] \in \mathbb{G}^{\ell}$ belonging to the image of a matrix $[\mathbf{U}] \in \mathbb{G}^{\ell \times n}$, where $x$ is committed using an SSB commitment. Consider a commitment $[c]$ that is statistically binding on the set $S$. We show that there exists a local and no-signaling extractor which, given some $S \subseteq[n]$ of size $t \leq K$, extracts $\left[x_{S}\right] \in \operatorname{Im}\left(\left[\mathbf{U}_{S}\right]\right)$, where $x_{S} \in \mathbb{Z}_{p}^{t}$ is the vector whose entries are $x_{i}$ and $\mathbf{U}_{S} \in \mathbb{Z}_{p}^{t \times n}$ is the matrix whose rows are the rows of $\mathbf{U}$ indexed by $i$, where $i$ ranges over $S$ in some fixed order. A local constraint $\left[x_{S}\right]$ associated with the set $S$ can be interpreted as satisfying two properties:

(1) $\left[x_{S}\right]$ is consistent with the commitment $[c]$, namely the (uniqe) $S$-opening of $[c]$ is $x_{S}$, and

(2) $\left[x_{S}\right]$ is in the image of $\left[\mathbf{U}_{S}\right]$.

We use the Kiltz and Wee argument of membership in linear spaces [KW15] to construct a quasi argument for linear relations. Details follow.

The argument. Our construction is Kiltz and Wee linear membership argument [KW15] for the matrix [GU], where $\mathbf{G}$ is an SSB commitment key with locality parameter $K$. For completeness, we describe the protocol for this specific matrix. We note that we present the scheme with proof size $k+1$ of [KW15], where $k$ is a parameter of the scheme defined by the underlying assumption, but our construction is also sound for the more efficient instantiation of size $k$. In any case, we emphasize that the parameter is a small constant $(k=2)$.

Let's recall the construction for the matrix $\mathbf{M}=\mathbf{G U}$. The crs contains $[\mathbf{B}]=\left[\mathbf{U}^{\top} \mathbf{G}^{\top} \mathbf{K}\right]$ and $[\mathbf{C}]=[\mathbf{K A}]$ for some random hash key $\mathbf{K}$ and $\mathbf{A}$ drawn from some distribution satisfying a kernel assumption. A proof is computed as $[\pi]=\boldsymbol{w}^{\top}[\mathbf{B}]$, and verification is done by checking if $e([\pi],[\mathbf{A}])=e\left(\left[\boldsymbol{c}^{\top}\right],[\mathbf{C}]\right)$.

Local and No-Signaling extraction. Our strategy to prove local soundness is to show that, apart from extracting $\left[x_{S}\right]$ from $[c]$, we are also able to produce a verifying proof $\left[\pi^{\dagger}\right]$ that $\left[x_{S}\right] \in \operatorname{Im}\left(\mathbf{U}_{S}\right)$. More concretely, on input a $\operatorname{crs~crs}_{S}=\left(\left[\mathbf{A}^{\dagger}\right],\left[\mathbf{B}^{\dagger}\right],\left[\mathbf{C}^{\dagger}\right]\right)$ for membership in the linear space of $\mathbf{U}_{S}$, we can construct another crs that is statistically close to the quasi argument crs for $\mathbf{U}$ and, more importantly, we can extract a local opening $\left[\boldsymbol{x}_{S}\right]$ and a proof $\left[\boldsymbol{\pi}^{\dagger}\right]$ satisfying the verification equation for $\mathrm{crs}_{s}$.

We embed the public parameters $\left[\mathbf{A}^{\dagger}\right],\left[\mathbf{B}^{\dagger}\right],\left[\mathbf{C}^{\dagger}\right]$ of the local linear space argument for $\mathbf{U}_{S}$ in the quasi argument parameters. Although the secret hash key $\mathbf{K}^{\dagger}$ of the local linear argument is statistically hidden, we can still pick a random hash key for all the coordinates by picking another secret key and implicitly define the full secret key as some composition of the two keys. Concretely, given the trapdoor $\mathbf{T}_{S}$ for locally opening SSB commitments we implicitly define $\mathbf{K}=\mathbf{T}_{S} \mathbf{K}^{\dagger}+\mathbf{R}$, where $\mathbf{R}$ is the additional key, so that the proofs for $c=\mathbf{G P}\left(\begin{array}{l}x_{S} \\ x_{\bar{S}}\end{array}\right)=\mathbf{G}_{S} x_{S}+\mathbf{G}_{\bar{S}} x_{\bar{S}}$ are of the form $\pi=c^{\top} \mathbf{K}=\left(\mathbf{G}_{S} x_{S}+\mathbf{G}_{\bar{S}} x_{\bar{S}}\right)^{\top}\left(\mathbf{T}_{S} \mathbf{K}^{\dagger}+\mathbf{R}\right)=x_{S}^{\top} \mathbf{K}^{\dagger}+c^{\top} \mathbf{R}$. In this way a proof for the local argument can be retrieved as $\left[\pi^{\dagger}\right]=[\pi]-\left[c^{\top}\right] \mathbf{R}$. This equivalent way of sampling $\mathbf{K}$ allows to compute the crs of the larger linear argument using only $\left[\mathbf{A}^{\dagger}\right],\left[\mathbf{B}^{\dagger}\right],\left[\mathbf{C}^{\dagger}\right]$ and $\mathbf{T}_{S}, \mathbf{R}$. Indeed, we can define $[\mathbf{A}]=\left[\mathbf{A}^{\dagger}\right],[\mathbf{B}]=\left[\mathbf{B}^{\dagger}\right]+\left[\mathbf{U}^{\top} \mathbf{G}^{\top}\right] \mathbf{R}$ and $[\mathbf{C}]=\mathbf{T}_{S}\left[\mathbf{C}^{\dagger}\right]+\mathbf{R}\left[\mathbf{A}^{\dagger}\right]$.

We also show that the crs is indistinguishable for different sets and that there is an oblivious trapdoor generation strategy, and hence we also have a no-signaling extraction strategy. The indistinguishability of the crs follows directly from the indistinguishability of SSB commitment 
keys; it is enough to note that only the commitment key depends on $S$ and all other values can be efficiently computed given only the commitment key ${ }^{11}$. For oblivious trapdoor generation, we use the fact that we can sample an identically distributed commitment key along with a trapdoor -this follows by the oblivious key generation of the commitment scheme- and then we argue in the same way as before: given the commitment key we can sample the rest of crs honestly.

Extension to Knowledge Transfer, Bilateral Spaces and Sum Arguments. We also construct variations of the above protocol, specifically a knowledge transfer version based on [GR19] and two construction suitable for asymmetric bilinear groups.

First we consider the knowledge transfer construction. We first describe the local constraints. Consider two matrices $[\mathbf{M}],[\mathbf{N}]$, and two commitment keys $[\mathbf{G}],[\mathbf{H}]$ statistically binding at $S$. The statement consists of two commitments $[c],[d]$. For the local extraction guarantee w.r.t. set $S$ we require that, given an accepting proof $\pi$ and an opening $w$, we can extract values $\left[x_{S}\right]$, $\left[y_{S}\right]$ such that

(1) $\left[x_{S}\right],\left[y_{S}\right]$ are the unique $S$-openings of $[c]$, [d] w.r.t. commitment keys $\mathbf{G}, \mathbf{H}$ respectively, and

(2) if $\left[x_{S}\right]=\left[\mathbf{M}_{S}\right] \boldsymbol{w}$, then $\left[y_{S}\right]=\left[\mathbf{N}_{S}\right] \boldsymbol{w}$.

The construction and the analysis are identical to the previous case. We simply use the [KW15] construction for the matrix with upper part GM and lower part HN. The only difference in the analysis is on the local extraction case. We argue that we can extract an accepting proof for a crs for the language of linear knowledge transfer for the matrices $\mathbf{M}_{S}, \mathbf{N}_{S}$ and, thus, we also require that the $\mathcal{M}_{S}^{\top}-\mathrm{MDDH}$ assumption holds for every $S$, where $\mathcal{M}_{S}$ is the distribution from which we sample $\mathbf{M}_{S}$.

Finally, we also consider constructions in asymmetric bilinear groups. A variant of the linear subspace QA-NIZK argument given in [GHR15b], and extended to knowledge transfer arguments in [GR19], considers the statement as well as the matrix split between the two groups. We call this argument a linear argument for bilateral spaces. We also consider a particular type of argument for bilateral linear spaces defined in [GHR15b] and called "sum in subspace argument". In this case, the statement is $[x]_{1},[y]_{2}$ and soundness captures that $x+y \in \operatorname{Im}(\mathbf{M}+\mathbf{N})$ given $[\mathbf{M}]_{1},[\mathbf{N}]_{2}$ in the two different source groups. We construct quasi arguments for all these variants with knowledge transfer soundness. Luckily, the constructions as well as the security proofs are minor modifications of the original argument.

\subsubsection{Quasi-Argument of Hadamard Products}

The next quasi arguement construction shows that some vector $c$ is the Hadamard product of two vectors $\boldsymbol{a}, \boldsymbol{b}$, namely $\boldsymbol{c}=\boldsymbol{a} \circ \boldsymbol{b}$. We can naturally define the local constraints here as $c_{S}=\boldsymbol{a}_{S} \circ \boldsymbol{b}_{S}$ for every set $S \subseteq[n]$, where $n$ is the dimension of the vectors. As in the linear case, we care about committed values, that is, the vectors $\boldsymbol{a}, \boldsymbol{b}, \boldsymbol{c}$ are committed and we claim that the openings satisfy the claimed relation.

Our starting point is the "bit-string" argument of [GHR15b]. We observe that it is implicitly a quasi-argument with locality parameter $K=1$ for the set of equations $b_{i}\left(b_{i}-1\right)=0$ for all $i \in[n]$. Next we describe this construction and after that we show it indeed satisfies the

\footnotetext{
${ }^{11}$ Here, we assume the distribution $\mathcal{U}$ that outputs the matrix $[\mathbf{U}]$ is witness samplable, meaning that during sampling, we can also sample the discrete logarithms of [U] which is usually the case. In this work, we only consider such distributions.
} 
no-signaling local soundness property. It will be convenient to directly work with equations of the form $x_{i} y_{i}=z_{i}$ instead of the bit-string argument equations.

The common reference string in [GHR15b] contains what we interpret as three SSB commitment keys $[\mathbf{G}],[\mathbf{H}],[\mathbf{F}]$ with locality parameter $K=1$. It additionally includes the product $[\mathbf{G} \otimes \mathbf{H}]$. The prover gives three commitments $[\boldsymbol{a}],[\boldsymbol{b}],[\boldsymbol{c}]$ w.r.t. $\mathbf{G}, \mathbf{H}, \mathbf{F}$ and claims that the openings satisfy the Hadamard relation. We first note that it is easy to construct an arguement for a related language. Consider the elements $\mathbf{G} \otimes \mathbf{H}$ as a commitment key. The prover can give a commitment to the Kronecker product $z=\boldsymbol{a} \otimes \boldsymbol{b}$ by computing $[t]=[\mathbf{G} \otimes \mathbf{H}] z$. The verifier can then use the pairing to verify the Kronecker product relation, namely it tests that $e([\boldsymbol{c}],[\boldsymbol{d}])=e([\boldsymbol{t}],[1])$ where $[\boldsymbol{c}]=[\mathbf{G}] \boldsymbol{a},[\boldsymbol{d}]=[\mathbf{H}] \boldsymbol{b}$ are commitment to some vectors and are part of the statement. Some simple calculations show that

$$
c d=c \otimes d=\mathrm{G} a \otimes \mathbf{H} b=(\mathrm{G} \otimes \mathbf{H})(a \otimes b)=t
$$

The Kronecker product commitment $t$ is included as part of the proof. Now, from this simple Kronecker product argument, it is easy to prove the Hadamard product. It is enough to note that the Hadamard product is a linear funciton of the Kronecker product, thus, the prover and verifier can use the protocol for linear relations of the previous section.

Local and No-Signaling Extraction. The crucial observation to prove local extraction is that if $\mathbf{G}, \mathbf{H}$ are extractable in one position, say $i, j$ respectively, then $\mathbf{G} \otimes \mathbf{H}$ is extractable at position $n(i-1)+j$. More concretely, lettting $\mathbf{T}_{\mathbf{G}}, \mathbf{T}_{\mathbf{H}}$ be the trapdoors for $\mathbf{G}, \mathbf{H}$ respectively, the trapdoor for the commitment key $\mathbf{G} \otimes \mathbf{H}$ is simply $\mathbf{T}_{\mathbf{G}} \otimes \mathbf{T}_{\mathbf{H}}$. Some straightforward calculations reveal that applying this trapdoor to a commitment with the key $\mathbf{G} \otimes \mathbf{H}$ indeed yields the $n(i-1)+j$-th coordinate of the committed value, which is uniquely defined. In fact, we generalize this for larger locality parameters and we also show that, for some distributions of commitment keys, the no-signaling/oblivious trapdoor generation properties hold if they hold for $\mathbf{G}, \mathbf{H}$.

Consider the simple case of $K=1$ and let all three commitments $\mathbf{G}, \mathbf{H}, \mathbf{F}$ be extractable at the same position $i$. We show that we can extract local openings $\left[x_{i}\right]=\mathbf{T}_{\mathbf{G}}[\boldsymbol{a}],\left[y_{i}\right]=\mathbf{T}_{\mathbf{H}}[\boldsymbol{b}],\left[z_{i}\right]=$ $\mathbf{T}_{\mathbf{F}}[c]$ as well as $\left[w_{i}\right]=\mathbf{T}_{\mathbf{G} \otimes \mathbf{H}}[\boldsymbol{t}]$ such that $z_{i}=x_{i} y_{i}$. Assume for the sake of a contradiction that $z_{i} \neq z_{i}^{\prime}=a_{i} b_{i}$. Since the columns $\boldsymbol{g}_{i}, \boldsymbol{h}_{i}, \boldsymbol{f}_{i}$ are linearly independent from the other columns in $\mathbf{G}, \mathbf{H}, \mathbf{F}$, respectively, if the commitments $[c],[d],[t]$ satisfies $[c] \otimes[d]=e([t],[1])$, then the unique openings at coordinate $i$ satisfy $z_{i}=x_{i} y_{i}$. Now, if $z_{i} \neq z_{i}^{\prime}$, the linear relation does not hold and we can break the underlying QA-NIZK for membership in linear spaces.

For oblivious trapdoor generation, it is enough to note that if the commitment key satisfies this property, so does the above constructions. Indeed, note that using the commitment key, it is enough to produce a crs for membership in subspace language to create the full crs of the protocol.

Extension to Knowledge Transfer Arguments. We extend the quasi-argument local soundness to offer a "knowledge transfer" guarantee. In this case, we essentially commit to commitments. That is, we use an SSB commitment key to commit to multiple commitments and the local openings are commitments themselves. Namely we extract values $\left[x_{i}\right],\left[y_{i}\right],\left[z_{i}\right]$ which are interpreted as commitments w.r.t. some (not necessarily SSB) commitments keys $\mathbf{U}, \mathbf{V}, \mathbf{W}$. We require that no PPT adversary can produce openings $\boldsymbol{a}, \boldsymbol{b}$ such that $x_{i}=\mathbf{U}_{i} \boldsymbol{a}, y_{i}=\mathbf{V}_{i} \boldsymbol{b}$ but $z_{i} \neq \mathbf{W}_{i} \boldsymbol{a} \circ \boldsymbol{b}$. The constraint language for a set $S$ is parametrized by SSB commitments $\mathbf{G}, \mathbf{H}, \mathbf{F}$ binding at $S$ as well as some matrices $\mathbf{U}, \mathbf{V}, \mathbf{W}$. We require that given an accepting proof $\pi$ for a statement $[c],[d],[f]$ and openings $\boldsymbol{a}, \boldsymbol{b}$, we can extract values $\left[x_{S}\right],\left[y_{S}\right],\left[z_{S}\right]$ such that

(1) $\left[x_{S}\right],\left[y_{S}\right],\left[z_{S}\right]$ are the unique $S$-openings of $[c],[d],[f]$ w.r.t. commitment keys $\mathbf{G}, \mathbf{H}, \mathbf{F}$ respectively, and 
(2) if $\left[x_{S}\right]=\left[\mathbf{U}_{S}\right] \boldsymbol{a}$ and $\left[\boldsymbol{y}_{S}\right]=\left[\mathbf{V}_{S}\right] \boldsymbol{b}$, then $\left[z_{S}\right]=\left[\mathbf{W}_{S}\right] \boldsymbol{a} \circ \boldsymbol{b}$.

One might wonder at this point how we commit to commitments which naturally requires multiplication of group elements which is assumed computationally hard. To achieve that, we simply include in the crs the products [GU], [HV], [FW]. Now, we can commit to the $n$ commitments $\mathbf{U}_{i} \boldsymbol{a}$ as $[\mathbf{G U}] \boldsymbol{a}$ and similarly for the other keys.

The knowledge transfer version is essentially the same as in the previous case. The only difference is that we also need to include some additional elements in the crs to allow to the prover to compute the Kronecker product, namely the values $[\mathbf{Q}]=[(\mathbf{G} \otimes \mathbf{H})(\mathbf{U} \otimes \mathbf{V})]$. As in the previous case, we can then exploit the linear relation between the Hadamard product and the Kronecker product. From a correct commitment $[Q](\boldsymbol{a} \otimes \boldsymbol{b})$, we can use the linear knowledge transfer to get a commitment to the Hadamard products w.r.t. the third commitment key, namely $[\mathbf{F W}](\boldsymbol{a} \circ \mathbf{b})$. To show this, we first show that the $\mathcal{G} \otimes \mathcal{H}$-MDDH assumption holds if $\mathcal{G}$-MDDH and $\mathcal{H}$-MDDH hold, where $\mathcal{G}, \mathcal{H}$ are the distributions of $\mathbf{G}, \mathbf{H}$ respectively.

We are also able to extend these techniques to work in asymmetric bilinear groups as well. The construction is somewhat technical, but the core idea is to construct SSB commitments suitable for asymmetric groups, where we "split" the commitments between the two groups, and use the bilateral variants of the linear quasi-arguments discussed in the previous sections.

\subsection{From our Quasi-Arguments to Delegation.}

Using the ideas of [PR17; KPY19], we can derive delegation of computation from quasi arguments for languages encoding the computation. The local constraints capture that each step of the computation was done correctly. First, we present the high level idea for the delegation construction from quasi-arguments. We first show how to delegate low-space TMs/low-width circuits and then we show how to overcome the dependence on space/width.

\subsubsection{Delegating bounded space TM/bounded width circuits}

We first recall the high-level ideas to construct a delegation scheme from quasi arguments of [PR17; KPY19] in the simpler case of bounded space computation. Consider some polynomial time sequential computation which on input $x$ outputs $y$, for example a Turing Machine or an arithmetic circuit. The computation goes through a sequence of states $\mathrm{st}_{0}, \mathrm{st}_{1}, \ldots, \mathrm{st}_{d}$ such that $\mathbf{s t}_{0}$ is consistent with the input, state $\mathrm{st}_{d}$ contains the output $y$, and there's a functional relation between states $\mathrm{st}_{i}, \mathrm{st}_{i+1}$ where $\mathrm{st}_{i+1}=f\left(\mathrm{st}_{i}\right)$ and $f$ is determined by the description of the computation. We first consider the case of bound space computation and discuss later how to remove this constraint. Consider a quasi arguement of locality $K=2|s t|$ where local constraints require that $\mathrm{st}_{i}, \mathrm{st}_{i+1}$ are consistent w.r.t. $f$. The goal is to show that an adversary that makes the quasi-argument verifier accept must (w.o.p) sample $x, y$ such that $y$ is the result of the computation on input $x$.

We can first "program" the local extractor extractor to extract $s_{0}, s t_{1}$, i.e. use locality parameter $K=2|s t|$, where $|s t|$ is a bound on the size of the states (i.e. space of the TM or width of the circuit). Local soundness asserts that state $s_{0}$ is consistent with $x$. Local soundness also implies that $\mathrm{st}_{1}$ is consistent with $\mathrm{st}_{0}$ and hence with $x$ (note that the statement $\mathrm{st}_{1}=f\left(\mathrm{st}_{0}\right)$ depends only on local variables). Now, to show that $\mathrm{st}_{2}$ is also consistent, we jump to another game where first the extractor computes only $\mathrm{st}_{1}$, and in the next game the extractor computes $\mathrm{st}_{1}, \mathrm{st}_{2}$. The crucial observation is that $\mathrm{st}_{1}$ should be still consistent with $x$ in both games. Otherwise, we can distinguish between the common output of extractors for $\mathrm{st}_{0}, \mathrm{st}_{1}$ and $\mathrm{st}_{1}$ or between $\mathrm{st}_{1}$ and $\mathrm{st}_{1}, \mathrm{st}_{2}$, which contradicts the no-signaling property. Importantly, we can efficiently compute the "correct" state $\mathrm{st}_{1}$ since the computation is deterministic, and 
thus the no-signaling distinguisher discribed is indeed efficient. Similarly, consistency of st ${ }_{1}$ and local soundness imply that $\mathrm{st}_{2}$ is also consistent. Now, we can inductively continue until we reach the last state, $\mathrm{st}_{d}$, which corresponds to the output of the computatiaon.

Small width circuit delegation from DLIN. Let $C$ be an arithmetic circuit with width $w$ and depth $d$. We consider the input to correspond to level 0 . Without loss of generality, assume that the circuit has $w$ input and $w$ output wires. In this section we consider the width $w$ to be small, or alternatively, efficiency will depend on $w$.

We follow the circuit arithmetization of [GR19]. The multiplication gates are partitioned in $d$ levels. Each level groups the gates at the same distance from the inputs, without counting linear gates. In this way, the inputs of level $i+1$ are linear combinations of outputs of the $i$ previous levels. We can then express this as constraints describing the computation as

$$
\begin{array}{ll}
\boldsymbol{a}_{i} \circ \boldsymbol{b}_{i}=\boldsymbol{c}_{i} & \text { for } i=1 \text { to } d, \\
\left(\begin{array}{l}
\boldsymbol{a}_{i+1} \\
\boldsymbol{b}_{i+1}
\end{array}\right)=\sum_{0 \leq j \leq i}\left(\begin{array}{l}
\mathbf{D}_{i, j} \\
\mathbf{E}_{i, j}
\end{array}\right) \boldsymbol{c}_{j}=\left(\begin{array}{ll}
\mathbf{D}_{i} & \mathbf{0} \\
\mathbf{E}_{i} & \mathbf{0}
\end{array}\right) \boldsymbol{c} & \text { for } i=0 \text { to } d-1, \\
\boldsymbol{c}_{0}=\boldsymbol{x} \in \mathbb{Z}_{p}^{w} \text { and } \boldsymbol{c}_{d}=\boldsymbol{y} \in \mathbb{Z}_{p}^{w} . &
\end{array}
$$

Vectors $\boldsymbol{a}_{i}, \boldsymbol{b}_{i}, \boldsymbol{c}_{i}$ denote respectively the left, right and output wires of multiplication gates in level $i$. Matrices $\mathbf{D}_{i, j}, \mathbf{E}_{i, j}$ can be naturally derived from the circuit's linear gates. Equation (1) states the relation between output wires and the input wires of a level of multiplication gates.

Now consider a symmetric bilinear group described by $g k$ and consider three SSB commitments $\mathbf{G}, \mathbf{H}, \mathbf{F}$ with locality $K=|w|$ for committing to $w d$-dimensional vectors. We publish in the crs the commitment keys and we we also compute two quasi argument crs:

(1) for membership in linear space crs for the matrix $\left[\mathbf{M}_{1}\right]=\left[\begin{array}{c}\mathbf{F} \\ \mathbf{G D} \\ \mathbf{H E}\end{array}\right]$. Here, $\mathbf{D}, \mathbf{E}$ are the matrices for the linear relations as a whole (note per level). That is, for left and output wires it should hold $\boldsymbol{a}=\mathbf{D} \boldsymbol{c}$, and similarly for right wires.

(2) for hadamard relation for G, H, F. Note that, essentially, this corresponds to yet another quasi argument for membership in linear spaces for $\left[\mathbf{M}_{2}\right]=\left[\begin{array}{c}(\mathbf{G} \otimes \mathbf{H}) \\ \mathbf{F} \Delta\end{array}\right]$ where $\Delta$ captures the linear relation between the Kronecker and Hadamard product, that is $(\boldsymbol{a} \circ \boldsymbol{b})=\Delta(\boldsymbol{a} \otimes \boldsymbol{b})$.

The prover gives the commitments to the left, right, output wires, namely $[L]=[\mathrm{G}] a,[R]=$ $[\mathbf{H}] \boldsymbol{b},[\mathrm{O}]=[\mathbf{F}] \boldsymbol{c}$. Note that these commitments are of size $O(\operatorname{poly}(\kappa) w)$ but independent of $d$. Next, it proves that $[\mathbf{O}],[\mathbf{L}],[\mathbf{R}]$

- lie in the image of $\left[\mathbf{M}_{1}\right]$ using the witness $c$.

- satisfy the Hadamard relations. To do so, it computes a commitment $[\mathbf{Z}]=[(\mathbf{G} \otimes \mathbf{H})](\boldsymbol{a} \otimes \boldsymbol{b})$ and shows using the linear argument that the vector $\left[\left(\begin{array}{l}Z \\ \boldsymbol{O}\end{array}\right)\right]$ lies in the image of $\mathbf{M}_{2}$ using the witness $\boldsymbol{a} \otimes \boldsymbol{b}$.

The verifier checks that (1) the linear proofs verify and (2) that $e([L],[R])=e([Z],[1])$. It also does some additional input/output consistency check which we omit for now and describe next. 
Now, let's see the core of the extraction argument. The inductive claim goes as follows: If we set $[\mathbf{F}]$ extractable for the $i$-th level, namely we the set $S_{i}=\{i w+1, \ldots,(i+1) w\}$, then -conditioned on an accepting proof- extracting the level $i$-th level wires corresponds to the correct values $\left[c_{i}\right]$ w.r.t. the input $c_{0}$. We will handle the base case later when we discuss input/output consistency. For the inductive step, assume the statement is true for $i$. We show that it is true for $i+1$. We proceed as follows:

(1) We first set $\mathbf{G}, \mathbf{H}$ extractable at set $S_{i+1}$ corresponding to the $i+1$-th level in addition to the $\mathbf{F}$ extractable at $S_{i}$. By the no-signaling guarantees the value $\left[c_{i}\right]$ extracted by $[O]$ is still correct.

(2) By the local soundness of the linear quasi argument, the extracted values $\left[\boldsymbol{c}_{i}\right],\left[\boldsymbol{a}_{i+1}\right],\left[\boldsymbol{b}_{i+1}\right]$ must lie in the image of the submatrix of $\mathbf{M}_{1}$ corresponding to these values. This matrix contains the blocks $\mathbf{I}, \mathbf{D}_{i+1}, \mathbf{E}_{i+1}$. Hence the values extracted correspond to the correct values $\left[\boldsymbol{a}_{i+1}\right],\left[\boldsymbol{b}_{i+1}\right]$ w.r.t the input $\boldsymbol{c}_{0}$.

(3) We only set $\mathbf{G}, \mathbf{H}$ extractable at set $S_{i+1}$ and leave $\mathbf{F}$ extractable at the empty set. By the no-signaling guarantees the extracted wires for left and right values $\left[\boldsymbol{a}_{i+1}\right],\left[\mathbf{b}_{i+1}\right]$ are still correct.

(4) In addition to $\mathbf{G}, \mathbf{H}$ extractable at set $S_{i+1}$, we set $\mathbf{F}$ extractable at $S_{i+1}$. Now we argue about local constraint of the Hadamard product. We proceed in two steps:

- By the pairing test $e([\boldsymbol{L}],[\boldsymbol{R}])=e([\boldsymbol{Z}],[1])$ and the assumption that $\left[\boldsymbol{a}_{i+1}\right],\left[\boldsymbol{b}_{i+1}\right]$ are correct we get that

$$
\mathbf{T}_{\mathrm{G}} \boldsymbol{L} \otimes \mathrm{T}_{\mathbf{H}} R=\left(\mathrm{T}_{\mathrm{G}} \otimes \mathrm{T}_{\mathbf{H}}\right)(L \otimes R)=\left(\mathrm{T}_{\mathrm{G}} \otimes \mathrm{T}_{\mathbf{H}}\right) \mathrm{Z}=\mathrm{T}_{\mathrm{G} \otimes \mathrm{H}} \mathbf{Z}
$$

which implies that $z_{i+1}=\boldsymbol{a}_{i+1} \otimes \boldsymbol{b}_{i+1}$. This means that the extracted value of the Kronecker commitment corresponds to the Kronecker product $\boldsymbol{a}_{i+1} \otimes \boldsymbol{b}_{i+1}$ of left and right wires in level $i+1$.

- Working similarly to the step (2), we get that the extracted values $Z_{i+1}, \boldsymbol{O}_{i+1}$ live in the image of $\mathbf{M}_{2}$. It should then be the case that we extract [ $\left.\boldsymbol{c}_{i+1}\right]$ which is the Hadamard product $\boldsymbol{a}_{i+1} \circ \boldsymbol{b}_{i+1}$. This correspond to the correct assignment of output wires in level $i+1$.

(5) Finally, we only set $\mathbf{F}$ extractable at set $S_{i+1}$ and leave $\mathbf{G}, \mathbf{H}$ extractable at the empty set. By the no-signaling guarantees the extracted value $\left[c_{i+1}\right]$ is still correct.

We note that proving this is technically more involved. We need to show that the quasi arguments can be composed well, and they still satisfy the no-signaling properties despite the fact that they share commitment keys. Equivalently one could define and analyze a unified quasi argument to directly work with the circuit "transition funciton". In any case, we omit these details from these technical overview.

Input/Output Consistency. We modify the commitment $\mathbf{F}$ by making it trivially extractable at the input/output levels $0, d$ always, regardless of the extraction set. That is, we "use" the identity matrix $\mathbf{I}_{w}$ for committing to the output wires at the first and last level. This corresponds to augmenting $\mathbf{F}$ with some identity rows. Thus, the verifier can always trivially check the consistency with input/output. Note that the final commitment size grows by $2|w|$, the size of input and output, but these values are part of the statement and don't need to be included in the proof. We stress out the "trivial" identity commitment satisfies the properties needed to be used in our quasi-arguments. 
Assumptions. We next discuss the assumptions we use. For the specific matrices used in the reduction, one can prove soundness of the QA-NIZK argument under falsifiable assumptions since the $S$-submatrices $\mathbf{M}_{1}, \mathbf{M}_{2}$ produce a non-trivial subspace. This means that we rely on the kernel assumption we use for instantiating the QA-NIZK. Noting that MDDH assumptions implies the corresponding kernel assumptions, we can instantiate the quasi argument using the DLIN assumption. Furthermore, the no-signaling property of the commitment keys (the only computational property we use) reduces to an MDDH which we chose on instantiation. Noting that DDH does not hold in symmetric groups we resort to the DLIN assumption which makes the commitments larger by 1 group element. Thus, soundness of the above delegation scheme reduces to the DLIN assumption.

\subsubsection{Overcoming the dependence on space/width.}

The issue with the above construction is that setting $K=O(|s t|)$ yields a proof whose size is linear in the space of the computation. To achieve succinctness in the general case, we need to also perform some "compressing" of the state/width. Kalai et. al. overcome this by considering delegation of RAM computation [KP16] using collision-resistant hash function to compress the width. They use a notion similar to the knowledge transfer notion, namely that no PPT adversary can produce digests $h, h^{\prime}$ and state st such that $h=\operatorname{Hash}(\mathrm{st})$ but $\mathbf{h}^{\prime} \neq \operatorname{Hash}(f(\mathrm{st}))$. Now, a quasi argument for the local constraints $\mathbf{h}_{i}=\operatorname{Hash}\left(f\left(\mathbf{s t}_{i}\right)\right)$ and $\mathrm{h}_{i+1}=\operatorname{Hash}\left(f\left(\mathrm{st}_{i}\right)\right)$ is enough for delegation in the general case.

While previous works achieve this by essentially encoding the computation of generic hash functions in the computation, we use hash functions that are based on Pedersen commitments and have nice algebraic structure and properties. This allows to avoid the concrete cost of encoding arbitrary hash functions in the arithmetic circuit. To this end, we use techniques from [GR19] to derive a structure preserving construction. We present next the basic ideas of their (low depth) delegation construction.

Structure Preserving Delegation for Bounded-Depth Circuits. González and Ràfols [GR19] constructed a delegation scheme with proof-size $O(d \kappa)$ and verification requiring $n$ plus $O(d)$ cryptographic operations, where $n$ is the size of the input, $d$ the depth of the circuit and $\kappa$ a security parameter. Interestingly, the verification procedure of [GR19] can be described completely as a set of pairing product equations. As shown by Abe et al.[AFG+16], cryptographic primitives whose correctness can be stated as equations over bilinear groups are more suited for practically efficient arguments without resorting to generic reductions to a circuit or a $3 \mathrm{CNF}$ formula.

In the heart of the delegation scheme of [GR19] lie the two knowledge transfer arguments for linear and quadratic relations described before. To delegate the computation of an arithmetic circuit, the multiplication gates are partitioned in $d$ levels. Each level groups the gates at the same distance from the inputs, without counting linear gates. In this way, the inputs of level $i+1$ are linear combinations of outputs of the $i$ previous levels. A prover commits to the left, right, and output wires of each level as $L_{i}, R_{i}, O_{i}$. In the first $d$ arguments $f$ is a linear function and the argument handles the linear relations between the input wires (the openings of $L_{i}, R_{i}$ ) of level $i$ and the output wires of all previous levels (the openings of $O_{1}, \ldots, O_{i-1}$ ). In the next $d$ arguments $f$ is the hadamard product so that the opening of $O_{i}$ is the the hadamard product of the openings of $L_{i}$ and $R_{i}$. The fact that the verifier can check the commitment to the first level using the public input and a simple inductive argument over the levels shows that the output must be correct.

More concretely, starting from a correct commitment $O_{0}$ (directly checked for consistency 
with input $x$ from the verifier) we conclude that $L_{1}, R_{1}$ by the knowledge transfer guarantee of the linear argument. Since $L_{1}, R_{1}$ are correct w.r.t. $x, O_{1}$ is also correct w.r.t. $x$ by the knowledge transfer guarantee of the quadratic arguement. We continue this way and we conclude that $O_{d}$ is a correct commitment to the output of the computation. Now, we simply need to check that the claimed output $y$ is a correct opening for that latter commitment.

As for soundness, the quadratic knowledge transfer arguement requires a specific (not uniform) distribution for the commitment keys where each row of the matrix of the commitment key is the result of evaluating Lagrange polynomials at a different random point. Thus, soundness relies on a width-size assumption, namely "R-Rational Strong Diffie Hellman" assumption [GR19] which is proven secure in the Generic Group Model. We stress out that we modify the construction of [GR19] to overcome the need for a $q$-size assumption and rely only on a constnt-size one, albeit at the cost of having a quadratic crs and prover computation.

Succinct Publicly Verifiable Delegation for polynomial size circuits. We use the technique of [GR19] to overcome the width dependency in the above construction. The problem with this construction is that we need to rely on simple soundness of the underlying Kiltz and Wee QA-NIZK. However if we try to "shrink" the per-level information to eliminate the width dependence, the subspaces used become trivial and knowledge soundness seems to be needed.

We overcome this by relying on the knowledge transfer analysis of Kiltz and Wee used in [GR19]. To exploit this to construct delegation, we proceed as follows: we keep the same skeleton of the small-width circuit protocol, but instead of directly committing to the left, right and output wires, we commit to commitments of them. That is, for each level we compute three shrinking commitments -with size independent of the width-corresponding to left, right and output wires for that level, and we commit to these commitments (by including appropriate group elements in the crs). Furthermore, we use the knowledge transfer variants of the quasi arguements.

Now, our no-signaling extractor works as in the small-width case, but instead of the wires for some level, it outputs the commitments for the wires in this level. By the knowledge transfer guarantees, we establish that the extracted values for each level satisfy:

(1) if $O_{i}$ is a commitment to $c_{i}$ then $L_{i+1}$ and $R_{i+1}$ are commitments to $\boldsymbol{a}_{i+1}, \boldsymbol{b}_{i+1}$,

(2) if $L_{i+1}$ and $R_{i+1}$ are commitments to $\boldsymbol{a}_{i+1}$ and $\boldsymbol{b}_{i+1}$ respectively, then $O_{i+1}$ is a commitment to $c_{i+1}$

Extracting these values in a no-signaling way, as in the bounded space case, yields soundness for the delegation scheme. The analysis is almost the same and the only difference is that the knowledge transfer guarantee implies some hardness assumption (MDDH) on the distribution of matrices used as parameters, in this case, the width commitment keys. To satisfy this using constant size assumptions, we use a simple variation of Pedersen commitments where the commitment keys satisfy the DLIN assumption.

Remark (Uniform vs Non-Uniform Computation). Our construction can be used for any nonuniform computation, namely polynomial size arithmetic circuits, while previous works such as [PR17; KPY19] focus on delegating uniform computations: Turing or RAM machines. While this is a stronger result, we achieve it using a long (quadratic in the size/time of computation) crs while the work of [KPY19] achieves a short (i.e. sublinear) crs. One motivation for working directly with poly-size circuits is for practical efficiency: we utilize the rich SNARK toolbox without the need to encode expensive cryptographic operations as arithmetic circuits, namely, we focus on structure preserving constructions. While we have an inefficient (quadratic) prover, in all other aspects we achieve optimal efficiency comparable with SNARGs from non-falsifiable 
assumptions. We believe that this is a promising direction and an interesting open problem is to improve the prover to quasi-linear using these techniques. This would yield a delegation scheme for poly-size circuits that directly competes with the aforementioned non-falsifiable based constructions in all aspects, effectively making the use of non-falsifiable assumptions unjustifiable in the context of deterministic computation. We also leave as future work exploring to what extend our techniques can be applied for delegating uniform computations and if this would give some improvement over existing constructions.

Remark (On bootstrapping and proof composition). To improve efficiency (crs size), [KPY19] use the bootstrapping technique which involves proof composition. Our techniques seem to be incompatible with the bootstrapping technique. This is because the crs of our construction depends on the circuit and we cannot directly reuse a crs for different computations. We leave as future work to examine if we can modify our techniques to be able to apply the bootstrapping technique. We also stress out that this might prove to be an interesting direction for improvements in practical efficiency as well due to some recent results in proof-composition techniques [BCMS20; BCL+21].

\subsection{NIZK, SNARKs and Compact NIZK}

We can use standard techniques to turn our delegation scheme into a NIZK argument. Essentially, the prover needs to prove knowledge of (additional) secret input wires $w$ and proof that $C(x, w)=y$ for some secret input $w$. Given the "structure preserving" properties of our delegation scheme, we can directly apply the Groth Sahai proof system [GS08] ${ }^{12}$ on the set of verification equations. In general, all we need to achieve knowledge soundness is an extractable (and hiding) commitment for extracting the witness $w$. Depending on the properties of the extractable commitment scheme we get different NIZK flavors.

If the commitments to the inputs are succinct, the construction yields a SNARK for NP. Such commitments are widely employed in SNARKs, but their security relies on non-standard assumptions: either knowledge type assumptions such as $q$-Knowledge of Exponents assumption [GGPR13] or the generic group model [Gro16]. If we take for example the zk-SNARK from [DFGK14], the size of $q$ is the number of field elements extracted from a valid proof. Indeed, the proof of soundness requires the extraction of all the circuit wires, which are later used to break some falsifiable $q$-assumption. Consequently, the knowledge assumption is of size $q=O(|C|)$. By reducing the number of extracted values from $O(|C|)$ to $|w|$, we reduce the size of the underlying knowledge assumption to $q=|w|<|C|$.

If we use the "bit-string" argument of [GHR15b] to show knowledge of $\boldsymbol{b} \in\{0,1\}^{n}$, we get extractable commitments of size $n+O(1)$ group elements based on a constant-size falsifiable assumption. Combining this extractable commitment with our delegation scheme yields a NIZK argument for circuit satisfiability with proof size $n+O(1)$ groups elements, or equivalently of size $O(n \kappa)$.

Finally, we can then use the techniques of Katsumata et al. [KNYY19; KNYY20] to construct a compact NIZK. The construction of Katsumata et al. is based on a non-compact NIZK argument for $\mathrm{NC}^{1}$ plus a symmetric key encryption scheme $(\mathrm{K}, \mathrm{E}, \mathrm{D})$ where the size of $\mathrm{E}(K, m)$ is $|m|+$ poly $(\kappa)$. Instead of committing to the input $x$ of a circuit $C$, we need to compute $K \leftarrow \mathrm{K}\left(1^{\kappa}\right)$ to obtain $c t \leftarrow \mathrm{E}(K, x)$ and give a NIZK argument of knowledge of some $K \in\{0,1\}^{\text {poly }(\kappa)}$ such that $C(D(K, c t))=1$. We note that we can straightforward use this idea to construct compact NIZK for any circuit by simply plugging our NIZK argument based on the commitments of

\footnotetext{
${ }^{12}$ This can be also achieved in a more efficient way (concretely) by directly using hiding commitments for the delegation scheme.
} 
[GHR15b]. The final proof is of size $|c t|+|K| \operatorname{poly}(\kappa)+|\pi|=n+\operatorname{poly}(\kappa)$ and is sound for any polynomial size circuit.

\section{Preliminaries}

\subsection{Notation}

For $n \in \mathbb{N}$, let $[n]$ be the set $\{1, \ldots, n\}$. For vectors $\boldsymbol{a}=\left(a_{i}\right)_{i \in[n]}, \boldsymbol{b}=\left(b_{i}\right)_{i \in[n]} \in \mathbb{Z}_{p}^{n}$, we denote $\boldsymbol{a} \circ \boldsymbol{b}=\left(a_{i} b_{i}\right)_{i \in[n]}$ the Hadamard product of them, and for matrices $\mathbf{A}=\left(a_{i, j}\right)_{i \in\left[n_{1}\right], j \in\left[m_{1}\right]} \in \mathbb{Z}_{p}^{n_{1} \times m_{1}}$, $\mathbf{B} \in \mathbb{Z}_{p}^{n_{2} \times m_{2}}$ we denote $\mathbf{A} \otimes \mathbf{B}=\left(a_{i, j} \mathbf{B}\right)_{i \in\left[n_{1}\right], j \in\left[m_{1}\right]} \in \mathbb{Z}_{p}^{n_{1} n_{2} \times m_{1} m_{2}}$ their Kronecker product. We will be using the mixed-product property of kronecker products, which says that $(\mathbf{A} \otimes \mathbf{B})(\mathbf{C} \otimes \mathbf{D})=$ $(\mathbf{A C}) \otimes(\mathbf{B D})$ whenever $\mathbf{A}, \mathbf{B}, \mathbf{C}, \mathbf{D}$ have the appropriate dimensions. When $n_{1}=n_{2}$ we denote by $\mathbf{A} \mid \mathbf{B} \in \mathbb{Z}_{p}^{n_{1} \times m_{1}+m_{2}}$ their vertical concatenation. For $\boldsymbol{x}, \boldsymbol{y} \in \mathbb{Z}_{p}^{n}$ we write $\boldsymbol{x} \leq \boldsymbol{y}$ if and only if $x_{i} \leq y_{i}$ for all $i \in[n]$. We consider vectors of sets $S=\left(S_{1}, \ldots, S_{\ell}\right)$, where $S_{i} \subseteq\left[n_{i}\right]$ for $i \in[\ell]$ and $n_{i} \in \mathbb{N}$, and extend set operations entry-wise. That is $S^{\prime} \subseteq S$ if and only if $S_{i}^{\prime} \subseteq S_{i}$ for all $i \in[\ell]$, and $|S|=\left(\left|S_{1}\right|, \ldots,\left|S_{\ell}\right|\right)$. For $\boldsymbol{n} \in \mathbb{N}^{\ell},[\boldsymbol{n}]=\left(\left[n_{1}\right], \ldots,\left[n_{2}\right]\right)$.

We use implicit group notation. Let $g k=\left(p, \mathbb{G}_{1}, \mathbb{G}_{2}, \mathbb{G}_{T}, e, \mathcal{P}_{1}, \mathcal{P}_{2}\right) \leftarrow \mathcal{G}\left(1^{\kappa}\right)$ be the description of an asymmetric bilinear group of size $p=O\left(2^{\kappa}\right)$ equipped with an efficient bilinear map $e: \mathbb{G}_{1} \times \mathbb{G}_{2} \rightarrow \mathbb{G}_{T}$, where $\mathcal{P}_{\mu}$ is a generator of $\mathbb{G}_{\mu}, \mu \in\{1,2\}$. We assume all our algorithms receive as input $g k$ sampled from $\mathcal{G}\left(1^{\lambda}\right)$, although in some abstract definitions is not necessarily the description of a bilinear group. For $r \in \mathbb{Z}_{p}$ we denote $[r]_{\mu}=r \mathcal{P}_{\mu}$ for $\mu \in\{1,2, T\}$ and $\mathcal{P}_{T}=e\left(\mathcal{P}_{1}, \mathcal{P}_{2}\right)$. For a vector $a \in \mathbb{Z}_{p}^{n}$ and matrix $\mathbf{A} \in \mathbb{Z}_{p}^{n \times m}$ we denote with $[\boldsymbol{a}]_{\mu},[\mathbf{A}]_{\mu}$ the natural embedding of $\boldsymbol{a}$, A in $\mathbb{G}_{\mu}$, respectively.

Sub-vectors and Sub-matrices. Let $S=\left\{s_{1}, \ldots, s_{t}\right\} \subseteq[n]$ and $\bar{S}=\left\{\bar{s}_{1}, \ldots, \bar{s}_{n-t}\right\}$ the set $[n] \backslash S$. We use an algebraic notation for the sub-vector $x_{S}$ and sub-matrix $\mathbf{G}_{S}$ of some $x \in \mathbb{Z}_{p}^{n}$ and $\mathbf{G} \in \mathbb{Z}_{p}^{m \times n}$ respectively. Let $\mathbf{P}_{S} \in\{0,1\}^{n \times n}$ the permutation matrix defining the ordering $s_{1}, \ldots, s_{t}, \bar{s}_{1}, \ldots, \bar{s}_{n-t}$. That is, $\mathbf{P}_{S} \boldsymbol{e}_{s_{i}}=\boldsymbol{e}_{i}$ and $\mathbf{P}_{\bar{S}} \boldsymbol{e}_{\bar{s}_{i}}=\boldsymbol{e}_{i+t}$, where $\boldsymbol{e}_{i}$ is the $i$-th unitary vector of size $n$. We may simply write $\mathbf{P}$ when $n, S$ are clear from the context. We also define the matrix $\Sigma_{S}=\left(\mathbf{I}_{t} \mid \mathbf{0}_{t \times n-t}\right)$. We may omit the subscript when the values are clear from the context.

We denote by $x_{S} \in \mathbb{Z}_{p}^{t}, \mathrm{G}_{S} \in \mathbb{Z}_{p}^{k \times t}$ the sub-vector and sub-matrix containing the elements or columns with indices in $S \subseteq[n]$ of $x \in \mathbb{Z}_{p}^{n}$ and $\mathbf{G} \in \mathbb{Z}_{p}^{k \times n}$, respectively.

Fact 1. For any $x \in \mathbb{Z}_{p}^{n}$ and any $S^{\prime} \subseteq S \subseteq[n]$ it holds that:

i. $\mathbf{P}_{S} \boldsymbol{x}=\left(\begin{array}{c}x_{S} \\ x_{\bar{S}}\end{array}\right)$ and $\mathbf{G} \mathbf{P}_{S}^{\top}=\left(\mathbf{G}_{S} \mid \mathbf{G}_{\bar{S}}\right)$.

ii. $x_{S}=\Sigma_{S} \mathbf{P}_{S} x$ and $\mathbf{G}_{S}=\mathbf{G P}_{S}^{\top} \Sigma_{S}^{\top}$.

iii. $\mathrm{G} x=\mathrm{G}_{S} x_{S}+\mathrm{G}_{\bar{S}} x_{\bar{S}}$.

iv. Let $x_{S^{\prime} \mid S}=\Sigma_{S \mid S^{\prime}} \mathbf{P}_{S^{\prime} \mid S} \boldsymbol{x}_{S}$, where $\mathbf{P}_{S^{\prime} \mid S}$ is some permutation matrix such that $\mathbf{P}_{S^{\prime} \mid S} \boldsymbol{x}_{S}=\left(\begin{array}{c}\boldsymbol{x}_{S^{\prime}} \\ x_{S \mid S^{\prime}}\end{array}\right)$ and $\boldsymbol{\Sigma}_{S^{\prime} \mid S}=\left(\mathbf{I}_{\left|S^{\prime}\right|} \mid \mathbf{0}_{\left|S^{\prime}\right| \times t-\left|S^{\prime}\right|}\right) \cdot \boldsymbol{x}_{S^{\prime} \mid S}=x_{S^{\prime}}$ and $\mathbf{G}_{S^{\prime} \mid S}=\mathbf{G}_{S^{\prime}}$.

When $x=\mathbf{U} w$, for some matrix $\mathbf{U} \in \mathbb{Z}_{p}^{n \times m}$ and $\boldsymbol{w} \in m$, we abuse of notation and also write $\mathbf{U}_{S}$ for $\boldsymbol{\Sigma}_{S} \mathbf{P}_{S} \mathbf{U}$ so that $\boldsymbol{x}_{S}=\mathbf{U}_{S} \boldsymbol{w}$.

We extend this notation to two sets $S_{1} \subseteq\left[n_{1}\right], S_{2} \subseteq\left[n_{2}\right]$ and for $x \in \mathbb{Z}_{p}^{n_{1} n_{2}}$ define $x_{S_{1}, S_{2}} \in$ $\mathbb{Z}_{p}^{\left|S_{1}\right| \cdot\left|S_{2}\right|}$ as $x_{S_{1}, S_{2}}=\left(\boldsymbol{x}_{(i-1) n_{2}+j}: i \in S_{1}\right.$ and $\left.j \in S_{2}\right)$ in some fixed order. For matrices instead we define $\mathbf{G}_{S_{1}, S_{2}}=\left(q_{\ell,(i-1) n_{2}+j}: \ell \in[k], i \in S_{1}\right.$ and $\left.j \in S_{2}\right) \in \mathbb{Z}_{p}^{k \times\left|S_{1}\right| \cdot\left|S_{2}\right|}$, where $k$ is the number of columns of $\mathbf{G}$. Similarly as before, the following holds. 
Fact 2. For any $x \in \mathbb{Z}_{p}^{n_{1} n_{2}}$ and any $S_{1}^{\prime} \subseteq S_{1} \subseteq\left[n_{1}\right], S_{2}^{\prime} \subseteq S_{2} \subseteq\left[n_{2}\right]$ it holds that:

i. For some permutation matrix $\Pi \in \mathbb{Z}_{p}^{n_{1} n_{2} \times n_{1} n_{2}},\left(\mathbf{P}_{S_{1}} \otimes \mathbf{P}_{S_{2}}\right) x=\Pi\left(\begin{array}{l}x_{S_{1}, S_{2}} \\ x_{S_{1}, \bar{s}_{2}} \\ x_{\bar{S}_{1}, S_{2}} \\ x_{\bar{s}_{1}, \bar{s}_{2}}\end{array}\right)$ and $\mathbf{G}\left(\mathbf{P}_{S_{1}}^{\top} \otimes \mathbf{P}_{S_{2}}^{\top}\right)=$ $\left(\mathbf{G}_{S_{1}, S_{2}}\left|\mathbf{G}_{S_{1}, \bar{S}_{2}}\right| \mathbf{G}_{\bar{S}_{1}, S_{2}} \mid \mathbf{G}_{\bar{S}_{1}, \bar{S}_{2}}\right) \Pi^{\top}$.

ii. $x_{S_{1}, S_{2}}=\left(\Sigma_{S_{1}} \otimes \Sigma_{S_{2}}\right)\left(\mathbf{P}_{S_{1}} \otimes \mathbf{P}_{S_{2}}\right) x$ and $\mathbf{G}_{S_{1}, S_{2}}=\mathbf{G}\left(\mathbf{P}_{S_{1}}^{\top} \otimes \mathbf{P}_{S_{2}}^{\top}\right)\left(\Sigma_{S_{1}}^{\top} \otimes \Sigma_{S_{2}}^{\top}\right)$.

iii. $\mathrm{G} x=\mathrm{G}_{S_{1}, S_{2}} x_{S_{1}, S_{2}}+\mathrm{G}_{S_{1}, \bar{S}_{2}} x_{S_{1}, \bar{S}_{2}}+\mathrm{G}_{\bar{S}_{1}, S_{2}} x_{\bar{S}_{1}, S_{2}}+\mathrm{G}_{\bar{S}_{1}, \bar{S}_{2}} x_{\bar{S}_{1}, \bar{S}_{2}}$.

iv. Let $\boldsymbol{x}_{S_{1}^{\prime}, S_{2}^{\prime} \mid S_{1}, S_{2}}=\left(\boldsymbol{\Sigma}_{S_{1}^{\prime} \mid S_{1}}^{\top} \otimes \boldsymbol{\Sigma}_{S_{2}^{\prime} \mid S_{2}}^{\top}\right)\left(\mathbf{P}_{S_{1}^{\prime} \mid S_{1}} \otimes \mathbf{P}_{S_{2}^{\prime} \mid S_{2}}\right) \boldsymbol{x}_{S_{1}, S_{2}}$ and $\mathbf{G}_{S_{1}^{\prime}, S_{2}^{\prime} \mid S_{1}, S_{2}}=\mathbf{G}\left(\mathbf{P}_{S_{1}^{\prime} \mid S_{1}}^{\top} \otimes \mathbf{P}_{S_{2}^{\prime} \mid S_{2}}^{\top}\right)\left(\boldsymbol{\Sigma}_{S_{1}^{\prime} \mid S_{1}}^{\top} \otimes\right.$ $\boldsymbol{\Sigma}_{S_{2}^{\prime} \mid S_{2}}^{\top}$. Then $x_{S_{1}^{\prime}, S_{2}^{\prime} \mid S_{1}, S_{2}}=\boldsymbol{x}_{S_{1}^{\prime}, S_{2}^{\prime}}$ and $\mathbf{G}_{S_{1}^{\prime}, S_{2}^{\prime} \mid S_{1}, S_{2}}=\mathbf{G}_{S_{1}^{\prime}, S_{2}^{\prime}}$

\subsection{Cryptographic Assumptions}

Definition 1. Let $k, \ell \in \mathbb{N}$. We call $\mathcal{D}_{\ell, k}$ (resp. $\mathcal{D}_{k}$ ) a matrix distribution if it outputs in PPT time, with overwhelming probability matrices in $\mathbb{Z}_{p}^{\ell \times k}$ (resp. in $\mathbb{Z}_{p}^{(k+1) \times k}$ ). For a matrix distribution $\mathcal{D}_{k}$, we denote as $\overline{\mathcal{D}}_{k}$ the distribution of the first $k$ rows of the matrices sampled according to $\mathcal{D}_{k}$.

Assumption 1. Let $\mathcal{D}_{\ell, k}$ be a matrix distribution. For all non-uniform PPT adversaries $\mathcal{A}$ and relative to $g k \leftarrow \mathcal{G}\left(1^{\kappa}\right), \mathrm{A} \leftarrow \mathcal{D}_{\ell, k}$ and the coin tosses of adversary $\mathcal{A}$,

1. the Kernel Matrix Diffie-Hellman Assumption holds in $\mathbb{G}_{\gamma}[$ MRV16] if

$$
\operatorname{Pr}\left[[r]_{3-\gamma} \leftarrow \mathcal{A}\left(g k,[\mathbf{A}]_{\gamma}\right): r^{\top} \mathbf{A}=0\right]=\operatorname{negl}(\kappa),
$$

2. the Split Kernel Matrix Diffie-Hellman Assumption [GHR15b] holds if

$$
\operatorname{Pr}\left[[\boldsymbol{r}]_{1},[s]_{2} \leftarrow \mathcal{A}\left(g k,[\mathbf{A}]_{1},[\mathbf{A}]_{2}\right): r \neq s \wedge r^{\top} \mathbf{A}=s^{\top} \mathbf{A}\right]=\operatorname{negl}(\kappa) .
$$

Assumption 2. Let $\mathcal{D}_{\ell, k}$ be a matrix distribution and $g k \leftarrow \mathcal{G}\left(1^{\kappa}\right)$. For all non-uniform PPT adversaries $\mathcal{A}$ and relative to $g k \leftarrow \mathcal{G}\left(1^{\kappa}\right), \mathbf{A} \leftarrow \mathcal{D}_{\ell, k}, \boldsymbol{w} \leftarrow \mathbb{Z}_{p}^{k},[z]_{\gamma} \leftarrow \mathbb{G}_{\gamma}^{\ell}$ and the coin tosses of adversary $\mathcal{A}$,

1. the Matrix Decisional Diffie-Hellman Assumption in $\mathbb{G}_{\gamma}\left(\mathcal{D}_{k}-\mathrm{MDDH}_{\gamma}\right)$ holds if

$$
\left|\operatorname{Pr}\left[\mathcal{A}\left(g k,[\mathbf{A}]_{\gamma},[\mathbf{A} \boldsymbol{w}]_{\gamma}\right)=1\right]-\operatorname{Pr}\left[\mathcal{A}\left(g k,[\mathbf{A}]_{\gamma},[z]_{\gamma}\right)=1\right]\right| \leq \operatorname{negl}(\kappa),
$$

2. the Split Matrix Decisional Diffie-Hellman Assumption in $\mathbb{G}_{\gamma}\left(\mathcal{D}_{k}-\mathrm{SMDDH}_{\gamma}\right)$ holds if

$$
\left|\operatorname{Pr}\left[\mathcal{A}\left(g k,[\mathbf{A}]_{1,2},[\mathbf{A} w]_{\gamma}\right)=1\right]-\operatorname{Pr}\left[\mathcal{A}\left(g k,[\mathbf{A}]_{1,2},[z]_{\gamma}\right)=1\right]\right| \leq \operatorname{negl}(\kappa) .
$$

Assumption 3. Let $\left(\mathcal{D}_{\ell, k}^{1}, \mathcal{D}_{\ell, k}^{2}\right)$ be (possibly correlated) matrix distributions and $g k \leftarrow \mathcal{G}\left(1^{\kappa}\right)$. For all non-uniform PPT adversaries $\mathcal{A}$ and relative to $g k \leftarrow \mathcal{G}\left(1^{\kappa}\right),(\mathbf{A}, \mathbf{B}) \leftarrow\left(\mathcal{D}_{\ell, k}^{1}, \mathcal{D}_{\ell, k}^{2}\right), \boldsymbol{w} \leftarrow$ $\mathbb{Z}_{p}^{k},[z]_{\gamma} \leftarrow \mathbb{G}_{\gamma}^{\ell}$ and the coin tosses of adversary $\mathcal{A}$, the $\left(\mathcal{D}_{\ell, k}^{1}, \mathcal{D}_{\ell, k}^{2}\right)$-Split Matrix Decisional Diffie-Hellman Assumption $\left(\left(\mathcal{D}_{\ell, k}^{1}, \mathcal{D}_{\ell, k}^{2}\right)-\mathrm{MDDH}_{\gamma}\right)$ holds if

$$
\left|\operatorname{Pr}\left[\mathcal{A}\left(g k,[\mathbf{A}]_{1},[\mathbf{B}]_{2},[\mathbf{A} \boldsymbol{w}]_{1},[\mathbf{B} w]_{2}\right)=1\right]-\operatorname{Pr}\left[\mathcal{A}\left(g k,[\mathbf{A}]_{1},[\mathbf{B}]_{2},[s]_{1},[\boldsymbol{t}]_{2}\right)=1\right]\right| \leq \operatorname{negl}(\kappa) .
$$

We also consider stronger versions of these definitions, denoted $\left(\mathcal{D}_{\ell, k}, h\right)-\mathrm{MDDH},\left(\mathcal{D}_{\ell, k}, h\right)$ $\operatorname{SMDDH},\left(\mathcal{D}_{\ell, k}^{1}, \mathcal{D}_{\ell, k}^{2}, h\right)-\mathrm{MDDH}$, where the adversary is also given $h(\mathbf{A})(h(\mathbf{A}, \mathbf{B})$ in the latter $)$ for some (possibly probabilistic) function $h$. 


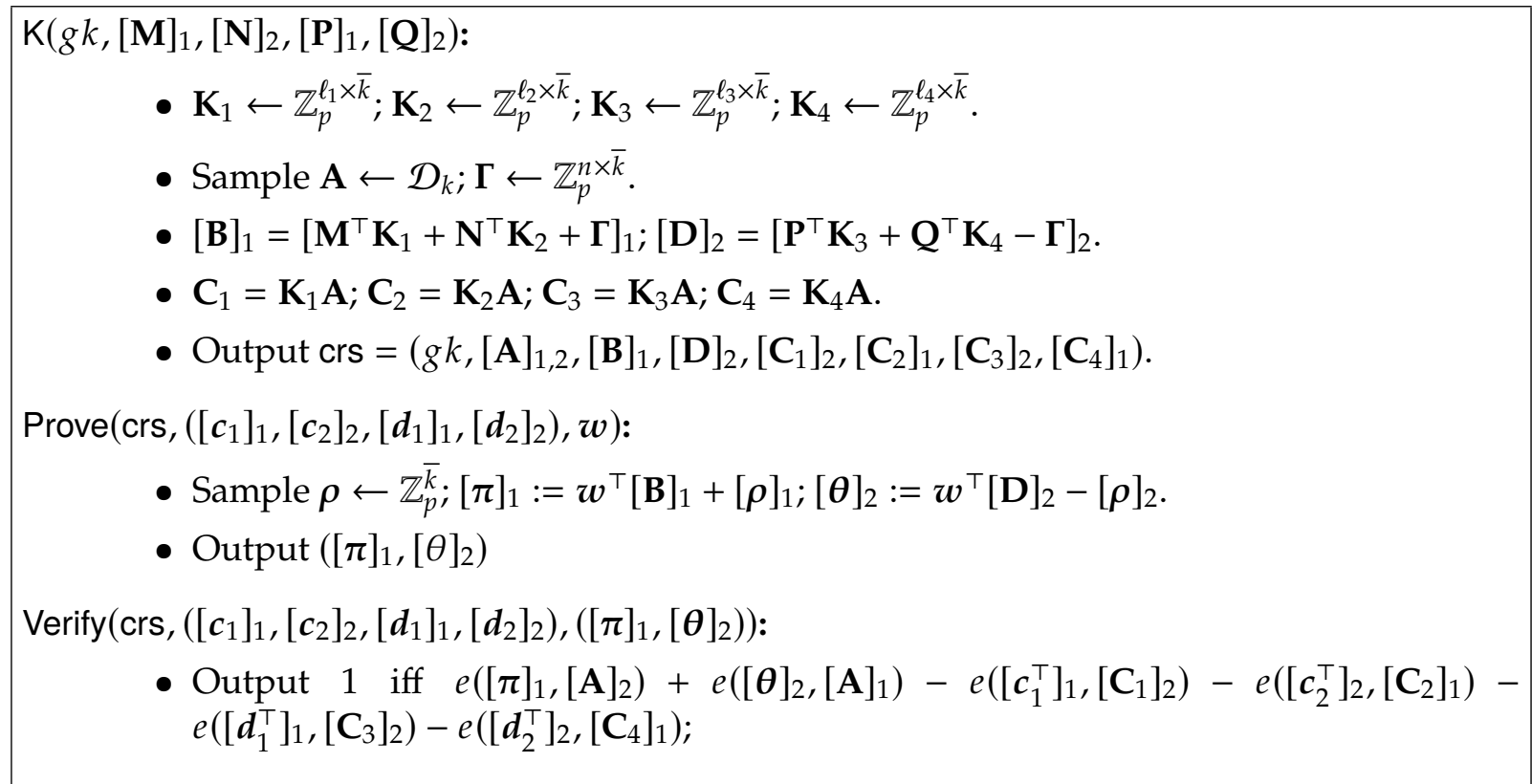

Figure 1: Construction $\Pi_{\text {kt-lin }}$ for $\mathcal{L}_{\text {lin }}^{\text {yes }}, \mathcal{L}_{\text {lin }}^{\text {no }}$. For $\ell_{1}=\ell_{2}$, construction $\Pi_{\text {kt-sum }}$ for $\mathcal{L}_{\text {sum }}^{\text {yes }}, \mathcal{L}_{\text {sum }}^{\text {no }}$ is identical with the only difference that $\mathbf{K}_{2}=\mathbf{K}_{1}$.

\subsection{Arguments of Knowledge Transfer}

In this section we recall arguments of knowledge transfer for membership in linear spaces as defined in [GR19] which in turn is just an instantiation of [KW15]. We also slightly modify the construction to turn it into an argument of knowledge transfer for the sum language, which we will use in later constructions.

Let $g k$ be a bilinear group of order $p$ and $\mathcal{M}, \mathcal{N}, \mathcal{P}, Q$ be matrix distributions outputting matrices $[\mathbf{M}]_{1} \in \mathbb{G}_{1}^{l_{1} \times n},[\mathbf{N}]_{2} \in \mathbb{G}_{2}^{l_{2} \times n}[\mathbf{P}]_{1} \in \mathbb{G}_{1}^{l_{3} \times n}[\mathbf{Q}]_{2} \in \mathbb{G}_{2}^{\ell_{4} \times n}$ respectively. In Fig. 1, we present two arguments of knowledge transfer for (1) the linear membership language

$$
\begin{aligned}
& \mathcal{L}_{\text {lin }}^{\text {yes }}=\left\{\left(\left[c_{1}\right]_{1},\left[c_{2}\right]_{2},\left[d_{1}\right]_{1},\left[d_{2}\right]_{2}\right) \mid \exists w \text { s.t }\left(\begin{array}{c}
c_{1} \\
\boldsymbol{c}_{2}
\end{array}\right)=\left(\begin{array}{c}
\mathbf{M} \\
\mathbf{N}
\end{array}\right) \boldsymbol{w} \text { and }\left(\begin{array}{l}
d_{1} \\
d_{2}
\end{array}\right)=\left(\begin{array}{l}
\mathbf{P} \\
\mathbf{Q}
\end{array}\right) \boldsymbol{w}\right\} \\
& \mathcal{L}_{\text {lin }}^{\text {no }}=\left\{\left(\left[\boldsymbol{c}_{1}\right]_{1},\left[c_{2}\right]_{2},\left[d_{1}\right]_{1},\left[d_{2}\right]_{2}, \boldsymbol{w}\right) \mid\left(\begin{array}{c}
c_{1} \\
c_{2}
\end{array}\right)=\left(\begin{array}{c}
\mathbf{M} \\
\mathbf{N}
\end{array}\right) \boldsymbol{w} \text { and }\left(\begin{array}{l}
d_{1} \\
d_{2}
\end{array}\right) \neq\left(\begin{array}{l}
\mathbf{P} \\
\mathbf{Q}
\end{array}\right) \boldsymbol{w}\right\}
\end{aligned}
$$

and (2) the sum knowledge transfer language

$$
\begin{aligned}
& \mathcal{L}_{\text {sum }}^{\text {yes }}=\left\{\left(\left[c_{1}\right]_{1},\left[c_{2}\right]_{2},\left[d_{1}\right]_{1},\left[d_{2}\right]_{2}\right) \mid \exists w \text { s.t } c_{1}+c_{2}=(\mathbf{M}+\mathbf{N}) \boldsymbol{w} \text { and }\left(\begin{array}{l}
d_{1} \\
d_{2}
\end{array}\right)=\left(\begin{array}{l}
\mathbf{P} \\
\mathbf{Q}
\end{array}\right) \boldsymbol{w}\right\} \\
& \mathcal{L}_{\text {sum }}^{\text {no }}=\left\{\left(\left[c_{1}\right]_{1},\left[c_{2}\right]_{2},\left[d_{1}\right]_{1},\left[d_{2}\right]_{2}, w\right) \mid c_{1}+c_{2}=(\mathbf{M}+\mathbf{N}) \boldsymbol{w} \text { and }\left(\begin{array}{l}
d_{1} \\
d_{2}
\end{array}\right) \neq\left(\begin{array}{l}
\mathbf{P} \\
\mathbf{Q}
\end{array}\right) \boldsymbol{w}\right\} .
\end{aligned}
$$

A knowledge transfer argument is just an argument for the promise problem defined by $\mathcal{L}^{\text {yes }}$ and $\mathcal{L}^{\text {no }}$. Completeness means that an honest proof is accepting for any statement in $\mathcal{L}^{\text {yes }}$. Soundness that any proof for a statement in $\mathcal{L}^{\text {no }}$, which comes with an "advice" $w$, is accepting only with negligible probability.

We use this construction with (1) $\mathbf{Q}=\mathbf{0}$ for the case of linear knowledge transfer and (2) $\mathbf{N}=\mathbf{0}$ for the case of sum knowledge transfer so we prove only these two cases. We stress out that the proofs are easily extended to accommodate for the more general cases. We also strengthen the security requirements by allowing the adversary to get some extra information 
about the language parameters through some (possibly probabilistic) function $h$. We call this property $h$-strong soundness.

For the case of $\Pi_{\mathrm{kt}-\mathrm{lin}}$, when setting $\mathbf{Q}=\mathbf{0}$, the security is shown in [GR19]. The only modification is that we allow the adversary $\mathcal{A}$ to get the discrete logarithms $\mathbf{N}, \mathbf{P}$ and the $h$ information of the MDDH challenge, which does not affect the result of [GR19]. We extend the results of [GR19] to the sum argument. The security proof is essentially identical to the one for the bilateral case of [GR19]. For completeness we give the full proof in Appendix A.

\section{No-Signaling Somewhere Statistically Binding Commitments}

In this section we recall Somewhere Statistically Binding (SSB) commitments and then define two additional notions for SSB commitments: no-signaling extraction and oblivious key generation. The former is a natural adaptation of the definitions of no-signaling extractors from previous works [PR17; KPY19]. We show that the latter implies the former, and we give an efficient instantiation based on any $\mathcal{D}_{k}-\mathrm{MDDH}$ assumption. Finally, we consider the kronecker product of two of these commitments.

We now define somewhere Statistically Binding (SSB) commitment schemes [HW15; FLPS20]. An SSB commitment scheme, as the name suggests, is statistically binding only w.r.t. some variables which are determined during key generation. The commitment key computationally hides any information about this set, meaning that for all "modes" the commitment keys are computationally indistinguishable. Furthermore, the KeyGen outputs a trapdoor which allows to extract (a function of) the values in this set.

It will be useful to consider SSB commitments where committed vectors live in $\mathcal{M}^{n_{1} n_{2}}$ and can be indexed by $i_{1} \in\left[n_{1}\right], i_{2} \in\left[n_{2}\right]$. We consider also 2 locality parameters $K=\left(K_{1}, K_{2}\right)$ with $K_{i} \leq n_{i}$, and extraction sets are of the form $S=\left(S_{1}, S_{2}\right)$ where $S_{i} \subseteq\left[n_{i}\right]$ and $\left|S_{i}\right| \leq K_{i}$, for $i \in\{1,2\}$. We put forward a stronger variant of the index set hiding property, where the distinguisher is also given $h(s k)$ for some function $h$. In this case we will say the SSB commitment is $h$-strong ISH.

Definition 2. Let [·] : $\mathcal{M} \rightarrow G$ be a function, where $\mathcal{M}$ is the message space and $G$ some set. Syntactically, a Somewhere Statistically Binding Commitment Scheme CS is a tuple of algorithms CS = (KeyGen, Com, Extract)

- $(c k, s k) \leftarrow \operatorname{KeyGen}(g k, n, K, S)$ : KeyGen takes as input the parameters $g k, n \in \mathbb{N}^{\ell}$, locality parameters $K \in[n]$ and the sets $S \subseteq[n],|S| \leq K$. It outputs a commitment key $c k$, which may also contain some auxiliary information aux, a secret key $s k$, containing a trapdoor $\tau$ and possibly the random coins used by KeyGen.

- $c \leftarrow \operatorname{Com}(c k, x)$ : Com takes as input the commitment key $c k$ and a vector $x \in \mathcal{M}^{n_{1} \cdot n_{2}}$ and outputs a commitment $c$,

- $y \leftarrow \operatorname{Extract}(\tau, c)$ : Extract takes as input the trapdoor $\tau$ and a commitment $c$, and outputs the value $y \in G$ allegedly equaling $\left[x_{S}\right]$, where $x$ is a valid opening for $c$.

For all $\kappa \in \mathbb{N}, n \in \mathbb{N}^{2}, K \in[n], S_{0}, S_{1} \subseteq[n]$ with $\left|S_{0}\right|,\left|S_{1}\right| \leq K$, CS must satisfy the following properties:

- $h$-Strong Index Set Hiding: for all PPT $\mathcal{D}$

$$
\operatorname{Pr}_{g k \leftarrow \mathcal{G}\left(1^{\kappa}\right)}\left[\mathcal{D}(c k, h(s k))=b \mid \begin{array}{r}
b \leftarrow\{0,1\} \\
(c k, s k) \leftarrow \operatorname{KeyGen}\left(g k, n, K, S_{b}\right)
\end{array}\right] \leq \frac{1}{2}+\operatorname{negl}(\kappa) .
$$


- Somewhere Statistically Binding: for all all, even unbounded $\mathcal{A}$,

$$
\operatorname{Pr}_{g k \leftarrow \mathcal{G}\left(1^{\kappa}\right)}\left[\begin{array}{c|r}
\operatorname{Com}(c k, x)=\operatorname{Com}\left(c k, x^{\prime}\right) & (c k, s k) \leftarrow \operatorname{KeyGen}(g k, n, K, S) ; \\
\text { and } x_{S} \neq x_{S}^{\prime} & \left(x, x^{\prime}\right) \leftarrow \mathcal{A}(c k) ;
\end{array}\right] \leq \operatorname{negl}(\kappa) .
$$

- G-Extractability: for all, even unbounded $\mathcal{A}$

$$
\operatorname{Pr}_{g k \leftarrow \mathcal{G}\left(1^{\kappa}\right)}\left[\begin{array}{c|c}
\exists x \text { s.t. } c=\operatorname{Com}(c k, x) & (c k, s k) \leftarrow \operatorname{KeyGen}(g k, n, K, S) ; c \leftarrow \mathcal{A}(c k) ; \\
\text { and } y \neq\left[x_{S}\right] & y \leftarrow \operatorname{Extract}(\tau, c), \text { where } s k=(\tau, r) ;
\end{array}\right] \leq \operatorname{negl}(\kappa)
$$

Note that an SSB commitment is also "everywhere" computationally binding. This is the case since a breach in binding, namely the ability to produce $c$ that opens to both $x \neq x^{\prime}$, implies the ability to distinguish where the commitment is not statistically binding contradicting the index set hiding property.

We next present an extra property for an SSB commitment scheme which we call $h$-strong no-signaling extraction and is a natural adaptation of the definitions in [PR17; KPY19].

Definition 3. We say the extractor of an SSB commitment scheme CS = (Setup, KeyGen, Com, Extract) with commitment space $C^{13}$ is $h$-strong no-signaling if for any $S^{\prime} \subseteq S \subseteq[n]$, where $|S| \leq K$, and any PPT adversary $\mathcal{D}=\left(\mathcal{D}_{1}, \mathcal{D}_{2}\right)$,

$$
\begin{aligned}
& \operatorname{Pr}_{g k \leftarrow \mathcal{G}\left(1^{\kappa}\right)}\left[\begin{array}{l|l}
\left.\mathcal{D}_{2}\left(c k_{S^{\prime}}, h\left(s k_{S^{\prime}}\right)\right), c, y^{\prime}\right)=1 \mid \begin{array}{r}
\left(c k_{S^{\prime}}, s k_{S^{\prime}}\right) \leftarrow \operatorname{KeyGen}\left(g k, n, K, S^{\prime}\right) \\
c \leftarrow \mathcal{D}_{1}\left(c k_{S^{\prime}}, h\left(s k_{S^{\prime}}\right)\right) ; \text { if } c \notin C: c \leftarrow \perp \\
y^{\prime} \leftarrow \operatorname{Extract}(\tau, c) \text {, where } s k_{S^{\prime}}=(\tau, r) .
\end{array}
\end{array}\right]- \\
& \left.\operatorname{Pr}_{g k \leftarrow \mathcal{G}\left(1^{\kappa}\right)}\left[\mathcal{D}_{2}\left(c k_{S}, h\left(s k_{S}\right)\right), c, y_{S^{\prime}}\right)=1 \mid \begin{array}{r}
\left(c k_{S}, s k_{S}\right) \leftarrow \operatorname{KeyGen}(g k, n, K, S) \\
c \leftarrow \mathcal{D}_{1}\left(c k_{S}, h\left(s k_{S}\right)\right) ; \text { if } c \notin C: c \leftarrow \perp \\
y \leftarrow \operatorname{Extract}(\tau, c) \text {, where } s k=(\tau, r) .
\end{array}\right] \mid \leq \operatorname{negl}(\kappa) .
\end{aligned}
$$

We define also oblivious trapdoor generation. This property states that there exists an oblivious key generation algorithm, that takes a commitment key $c k$ that allows extraction in $S$ and a set $S^{\prime} \subseteq S$, and can produce a fresh commitment key $c k^{\prime}$ and a trapdoor to extract $S^{\prime}$. The distribution of the new key $c k^{\prime}$ is statistically close to that of $c k$ and - importantly - the oblivious key generation algorithm does not get as input the original extraction set $S$. In other words, given a commitment key $c k$ that we know allows extraction for some superset of $S$, we can create a new key with a trapdoor for $S^{\prime}$ without skewing the distribution of $c k$.

Definition 4. An SSB commitment scheme has oblivious trapdoor generation if there exists a PPT algorithm OblKeyGen such that for all $\kappa \in \mathbb{N}, n \in \mathbb{N}^{2}, K \in[n], S \subseteq[n]$, with $|S| \leq K$, and any $S^{\prime}$ such that $S^{\prime} \subseteq S$, and for all, even unbounded $\mathcal{D}=\left(\mathcal{D}_{1}, \mathcal{D}_{2}\right)$,

$$
\begin{aligned}
& \operatorname{Pr}_{g k \leftarrow \mathcal{G}\left(1^{\kappa}\right)}\left[\mathcal{D}_{2}\left(c k^{\prime}, c, \boldsymbol{y}^{\prime}\right)=1 \mid \begin{array}{r}
(c k, s k) \leftarrow \operatorname{KeyGen}(g k, \boldsymbol{n}, \boldsymbol{K}, \boldsymbol{S}) ; \\
\left(c k^{\prime}, \tau^{\prime}\right) \leftarrow \operatorname{OblKeyGen}\left(g k, \boldsymbol{n}, \boldsymbol{K}, \boldsymbol{S}^{\prime}, c k\right) ; \\
c \leftarrow \mathcal{D}_{1}\left(c k^{\prime}\right) ; \boldsymbol{y}^{\prime} \leftarrow \operatorname{Extract}\left(\tau^{\prime}, c\right), \text { where } s k=(\tau, r)
\end{array}\right]- \\
& \operatorname{Pr}_{g k \leftarrow \mathcal{G}\left(1^{\kappa}\right)}\left[\mathcal{D}_{2}\left(c k, c, y_{S^{\prime}}\right)=1 \mid \begin{array}{r}
(c k, s k) \leftarrow \operatorname{KeyGen}(g k, \boldsymbol{n}, \boldsymbol{K}, \boldsymbol{S}) ; \\
c \leftarrow \mathcal{D}_{1}(c k) ; \boldsymbol{y} \leftarrow \operatorname{Extract}(\tau, c), \text { where } s k=(\tau, r)
\end{array}\right] \mid \leq \operatorname{negl}(\kappa)
\end{aligned}
$$

Next, we show that an SSB commitment scheme with oblivious trapdoor generation is also no-signaling. We leave as an open problem to prove or disprove the opposite implication.

${ }^{13}$ We assume that membership in $C$ is efficiently decidable 
Theorem 2. Let CS = (Setup, KeyGen, OblKeyGen, Com, Extract) be an SSB commitment scheme with oblivious trapdoor generation and h-strong ISH. Then, CS is also h-strong no-signaling.

Proof. Fix any $S^{\prime} \subseteq S \subseteq[n]$ with $|S| \leq K$, and let $\mathcal{D}=\left(\mathcal{D}_{1}, \mathcal{D}_{2}\right)$ be a distinguisher against no signaling extraction for these values. We show by a sequence of games that its success probability is negligible.

$\operatorname{Game}_{0}^{\mathcal{D}}\left(1^{\kappa}\right)$ : In this game, we execute $(c k, s k) \leftarrow \operatorname{KeyGen}(g k, n, K, S)$. We then get $c \leftarrow$ $\mathcal{D}_{1}\left(c k, h_{n s}(\mathrm{sk})\right)$, change it to $\perp$ if $c \notin C$, and compute $y \leftarrow \operatorname{Extract}(\tau, c)$ for $s k=(\tau, r)$. The output is $\mathcal{D}_{2}\left(c k, c, y_{s^{\prime}}\right)$.

$\operatorname{Game}_{1}^{\mathcal{D}}\left(1^{\kappa}\right)$ : In this game, we execute $(c k, s k) \leftarrow \operatorname{KeyGen}(g k, n, K, S)$ and $\left(c k_{\mathrm{obl}}, \tau_{\mathrm{obl}}\right) \leftarrow$ OblKeyGen $\left(g k, \boldsymbol{n}, \boldsymbol{K}, \boldsymbol{S}^{\prime}, c k\right)$. We then compute $h\left(s k_{\mathrm{obl}}\right)$ corresponding to $c k_{\mathrm{obl}}$ and get $c \leftarrow \mathcal{D}_{1}\left(c k_{\mathrm{obl}}, h\left(s k_{\mathrm{obl}}\right)\right)$, change it to $\perp$ if $c \notin C$, and compute $\boldsymbol{y}^{\prime} \leftarrow \operatorname{Extract}\left(\tau_{\mathrm{obl}}, c\right)$. The output is $\mathcal{D}_{2}\left(c k_{\mathrm{obl}}, c, y^{\prime}\right)$.

$\operatorname{Game}_{2}^{\mathcal{D}}\left(1^{\kappa}\right)$ : In this game, we execute $(c k, s k) \leftarrow \operatorname{KeyGen}\left(g k, n, K, S^{\prime}\right)$ and $\left(c k_{\mathrm{obl}}, \tau_{\mathrm{obl}}\right) \leftarrow$ OblKeyGen $\left(g k, n, K, S^{\prime}, c k\right)$. We then compute $h\left(s k_{\text {obl }}\right)$ corresponding to $c k_{\text {obl }}$ and get $c \leftarrow \mathcal{D}_{1}\left(c k_{\mathrm{obl}}, h\left(s k_{\mathrm{obl}}\right)\right)$, change it to $\perp$ if $c \notin C$, and compute $y^{\prime} \leftarrow \operatorname{Extract}\left(\tau_{\mathrm{obl}}, c\right)$. The output is $\mathcal{D}_{2}\left(c k_{\mathrm{obl}}, c, \boldsymbol{y}^{\prime}\right)$.

$\operatorname{Game}_{3}^{\mathcal{D}}\left(1^{\kappa}\right)$ : In this game, we execute $\left(c k^{\prime}, s k^{\prime}\right) \leftarrow \operatorname{KeyGen}\left(g k, n, K, S^{\prime}\right)$. We then get $c \leftarrow \mathcal{D}_{1}\left(c k^{\prime}, h_{n s}\left(s k^{\prime}\right)\right)$, change it to $\perp$ if $c \notin C$, and compute $y \leftarrow \operatorname{Extract}\left(\tau^{\prime}, c\right)$ for $s k=(\tau, r)$. The output is $\mathcal{D}_{2}\left(c k, c, y^{\prime}\right)$.

Now we show the output of games $i$ and $i+1$ is indistinguishable for $i=0$ to 2 .

- Cases $i=0, i=2$. For $i=0$, the two games are distributed identically to the two cases of the oblivious trapdoor generation definition for $S^{\prime} \subseteq S$. Thus, the outputs of the games are statistically close. For $i=2$, the same argument holds for $S=S^{\prime}$. Note that in both cases, the oblivious trapdoor generation distinguisher is unbounded so it can compute $s k_{\mathrm{obl}}$.

- Case $i=1$. The difference in the two games is how we sample the $(c k, s k)$ pair, either programmed to extract $S$ or $S^{\prime}$. By the $h$-index set hiding property the outputs of the two games are computationally indistinguishable.

Finally, noting that $\mathrm{Game}_{0}^{\mathcal{D}}$, $\mathrm{Game}_{3}^{\mathcal{D}}$ correspond to the two cases of no signaling extraction, the result follows.

\subsection{Algebraic SSB Commitments.}

In this section, we define algebraic SSB commitments following the definition of algebraic commitment schemes of [RS20] and extend them to what we call split algebraic SSB commitments.

Informally, an algebraic SSB commitment scheme is a commitment scheme where the commitment key is a matrix [G] of group elements such that (1) committing to a vector $x$ is done by multiplying on the left with $[G]$, that is $[c]=[G] x$ and (2) the trapdoor is a matrix of field elements $\mathbf{T}$ and local extraction is done by multiplying the commitment on the left with $\mathbf{T}^{\top}$, that is $\left[x_{S}\right]=\mathbf{T}^{\top}[c]$. We also allow the commitment key to output some public auxiliary information which is not used in committing nor extraction. 
Definition 5. An SSB commitment scheme CS $=$ (KeyGen, Com, Extract) is algebraic if, given $g k \leftarrow \mathcal{G}\left(1^{\kappa}\right)$, KeyGen $(g k, n, \boldsymbol{K}, S)$ outputs $c k=[\mathbf{G}] \in \mathbb{G}^{\bar{K} \times n}$ and $s k=\left(\mathbf{T} \in \mathbb{Z}_{p}^{\bar{K} \times|S|}, \mathbf{G}\right)$ where $\bar{K} \geq K, \operatorname{Com}([\mathrm{G}], x)=[\mathrm{G}] x$ and $\mathbf{T}^{\top} \mathbf{G}=\Sigma_{S} \mathbf{P}_{S}$.

We also define a subtype of algebraic commitments which are specific to asymmetric groups, where the commitment key is "split" between the two groups.

Definition 6. An SSB commitment scheme CS = (KeyGen, Com, Extract) is split algebraic if KeyGen $(g k, n, \boldsymbol{K}, \mathbf{S})$ outputs $c k=\left([\mathbf{G}]_{1} \in \mathbb{G}_{1}^{\bar{K} \times n},[\mathbf{H}]_{2} \in \mathbb{G}_{2}^{\bar{K} \times n}\right)$ and $s k=\left(\mathbf{T} \in \mathbb{Z}_{p}^{\overline{\mathbf{K}} \times|S|},(\mathbf{G}, \mathbf{H})\right)$, for $\bar{K} \geq \boldsymbol{K}, \operatorname{Com}\left([\mathrm{G}]_{1},[\mathbf{H}]_{2}, \boldsymbol{x}\right)=\left([\mathrm{G}]_{1} x,[\mathbf{H}]_{2} \boldsymbol{x}\right)$ and $\mathbf{T}^{\top} \mathbf{G}+\mathbf{T}^{\top} \mathbf{H}=\boldsymbol{\Sigma}_{S} \mathbf{P}_{S}$.

All SSB commitment schemes in this work are algebraic or split-algebraic. Note that all (split-)SSB commitments only differ on the key generation algorithm. For that reason we sometimes refer to a commitment key distribution as the commitment scheme itself.

In the case of non-split algebraic SSB commitments, we can $\mathbb{G}$-extract by computing

$$
\mathbf{T}^{\top}[c]=\mathbf{T}^{\top}[\mathrm{G} x]=\left[\boldsymbol{\Sigma}_{S} \mathbf{P}_{S} x\right]=\left[x_{S}\right],
$$

while in the case of split-algebraic commitments, we can only $\mathbb{G}_{T}$ extract. That is, we can compute values $\left[\boldsymbol{u}_{S}\right]_{1},\left[\boldsymbol{v}_{S}\right]_{2}$ such that $e\left(\left[\boldsymbol{u}_{S}\right]_{1},[1]_{2}\right)+e\left([1]_{1},\left[\boldsymbol{v}_{S}\right]_{2}\right)=\left[\boldsymbol{x}_{S}\right]_{T}$. Indeed, if $[\boldsymbol{c}]_{1}=[\mathbf{G}]_{1} \boldsymbol{x}$ and $[\boldsymbol{d}]_{2}=[\mathbf{H}]_{2} \boldsymbol{x}$ then we can compute $\left[\boldsymbol{u}_{S}\right]_{1}=\mathbf{T}[\boldsymbol{c}]_{1}$ and $\left[\boldsymbol{v}_{S}\right]_{2}=\mathbf{T}[\boldsymbol{d}]_{2}$ and it holds that

$$
u_{S}+v_{S}=\mathbf{T}^{\top} c+\mathbf{T}^{\top} d=\mathbf{T}^{\top} \mathbf{G} x+\mathbf{T}^{\top} \mathbf{H} x=\left(\mathbf{T}^{\top} \mathbf{G}+\mathbf{T}^{\top} \mathbf{H}\right) \boldsymbol{x}=\boldsymbol{\Sigma}_{S} \mathbf{P}_{S} \boldsymbol{x}=x_{S} .
$$

Note that by definition, if the commitment key generation does not fail, the commitments are perfectly binding/extractable at $S$. This will be the case for commitment schemes with perfect completeness. We will utilize this fact in our constructions to simplify some of the arguments.

\subsection{Somewhere Statistically Binding Commitments with Oblivious Trapdoor Gen- eration}

We present in Fig. 2 a simple construction of an SSB with Oblivious Key Generation from plain SSB commitments with locality parameter 1 . The setup algorithm instantiates $K$ different commitment keys and, given a set $S$, each of the first $|S|$ commitment keys is extractable in a different position $s \in S$. The last $K-|S|$ are binding for the empty set. To commit to a value $x$, one gives $K$ commitments to this value with each of the commitment keys. To verify an opening, one verifies each individual opening and that all the openings are the same.

Note that the ordering of the elements in $S$ is arbitrary and, in some sense, there's no unique key generation algorithm for a set $S$. Indeed, it is only necessary that the commitment key contains $K$ commitment keys for locality 1 such that $c k_{i_{1}}, \ldots, c k_{i_{|S|}}$ are binding at $s_{1}, \ldots, s_{|S|}$ respectively. Note that there are $\left(\begin{array}{c}K \\ |S|\end{array}\right)$ different choices of $i_{1}, \ldots, i_{n}$. For this reason, if the input of the oblivious generator is just $S^{\prime}$, it is impossible to know which commitment keys are the ones corresponding to $S^{\prime}$. To alleviate this, the oblivious key generator receives as advice the indices where $S^{\prime}$ "appears" in $S$ that is, $i_{1}, \ldots, i_{\left|S^{\prime}\right|}$ such that $s_{i_{1}}=s_{1}^{\prime}$.

In this case we need to change a little the proof that oblivious trapdoor generation implies no-signaling. We add a game $\operatorname{Game}_{1 / 2}^{\mathcal{D}}\left(1^{\kappa}\right)$, between games 0 and 1 , which is identical to $\operatorname{Game}_{0}^{\mathcal{D}}\left(1^{\kappa}\right)$ but $\mathcal{E}_{1}$ samples $c k_{i}$ binding at $\left\{s_{i}\right\}$ if $s_{i} \in S^{\prime}$ and at $\emptyset$ if not. By the index-set hiding property of $c k_{1}, \ldots, c k_{K}$ the output of both games is indistinguishable. Game $e_{1}^{\mathcal{D}}\left(1^{\kappa}\right)$ is as before but the oblivious key generator receives also the advise. The rest of the proof is exactly as before 


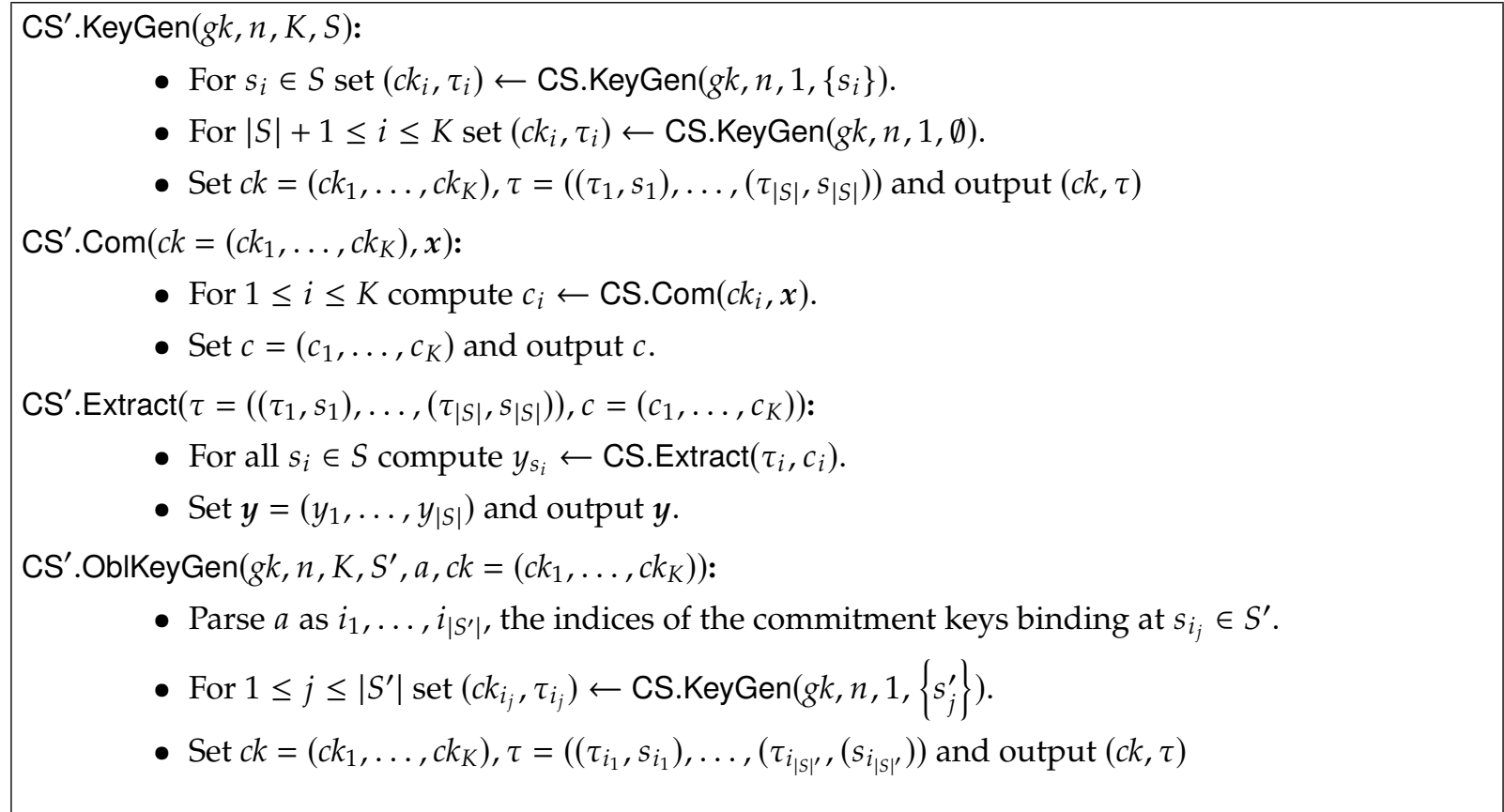

Figure 2: Oblivious SSB commitment scheme from K SSB commitments with locality parameter 1.

Theorem 3. Let CS be an SSB commitment with locality parameter $K=1$. Then construction $\mathrm{CS}^{\prime}$ of Fig. 2 is an SSB commitment with Oblivious Trapdoor Generation.

Proof. First, we show that CS' is an SSB commitment. For index-hiding we can use a standard hybrid argument to show that the concatenation of $K$ commitment keys are indeed indistinguishable. Somewhere Statistical Binding and G-extractability of $\mathrm{CS}^{\prime}$ follow from the respective properties of CS. Indeed, for the former, note that each individual commitment is statistically binding in one coordinate, and for a commitment-opening to verify, all commitments are checked w.r.t. to the same opening; thus, effectively the commitment is statistically binding in the set S. For the latter, we use the same argument and the fact that the extractor of CS can $G$-extract each value independently.

For oblivious trapdoor generation, note that the crs output by OblKeyGen follow exactly the same distribution as the one output by KeyGen as well as a valid trapdoor for $S^{\prime}$.

Next, we present a more efficient SSB commitment scheme with oblivious trapdoor generation. The scheme is parameterized by a group description $g k$, the message space is $\mathbb{Z}_{p}^{n}$ and we extract $\left[x_{S}\right]_{\mu}$. The construction is essentially the one given in [FLPS20], which in turn is a generalization of the so called Multi-Pedersen commitments from [GHR15b], with a minor change in the key generation algorithm. 
$\operatorname{KeyGen}(g k, n, K, S)$ :

- Let $\mathbf{A} \leftarrow \mathcal{D}_{k}, \mathbf{B} \leftarrow \mathbb{Z}_{p}^{K+k \times K-|S|}, \mathbf{W} \leftarrow \mathbb{Z}_{p}^{K-1 \times k+1}$ and define $\mathbf{G}_{0}=\left(\begin{array}{cc}\mathbf{B} & \mathbf{A} \\ & \mathbf{W A}\end{array}\right)$.

- Let $\mathbf{G}_{S} \leftarrow \mathbb{Z}_{p}^{K+k \times|S|}$ and $\Gamma \leftarrow \mathbb{Z}_{p}^{K+k-|S| \times n-|S|}$.

- Let $\mathbf{T}_{S} \in \mathbb{Z}_{p}^{K+k \times|S|}$ s.t. $\mathbf{T}_{S}^{\top} \mathbf{G}_{S}=\mathbf{I}_{|S|}$ and $\mathbf{T}_{S}^{\top} \mathbf{G}_{0}=\mathbf{0}_{|S| \times K+k-|S|}$. Abort if such a matrix does not exist.

- Let $\mathbf{G}=\left(\mathbf{G}_{S} \mid \mathbf{G}_{0} \boldsymbol{\Gamma}\right) \mathbf{P}_{S}$. Output $(c k, s k)=\left([\mathbf{G}]_{\mu},\left(\mathbf{T}_{S}, \mathbf{G}\right)\right)$.

OblKeyGen $\left(g k, n, K, S^{\prime}, c k=[\mathbf{G}]_{\mu}\right): \quad / / S^{\prime} \subseteq S$

- Sample $\mathbf{G}_{1} \leftarrow \mathbb{Z}_{p}^{K+k-\left|S^{\prime}\right| \times\left|S^{\prime}\right|}, \mathbf{G}_{2} \leftarrow \mathbb{Z}_{p}^{\left|S^{\prime}\right| \times\left|S^{\prime}\right|}, \mathbf{R} \leftarrow \mathbb{Z}_{p}^{\left|S^{\prime}\right| \times K+k-\left|S^{\prime}\right|}$.

- Compute a matrix $\mathbf{T} \in \mathbb{Z}_{p}^{\left|S^{\prime}\right| \times\left|S^{\prime}\right|}$ such that $\left(\mathbf{G}_{1}^{\top} \mathbf{R}^{\top}-\mathbf{G}_{2}^{\top}\right) \mathbf{T}=\mathbf{I}_{\left|S^{\prime}\right|}$. Abort if such a matrix does not exist.

- Denote by $\left[\overline{\mathbf{G}}_{\bar{S}^{\prime}}\right]_{\mu}$ the matrix containing the first $K+k-\left|S^{\prime}\right|$ rows of $\left[\mathbf{G}_{\bar{S}^{\prime}}\right]_{\mu}$.

- Output $c k_{\mathrm{ob}}=\left[\mathbf{G}^{*}\right]_{\mu}=\left(\begin{array}{cc}{\left[\mathbf{G}_{1}\right]_{\mu}} & {\left[\overline{\mathbf{G}}_{\bar{S}^{\prime}}\right]_{\mu}} \\ {\left[\mathbf{G}_{2}\right]_{\mu}} & \mathbf{R}\left[\overline{\mathbf{G}}_{\bar{S}^{\prime}}\right]_{\mu}\end{array}\right) \mathbf{P}_{S^{\prime}}$ and $\tau_{\mathrm{ob}}=\mathbf{T}^{*}=\left(\begin{array}{c}\mathbf{R}^{\top} \mathbf{T} \\ -\mathbf{T}\end{array}\right)$

$\operatorname{Com}(c k, x):$ Parse $c k=[\mathrm{G}]_{\mu}$ and output $[c]_{\mu}=[\mathrm{G}]_{\mu} x$.

$\operatorname{Extract}\left(\tau,[c]_{\mu}\right):$ Output $\left[x_{S}\right]_{\mu}=\mathbf{T}_{S}^{\top}[c]_{\mu}$.

Figure 3: SSB commitment scheme with oblivious trapdoor generation parametrized by the matrix distribution $\mathcal{D}_{k}$.

For simplicity, we describe the oblivious key generation algorithm in terms of the permutation $\mathbf{P}_{S}$ while it is not really needed. Indeed, it only needs to randomly sample itself the columns corresponding to $S^{\prime}$ and sample the lower rows as a random combination of upper rows or columns in $\bar{S}^{\prime}$.

In [FLPS20] it is shown that the Index Set Hiding property can be reduced to DDH with a security lost of $2 \log K$ when $\mathbf{G}_{0}$ is uniform using the results of [Vil12]. In our case $\mathbf{G}_{0}$ it is not completely uniform as some part depends on $\mathcal{D}_{k}$. Although it seems still possible to use [Vil12], for simplicity we use a naive hybrid argument at the cost of a less tight reduction. Although the security lost is $2 K$ instead of $2 \log K$, in general $K$ is small (constant in our instantiations) and hence it doesn't make much difference. We give a proof of the following theorem.

Theorem 4. Construction CS of Fig. 3 is an SSB commitment scheme. It is somewhere statistically binding and $\mathbb{G}$-Extractable with probability at least $1-\frac{K}{p}$ and Index Set Hiding with probability at least $1-2 \mathrm{~K} \cdot \operatorname{Adv}_{\mathrm{MDDH}-\mathcal{D}_{k}}(\mathcal{D})$, where $\mathcal{D}$ is a PPT adversary against the $\mathcal{D}_{k}-\mathrm{MDDH}$ assumption.

Proof. We first show that CS.KeyGen aborts only with probability $\frac{K}{p}$. Let $\mathrm{G}_{0}^{\perp}$ be a matrix whose columns are a basis of the kernel of $\mathbf{G}_{0}^{\top}$. Since $\mathbf{G}_{0}$ is uniformly distributed, by the SchwartzZippel lemma, $\mathbf{G}_{0}$ has rank $K+k-|S|$ with probability at least $1-\frac{K+k-|S|}{p}$. Now, consider the matrix $\mathbf{G}_{S}^{\top} \mathbf{G}_{0}^{\perp}$. Again, by the Schwartz-Zippel lemma and the fact that $\mathbf{G}_{S}$ is uniformly distributed, this matrix has rank $|S|$ with probability at least $1-\frac{|S|}{p}$, and thus, it is invertible. Let $\mathbf{T}$ be its inverse. This matrix exists except with probability $\frac{K+k-|S|+|S|}{p}=\frac{K+k}{p}$. Now, set $\mathbf{T}_{S}=\mathbf{G}_{0}^{\perp} \mathbf{T}$. We have that $\mathbf{G}_{S}^{\top} \mathbf{T}_{S}=\mathbf{G}_{S}^{\top} \mathbf{G}_{0}^{\perp} \mathbf{T}^{\prime}=\mathbf{I}_{|S|}$ and $\mathbf{G}_{0}^{\top} \mathbf{T}_{S}=\mathbf{G}_{0}^{\top} \mathbf{G}_{0}^{\perp} \mathbf{T}^{\prime}=\mathbf{0}_{K+k-|S| \times|S|}$, which concludes the proof. 
Index Set Hiding. Consider the following sequence of hybrid games.

- $\mathrm{Game}_{0}^{\mathcal{D}}$ : In this game we sample $(c k, s k) \leftarrow \operatorname{KeyGen}\left(1^{\lambda}, g k, n, K, S_{0}\right)$ and output $\mathcal{D}(c k)$.

- $\mathrm{Game}_{1}^{\mathcal{D}}$ : In this game we sample $(c k, s k) \leftarrow \operatorname{KeyGen}\left(1^{\lambda}, g k, n, K, \emptyset\right)$ and output $\mathcal{D}(c k)$.

- $\mathrm{Game}_{2}^{\mathcal{D}}$ : In this game we sample $(c k, s k) \leftarrow \operatorname{KeyGen}\left(1^{\lambda}, g k, n, K, S_{1}\right)$ and output $\mathcal{D}(c k)$.

Noting that in $\mathrm{Game}_{0}$ and in Game ${ }_{1}$ the difference in the distributions of $c k$ is that in the former $\mathbf{G}_{S_{0}}$ is uniform, while in the later $\mathbf{G}_{S_{0}}=\mathbf{G}_{0} \boldsymbol{\Gamma}_{S_{0}}$, where $\boldsymbol{\Gamma}_{S_{0}} \in \mathbb{Z}_{p}^{K+k-|S| \times\left|S_{0}\right|}$. Using a standard hybrid argument, we can bound the advantage of distinguishing these games by $\left|S_{0}\right| \leq K$ times the advantage of breaking the $\mathbf{G}_{0}-\mathrm{MDDH}$ assumption. It is not hard to see that the $\mathbf{G}_{0}-\mathrm{MDDH}$ can be reduced (without security lost) to the $\mathcal{D}_{k}-\mathrm{MDDH}$ assumption. We conclude that the advantage of distinguishing $\mathrm{Game}_{0}$ and $\mathrm{Game}_{1}$ can be bounded by $K \cdot \mathrm{Adv}_{\mathcal{D}_{k}-\mathrm{MDDH}}$. The same argument applies to $\mathrm{Game}_{1}$ and in Game 2 .

Somewhere Statistically Binding. Finally we show the somewhere statistically binding and extractability property. Let $\mathbf{G}_{S}, \mathbf{G}_{0}, \boldsymbol{\Gamma}$, implicitly defined by $(c k, s k) \leftarrow \mathbf{C S}$. KeyGen $(g k, n, K, S)$. Conditioned on CS.KeyGen not failing, which only happens with probability at most $1-\frac{K}{p}$, the matrix $\mathbf{T}_{S} \in \mathbb{Z}_{p}^{K+k \times|S|}$ satisfies $\mathbf{T}_{S}^{\top} \mathbf{G}=\boldsymbol{\Sigma}_{S} \mathbf{P}_{S}$.

Now let $x, x^{\prime} \in \mathbb{Z}^{n}$. For extractability, note that $\mathrm{T}^{\top} \mathrm{CS} . \operatorname{Com}\left([\mathrm{G}]_{\mu}, x\right)=\mathrm{T}^{\top}[\mathrm{G}]_{\mu} x=\left[\boldsymbol{\Sigma}_{S} \mathbf{P}_{S}\right]_{\mu} x=$ $\left[x_{S}\right]_{\mu}$. Additionally, if CS.com $\left([\mathrm{G}]_{\mu}, x\right)=\mathrm{CS} . \operatorname{com}\left([\mathrm{G}]_{\mu}, x^{\prime}\right)$ and we multiply by $\mathbf{T}^{\top}$ on both sides, we get that $x_{S}=x_{S}^{\prime}$

In the next Theorem we assume $\mathcal{D}_{k}$ outputs full rank matrices with overwhelming probability. Note that this is true for most matrix distributions such as the uniform and the linear family.

Theorem 5. Construction CS of Fig. 3 satisfies Oblivious Trapdoor Generation. Furthermore, for all even unbounded $\mathcal{D}=\left(\mathcal{D}_{1}, \mathcal{D}_{2}\right)$, against oblivious trapdoor generation, $\operatorname{Adv}_{\text {Oblv }}^{\mathrm{CS}}(\mathcal{D}) \leq \frac{K}{p}$.

Proof. Let $K \leq n$ and $S^{\prime} \subseteq S \subseteq[n]$. We first show that the oblivious key follows exactly the same distribution as the original key. Let $c k:=[\mathrm{G}]_{\mu}$ be the output of KeyGen $(g k, n, K, S)$ and $c k_{\mathrm{ob}}=\left[\mathbf{G}^{*}\right]_{\mu}$ be the output of OblKeyGen $\left(g k, n, K, S^{\prime},[\mathbf{G}]\right)$. We can write $c k$ as $\mathbf{G}=$ $\left(\left(\mathbf{G}_{S^{\prime}} \mid \mathbf{G}_{S^{\prime} \mid S}\right) \mathbf{P}_{S^{\prime} \mid S} \mid \mathbf{G}_{0} \mathbf{\Gamma}\right) \mathbf{P}_{S}$.

Let $\overline{\mathbf{G}}_{S^{\prime} \mid S} \in \mathbb{Z}_{p}^{K+k-\left|S^{\prime}\right| \times K-\left|S^{\prime}\right|}, \underline{\mathbf{G}}_{S^{\prime} \mid S} \in \mathbb{Z}_{p}^{\left|S^{\prime}\right| \times K-\left|S^{\prime}\right|}, \overline{\mathbf{G}}_{0} \in \mathbb{Z}_{p}^{K+k-\left|S^{\prime}\right| \times k}, \underline{\mathbf{G}}_{0} \in \mathbb{Z}_{p}^{\left|S^{\prime}\right| \times k}$, such that $\mathbf{G}_{S^{\prime} \mid S}=\left(\begin{array}{l}\overline{\mathbf{G}}_{S^{\prime} \mid S} \\ \underline{\mathbf{G}}_{S^{\prime} \mid S}\end{array}\right), \mathbf{G}_{0}=\left(\begin{array}{l}\overline{\mathbf{G}}_{0} \\ \underline{\mathbf{G}}_{0}\end{array}\right)$. We claim that there exists a matrix $\mathbf{R} \in \mathbb{Z}_{p}^{\left|S^{\prime}\right| \times K+k-\left|S^{\prime}\right|}$, uniformly distributed, such that $\left(\underline{\mathbf{G}}_{S^{\prime} \mid S} \mid \underline{\mathbf{G}}_{0}\right)=\mathbf{R}\left(\overline{\mathbf{G}}_{S^{\prime} \mid S} \mid \overline{\mathbf{G}}_{0}\right)$ as in the output of ObIKeyGen. If this is the case, the distributions of $c k$ output by KeyGen and $c k_{\mathrm{ob}}$ output by OblKeyGen are identical, since we can write

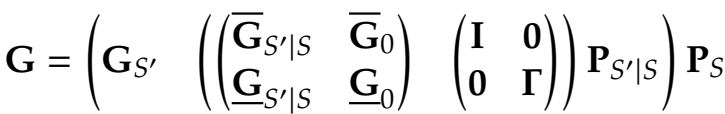

$$
\begin{aligned}
& =\left(\mathbf{G}_{S^{\prime}}\left(\left(\begin{array}{cc}
\overline{\mathbf{G}}_{S^{\prime} \mid S} & \overline{\mathbf{G}}_{0} \\
\mathbf{R}\left(\overline{\mathbf{G}}_{S^{\prime} \mid S}\right. & \left.\overline{\mathbf{G}}_{0}\right)
\end{array}\right)\left(\begin{array}{cc}
\mathbf{I} & \mathbf{0} \\
\mathbf{0} & \boldsymbol{\Gamma}
\end{array}\right)\right) \mathbf{P}_{S^{\prime} \mid S}\right) \mathbf{P}_{S} \\
& =\left(\begin{array}{lc}
\mathbf{G}_{S^{\prime}} & \overline{\mathbf{G}}_{\bar{S}} \\
& \mathbf{R} \overline{\mathbf{G}}_{\bar{S}}
\end{array}\right) \mathbf{P}_{S} .
\end{aligned}
$$


First we show that the matrix $\left(\overline{\mathbf{G}}_{S^{\prime} \mid} \mid \overline{\mathbf{G}}_{0}\right)$ is full rank with overwhelming probability. Indeed, $\overline{\mathbf{G}}_{0}=\left(\frac{\mathbf{A}}{\mathbf{W}_{\mathbf{A}}}\right)$, where $\mathbf{A} \leftarrow \mathcal{D}_{k}, \overline{\mathbf{W}} \leftarrow \mathbb{Z}_{p}^{K-1-\left|S^{\prime}\right| \times k+1}$, and it has rank $k$. By the fact that $\overline{\mathbf{G}}_{S^{\prime} \mid S}$ is uniform, using the Schwartz-Zippel lemma we get that $\left(\overline{\mathbf{G}}_{S^{\prime} \mid S} \mid \overline{\mathbf{G}}_{0}\right)$ has rank $K+k-\left|S^{\prime}\right|$ except with probability $\frac{K-\left|S^{\prime}\right|}{p}$. This means that the matrix is invertible and we can set $\mathbf{R}=$ $\left(\underline{\mathbf{G}}_{S^{\prime} \mid S} \mid \underline{\mathbf{G}}_{0}\right)\left(\overline{\mathbf{G}}_{S^{\prime} \mid S} \mid \overline{\mathbf{G}}_{0}\right)^{-1}$. Furthermore, both $\underline{\mathbf{G}}_{S^{\prime} \mid S}$ and $\underline{\mathbf{G}}_{0}=\underline{\mathbf{W}} \mathbf{A}$ are uniform, the latter since $\underline{\mathbf{W}} \in \mathbb{Z}_{p}^{\left|S^{\prime}\right| \times k+1}$ is uniformly distributed and $\mathbf{A}$ is full rank, and the former by construction.

To conclude the proof, we to show that the trapdoor output by ObIKeyGen $\left(g k, n, K, S^{\prime},[\mathrm{G}]\right)$ is correct w.r.t $c k_{\mathrm{ob}}$, that is $\mathbf{T}^{* \top} \mathbf{G}^{*}=\Sigma_{S^{\prime}}$. By a simple calculation,

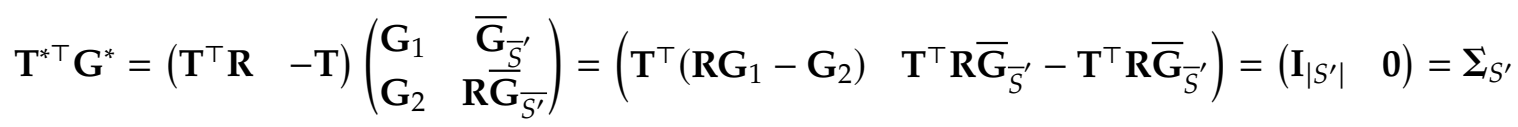

where $\mathbf{T}^{\top}\left(\mathbf{R G}_{1}-\mathbf{G}_{2}\right)=\mathbf{I}_{S^{\prime}}$ by construction.

In the next sections we assume that KeyGen and OblKeyGen do not abort. This is w.l.o.g. since we can always re-sample values when an abort happens. Note that in this case, the keys of both KeyGen and OblKeyGen are "somewhere perfectly binding".

\subsection{Kronecker Product of two SSB commitments}

Let $\mathrm{CS}$ be an algebraic commitment scheme and let $[\mathbf{G}]_{1} \in \mathbb{G}_{1}^{\ell_{1} \times n_{1}}$ and $[\mathbf{H}]_{2} \in \mathbb{G}_{2}^{\ell_{2} \times n_{2}}$ commitment keys. We note there's the following key and input homomorphism

$$
\text { CS.Com }\left([\mathrm{G}]_{1}, x\right) \otimes \mathrm{CS} . \operatorname{Com}\left([\mathbf{H}]_{2}, y\right)=\mathrm{CS} . \operatorname{Com}\left([\mathrm{G} \otimes \mathbf{H}]_{T}, x \otimes y\right),
$$

where $\otimes$ is the Kronecker product and is naturally defined w.r.t. the pairing function when the operands are group elements. To get a structure preserving primitive, so that we can later efficiently show that committed values satisfy some relation, it is better to consider all keys defined over one of the base groups [AFG+16]. However, as noted in [GHR15b], in asymmetric groups it is not clear whether $[\mathbf{G} \otimes \mathbf{H}]_{1}\left(\right.$ or $\left.[\mathbf{G} \otimes \mathbf{H}]_{2}\right)$ defines an SSB commitment. Indeed, if we use the ISH of $\mathrm{CS}_{2}$ to prove that $[\mathbf{G} \otimes \mathbf{H}]_{1}$ is ISH, it turns out that we only know $[\mathbf{H}]_{2}$ in group $\mathbb{G}_{2}$ and hence we can only compute $\mathbf{G} \otimes[\mathbf{H}]_{2}$ which is trivially distinguishable from the original key. To overcome this problem, the authors in [GHR15b] used the split key $\left[\mathbf{Q}_{1}\right]_{1}=[\mathbf{G} \otimes \mathbf{H}+\mathbf{Z}]_{1} \in \mathbb{G}_{1}^{\ell_{1} \ell_{2} \times n_{1} n_{2}},\left[\mathbf{Q}_{2}\right]_{2}=[-\mathbf{Z}] \in \mathbb{G}_{2}^{\ell_{1} \ell_{2} \times n_{1} n_{2}}$, for $\mathbf{Z} \leftarrow \mathbb{Z}_{p}^{\ell_{1} \ell_{2} \times n_{1} n_{2}}$. In this case we can write the homomorphism as follows

$$
\begin{aligned}
& \text { CS.Com }\left([\mathrm{G}]_{1}, x\right) \otimes \text { CS.Com }\left([\mathbf{H}]_{2}, y\right)= \\
& \quad e\left(\operatorname{CS} . \operatorname{Com}\left(\left[\mathbf{Q}_{1}\right]_{1}, x \otimes y\right),[1]_{2}\right)+e\left([1]_{1}, \operatorname{CS} . \operatorname{Com}\left(\left[\mathbf{Q}_{2}\right]_{2}, x \otimes y\right) .\right.
\end{aligned}
$$

If additionally CS is an instance of the scheme defined in figure 3, the following theorem holds.

Theorem 6. For $n_{i} \in \mathbb{N}, K_{i} \leq n_{i}, S_{i} \subseteq\left[n_{i}\right]$ and $\left|S_{i}\right| \leq K_{i}$, let $\mathrm{CS}_{1}$ and $\mathrm{CS}_{2}$ be two instances of the SSB commitment of figure 3 such that $\left(c k_{i}, s k_{i}\right) \leftarrow \mathrm{CS}_{i} . \mathrm{KGen}\left(g k_{i}, m_{i}, K_{i}, S_{i}\right)$ outputs a key over $\mathbb{G}_{i}$, where $i \in\{1,2\}$. Then the commitment scheme kCS, where kCS.KGen $\left(g k,\left(n_{1}, n_{2}\right),\left(K_{1}, K_{2}\right),\left(S_{1}, S_{2}\right)\right)$ is defined as

kCS.KGen $\left(g k, c k_{1}, c k_{2}, s k_{1}, s k_{2}\right): / /\left(c k_{i}, s k_{i}\right) \leftarrow \mathrm{CS}_{1} \cdot \operatorname{KGen}\left(g k, m_{i}, K_{i}, S_{i}\right)$

1. Parse $s k_{1}$ as $\left(\mathbf{G}, \mathbf{T}_{\mathbf{G}}\right)$ and $s k_{2}$ as $\left(\mathbf{H}, \mathbf{T}_{\mathbf{H}}\right)$.

2. Let $\mathbf{Q}_{1}=\mathbf{G} \otimes \mathbf{H}+\mathbf{Z}$ and $\mathbf{Q}_{2}=-\mathbf{Z}$, where $\mathbf{Z} \leftarrow \mathbb{Z}_{p}^{\bar{K}_{1} \bar{K}_{2} \times n_{1} n_{2}}$. 
3. Let $\mathbf{T}_{\mathbf{Q}}=\mathbf{T}_{\mathbf{G}} \otimes \mathbf{T}_{\mathbf{H}}$ and $\mathrm{aux}=\left(c k_{1}, c k_{2}\right)$.

4. output $c k=\left(\left[\mathbf{Q}_{1}\right]_{1},\left[\mathbf{Q}_{2}\right]_{2}\right.$, aux $)$ and $s k=\left(\mathbf{T}_{\mathbf{Q}}, \mathbf{Q}_{1}, \mathbf{Q}_{2}, \mathbf{G}, \mathbf{H}\right)$.

is a split algebraic oblivious SSB commitment scheme.

Proof. Index Set Hiding. Let $S_{1}, S_{1}^{\prime} \subseteq\left[n_{1}\right],\left|S_{1}\right|,\left|S_{1}^{\prime}\right| \leq K_{1}$ and $S_{2}$. The result follows from the indistinguishability of the following distributions (this is essentially part of the proof in [GHR15a, Theorem 6]). For simplicity we write $\mathbf{X} \leftarrow$ CS.KGen, where $\mathbf{X}$ is some part of $(c k, s k)$, meaning that after running KGen we discard everything but $\mathbf{X}$. Recall that aux $=\left([\mathbf{G}]_{1},[\mathbf{H}]_{2}\right)$.

1. aux, $[\mathbf{G} \otimes \mathbf{H}+\mathbf{Z}]_{1},[-\mathbf{Z}]_{2}, \quad \mathbf{G} \leftarrow \mathbf{C S} \cdot \operatorname{Setup}\left(g k, n_{1}, K_{1}, S_{1}\right), \mathbf{H} \leftarrow \operatorname{CS} \cdot \operatorname{Setup}\left(g k, n_{2}, K_{2}, S_{2}\right)$,

2. aux, $[\mathbf{G}]_{1} \otimes \mathbf{H}+[\mathbf{Z}]_{1},[-\mathbf{Z}]_{2}, \mathbf{G} \leftarrow \mathbf{C S} \cdot \operatorname{Setup}\left(g k, n_{1}, K_{1}, S_{1}\right), \mathbf{H} \leftarrow \operatorname{CS} \cdot \operatorname{Setup}\left(g k, n_{2}, K_{2}, S_{2}\right)$,

3. aux, $[\mathbf{G}]_{1} \otimes \mathbf{H}+[\mathbf{Z}]_{1},[-\mathbf{Z}]_{2}, \mathbf{G} \leftarrow \mathbf{C S} \cdot \operatorname{Setup}\left(g k, n_{1}, K_{1}, S_{1}^{\prime}\right), \mathbf{H} \leftarrow \operatorname{CS} \cdot \operatorname{Setup}\left(g k, n_{2}, K_{2}, S_{2}\right)$,

4. aux, $[\mathbf{Z}]_{1}, \mathbf{G} \otimes[\mathbf{H}]_{2}-[\mathbf{Z}]_{2}, \quad \mathbf{G} \leftarrow \mathbf{C S} \cdot \operatorname{Setup}\left(g k, n_{1}, K_{1}, S_{1}^{\prime}\right), \mathbf{H} \leftarrow \operatorname{CS} \cdot \operatorname{Setup}\left(g k, n_{2}, K_{2}, S_{2}\right)$,

5. aux, $[\mathbf{Z}]_{1}, \mathbf{G} \otimes[\mathbf{H}]_{2}-[\mathbf{Z}]_{2}, \quad \mathbf{G} \leftarrow \mathbf{C S} \cdot \operatorname{Setup}\left(g k, n_{1}, K_{1}, S_{1}^{\prime}\right), \mathbf{H} \leftarrow \operatorname{CS} \cdot \operatorname{Setup}\left(g k, n_{2}, K_{2}, S_{2}^{\prime}\right)$,

6. aux, $[\mathbf{G} \otimes \mathbf{H}+\mathbf{Z}]_{1},[-\mathbf{Z}]_{2}, \quad \mathbf{G} \leftarrow \mathbf{C S} \cdot \operatorname{Setup}\left(g k, n_{1}, K_{1}, S_{1}^{\prime}\right), \mathbf{H} \leftarrow \operatorname{CS} \cdot \operatorname{Setup}\left(g k, n_{2}, K_{2}, S_{2}^{\prime}\right)$.

Perfect indistinguishability between distributions 1-2, 3-4 and 5-6 follows from the fact that always both distributions are uniformly distributed conditioned on their sum being equal to $\mathbf{G} \otimes \mathbf{H}$. On the other hand, computational indistinguishability of distributions 2-3 and 4-5 follows from the ISH of $\mathrm{CS}_{1}$ and $\mathrm{CS}_{2}$ respectively.

Somewhere Statistically Binding and G-Extractability. Let $z, z^{\prime} \in \mathbb{Z}_{p}^{n_{1} n_{2}}$ such thatkCS.Com $(c k, z)=$ kCS.Com $\left(c k, z^{\prime}\right)$. Let $\mathbf{T}_{\mathbf{G}}$ and $\mathbf{T}_{\mathbf{H}}$ the trapdoors associated to $[\mathrm{G}]_{1}$ and $[\mathbf{H}]_{2}$, respectively, then

$$
\begin{aligned}
0 & =\left(\mathbf{T}_{\mathbf{G}} \otimes \mathbf{T}_{\mathbf{H}}\right)(\mathbf{G} \otimes \mathbf{H}+\mathbf{Z})\left(z-z^{\prime}\right)-\left(\mathbf{T}_{\mathbf{G}} \otimes \mathbf{T}_{\mathbf{H}}\right) \mathbf{Z}\left(z-z^{\prime}\right) \\
& =\left(\mathbf{T}_{\mathbf{G}} \otimes \mathbf{T}_{\mathbf{H}}\right)(\mathbf{G} \otimes \mathbf{H})\left(z-z^{\prime}\right) \\
& =\left(\mathbf{T}_{\mathrm{G}} \mathbf{G}\right) \otimes\left(\mathbf{T}_{\mathbf{H}} \mathbf{H}\right)\left(z-z^{\prime}\right) \\
& =\left(\boldsymbol{\Sigma}_{S_{1}} \mathbf{P}_{S_{1}}\right) \otimes\left(\boldsymbol{\Sigma}_{S_{2}} \mathbf{P}_{S_{2}}\right)\left(z-z^{\prime}\right) \\
& =\left(\boldsymbol{\Sigma}_{S_{1}} \otimes \mathbf{\Sigma}_{S_{2}}\right)\left(\mathbf{P}_{S_{1}} \otimes \mathbf{P}_{S_{2}}\right)\left(z-z^{\prime}\right) \\
& =z_{S_{1}, S_{2}}-z_{S_{1}, S_{2}}^{\prime}
\end{aligned}
$$

Note that this also shows that the trapdoors correctly extracts $\left[z_{S_{1}, S_{2}}\right]_{T}$ from kCS.Com $(c k, z)$.

Oblivious Trapdoor Generation. We first recall the following commutative property of kronecker products.

Fact 3. For every $m_{1}, m_{2}, n_{1}, n_{2} \in \mathbb{N}$ there exists permutation matrices $\Pi_{1} \in\{0,1\}^{m_{1} n_{1} \times m_{1} n_{1}}, \boldsymbol{\Pi}_{2} \in$ $\{0,1\}^{m_{2} n_{2} \times m_{2} n_{2}}$ such that for any pair of matrices $\mathbf{M} \in \mathbb{Z}_{p}^{m_{1} \times m_{2}}, \mathbf{N} \in \mathbb{Z}_{p}^{n_{1} \times n_{2}}$ it holds that $\mathbf{M} \otimes \mathbf{N}=$ $\Pi_{1}(\mathbf{N} \otimes \mathbf{M}) \Pi_{2}$. Note that $\Pi_{1}$ and $\Pi_{2}$ depend only on the size of $\mathbf{M}$ and $\mathbf{N}$ but not the values of their entries.

We construct an oblivious key generation algorithm as follows.

kCS.OblKeyGen $\left(g k,\left(n_{1}, n_{2}\right),\left(K_{1}, K_{2}\right),\left(S_{1}, S_{2}\right), c k\right)$ :

1. Parse $c k$ as $\left[\mathbf{Q}_{1}\right]_{1},\left[\mathbf{Q}_{2}\right]_{2}$ and aux $=\left([\mathbf{G}]_{1},[\mathbf{H}]_{2}\right)$. 
2. Run

$$
\begin{aligned}
\left(\left[\mathbf{G}^{*}\right], \mathbf{T}_{1}\right) & \leftarrow \mathrm{CS}_{1} \text {.OblKeyGen }\left(g k, n_{1}, K_{1}, S_{1},[\mathbf{G}]_{1}\right) \\
\left(\left[\mathbf{H}^{*}\right]_{2}, \mathbf{T}_{2}\right) & \leftarrow \mathrm{CS}_{2} . \text { ObIKeyGen }\left(g k, m_{2}, K_{2}, S_{2},[\mathbf{H}]_{2}\right)
\end{aligned}
$$

and use the random coins of OblKeyGen to retrieve $\mathbf{G}_{S_{1}}^{*}, \mathbf{R}_{1}$ and $\mathbf{H}_{S_{1}}^{*}, \mathbf{R}_{2}$ such that

$$
\left[\mathbf{G}^{*}\right]_{1}=\left(\begin{array}{cc}
{\left[\mathbf{G}_{S_{1}}^{*}\right]_{1}} & {\left[\overline{\mathbf{G}}_{\bar{S}_{1}}\right]_{1}} \\
& \mathbf{R}_{1}\left[\overline{\mathbf{G}}_{\bar{S}_{1}}\right]_{1}
\end{array}\right) \mathbf{P}_{S_{1}} \text { and } \mathbf{H}^{*}=\left(\begin{array}{cc}
{\left[\mathbf{H}_{S_{2}}^{*}\right]_{2}} & {\left[\overline{\mathbf{H}}_{\bar{S}_{2}}\right]_{2}} \\
& \mathbf{R}_{2}\left[\overline{\mathbf{H}}_{\bar{S}_{2}}\right]_{2}
\end{array}\right) \mathbf{P}_{S_{2}},
$$

as defined in Fig. 3.

3. Let $\left[\mathbf{A}_{1}\right]_{1},\left[\mathbf{A}_{2}\right]_{2}$ be the matrices containing the first $\left(K_{1}+k-\left|S_{1}\right|\right)\left(K_{2}+k\right)$ rows of $\left[\left(\mathbf{Q}_{1}\right)_{\bar{S}_{1}, \bar{S}_{2}}\right]_{1}$ and $\left[\left(\mathbf{Q}_{2}\right)_{\bar{S}_{1}, \bar{S}_{2}}\right]_{2}$, respectively.

4. Let $\Pi_{1}$ and $\Pi_{2}$ the permutation matrices of Fact 3 for matrices with $\left(K_{1}+k-|S|_{1}\right)$ and $\left(K_{2}+k\right)$ rows, and $n_{1}-\left|S_{1}\right|$ and $n_{2}-\left|S_{2}\right|$ columns.

5. Define $\left[\mathbf{B}_{1}\right]_{1}$ and $\left[\mathbf{B}_{2}\right]_{2}$ be the matrices of the first $\left(K_{1}+k-\left|S_{1}\right|\right)\left(K_{2}+k-\left|S_{2}\right|\right)$ columns of $\boldsymbol{\Pi}_{1}^{\top}\left[\mathbf{A}_{1}\right]_{1} \boldsymbol{\Pi}_{2}^{\top}$ and $\boldsymbol{\Pi}_{1}^{\top}\left[\mathbf{A}_{2}\right]_{2} \boldsymbol{\Pi}_{2}^{\top}$, respectively.

6. Let $\left[\mathbf{A}_{1}^{*}\right]_{1}=\boldsymbol{\Pi}_{1}\left(\begin{array}{c}{\left[\mathbf{B}_{1}\right]_{1}} \\ \left(\mathbf{R}_{2} \otimes \mathbf{I}_{K_{1}+k-\left|S_{1}\right|}\right)\left[\mathbf{B}_{1}\right]_{1}\end{array}\right) \boldsymbol{\Pi}_{2}$ and $\left[\mathbf{A}_{2}^{*}\right]_{2}=\boldsymbol{\Pi}_{1}\left(\begin{array}{c}{\left[\mathbf{B}_{2}\right]_{2}} \\ \left(\mathbf{R}_{2} \otimes \mathbf{I}_{K_{1}+k-\left|S_{1}\right|}\right)\left[\mathbf{B}_{2}\right]_{2}\end{array}\right) \boldsymbol{\Pi}_{2}$.

7. Pick $\mathbf{Z} \leftarrow \mathbb{Z}_{p}^{\left(K_{1}+k\right)\left(K_{2}+k\right) \times n_{1} n_{2}}$ and let

$$
\begin{aligned}
& {\left[\mathbf{Q}_{1}^{*}\right]_{1}=\left(\left[\mathbf{Z}_{S_{1},\left[n_{2}\right]}\right]_{1}\left|\left[\mathbf{G}_{\bar{S}_{1}}^{*}\right]_{1} \otimes \mathbf{H}_{S_{2}}^{*}+\left[\mathbf{Z}_{\bar{S}_{1}, S_{2}}\right]_{1}\right|\left(\begin{array}{c}
{\left[\mathbf{A}_{1}^{*}\right]_{1}} \\
\left(\mathbf{R}_{1} \otimes \mathbf{I}_{K_{2}+k}\right)\left[\mathbf{A}_{1}^{*}\right]_{1}
\end{array}\right)+\left[\mathbf{Z}_{\bar{S}_{1}, \bar{S}_{2}}\right]_{1}\right)\left(\mathbf{P}_{S_{1}} \otimes \mathbf{P}_{S_{2}}\right)} \\
& {\left[\mathbf{Q}_{2}^{*}\right]_{2}=\left(\mathbf{G}_{S_{1}}^{*} \otimes\left[\mathbf{H}^{*}\right]_{2}-\left[\mathbf{Z}_{S_{1},\left[n_{2}\right]}\right]_{1}\left|-\left[\mathbf{Z}_{\bar{S}_{1}, S_{2}}\right]_{2}\right|\left(\begin{array}{c}
{\left[\mathbf{A}_{2}^{*}\right]_{2}} \\
\left(\mathbf{R}_{1} \otimes \mathbf{I}_{K_{2}+k}\right)\left[\mathbf{A}_{2}^{*}\right]_{2}
\end{array}\right)-\left[\mathbf{Z}_{\bar{S}_{1}, \bar{S}_{2}}\right]_{2}\right)\left(\mathbf{P}_{S_{1}} \otimes \mathbf{P}_{S_{2}}\right)}
\end{aligned}
$$

8. Let aux $=\left(\left[\mathbf{G}^{*}\right]_{1},\left[\mathbf{H}^{*}\right]_{2}\right)$ and $\mathbf{T}=\mathbf{T}_{1} \otimes \mathbf{T}_{2}$.

9. Return $\left(c k=\left(\left[\mathbf{Q}_{1}^{*}\right]_{1},\left[\mathbf{Q}_{2}^{*}\right]_{2}\right.\right.$, aux $\left.), \tau=\mathbf{T}\right)$.

Now we show that $c k$ is correctly distributed. Since $\mathrm{CS}_{1}$ and $\mathrm{CS}_{2}$ are both oblivious SSB commitments, it holds that aux $=\left[\mathbf{G}^{*}\right]_{1},\left[\mathbf{H}^{*}\right]$ follows the same distribution as the honest aux. It is enough to show that $\mathbf{Q}^{*}=\mathbf{Q}_{1}^{*}+\mathbf{Q}_{2}^{*}=\mathbf{G}^{*} \otimes \mathbf{H}^{*}$. This is the case since if this holds, the commitment key $\left[\mathbf{Q}_{1}^{*}\right]_{1},\left[\mathbf{Q}_{2}^{*}\right]_{2}$ consists of two uniform matrices, conditioned on their sum equaling $\mathbf{G}^{*} \otimes \mathbf{H}^{*}$, and this is the distribution of the honest key as well.

It is clear that this is the case for $\mathbf{Q}_{S_{1},\left[n_{2}\right]}^{*}$ and $\mathbf{Q}_{\bar{S}_{1}, S_{2}}^{*}$, so we show it is also the case for $\mathbf{Q}_{\bar{S}_{1}, \bar{S}_{2}}$. First, note that $\mathbf{Q}_{\bar{S}_{1}, \bar{S}_{2}}=\left(\mathbf{Q}_{1}+\mathbf{Q}_{2}\right)_{\bar{S}_{1}, \bar{S}_{2}}=\left(\begin{array}{l}\overline{\mathbf{G}}_{\bar{S}_{1}} \otimes \mathbf{H}_{\bar{S}_{2}} \\ \underline{\mathbf{G}}_{\bar{S}_{1}} \otimes \mathbf{H}_{\bar{S}_{2}}\end{array}\right)$ and then $\mathbf{A}=\mathbf{A}_{1}+\mathbf{A}_{2}=\overline{\mathbf{G}}_{\bar{S}_{1}} \otimes \mathbf{H}_{\bar{S}_{2}}$. It follows that $\boldsymbol{\Pi}_{1}^{\top} \mathbf{A} \Pi_{2}^{\top}=\boldsymbol{\Pi}_{1}^{\top} \boldsymbol{\Pi}_{1} \mathbf{H}_{\bar{S}_{2}} \otimes \overline{\mathbf{G}}_{\bar{S}_{1}} \boldsymbol{\Pi}_{2} \boldsymbol{\Pi}_{2}^{\top}=\left(\begin{array}{c}\overline{\mathbf{H}}_{\bar{S}_{2}} \otimes \overline{\mathbf{G}}_{\bar{S}_{1}} \\ \underline{\mathbf{H}}_{\bar{S}_{2}} \otimes \overline{\mathbf{G}}_{\bar{S}_{1}}\end{array}\right)$ and hence $\mathbf{B}=\mathbf{B}_{1}+\mathbf{B}_{2}=\overline{\mathbf{H}}_{\bar{S}_{2}} \otimes \overline{\mathbf{G}}_{\bar{S}_{1}}$. 
Finally we have that

$$
\begin{aligned}
& \mathbf{Q}_{\bar{S}_{1}, \bar{S}_{2}}^{*}=\left(\begin{array}{c}
\mathbf{A}_{1}^{*}+\mathbf{A}_{2}^{*} \\
\left(\mathbf{R}_{1} \otimes \mathbf{I}_{K_{2}+k}\right)\left(\mathbf{A}_{1}^{*}+\mathbf{A}_{2}^{*}\right)
\end{array}\right)=\left(\begin{array}{c}
\boldsymbol{\Pi}_{1}\left(\begin{array}{c}
\mathbf{B}_{1}+\mathbf{B}_{2} \\
\left(\mathbf{R}_{2} \otimes \mathbf{I}_{K_{1}+k-\left|S_{1}\right|}\right)\left(\mathbf{B}_{1}+\mathbf{B}_{2}\right)
\end{array}\right) \boldsymbol{\Pi}_{2} \\
\left(\mathbf{R}_{1} \otimes \mathbf{I}_{K_{2}+k}\right)\left(\mathbf{A}_{1}^{*}+\mathbf{A}_{2}^{*}\right)
\end{array}\right) \\
& =\left(\begin{array}{c}
\left.\boldsymbol{\Pi}_{1}\left(\begin{array}{c}
\overline{\mathbf{H}}_{\bar{S}_{2}} \otimes \overline{\mathbf{G}}_{\bar{S}_{1}} \\
\left(\mathbf{R}_{2} \otimes \mathbf{I}_{K_{1}+k-\left|S_{1}\right|}\right)\left(\overline{\mathbf{H}}_{\bar{S}_{2}} \otimes \overline{\mathbf{G}}_{\bar{S}_{1}}\right)
\end{array}\right) \boldsymbol{\Pi}_{2}\right)=\left(\begin{array}{c}
\boldsymbol{\Pi}_{1}\left(\begin{array}{c}
\overline{\mathbf{H}}_{\bar{S}_{2}} \\
\mathbf{R}_{2} \overline{\mathbf{H}}_{\bar{S}_{2}}
\end{array}\right) \otimes \overline{\mathbf{G}}_{\bar{S}_{1}} \boldsymbol{\Pi}_{2} \\
\left(\mathbf{R}_{1} \otimes \mathbf{I}_{K_{2}+k}\right)\left(\mathbf{A}_{1}^{*}+\mathbf{A}_{2}^{*}\right)
\end{array}\right)\left(\mathbf{I}_{K_{2}+k}\right)\left(\mathbf{A}_{1}^{*}+\mathbf{A}_{2}^{*}\right)
\end{array}\right) \\
& =\left(\begin{array}{c}
\boldsymbol{\Pi}_{1} \mathbf{H}_{\bar{S}_{2}}^{*} \otimes \overline{\mathbf{G}}_{\bar{S}_{1}} \boldsymbol{\Pi}_{2} \\
\left(\mathbf{R}_{1} \otimes \mathbf{I}_{K_{2}+k}\right)\left(\mathbf{A}_{1}^{*}+\mathbf{A}_{2}^{*}\right)
\end{array}\right)=\left(\begin{array}{c}
\overline{\mathbf{G}}_{\bar{S}_{1}} \otimes \mathbf{H}_{\bar{S}_{2}}^{*} \\
\left(\mathbf{R}_{1} \otimes \mathbf{I}_{K_{2}+k}\right)\left(\overline{\mathbf{G}}_{\bar{S}_{1}} \otimes \mathbf{H}_{\bar{S}_{2}}^{*}\right)
\end{array}\right) \\
& =\mathbf{G}_{\bar{S}_{1}}^{*} \otimes \mathbf{H}_{\bar{S}_{2}}^{*} \text {. }
\end{aligned}
$$

For finishing the proof it suffices to show that the rest of the input given to the distinguisher is correctly distributed. Note that, following definition 4 and fact 2, $\left[\boldsymbol{y}_{S_{1}^{\prime}, S_{2}^{\prime}}\right]_{T}=$ $\left(\operatorname{Extract}\left(\mathbf{T}_{S_{1}, S_{2}},[\boldsymbol{c}]_{1},[\boldsymbol{d}]_{2}\right)\right)_{S_{1}^{\prime}, S_{2}^{\prime}}=\left[z_{S_{1}^{\prime}, S_{2}^{\prime} \mid S_{1}^{\prime}, S_{2}}\right]_{T}=\left[z_{S_{1}^{\prime}, S_{2}^{\prime}}\right]_{T}=\operatorname{Extract}\left(\mathbf{T}_{S_{1}^{\prime}, S_{2}^{\prime}},[\boldsymbol{c}]_{1},[\boldsymbol{d}]_{2}\right)$.

Corollary 1. Construction from fig. 3 instantiated in $\mathbb{G}_{1}$ is ISH even when the adversary is given $h(s k)=\left([\mathbf{H}]_{2},[\mathbf{G} \otimes \mathbf{H}+\mathbf{Z}]_{1},[-\mathbf{Z}]_{2}\right)$. Similarly, it is also ISH when instantiated in $\mathbb{G}_{2}$ when the adversary is given $h(s k)=\left([\mathbf{G}]_{1},[\mathbf{G} \otimes \mathbf{H}+\mathbf{Z}]_{1},[-\mathbf{Z}]_{2}\right)$.

Proof. Follows directly from the ISH of the kronecker SSB commitment of Theorem 6. Specifically, ISH for $\mathbb{G}_{1}$ follows from the indistinguishability of distributions 1 to 3 from Theorem 6, and ISH for $\mathbb{G}_{2}$ follows from the indistinguishability of distributions 3 to 6 .

\section{Quasi-Arguments with Preprocessing}

In this section we introduce an extension of Quasi Arguments as defined in [KPY19] which adds support for language dependent crs or preprocessing such as the so called QA-NIZK arguments [JR13]. Additionally we use different languages for completeness and local soundness, i.e. promise problems, to incorporate the "knowledge transfer" soundness of [GR19].

Following [JR13], languages are parametrized by $\rho \in \mathcal{L}_{\text {par }}$ and $\rho$ sampled from some distribution $\mathcal{D}_{\text {par }}$. We say tat $\mathcal{D}_{\text {par }}$ is witness samplable if $\rho$ can be efficiently sampled together with a witness $\theta$ for $\rho \in \mathcal{L}_{\text {par }}$. We simply write $(\theta, \rho) \leftarrow \mathcal{D}_{\text {par. }}$. Each $\rho \in \mathcal{L}_{\text {par }}$ defines a language $\mathcal{L}_{\rho}$ with the corresponding relations $\mathcal{R}_{\rho}^{\text {yes }}$, that is $\mathcal{L}_{\rho}=\left\{x \mid \exists w\right.$ s.t. $\left.(x, w) \in \mathcal{R}_{\rho}^{\text {yes }}\right\}$. After the language is fixed there is a (language dependent) prepossessing stage where a common reference string is generated. Going a step forward, we would like our statements to be commitments and that $\mathcal{R}_{\rho}^{\text {yes }}$ puts some restriction on the commitment opening. Since we will be using SSB commitments, the language parameter must contain the SSB commitment key. Therefore, we assume distribution $\mathcal{D}_{\text {par }}$ receives as input $d \in \mathbb{N}$ (the size of the opening), a locality parameter $K \leq d$ and a set $S \subseteq[d]$. It will be useful to define $\mathcal{L}_{\rho}^{\text {yes }}=\mathcal{L}_{\rho}$ and $\mathcal{L}_{\rho}^{\text {no }}$ the complement of $\mathcal{L}_{\rho}^{\text {yes }}$, and similarly define $\mathcal{R}_{\rho}^{\text {yes }}$ and $\mathcal{R}_{\rho}^{\text {no }}$. Traditional arguments of knowledge require that from any accepting statement and proof pair one can extract a witness $w$ such that $(x, w) \in \mathcal{R}_{\rho}^{\text {no }}$ only with negligible probability.In a quasi-argument of knowledge only a small part of the witness $w_{S}$ is extracted and $\left(x, w_{S}\right) \in \mathcal{R}_{\rho, S}^{\text {yes }}$ with overwhelming probability, where $\mathcal{R}_{\rho, S}^{\text {yes }}$ is a "local version" of $\mathcal{R}_{\rho}^{\text {yes }} .{ }^{14}$

\footnotetext{
${ }^{14}$ In the case $x$ is a 3-CNF formula, in [KPY19] the authors define $\mathcal{R}_{\rho, S}^{\text {yes }}$ as the pairs $(x, w)$ where $w$ is a "locally satisfying assignment". This means that every clause $C$ in $x$ with all variables in $S$, is satisfied by $w$.
} 
Our final addition is support for arguments of knowledge transfer (AoKT) [GR19]. In a nutshell, an AoKT enables to "succinctly reuse" an AoK of the opening of some commitment $C$ for constructing another AoK for commitment $D$. That is, given an opening $w$ for $C$, it enables to give a succinct proof that $D$ opens to $g(w)$. Importantly, AoKTs can be based on falsifiable assumptions. Following [GR19], $\rho \in \mathcal{L}_{\text {par }}$ defines languages $\mathcal{L}_{\rho}^{\text {yes }}$ and $\mathcal{L}_{\rho}^{\text {no }}$, with $\mathcal{L}_{\rho}^{\text {no }}$ not necessarily the complement of $\mathcal{L}_{\rho}^{\text {yes }}$ (i.e. a promise problem), with their corresponding relations $\mathcal{R}_{\rho}^{\text {yes }}$ and $\mathcal{R}_{\rho}^{\text {no }}$. For no instances, the adversary provides a promise $w^{*}$ for $x$. In [GR19] $x=(C, D)$ and $\left(C, D, w^{*}\right) \in \mathcal{L}_{\rho}^{\text {no }}$ if $w^{*}$ is an opening for $C$ but $g\left(w^{*}\right)$ is not an opening for $D$. In our instantiations $x$ will be two SSB commitments to $C_{1}, \ldots, C_{d}$ and $D_{1}, \ldots, D_{d}$ such that $C_{i}$ opens to $w$ and $D_{i}$ to $g_{i}(w)$. From the two SSB commitments we can extract $C_{S}$ and $D_{S}$. Furthermore, $C_{i}$ and $D_{i}$ might not be extractable (actually, they will be Pedersen commitments) an hence the extractor can only compute $f(w, S)=\left\{\operatorname{Com}\left(c k_{i}, w\right): i \in S\right\}$.

We define the yes and no languages as

$$
\mathcal{L}_{\rho}^{\text {yes }}=\left\{x \mid \exists w \text { s.t. }(x, w) \in \mathcal{R}_{\rho, S}^{\text {yes }}\right\}, \quad \mathcal{L}_{\rho}^{\text {no }}=\left\{\left(x, w^{*}\right) \mid \exists y \text { s.t. }\left(x, y, w^{*}\right) \in \mathcal{R}_{\rho, S}^{\text {no }}\right\},
$$

where $w^{*}$ is the promise of the adversary and $y$ is the local $f$-witness that we can extract from the adversary. Intuitively, the two witnesses of the languages are different kind of objects. Witness $y$ is the value we extract from the adversary, which can't be equal to $f(w, S)$ for successful adversaries, but should lie the image of $f$ anyway. On the other hand $w$ is a "proper" witness from which an $y$ can be computed and hence belongs to the preimage of $f .{ }^{15}$

\subsection{Arguments with No-signaling extraction and Oblivious CRS Generation}

Similarly to the way we treated commitment schemes, we don't directly prove the existence of no-signaling extractors but first show the existence of an Oblivious CRS Generation algorithm. We then show the latter notion implies the former. For convenience, we start defining a quasi argument without no-signaling extraction but only local soundness. For local soundness, we use a weaker variant of the strong Quasi-Adaptive soundness of [JR13] where the adversary chooses $(\rho, \theta) \in \mathcal{L}_{\text {par }}$. Instead, we honestly sample parameter $\rho$ and reveal part of the witness $h_{l s}(\theta)$ to the adversary, for some function $h_{l s}$. When we don't require computational assumptions on $\rho$, as in quasi arguments of membership in a linear space, $h_{l s}$ might be the identity function and then our definition becomes strong soundness as defined in [JR13]. In knowledge transfer arguments, soundness holds provided the hardness of some computational assumption defined by $\rho$. For this reason $h_{l s}$ can't be the identity and some part of $\theta$ must remain hidden.

In practice $h_{l s}$ models correlated information leaked by another protocol, typically as a result of sharing the commitment keys. If local knowledge soundness holds even when the adversary is given $h_{l s}(\theta)$, it means that any other protocol for which the crs can be derived from $h_{l s}(\theta)$ can be safely executed with a "correlated crs".

It will be useful to consider vectors of sets of size $t$. Namely $S=\left(S_{1}, \ldots, S_{t}\right)$, for some $t \in \mathbb{N}$.

Definition 7. An $h_{l s}$-strong locally extractable proof system $\Pi$ for the parameter language $\mathcal{L}_{\text {par }}$ and relations $\mathcal{R}_{\rho}^{\text {yes }}, \mathcal{R}_{\rho, S}^{\text {no }}$ is a tuple of PPT algorithms $\Pi=(\mathrm{K}$, Prove, Verify, Extract $)$ where

- $(\rho, \theta) \leftarrow \mathcal{D}_{\text {par }}(g k, d, K, S)$ : Parameter generation $\mathcal{D}_{\text {par }}$ takes as input a group key $g k$, the locality parameter $K$ and a set $S \subseteq([d], \ldots,[d])$ with $|S| \leq K$; it outputs an instance witness pair $(\rho, \theta)$ of $\mathcal{L}_{\text {par. }}$.

${ }^{15}$ The original definition from [GR19] is syntactically different as $w$ is part of the statement in the yes language. However, as the authors said, the verifier can't read $w$ as it will render the verification process not succinct. Since $y$ becomes irrelevant, we prefer to eliminate it from the yes language. 
- $(\mathrm{crs}, \tau) \leftarrow \mathrm{K}(\rho, \theta)$ : $\mathrm{K}$ takes as input an instance-witness pair $(\rho, \theta)$ of $\mathcal{L}_{\text {par }}$; it outputs a common reference string crs and an extraction trapdoor $\tau$.

- $\pi \leftarrow \operatorname{Prove}(\operatorname{crs}, x, w)$ : Prove takes as input crs and a statement-witness pair $(x, w)$ of $\mathcal{L}_{\rho}^{\text {yes }}$; it outputs a proof $\pi$.

- $b \leftarrow \operatorname{Verify}(\operatorname{crs}, x, \pi)$ : Verify takes as input crs, a statement $x$ and a proof $\pi$; it outputs a bit $b$ indicating if the proof $\pi$ is a valid proof.

- $y \leftarrow \operatorname{Extract}(\tau, x, \pi)$ : Extract takes as input the extraction trapdoor $\tau$, a statement $x$ and a proof $\pi$, and outputs a local witness $y$ for the set $S$.

For all $\kappa \in \mathbb{N}^{t}, \boldsymbol{K} \leq(d, \ldots, d) \in \mathbb{N}^{t}, S \subseteq([d], \ldots,[d])$, with $|S| \leq \boldsymbol{K}, \Pi$ satisfies the following properties:

- Completeness: For all $(\rho, \theta) \in \mathcal{L}_{\text {par }}$ and $x, w \in\{0,1\}^{*}$

$$
\operatorname{Pr}_{g k \leftarrow \mathcal{G}\left(1^{\kappa}\right)}\left[\begin{array}{c|c}
\operatorname{Verify}(\operatorname{crs}, x, \pi)=1 & (\operatorname{crs}, \tau) \leftarrow \mathrm{K}(\rho, \theta) ; \\
\vee(x, w) \notin \mathcal{R}_{\rho, S}^{\mathrm{yes}} & \pi \leftarrow \operatorname{Prove}(\operatorname{crs}, x, w)
\end{array}\right] \geq 1-\operatorname{negl}(\kappa)
$$

- $h_{l s}$-Strong Local Knowledge Soundness: For all PPT $\mathcal{A}$

$$
\operatorname{Pr}_{g k \leftarrow \mathcal{G}\left(1^{\kappa}\right)}\left[\begin{array}{c|r}
(\rho, \theta) & \leftarrow \mathcal{D}_{\text {par }}(g k, d, K, S) ; \\
\operatorname{Verify}(\operatorname{crs}, x, \pi)=0 & (\operatorname{crs}, \tau) \leftarrow \mathrm{K}(\rho, \theta) ; \\
\vee\left(x, y, w^{*}\right) \notin \mathcal{R}_{\rho, S}^{\text {no }} & \left(x, w^{*}, \pi\right) \leftarrow \mathcal{A}\left(\rho, h_{l s}(\theta), \operatorname{crs}\right) ; \\
& y \leftarrow \operatorname{Extract}(\tau, x, \pi)
\end{array}\right] \geq 1-\operatorname{negl}(\kappa)
$$

Next, we define the no-signaling property of quasi-arguments. Similarly as with strong knowledge soundness, we consider a stronger definition where the adversary is given some function of $\theta$, namely $h_{n s}(\theta)$.

Definition 8. An $h_{l s}$-strong locally extractable proof system $\Pi$ for the parameter language $\mathcal{L}_{\text {par }}$ and relations $\mathcal{R}_{\rho, S}^{\text {yes }}, \mathcal{R}_{\rho, S}^{\text {no }}$ is an $\left(h_{l s}, h_{n s}\right)$-quasi argument if it satisfies $h_{n s}$-strong no-signaling extraction. That is, for all $\kappa \in \mathbb{N}, K \leq d \in \mathbb{N}^{t}, S^{\prime} \subseteq S \subseteq([d], \ldots,[d])$ with $|S| \leq K$, and all PPT $\mathcal{A}$ and PPT $\mathcal{D}$

$$
\begin{aligned}
& \operatorname{Pr}_{g k \leftarrow \mathcal{G}\left(1^{\kappa}\right)}\left[\mathcal{D}\left(\text { crs }, x, \pi, y_{s^{\prime}}\right)=1 \mid \begin{array}{r}
(\rho, \theta) \leftarrow \mathcal{D}_{\mathrm{par}}(g k, d, \boldsymbol{K}, \boldsymbol{S}) ; \\
(\mathrm{crs}, \tau) \leftarrow \mathrm{K}(\rho, \theta) ; \\
(x, \pi) \leftarrow \mathcal{A}\left(\rho, h_{n s}(\theta), \mathrm{crs}\right) ; \\
\text { if Verify }(\mathrm{crs}, x, \pi)=0: \operatorname{set} x=\perp ; \\
y \leftarrow \operatorname{Extract}(\tau, x, \pi)
\end{array}\right]- \\
& \operatorname{Pr}_{g k \leftarrow \mathcal{G}\left(1^{\kappa}\right)}\left[\mathcal{D}\left(\operatorname{crs}, x, \pi, y^{\prime}\right)=1 \mid \begin{array}{r}
(\rho, \theta) \leftarrow \mathcal{D}_{\mathrm{par}}\left(g k, d, \boldsymbol{K}, \boldsymbol{S}^{\prime}\right) ; \\
(\mathrm{crs}, \tau) \leftarrow \mathrm{K}(\rho, \theta) ; \\
(x, \pi) \leftarrow \mathcal{A}\left(\rho, h_{n s}(\theta), \mathrm{crs}\right) ; \\
\text { if Verify }(\mathrm{crs}, x, \pi)=0: \operatorname{set} x=\perp ; \\
y^{\prime} \leftarrow \operatorname{Extract}(\tau, x, \pi)
\end{array}\right] \mid \leq \operatorname{negl}(\kappa)
\end{aligned}
$$

Finally, we define the notion of oblivious locally extractable proof systems. The requirements are that (1) the crs alone does not help PPT adversaries gain information about the extraction set used to sample the parameters $\rho$; $(2)$ there exists a PPT algorithm OblSetup that on input a set $S^{\prime} \subseteq S$ and $(\rho, \mathrm{crs})$, sampled for extraction on the superset of $S$, outputs new 
values $\left(\rho^{\prime}, \mathrm{crs}^{\prime}\right)$ that are statistically close to $(\rho, \mathrm{crs})$ and additionally, it outputs a trapdoor $\tau^{\prime}$ for $S^{\prime}$ that outputs indistinguishable witnesses to the ones output for $S$ and restricted to $S^{\prime}$.

We consider also a " $h_{n s}$-strong" variant of (1). Note that (2) holds against unbounded adversaries which can compute $\theta$ by themselves.

Definition 9. A locally extractable proof system $\Pi$ for the parameter language $\mathcal{L}_{\text {par }}$ and relations $\mathcal{R}_{\rho}^{\text {yes }}, \mathcal{R}_{\rho, S}^{\text {no }}$ is $h_{n s}$-Strong Oblivious if there exist a PPT algorithm OblSetup such that, for all $\kappa \in \mathbb{N}, K \leq(d, \ldots, d) \in \mathbb{N}^{t}, \boldsymbol{S}^{\prime}, S \subseteq([d], \ldots,[d])$ with $\left|S^{\prime}\right|,|S| \leq K$,

1. $h_{n s}$-Strong Index Set Hiding: for all PPT $\mathcal{D}$

$$
\begin{aligned}
& \mid \operatorname{Pr}_{g k \leftarrow \mathcal{G}\left(1^{\kappa}\right)}\left[\mathcal{D}\left(\rho, \mathrm{crs}, h_{n s}(\theta)\right) \mid \begin{array}{r}
(\rho, \theta) \leftarrow \mathcal{D}_{\text {par }}(g k, d, K, S) \\
(\text { crs }, \tau) \leftarrow \mathrm{K}(\rho, \theta)
\end{array}\right]-
\end{aligned}
$$

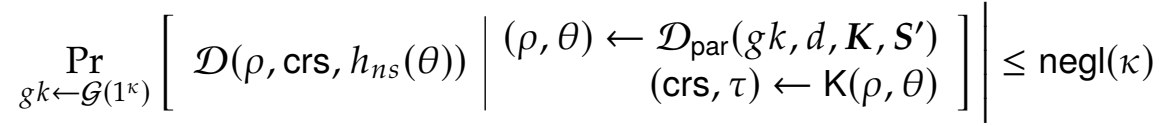

2. Oblivious trapdoor Generation: if $S^{\prime} \subseteq S$ then for all, (even unbounded) adversaries $\mathcal{A}$ and distinguishers $\mathcal{D}$

$$
\begin{aligned}
& \operatorname{Pr}_{g k \leftarrow \mathcal{G}\left(1^{\kappa}\right)}\left[\mathcal{D}\left(\rho^{\prime}, \mathrm{crs}^{\prime}, y^{\prime}\right)=1 \mid \begin{array}{r}
(\rho, \theta) \leftarrow \mathcal{D}_{\text {par }}(g k, d, K, S) ;(\operatorname{crs}, \tau) \leftarrow \mathrm{K}(\rho, \theta) \\
\left(\rho^{\prime}, \operatorname{crs}^{\prime}, \tau^{\prime}\right) \leftarrow \operatorname{OblSetup}\left(\rho, \operatorname{crs}, \boldsymbol{S}^{\prime}\right) \\
(x, \pi) \leftarrow \mathcal{A}\left(\rho, \mathrm{crs}^{\prime}\right) \\
\text { if Verify }(\operatorname{crs}, x, \pi)=0: \operatorname{set} x=\perp ; \\
y^{\prime} \leftarrow \operatorname{Extract}\left(\tau^{\prime}, x, \pi\right)
\end{array}\right]- \\
& \operatorname{Pr}_{g k \leftarrow \mathcal{G}\left(1^{\kappa}\right)}\left[\mathcal{D}\left(\rho, \mathrm{crs}, y_{S^{\prime}}\right)=1 \mid \begin{array}{r}
(\rho, \theta) \leftarrow \mathcal{D}_{\text {par }}(g k, d, K, S) ;(\operatorname{crs}, \tau) \leftarrow \mathrm{K}(\rho, \theta) \\
(x, \pi) \leftarrow \mathcal{A}(\rho, \mathrm{crs}) \\
\text { if } \operatorname{Verify}(\mathrm{crs}, x, \pi)=0: \operatorname{set} x=\perp ; \\
y \leftarrow \operatorname{Extract}(\tau, x, \pi)
\end{array}\right] \mid \leq \operatorname{negl}(\kappa)
\end{aligned}
$$

Next, we present a proof that if a locally extractable proof system satisfies oblivious crs generation, then it is no-signaling. The proof is similar to the proof of Thm. 2.

Theorem 7. Let $\Pi=\left(\mathrm{K}\right.$, Prove, Verify, Extract, OblSetup) be an $h_{n s}$-strong Locally Extractable Proof System for the parameter language $\mathcal{L}_{\mathrm{par}}$ and relations $\mathcal{R}_{\rho}^{\mathrm{yes}}, \mathcal{R}_{\rho, S}^{\mathrm{no}}$. Then, $\Pi$ has $h_{n s^{-s t r o n g}}$ no signaling extraction.

Proof. Fix any $S^{\prime} \subseteq S \subseteq([d], \ldots,[d])$ with $|S| \leq K$, and let $\mathcal{D}$ be a PPT distinguisher against no signaling extraction for these values, on instance-proof pairs output by a PPT $\mathcal{A}$. We show by a sequence of games that its success probability is negligible.

$\operatorname{Game}_{0}^{\mathcal{D}, \mathcal{A}}\left(1^{\kappa}\right)$ : We execute $(\rho, \theta) \leftarrow \mathcal{D}_{\text {par }}(g k, d, K, S) ;(\operatorname{crs}, \tau) \leftarrow \mathrm{K}(\rho, \theta)$; we then get $(x, \pi) \leftarrow \mathcal{A}\left(\rho, \mathrm{crs}, h_{n s}(\theta)\right)$ and change $x$ to $\perp$ if $\operatorname{Verify}(\operatorname{crs}, x, \pi)=0$; we compute $y \leftarrow$ $\operatorname{Extract}(\tau, x, \pi)$. The output is $\mathcal{D}\left(\mathrm{crs}, x, \pi, y_{S^{\prime}}\right)$.

$\operatorname{Game}_{1}^{\mathcal{D}, \mathcal{A}}\left(1^{\kappa}\right)$ : We execute $(\rho, \theta) \leftarrow \mathcal{D}_{\text {par }}(g k, d, K, S)$; (crs, $\left.\tau\right) \leftarrow \mathrm{K}(\rho, \theta)$; we use the oblivious extractor to get $\left(\rho^{\prime}, \operatorname{crs}^{\prime}, \tau^{\prime}\right) \leftarrow \operatorname{OblSetup}\left(\rho, \operatorname{crs}, S^{\prime}\right)$; we then get $(x, \pi) \leftarrow \mathcal{A}\left(\rho^{\prime}, \operatorname{crs}^{\prime}, h_{n s}(\theta)\right)$ and change $x$ to $\perp$ if $\operatorname{Verify}(\operatorname{crs}, x, \pi)=0$; we compute $y^{\prime} \leftarrow \operatorname{Extract}\left(\tau^{\prime}, x, \pi\right)$. The output is $\mathcal{D}\left(\operatorname{crs}, x, \pi, y^{\prime}\right)$. 
$\operatorname{Game}_{2}^{\mathcal{D}, \mathcal{A}}\left(1^{\kappa}\right)$ : This is the same as Game ${ }_{1}^{\mathcal{D}, \mathcal{A}}$ but in the first step we sample parameters for $S^{\prime}$, that is we execute $(\rho, \theta) \leftarrow \mathcal{D}_{\text {par }}\left(g k, d, K, S^{\prime}\right)$.

$\operatorname{Game}_{3}^{\mathcal{D}, \mathcal{A}}\left(1^{\kappa}\right)$ : We execute $(\rho, \theta) \leftarrow \mathcal{D}_{\text {par }}\left(g k, d, K, S^{\prime}\right)$; $(\mathrm{crs}, \tau) \leftarrow \mathrm{K}(\rho, \theta)$; we then get $(x, \pi) \leftarrow \mathcal{A}\left(\rho, \mathrm{crs}, h_{n s}(\theta)\right)$ and change $x$ to $\perp$ if Verify $(\mathrm{crs}, x, \pi)=0$; we compute $y^{\prime} \leftarrow$ $\operatorname{Extract}(\tau, x, \pi)$. The output is $\mathcal{D}\left(\mathrm{crs}, x, \pi, y^{\prime}\right)$.

We next show that for all $1 \leq i \leq 3$,

$$
\left|\operatorname{Pr}\left[\operatorname{Game}_{i}^{\mathcal{D}, \mathcal{A}}\left(1^{\kappa}\right)=1\right]-\operatorname{Pr}\left[\operatorname{Game}_{i-1}^{\mathcal{D}, \mathcal{A}}\left(1^{\kappa}\right)=1\right]\right| \leq \operatorname{negl}(\kappa) .
$$

- Case $i=1, i=3$. Note that for $i=1$, the difference in the two games is exactly as in the two cases of the oblivious trapdoor generation property for $S^{\prime} \subseteq S$, so the outputs of games are statistically close. For case 3, we use the same argument for $S^{\prime} \subseteq S^{\prime}$.

- Case $i=2$ The only difference in the games is how we setup the initial crs, either by sampling for $S^{\prime}$ or for $S$. The output of the two games are computationally indistinguishable by the index set hiding property, even when the adversary is given $h_{n s}(\theta)$.

By a standard argument we get that, for all PPT $\mathcal{D}, \mathcal{A}$,

$$
\left|\operatorname{Pr}\left[\operatorname{Game}_{0}^{\mathcal{D}, \mathcal{A}}\left(1^{\kappa}\right)=1\right]-\operatorname{Pr}\left[\operatorname{Game}_{5}^{\mathcal{D}, \mathcal{A}}\left(1^{\kappa}\right)=1\right]\right| \leq \operatorname{negl}(\kappa) .
$$

Finally, noting that $\mathrm{Game}_{0}^{\mathcal{D}, \mathcal{A}}, \mathrm{Game}_{3}^{\mathcal{D}, \mathcal{A}}$ correspond to the two cases of no signaling extraction, we conclude the proof.

\subsection{Succinct Pairing Based Quasi-Arguments}

In this section we present quasi arguments for various languages using SSB commitments with oblivious trapdoor generation. We first present the simpler case, membership in linear spaces, and then we present some extensionsof it, specifically a knowledge transfer version, and a knowledge transfer version for statements split in the two groups. Finally,we use the latter to build a quasi argument of knowledge transfer for hadamard products.

\subsubsection{Quasi Arguments of Membership in Linear Spaces}

Let $\mathcal{U}$ be a witness samplable distributions sampling $\left([\mathbf{U}]_{1}, \mathbf{U}\right)$, where $\mathbf{U} \in \mathbb{Z}_{p}^{d \times n}$. We assume that for any $S \subseteq[d]$, given only $\left[\mathbf{U}_{S}\right]_{1}$ such that $\mathbf{U}=\mathbf{P}_{S}^{\top}\left(\begin{array}{l}\mathbf{U}_{S} \\ \mathbf{U}_{\bar{S}}\end{array}\right)$ there is an efficient way of sampling $\left[\mathbf{U}_{\bar{S}}\right] \cdot{ }^{16}$ Also, let CS be an algebraic SSB commitment key. The parameter language is

$$
\begin{aligned}
\mathcal{L}_{\text {par }}=\left\{[\mathbf{U}]_{1},[\mathbf{G}]_{1} \mid\right. & \exists \mathbf{U}, \mathbf{G} \text { s.t. }\left([\mathbf{U}]_{1}, \mathbf{U}\right) \in \operatorname{Sup}(\mathcal{U}) \text { and } \\
& \left.\left([\mathbf{G}]_{1}, \mathbf{G}, \mathbf{T}\right) \in \operatorname{Sup}(\operatorname{CS} . \operatorname{KeyGen}(g k, d, K, S))\right\}
\end{aligned}
$$

We assume that the corresponding relation is efficiently verifiable ${ }^{17}$. The parameters $\rho=$ $\left([\mathbf{U}]_{1},[\mathbf{G}]_{1}\right) \leftarrow(\mathcal{U}$, CS.KeyGen $(g k, d, K, S))$ define the following relations:

$$
\begin{aligned}
& \mathcal{R} \mathcal{L}_{\rho}^{\text {yes }}=\left\{\left([\boldsymbol{c}]_{1}, \boldsymbol{w}\right): \boldsymbol{c}=\mathbf{G U} \boldsymbol{w}\right\}, \\
& \mathcal{R} \mathcal{L}_{\rho, S}^{\text {no }}=\left\{\left([\boldsymbol{c}]_{1},[\boldsymbol{y}]_{1}\right): \boldsymbol{y} \text { is a valid } S \text {-opening of } \boldsymbol{c} \text { and } \boldsymbol{y} \notin \operatorname{Im}\left(\mathbf{U}_{S}\right)\right\}
\end{aligned}
$$

\footnotetext{
${ }^{16}$ We will instantiate the argument with $\mathbf{U}$ a block lower triangular matrix where each row is of the form $\left(\mathbf{U}_{1}, \mathbf{U}_{2}, \ldots, \mathbf{U}_{i}, \mathbf{0}, \ldots, \mathbf{0}\right)$ where $\left\{\mathbf{U}_{i}\right\}_{i}$ are independent random variables. Then is clear that from $\left[\mathbf{U}_{S}\right]_{1}$ we know $\left[\mathbf{U}_{i}\right]_{1}$ up to $i=\max S$, and the rest $\left\{\mathbf{U}_{j}: j \notin S\right\}$ can be sampled independently.

${ }^{17}$ This is w.l.o.g. since one can extend the witness to include the randomness used to sample the parameters.
} 
The advice is the empty string while the extractor should retrieve $f(\boldsymbol{w}, S)=\left[\mathbf{U}_{S}\right]_{1} \boldsymbol{w}$ from any accepting statement and proof pair. We present the construction QALin in Fig. 4. The construction is essentially the quasi adaptive construction of membership in linear space of [KW15] for the matrix GU.

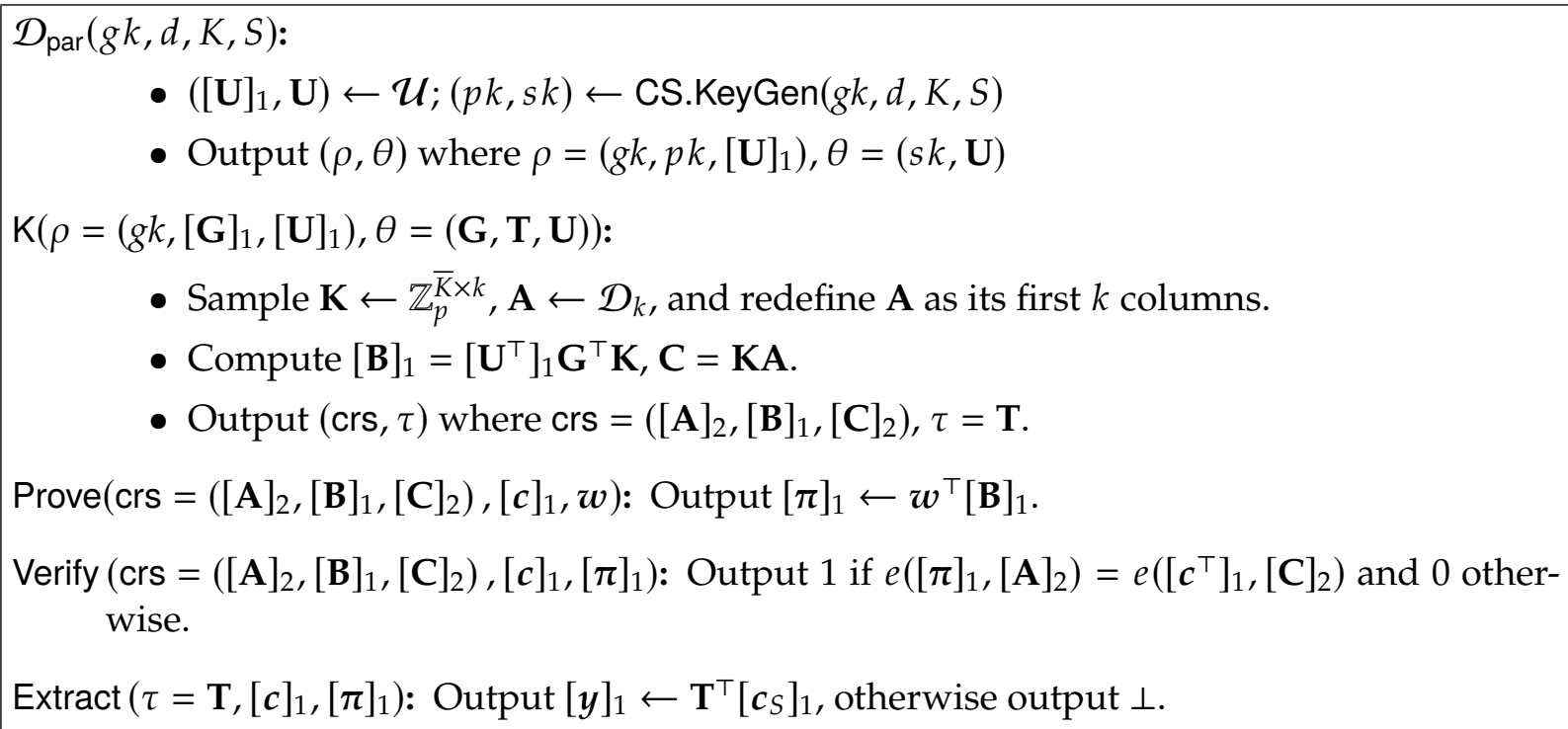

Figure 4: Construction QALin for membership in linear spaces. Note that this is just the argument of $[\mathrm{KW} 15]$ for matrix $[\mathrm{GU}]_{1}$.

Theorem 8. Let $\mathcal{U}$ be a witness samplable distribution, $\mathcal{D}_{k}$ be a matrix distribution and $\mathrm{CS}$ an algebraic SSB commitment. Then, construction QALin of Fig. 4 is a locally extractable proof system with $h_{1 \mathrm{~s}}$-strong local knowledge soundness where $h_{\mathrm{Is}}(\theta)=\theta$. Furthermore, completeness holds with probability 1 and $h_{\mathrm{ls}}$-strong local knowledge soundness holds with probability at least $1-\operatorname{Adv}_{\mathrm{snd}}^{\Pi_{\text {lin }}}(\mathcal{B})$, whee $\mathcal{B}$ is a PPT adversary against the strong soundness of $\Pi_{\text {lin }}$ of [KW15].

Proof. For completeness, we have that if $c=\mathbf{G U w}$, then

$$
c^{\top} \mathrm{C}=(\mathrm{GUw})^{\top} \mathrm{C}=\boldsymbol{w}^{\top} \mathbf{U}^{\top} \mathrm{G}^{\top} \mathrm{C}=\boldsymbol{w}^{\top} \mathbf{U}^{\top} \mathrm{G}^{\top} \mathrm{KA}=\boldsymbol{w}^{\top} \mathrm{BA}=\pi \mathrm{A} .
$$

Local knowledge soundness is guaranteed by the local extractability of the SSB commitment scheme and soundness of Kiltz and Wee proof system. Note that the extractor always outputs a valid partial opening of $[c]_{1}$ given an accepting proof $[\pi]_{1}$, by the local extractability property of the SSB commitments. We claim that this opening must lie in $\operatorname{Im}\left(\left[\mathbf{U}_{S}\right]_{1}\right)$. Assume otherwise, and let $\mathcal{A}$ be a PPT adversary that makes the extraction fail. We construct a PPT adversary $\mathcal{B}_{S}$ that breaks strong soundness of Kiltz and Wee for the matrix $\mathbf{U}_{S}$, conditioned on $\mathcal{A}$ giving a valid proof. $\mathcal{B}_{S}$ works as follows: it takes input $\mathrm{crs}_{S}$ containing $\left[\mathbf{U}_{S}\right]_{1} \in \mathbb{G}^{|S| \times d},[\mathbf{A}]_{2} \in \mathbb{G}_{2}^{k \times k}$, $\left[\mathbf{B}^{\dagger}\right]_{1} \in \mathbb{G}^{d \times k},\left[\mathbf{C}^{\dagger}\right]_{2} \in \mathbb{G}_{2}^{|S| \times k}$ and the discrete logarithms of matrix $\mathbf{U}_{S}$ and does the following:

- It samples $\left(\left[\mathbf{U}_{\bar{S}}\right]_{1}, \mathbf{U}_{\bar{S}}\right)$ s.t. $\mathbf{U}=\mathbf{P}_{S}^{\top}\left(\mathbf{U}_{S} / \mathbf{U}_{\bar{S}}\right)$.

- It samples $\left([\mathbf{G}]_{1}, \mathbf{G}, \mathbf{T}\right) \leftarrow$ CS.KeyGen $(g k, n, d, K, S)$ and a random matrix $\mathbf{R} \leftarrow \mathbb{Z}_{p}^{K+k \times k}$.

- It computes $[\mathbf{B}]_{1}=\left[\mathbf{B}^{\dagger}\right]_{1}+[\mathbf{U}]^{\top} \mathbf{G}^{\top} \mathbf{R},[\mathbf{C}]_{2}=\mathbf{T}\left[\mathbf{C}^{\dagger}\right]_{2}+\mathbf{R}[\mathbf{A}]_{2}$.

- It sets $\rho:=\left(g k,[\mathbf{G}]_{1},[\mathbf{U}]_{1}\right), \theta:=(\mathbf{G}, \mathbf{U}, \mathbf{T})$ and $\mathrm{crs}:=\left([\mathbf{A}]_{2},[\mathbf{B}]_{1},[\mathbf{C}]_{2}\right)$. 
It then executes $\mathcal{A}(\rho, \theta, \mathrm{crs})$ until it outputs $[\boldsymbol{c}]_{1},[\pi]_{1}$. If this is an accepting proof pair, $\mathcal{B}_{S}$ sets $\left[x^{\dagger}\right]:=\mathbf{T}[c]$ and $\left[\pi^{\dagger}\right]:=[\pi]_{1}-[c]_{1}^{\top} \mathbf{R}$.

First, we claim that the values $\rho, \theta$, crs given as input to $\mathcal{A}$ are identically distributed to honestly created ones and thus do not skew the probability that $\mathcal{A}$ outputs a valid proof. This is immediate for $\rho, \theta$ since they are sampled honestly. We show that this is true for crs as well.

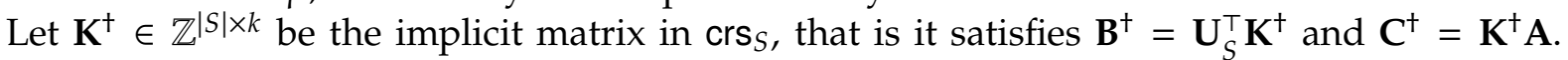
Consider the matrix $\mathbf{K}=\mathbf{T} \mathbf{K}^{\dagger}+\mathbf{R}$, and note that this matrix is uniformly distributed since $\mathbf{R}$ is uniformly distributed. Thus $\mathbf{K}$ is distributed identically to an honestly generated $\mathbf{K}^{\prime}$ for generating a crs. We claim that the crs crs output by $\mathcal{B}_{S}$ is identically distributed to sampling this matrix and computing the other values honestly. Indeed we have that

$$
\begin{aligned}
& \mathbf{C}=\mathbf{T C}^{\dagger}+\mathbf{R A} \quad \text { and } \quad \mathbf{B}=\mathbf{B}^{\dagger}+\mathbf{U}^{\top} \mathbf{G}^{\top} \mathbf{R}=\mathbf{U}_{S}^{\top} \mathbf{K}^{\dagger}+\mathbf{U}^{\top} \mathbf{G}^{\top} \mathbf{R} \\
& =\mathbf{T K}^{\dagger} \mathbf{A}+\mathbf{R A} \quad=\mathbf{U}^{\top} \mathbf{G}^{\top} \mathbf{T} \mathbf{K}^{\dagger}+\mathbf{U}^{\top} \mathbf{G}^{\top} \mathbf{R} \\
& =\left(\mathbf{T K}^{\dagger}+\mathbf{R}\right) \mathbf{A} \quad=\mathbf{U}^{\top} \mathbf{G}^{\top}\left(\mathbf{T} \mathbf{K}^{\dagger}+\mathbf{R}\right)=(\mathbf{G U})^{\top} \mathbf{K} \\
& =\mathbf{K A}
\end{aligned}
$$

where the second equality for $\mathbf{B}$ follows since by the properties of algebraic SSB commitments we have $\mathbf{T}^{\top} \mathbf{G}=\left(\mathbf{I}_{|S|} \mathbf{0}\right) \mathbf{P}_{S}$ which gives

$$
\mathbf{U}^{\top} \mathbf{G}^{\top} \mathbf{T}=\mathbf{U}^{\top} \mathbf{P}_{S}^{\top}\left(\begin{array}{c}
\mathbf{I}_{|S|} \\
\mathbf{0}
\end{array}\right)=\mathbf{U}_{S}
$$

So, the outputted crs crs' is indeed identically distributed with an honest one.

Finally, we show that if $\mathcal{A}$ outputs a valid proof $[\pi]_{1}$, then $\mathcal{B}_{S}$ outputs a valid statementproof pair w.r.t. to $\mathrm{crs}_{S}$. Indeed, by the local extractability property of the commitment scheme, $\mathcal{B}_{S}$ always outputs some $\left[x^{\dagger}\right]_{1}$ consistent with $[c]_{1}$, and also the proof verifies, since we have

$$
\pi \mathbf{A}=c^{\top} \mathbf{C}=c^{\top} \mathbf{K} \mathbf{A}=c^{\top}\left(\mathbf{T} \mathbf{K}^{\dagger}+\mathbf{R}\right) \mathbf{A}=\left(x^{\dagger}\right)^{\top} \mathbf{K}^{\dagger} \mathbf{A}+c^{\top} \mathbf{R} \mathbf{A}
$$

which gives $\pi^{\dagger} \mathbf{A}=\boldsymbol{\pi} \mathbf{A}-c^{\top} \mathbf{R} \mathbf{A}=\left(x^{\dagger}\right)^{\top} \mathbf{K}^{\dagger} \mathbf{A}=\left(x^{\dagger}\right) \mathbf{C}^{\dagger}$. We conclude that $\left[\pi^{\dagger}\right]_{1}$ is a valid proof for $\left[x^{\dagger}\right]_{1} \notin \operatorname{Im}\left(\left[\mathbf{U}_{S}\right]_{1}\right)$ and $\mathcal{B}_{S}$ breaks soundness of Kiltz and Wee construction.

Corollary 2. Consider construction from Fig. 4 with a statement of the form $\left(\begin{array}{l}{[x]_{1}} \\ {[y]_{1}}\end{array}\right)$, matrix $\left(\begin{array}{l}\mathbf{U} \\ \mathbf{V}\end{array}\right)$, locality parameter $\mathbf{L} \leq(d, d) \in \mathbb{N}^{2}$ and extraction set $S=\left(S_{1}, S_{2}\right) \subseteq([d],[d]),|S| \leq \boldsymbol{L}$, such that the $\left(\mathbf{U}_{S_{1}}^{\top}, h\right)-M D D H$ assumption is hard for some function $h$. Assume also $\mathbf{K} \leftarrow \mathbb{Z}_{p}^{L_{1}+L_{2}+2 k \times k}, \mathbf{G}=\left(\begin{array}{cc}\mathbf{G}_{1} & 0 \\ \mathbf{0} & \mathbf{G}_{2}\end{array}\right)$, where $\mathbf{G}_{i} \leftarrow$ CS.KeyGen $\left(g k, d, L_{i}, S_{i}\right)$, and $\mathbf{A} \leftarrow \mathbb{Z}_{p}^{k \times k}, k \geq 2$. Then construction from Fig. 4 is also a quasi argument for the relations $\mathcal{K} \mathcal{L}_{\rho}^{\text {yes }}=\mathcal{R} \mathcal{L}_{\rho}^{\text {yes }}$ and $\mathcal{K} \mathcal{L}_{\rho}^{\text {no }}=\left\{[\boldsymbol{c}]_{1},[d]_{1},\left[x^{*}\right]_{1},\left[\boldsymbol{y}^{*}\right]_{1}, \boldsymbol{w}^{*}\right.$ : $\left(\begin{array}{l}c \\ d\end{array}\right)$ S-open to $\left(\begin{array}{l}x^{*} \\ y^{*}\end{array}\right)$ and $x^{*}=\mathbf{U}_{S_{1}} \boldsymbol{w}^{*}$ but $\left.\boldsymbol{y}^{*} \neq \mathbf{V}_{S_{2}} \boldsymbol{w}^{*}\right\}$, with $h_{\mathrm{ls}}$-strong local soundness where $h_{\mathrm{ls}}(\theta)=$ $\left(h\left(\mathbf{U}_{S_{1}}^{\top}\right), \mathbf{G}, \mathbf{U}_{S_{2}}^{\top}\right)$.

Proof. In [GR19] it is shown that Kiltz and Wee argument is also a knowledge transfer argument whenever the $\mathbf{U}^{\top}-\mathrm{MDDH}$ assumption $\left(\mathbf{U}_{S_{1}}^{\top}-\mathrm{MDDH}\right.$ in this case) holds and $\mathbf{A}$ is not full rank. Of course, this is still true if the stronger $\left(\mathbf{U}_{S}^{\top}, h\right)$-MDDH assumption holds. However in construction of fig. $4 \mathbf{A}$ is full rank with overwhelming probability. Nevertheless, if $\mathbf{A}$ is uniform and $k \geq 2$ we can jump to a game (relying on the DDH assumption) where $\mathbf{A} \in \mathbb{Z}_{p}^{k \times k}$ is not full rank. Then the reduction of Thm. 8 yields also a reduction to the knowledge transfer of [KW15] (taking $\left(\begin{array}{l}\mathbf{T}_{1} \\ \mathbf{T}_{2}\end{array}\right)$ as trapdoor, where $\mathbf{T}_{i}$ is the trapdoor for $\left.\mathbf{G}_{i}\right)$. 
The proof that QALin is oblivious essentially follows from the oblivious trapdoor generation and index set hiding of SSB commitments. Before proving oblivious trapdoor generation we present a lemma stating that we can also compute $\rho$, crs knowing only the commitment key $[\mathbf{G}]_{1}$ and $\mathbf{U}$, in both simple and knowledge transfer schemes.

Lemma 1. There exists a modified crs generation algorithm $\mathrm{K}^{\prime}$ that on input $\left(\rho, \theta^{\prime}\right)$, where $\theta^{\prime}$ contains only $\mathbf{U}$ (resp. $\mathbf{U}, \mathbf{V})$ outputs a crs such that $(\rho, \mathrm{crs})$ are identically distributed to the honest algorithm.

The lemma follows directly by noting that $[\mathbf{B}]_{1}=\left[\mathbf{U}^{\top}\right]_{1} \mathbf{G K}=\mathbf{U}^{\top}[\mathbf{G}]_{1} \mathbf{K}$. (resp. $[\mathbf{B}]_{1}=\left[\mathbf{U}^{\top}\right.$ | $\left.\mathbf{V}^{\top}\right]_{1} \mathbf{G K}=\left(\mathbf{U}^{\top} \mid \mathbf{V}^{\top}\right)[\mathbf{G}]_{1} \mathbf{K}$. Given that this result holds, we slightly abuse notation and refer to $K^{\prime}\left(\rho, \theta^{\prime}\right)$ as $K\left(\rho, \theta^{\prime}\right)$, that is we use the same name for the honest and the simulated algorithm.

Theorem 9. Let $\mathcal{U}$ (resp. $\mathcal{U}, \mathcal{V}$ for the knowledge transfer case) be a witness samplable distribution, and CS be an algebraic SSB commitment scheme with perfect completeness, $h$-strong index set hiding and oblivious trapdoor generation. Then Construction QALin of Fig. 4 (resp. construction $\Pi$ of corollary 2) is $h_{\mathrm{ns}}$-strong oblivious where $h_{\mathrm{ns}}=(h(s k), \mathbf{U})\left(\right.$ resp. $\left.h_{\mathrm{ns}}=(h(s k), \mathbf{U}, \mathbf{V})\right)$. Furthermore,

1. For every PPT $\mathcal{A}$ against $h_{\mathrm{ns}}$-strong index set hiding of $\Pi$, there exists an adversary $\mathcal{B}$ against $h$-strong index set hiding property of CS, such that $\operatorname{Adv}_{\text {ISH }}^{\Pi}(\mathcal{A}) \leq \operatorname{Adv}_{\mathrm{ISH}}^{\mathrm{CS}}(\mathcal{B})$ where $h_{\mathrm{ns}}(\theta)=$ $(h(s k), \mathbf{U})$.

2. For every $\mathcal{A}$ against oblivious trapdoor generation of $\Pi$, there exists an adversary $\mathcal{B}$ against oblivious trapdoor generation of CS, such that $\operatorname{Adv}_{\text {oblv }}^{\Pi}(\mathcal{A}) \leq \operatorname{Adv}_{\text {oblv }}^{\mathrm{CS}}(\mathcal{B})$.

Proof. For index set hiding, it is enough to notice that in both cases, the crs of $\Pi$ can be efficiently computed given only $c k=\left([\mathbf{G}]_{1}, h(\mathbf{G})\right)$. Indeed by sampling $[\mathbf{U}]_{1}, \mathbf{U} \leftarrow \mathcal{U}$ (resp. $[\mathbf{U}]_{1}, \mathbf{U} \leftarrow \mathcal{U}$; $\left.[\mathbf{V}]_{1}, \mathbf{V} \leftarrow \mathcal{V}\right)$ all values of crs are efficiently computable, as noted in the previous lemma. Additionally, since we assume CS is $h$-strong ISH, $\mathcal{A}$ can be also given $h_{\mathrm{ns}}(\theta)=(h(s k), \mathbf{U})$ (resp. $\left.h_{\mathrm{ns}}(\theta)=(h(s k), \mathbf{U}, \mathbf{V})\right)$. Thus, a distinguishing advantage in index set hiding of $\Pi$ immediately implies equal advantage on the respective property of CS.

For oblivious trapdoor generation we first describe the OblSetup algorithm. Let $S^{\prime} \subseteq S$.

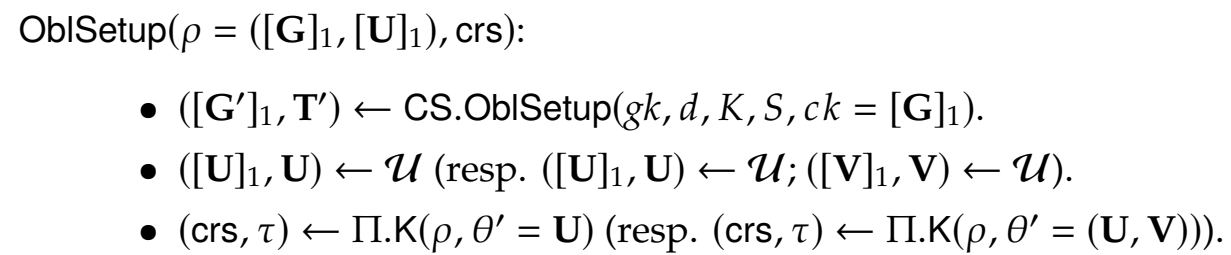

Note that the only difference in sampling with $S$ and with $S^{\prime}$ is how we sample the commitment key G. The crs part crs is identically distributed to an honest one by Lemma 1. Finally, by the statistically binding property of the commitment key the extracted witness for $S$ and $S^{\prime}$ are unique and thus do not help the (unbounded) distinguisher, who can compute them on its own.

Corollary 3. When CS is the one from fig. 3, then $\Pi$ from fig. 4 (resp. corollary 2) is $h_{\mathrm{ns}}$-strong no-signaling where $h_{\mathrm{ns}}(\theta)=(h(s k), \mathbf{U})\left(\right.$ resp. $\left.h_{\mathrm{ns}}(\theta)=(h(s k), \mathbf{U}, \mathbf{V})\right)$.

Proof. Follows directly from Theorem 7 and the $h_{n s}$-strong ISH of QALin, which in turn follows from Theorem 9. 
Extensions. We consider several extensions of QALin such as bilateral linear spaces [GHR15b], where the statement as well as the generating matrix have components in both groups. We also consider a sum argument [GHR15b] which is akin to a bilateral language but one shows that the sum of the discrete logs of two vectors in $\mathbb{G}_{1}$ and $\mathbb{G}_{2}$ belong to the image of the sum of two matrices in $\mathbb{G}_{1}, \mathbb{G}_{2}$. Finally, we extend local soundness to consider knowledge transfer arguments. The security of all this extensions is almost verbatim of theorems 8 and 9 .

Quasi Argument for Bilateral Linear Knowledge Transfer. Let $\mathcal{M}, \mathcal{N}_{1}, \mathcal{N}_{2}$ be 3 witness samplable distribution over matrices in $\mathbb{G}_{1}^{d \times n}, \mathbb{G}_{1}^{d \times n}$ and $\mathbb{G}_{2}^{d \times n}$, respectively, for $n, d \in \mathbb{N}$. Let $K \leq d$ where $K=\left(K_{1}, K_{2}\right)$ and $S \subseteq([d],[d])$ where $S=S_{1} \cup S_{2}$ and $S \leq K$ Let CS be an algebraic SSB commitment schemes with commitment space $\mathbb{G}_{\mu}^{\bar{K}}$, where $\mathbb{G}_{\mu}$ is defined by the input $g k$. The parameter language is

$$
\begin{aligned}
\mathcal{L}_{\text {par }}=\left\{[\mathbf{M}]_{1},\right. & {\left[\mathbf{N}_{1}\right]_{1},\left[\mathbf{N}_{2}\right]_{2},[\mathbf{G}]_{1},[\mathbf{H}]_{1},[\mathbf{F}]_{2} \mid \exists \mathbf{M}, \mathbf{N}_{1}, \mathbf{N}_{2}, \mathbf{G}, \mathbf{H}, \mathbf{F} \text { s.t. } } \\
& \left([\mathbf{M}]_{1}, \mathbf{M}\right),\left(\left[\mathbf{N}_{1}\right]_{1}, \mathbf{N}_{2}\right),\left(\left[\mathbf{N}_{2}\right]_{2}, \mathbf{N}_{2}\right) \in \operatorname{Sup}\left(\mathcal{M}, \mathcal{N}_{1}, \mathcal{N}_{2}\right), \\
& \left([\mathbf{G}]_{1}, \mathbf{G}, \mathbf{T}_{\mathbf{G}}\right) \in \operatorname{Sup}\left(\text { CS.KeyGen }\left(g k_{1}, d, K_{1}, S_{1}\right)\right), \\
& \left([\mathbf{H}]_{1}, \mathbf{H}, \mathbf{T}_{\mathbf{H}}\right) \in \operatorname{Sup}\left(\text { CS.KeyGen }\left(g k_{1}, d, K_{2}, S_{2}\right)\right), \\
& \left.\left([\mathbf{F}]_{2}, \mathbf{F}, \mathbf{T}_{\mathbf{F}}\right) \in \operatorname{Sup}\left(\text { CS.KeyGen }\left(g k_{2}, d, K_{2}, S_{2}\right)\right)\right\}
\end{aligned}
$$

We assume w.l.o.g. that the corresponding relation is efficiently verifiable.The parameters $\rho=\left([\mathbf{M}]_{1},\left[\mathbf{N}_{1}\right]_{1},\left[\mathbf{N}_{2}\right]_{2},[\mathbf{G}]_{1},[\mathbf{H}]_{1},[\mathbf{F}]_{2}\right)$, define the following relations:

$$
\begin{aligned}
& \mathcal{R}_{\rho}^{\mathrm{yes}}=\left\{[\boldsymbol{c}]_{1},\left[\boldsymbol{d}_{1}\right]_{1},\left[\boldsymbol{d}_{2}\right]_{2}, \boldsymbol{w} \mid\left(\begin{array}{c}
\boldsymbol{c} \\
\boldsymbol{d}_{1} \\
\boldsymbol{d}_{2}
\end{array}\right)=\left(\begin{array}{c}
\mathbf{G M} \\
\mathbf{H N} \\
\mathbf{F N}_{2}
\end{array}\right) \boldsymbol{w}\right\},
\end{aligned}
$$

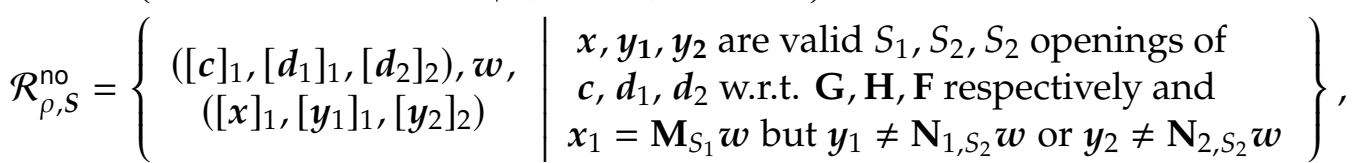

that is the partial witness for $S$ is some valid local openings $[x]_{1},\left[y_{1}\right]_{1},\left[y_{2}\right]_{2}$ w.r.t. to $\mathbf{G}, \mathbf{H}, \mathbf{F}$ respectively that satisfy the following: if $x_{S_{2}}=\mathbf{M}_{S_{1}} \boldsymbol{w}$ then it should be the case that both $y_{1}=\mathbf{N}_{1, S_{2}} \boldsymbol{w}$ and $\boldsymbol{y}_{\mathbf{2}}=\mathbf{N}_{2, S_{2}} \boldsymbol{w}$ where $\boldsymbol{w}$ is the promise of the adversary. Note that if $S_{1}$ is the empty set the latter relations trivially hold. We present the protocol in Fig. 5. Security is almost verbatim to the unilateral case.

Theorem 10. Let $\mathcal{M}, \mathcal{N}_{1}, \mathcal{N}_{2}$ be witness samplable distributions, $\mathcal{D}_{k}$ be a matrix distribution and $\mathrm{CS}$ an algebraic SSB commitment with perfect completeness. Also, let $\mathcal{A}$ be an adversary against $h_{\mathrm{ls}}$-strong local knowledge soundness of construction QABlin of Fig. 5, where the index $h_{\mathrm{ls}}(\theta)=$ $\left(\mathbf{G}, \mathbf{H}, \mathbf{F}, h\left(\mathbf{M}, \mathbf{N}_{1}, \mathbf{N}_{2}\right)\right)$. Then, completeness holds with probability 1 and $h_{1 \mathrm{~s}}$-strong local knowledge soundness holds with probability at least $1-\operatorname{Adv}_{\text {snd }}^{\Pi_{k-l i n}}\left(\mathcal{B}_{S}\right)$, where $\mathcal{B}_{S}$ is any PPT adversary against $h$-strong soundness of $\Pi_{k t-l i n}$ and $h$ giving the discrete logarithms of the last two matrices. 
Proof. For completeness, we have that

$$
\begin{aligned}
\left(c^{\top} \mid d_{1}^{\top}\right) \mathbf{C}_{1}+d_{2}^{\top} \mathbf{C}_{2} & =\left(c^{\top} \mid d_{1}^{\top}\right)\left(\begin{array}{l}
\mathbf{K}_{1} \\
\mathbf{K}_{2}
\end{array}\right) \mathbf{A}+d_{2}^{\top} \mathbf{K}_{2} \mathbf{A} \\
& =\left(c^{\top} \mathbf{K}_{1}+d_{1}^{\top} \mathbf{K}_{2}+d_{2}^{\top} \mathbf{K}_{2}\right) \mathbf{A} \\
& =\left(\boldsymbol{w}^{\top} \mathbf{M}^{\top} \mathbf{G}^{\top} \mathbf{K}_{1}+\boldsymbol{w}^{\top} \mathbf{N}_{1}^{\top} \mathbf{H}^{\top} \mathbf{K}_{2}+\boldsymbol{w}^{\top} \mathbf{N}_{2}^{\top} \mathbf{F}^{\top} \mathbf{K}_{2}\right) \mathbf{A} \\
& =\left(\boldsymbol{w}^{\top}\left(\mathbf{M}^{\top} \mathbf{G}^{\top} \mathbf{K}_{1}+\mathbf{N}_{1}^{\top} \mathbf{H}^{\top} \mathbf{K}_{2}\right)+\boldsymbol{w}^{\top} \mathbf{N}_{2}^{\top} \mathbf{F}^{\top} \mathbf{K}_{2}\right) \mathbf{A} \\
& =\boldsymbol{w}^{\top} \mathbf{B} \mathbf{A}+\boldsymbol{w}^{\top} \mathbf{D A} \\
& =\boldsymbol{\pi} \mathbf{A}+\boldsymbol{\theta} \mathbf{A}
\end{aligned}
$$

Local Extractability follows using almost an identical argument to Thm. 8 and reducing to knowledge transfer of linear KTA Argument of [GR19] presented in Fig. 1. Given an adversary $\mathcal{A}$ breaking $h_{\mathrm{ls}}$-Strong local knowledge soundness of QABlin we construct another adversary $\mathcal{B}_{S}$ that breaks $h$-strong soundness of the argument $\Pi_{k t-l i n}$ for matrices $\left[\mathbf{M}_{S_{1}}\right]_{1},\left[\mathbf{N}_{1, S_{2}}\right]_{1}$ and $\left[\mathbf{N}_{2, S_{2}}\right]_{2}$. $\mathcal{B}_{S}$ works as follows: it takes input $\left(\rho^{\dagger}, h\left(\theta^{\dagger}\right), \mathrm{crs}^{\dagger}\right)$ where

$$
\begin{gathered}
\rho^{\dagger}:=\left(g k,\left[\mathbf{M}_{S_{1}}\right]_{1},\left[\mathbf{N}_{1, S_{2}}\right]_{1},\left[\mathbf{N}_{2, S_{2}}\right]_{2}\right), \quad h\left(\theta^{\dagger}\right):=\left(\mathbf{N}_{1, S_{2}}, \mathbf{N}_{2, S_{2}}\right), \\
\operatorname{crs}^{\dagger}:=\left(\left[\mathbf{B}^{\dagger}\right]_{1},\left[\mathbf{D}^{\dagger}\right]_{2},[\mathbf{A}]_{1,2},\left[\mathbf{C}_{1}^{\dagger}\right]_{2},\left[\mathbf{C}_{2}^{\dagger}\right]_{1}\right)
\end{gathered}
$$

and does the following:

- $\left([\mathbf{G}]_{1}, \mathbf{G}, \mathbf{T}_{\mathbf{G}}\right) \leftarrow \operatorname{CS} . \operatorname{KGen}\left(g k_{1}, d, K_{1}, S_{1}\right)$.

- $\left([\mathbf{H}]_{1}, \mathbf{H}, \mathbf{T}_{\mathbf{H}}\right) \leftarrow \mathrm{CS} . \mathrm{KGen}\left(g k_{1}, d, K_{2}, S_{2}\right)$.

- $\left([\mathbf{F}]_{2}, \mathbf{F}, \mathbf{T}_{\mathbf{F}}\right) \leftarrow$ CS.KGen $\left(g k_{2}, d, K_{2}, S_{2}\right)$.

- It samples $\mathbf{M}_{\bar{S}_{1}}, \mathbf{N}_{1, \bar{S}_{2}}, \mathbf{N}_{2, \bar{S}_{2}}$, such that $\mathbf{M}=\mathbf{P}_{S_{1}}\left(\begin{array}{l}\mathbf{M}_{S_{1}} \\ \mathbf{M}_{\bar{S}_{1}}\end{array}\right), \mathbf{N}_{1}=\mathbf{P}_{S_{2}}\left(\begin{array}{l}\mathbf{N}_{1, S_{2}} \\ \mathbf{N}_{1, \bar{S}_{2}}\end{array}\right), \mathbf{N}_{2}=\mathbf{P}_{S_{2}}\left(\begin{array}{l}\mathbf{N}_{2, S_{2}} \\ \mathbf{N}_{2, \bar{S}_{2}}\end{array}\right)$.

- $\mathbf{R}_{0} \leftarrow \mathbb{Z}_{p}^{\bar{K}_{0} \times k} ; \mathbf{R}_{1} \leftarrow \mathbb{Z}_{p}^{\bar{K}_{1} \times k} ; \mathbf{R}_{2} \leftarrow \mathbb{Z}_{p}^{\bar{K}_{2} \times k}$.

- It computes $[\mathbf{B}]_{1}:=\left[\mathbf{B}^{\dagger}\right]_{1}+[\mathbf{M}]_{1}^{\top} \mathbf{G}^{\top} \mathbf{R}_{0}+\left[\mathbf{N}_{1}\right]_{1}^{\top} \mathbf{H}^{\top} \mathbf{R}_{1}$ and $[\mathbf{D}]_{2}:=\left[\mathbf{D}^{\dagger}\right]_{2}+\left[\mathbf{N}_{2}\right]_{2}^{\top} \mathbf{F}^{\top} \mathbf{R}_{2}$

- It computes $\left[\mathbf{C}_{1}\right]_{2}:=\left(\begin{array}{cc}\mathbf{T}_{\mathbf{G}} & \mathbf{0} \\ \mathbf{0} & \mathbf{T}_{\mathbf{H}}\end{array}\right)\left[\mathbf{C}_{1}^{\dagger}\right]_{2}+\left(\begin{array}{l}\mathbf{R}_{0} \\ \mathbf{R}_{1}\end{array}\right)[\mathbf{A}]_{2}$ and $\left[\mathbf{C}_{2}\right]_{1}:=\mathbf{T}_{\mathbf{F}}\left[\mathbf{C}_{2}^{\dagger}\right]_{1}+\mathbf{R}_{2}[\mathbf{A}]_{1}$.

- It sets

$$
\begin{gathered}
\rho:=\left([\mathbf{G}]_{1},[\mathbf{H}]_{1},[\mathbf{F}]_{2},[\mathbf{M}]_{1},\left[\mathbf{N}_{1}\right]_{1},\left[\mathbf{N}_{2}\right]_{2}\right), \quad h_{\mathbf{l s}}(\theta):=\left(\mathbf{G}, \mathbf{H}, \mathbf{F}, \mathbf{N}_{1}, \mathbf{N}_{2}\right) \\
\operatorname{crs}:=\left([\mathbf{B}]_{1},[\mathbf{D}]_{2},[\mathbf{A}]_{1,2},\left[\mathbf{C}_{1}\right]_{2},\left[\mathbf{C}_{2}\right]_{1}\right)
\end{gathered}
$$

It then executes $\mathcal{A}\left(\rho, h_{\mathrm{|s}}(\theta)\right.$, crs $)$ until it outputs a statement $\left([\boldsymbol{c}]_{1},\left[\boldsymbol{d}_{1}\right]_{1},\left[\boldsymbol{d}_{2}\right]_{2}, \boldsymbol{w}\right)$ together with an accepting proof $[\pi]_{1},[\boldsymbol{\theta}]_{2}$. Given an accepting proof $\mathcal{B}_{S}$ sets $\left[\boldsymbol{x}^{\dagger}\right]_{1}=\mathbf{T}_{\mathrm{G}}[c]_{1},\left[\boldsymbol{y}_{1}^{\dagger}\right]_{1}=$ $\mathbf{T}_{\mathbf{H}}\left[\boldsymbol{d}_{1}\right]_{1},\left[\boldsymbol{y}_{2}^{\dagger}\right]_{2}=\mathbf{T}_{\mathbf{F}}\left[\boldsymbol{d}_{2}\right]_{2},\left[\boldsymbol{\pi}^{\dagger}\right]_{1}=[\boldsymbol{\pi}]_{1}-[\boldsymbol{c}]_{1}^{\top} \mathbf{R}_{0}-\left[\boldsymbol{d}_{1}\right]_{1}^{\top} \mathbf{R}_{1}$ and $\left[\boldsymbol{\theta}^{\dagger}\right]_{2}=[\boldsymbol{\theta}]_{1}-\left[\boldsymbol{d}_{2}\right]_{2}^{\top} \mathbf{R}_{2}$. It outputs $\left(\left(\left[\boldsymbol{x}^{\dagger}\right]_{1},\left[\boldsymbol{y}_{1}^{\dagger}\right]_{1},\left[\boldsymbol{y}_{2}^{\dagger}\right]_{2}\right), \boldsymbol{w},\left(\left[\boldsymbol{\pi}^{\dagger}\right]_{1},\left[\boldsymbol{\theta}^{\dagger}\right]_{2}\right)\right)$.

Note that the commitment keys are perfectly binding at $S$. First, we claim that in this case the values $\rho, h_{\mathrm{ls}}(\theta)$, crs output by $\mathcal{B}_{S}$ are identically distributed to honestly computed ones and thus do not skew the probability that $\mathcal{A}$ outputs a valid proof. For $\rho, h_{\mathrm{ss}}(\theta)$, this is immediate by the witness samplability of the distributions $\mathcal{M}, \mathcal{N}_{1}, \mathcal{N}_{2}$. We show that this holds for crs as well. 
Let $\mathbf{K}_{0}^{\dagger} \in \mathbb{Z}_{p}^{\left|S_{1}\right| \times k}, \mathbf{K}_{1}^{\dagger} \in \mathbb{Z}_{p}^{\left|S_{2}\right| \times k}, \mathbf{K}_{2}^{\dagger} \in \mathbb{Z}_{p}^{\left|S_{2}\right| \times k}$ be the implicit values used to compute crs ${ }^{\dagger}$, that is, they satisfy

$$
\mathbf{B}^{\dagger}=\mathbf{M}_{S}^{\top} \mathbf{K}_{0}^{\dagger}+\mathbf{N}_{1, S}^{\top} \mathbf{K}_{1}^{\dagger}, \mathbf{D}^{\dagger}=\mathbf{N}_{2, S}^{\top} \mathbf{K}_{2}^{\dagger}, \mathbf{C}_{1}^{\dagger}=\left(\begin{array}{l}
\mathbf{K}_{0}^{\dagger} \\
\mathbf{K}_{1}^{\dagger}
\end{array}\right) \mathbf{A} \text { and } \mathbf{C}_{2}^{\dagger}=\mathbf{K}_{2}^{\dagger} \mathbf{A} .
$$

Now $\mathcal{B}_{S}$ implicitly defines $\mathbf{K}_{2}=\mathbf{T}_{\mathbf{G}} \mathbf{K}_{0}^{\dagger}+\mathbf{R}_{0}, \mathbf{K}_{2}=\mathbf{T}_{\mathbf{H}} \mathbf{K}_{1}^{\dagger}+\mathbf{R}_{1}, \mathbf{K}_{2}=\mathbf{T}_{\mathbf{F}} \mathbf{K}_{2}^{\dagger}+\mathbf{R}_{2}$. First, note that these matrices are uniformly distributed since $\mathbf{R}_{0}, \mathbf{R}_{1}, \mathbf{R}_{2}$ are uniformly distributed. Thus $\mathbf{K}_{1}, \mathbf{K}_{2}, \mathbf{K}_{2}$ are distributed identically to honestly generated values for generating a crs. We claim that the crs output by $\mathcal{A}$ is identically distributed to sampling this matrix and computing the other values honestly. Indeed we have that

$$
\begin{aligned}
\mathbf{B} & =\mathbf{B}^{\dagger}+\mathbf{M}^{\top} \mathbf{G}^{\top} \mathbf{R}_{0}+\mathbf{N}_{1}^{\top} \mathbf{H}^{\top} \mathbf{R}_{1} \\
& =\mathbf{M}_{S_{1}}^{\top} \mathbf{K}_{1}^{\dagger}+\mathbf{N}_{1, S_{2}}^{\top} \mathbf{K}_{2}^{\dagger}+\mathbf{M}^{\top} \mathbf{G}^{\top} \mathbf{R}_{0}+\mathbf{N}_{1}^{\top} \mathbf{H}^{\top} \mathbf{R}_{1} \\
& =\mathbf{M}^{\top} \mathbf{G}^{\top} \mathbf{T}_{\mathbf{G}} \mathbf{K}_{1}^{\dagger}+\mathbf{N}_{1}^{\top} \mathbf{H}^{\top} \mathbf{T}_{\mathbf{H}}^{\top} \mathbf{K}_{2}^{\dagger}+\mathbf{M}^{\top} \mathbf{G}^{\top} \mathbf{R}_{0}+\mathbf{N}_{1}^{\top} \mathbf{H}^{\top} \mathbf{R}_{1} \\
& =\mathbf{M}^{\top} \mathbf{G}^{\top}\left(\mathbf{T}_{\mathbf{G}} \mathbf{K}_{1}^{\dagger}+\mathbf{R}_{0}\right)+\mathbf{N}_{1}^{\top} \mathbf{H}^{\top}\left(\mathbf{T}_{\mathbf{H}}^{\top} \mathbf{K}_{2}^{\dagger}+\mathbf{R}_{1}\right) \\
& =\mathbf{M}^{\top} \mathbf{G}^{\top} \mathbf{K}_{1}+\mathbf{N}_{1}^{\top} \mathbf{H}^{\top} \mathbf{K}_{2}
\end{aligned}
$$

where the third equality follows since by the local extractability of the SSBs we have that $\mathbf{T}_{\mathbf{G}}^{\top} \mathbf{G M}=\mathbf{M}_{S_{1}}, \mathbf{T}_{\mathbf{H}}^{\top} \mathbf{H N}_{1}=\mathbf{N}_{1, S_{2}}$. Similarly, we have

$$
\begin{aligned}
\mathbf{D} & =\mathbf{D}^{\dagger}+\mathbf{N}_{2}^{\top} \mathbf{F}^{\top} \mathbf{R}_{2} \\
& =\mathbf{N}_{2, S_{2}}^{\top} \mathbf{K}_{3}^{\dagger}+\mathbf{N}_{2}^{\top} \mathbf{F}^{\top} \mathbf{R}_{2} \\
& =\mathbf{N}_{2}^{\top} \mathbf{F}^{\top} \mathbf{T}_{\mathbf{F}} \mathbf{K}_{2}^{\dagger}+\mathbf{N}_{2}^{\top} \mathbf{F}^{\top} \mathbf{R}_{2} \\
& =\mathbf{N}_{2}^{\top} \mathbf{F}^{\top}\left(\mathbf{T}_{\mathbf{F}} \mathbf{K}_{2}^{\dagger}+\mathbf{R}_{2}\right) \\
& =\mathbf{N}_{2}^{\top} \mathbf{F}^{\top} \mathbf{K}_{2}
\end{aligned}
$$

Also, we have that

$$
\begin{aligned}
& \mathbf{C}_{1}=\left(\begin{array}{cc}
\mathbf{T}_{\mathbf{G}} & \mathbf{0} \\
\mathbf{0} & \mathbf{T}_{\mathbf{H}}
\end{array}\right) \mathbf{C}_{1}^{\dagger}+\left(\begin{array}{l}
\mathbf{R}_{0} \\
\mathbf{R}_{1}
\end{array}\right) \mathbf{A}=\left(\begin{array}{cc}
\mathbf{T}_{\mathbf{G}} & \mathbf{0} \\
\mathbf{0} & \mathbf{T}_{\mathbf{H}}
\end{array}\right)\left(\begin{array}{l}
\mathbf{K}_{1}^{\dagger} \\
\mathbf{K}_{2}^{\dagger}
\end{array}\right) \mathbf{A}+\left(\begin{array}{l}
\mathbf{R}_{0} \\
\mathbf{R}_{1}
\end{array}\right) \mathbf{A}=\left(\begin{array}{c}
\mathbf{T}_{\mathbf{G}} \mathbf{K}_{1}^{\dagger}+\mathbf{R}_{0} \\
\mathbf{T}_{\mathbf{H}} \mathbf{K}_{2}^{\dagger}+\mathbf{R}_{1}
\end{array}\right) \mathbf{A}=\left(\begin{array}{l}
\mathbf{K}_{1} \\
\mathbf{K}_{2}
\end{array}\right) \mathbf{A} \\
& \mathbf{C}_{2}=\mathbf{T}_{\mathbf{F}} \mathbf{C}_{2}^{\dagger}+\mathbf{R}_{2} \mathbf{A}=\mathbf{T}_{\mathbf{F}} \mathbf{K}_{2}^{\dagger} \mathbf{A}+\mathbf{R}_{2} \mathbf{A}=\left(\mathbf{T}_{\mathbf{F}} \mathbf{K}_{2}^{\dagger}+\mathbf{R}_{2}\right) \mathbf{A}=\mathbf{K}_{2} \mathbf{A}
\end{aligned}
$$

so the outputted crs is indeed identically distributed to an honest one.

Then, we show that $\mathcal{B}$ outputs a valid statement-proof pair w.r.t. to $\mathrm{crs}^{\dagger}$. Since the commitment keys are extractable and perfectly binding at $S$, we have that $\boldsymbol{x}^{\dagger}, \boldsymbol{y}_{1}^{\dagger}$ and $\boldsymbol{y}_{2}^{\dagger}$ are valid openings for the commitments given. Assuming $\mathcal{A}$ produces a valid statement for $\mathcal{R}_{\rho, S^{\prime}}^{\text {no }}$, for the extracted values it holds that $\boldsymbol{x}^{\dagger}=\mathbf{M}_{S_{1}} \boldsymbol{w}$ and either $\boldsymbol{y}_{1}^{\dagger} \neq \mathbf{N}_{1, S_{2}} \boldsymbol{w}$ or $\boldsymbol{y}_{2}^{\dagger} \neq \mathbf{N}_{2, S_{2}} \boldsymbol{w}$. Thus, $\mathcal{B}_{S}$ outputs a valid statement and it suffices to show that $\left[\pi^{\dagger}\right]_{1},\left[\boldsymbol{\theta}^{\dagger}\right]_{2}$ is a valid proof. Indeed, we 
have that

$$
\begin{aligned}
& \mathbf{0}=\boldsymbol{\pi} \mathbf{A}+\boldsymbol{\theta} \mathbf{A}-\left(\boldsymbol{c}^{\top} \mid \boldsymbol{d}_{1}^{\top}\right) \mathbf{C}_{1}-\boldsymbol{d}_{2}^{\top} \mathbf{C}_{2} \\
& =\left(\pi^{\dagger}+c^{\top} \mathbf{R}_{0}+d_{1}^{\top} \mathbf{R}_{1}\right) \mathbf{A}+\left(\boldsymbol{\theta}^{\dagger}+d_{2}^{\top} \mathbf{R}_{2}\right) \mathbf{A} \\
& -\left(c^{\top} \mid d_{1}^{\top}\right)\left(\left(\begin{array}{cc}
\mathbf{T}_{\mathbf{G}} & \mathbf{0} \\
\mathbf{0} & \mathbf{T}_{\mathbf{H}}
\end{array}\right) \mathbf{C}_{1}^{\dagger}+\left(\begin{array}{l}
\mathbf{R}_{0} \\
\mathbf{R}_{1}
\end{array}\right) \mathbf{A}\right) \\
& -d_{2}^{\top}\left(\mathbf{T}_{\mathrm{F}} \mathbf{C}_{2}^{\dagger}+\mathbf{R}_{2} \mathrm{~A}\right) \\
& =\left(\pi^{\dagger}+c^{\top} \mathbf{R}_{0}+d_{1}^{\top} \mathbf{R}_{2}\right) \mathbf{A}+\left(\boldsymbol{\theta}^{\dagger}+d_{2}^{\top} \mathbf{R}_{2}\right) \mathbf{A} \\
& -\left(c^{\top} \mathbf{T}_{\mathbf{G}} \mid d_{1}^{\top} \mathbf{T}_{\mathbf{H}}\right) \mathbf{C}_{1}^{\dagger}-\left(c^{\top} \mathbf{R}_{0}-d_{1}^{\top} \mathbf{R}_{1}\right) \mathbf{A} \\
& -d_{2}^{\top} \mathrm{T}_{\mathrm{F}} \mathrm{C}_{2}^{\dagger}-d_{2}^{\top} \mathbf{R}_{2} \mathrm{~A} \\
& =\pi^{\dagger} \mathbf{A}+\boldsymbol{\theta}^{\dagger} \mathbf{A}-\left(c^{\top} \mathbf{T}_{\mathbf{G}} \mid d_{1}^{\top} \mathbf{T}_{\mathbf{H}}\right) \mathbf{C}_{1}^{\dagger}-d_{2}^{\top} \mathbf{T}_{\mathbf{F}} \mathbf{C}_{2}^{\dagger} \\
& =\pi^{\dagger} \mathrm{A}+\boldsymbol{\theta}^{\dagger} \mathrm{A}-\left(\boldsymbol{x}^{\dagger^{\top}} \mid \boldsymbol{y}_{1}^{\dagger^{\top}}\right) \mathrm{C}_{1}^{\dagger}-\boldsymbol{y}_{2}^{\dagger^{\top}} \mathrm{C}_{2}^{\dagger}
\end{aligned}
$$

and the last equation is the verification equation for the knowledge transfer argument for $\mathrm{crs}^{\dagger}$.

We next show that when the distribution $\mathcal{M}, \mathcal{N}_{1}, \mathcal{N}_{2}$ guarantee that the linear knowledge transfer argument is secure w.r.t. all possible sets $S$, construction QABlin has $h_{\mathrm{Is}}$-strong local knowledge soundness where $h_{\mathrm{ls}}$ includes $\mathbf{G}, \mathbf{H}, \mathbf{F}, \mathbf{N}_{1}, \mathbf{N}_{2}$, and some extra information about the matrix $\mathbf{M}$.

Corollary 4. Let $\mathcal{D}_{k}$ be a matrix distribution for which $\mathcal{D}_{k}$-SKerMDH. Denote $\mathcal{M}_{S}$ (resp. $\mathcal{N}_{1, S}, \mathcal{N}_{2, S}$ ) the distributions that sample matrices from $\mathcal{M}$ (res. $\mathcal{N}_{1}, \mathcal{N}_{1}$ ), and restricts them to rows corresponding to $S$. Then

1. If for all $S_{1} \subseteq[d]$ with $S_{1} \leq K_{1},\left(\mathcal{M}_{S_{1}}^{\top}, h\right)-\mathrm{MDDH}$ holds, QABlin is an $h_{\mathrm{Is}}$-strong local knowledge sound proof system, where $h_{\mathrm{Is}}(\theta)=\left(h\left(\mathbf{M}_{S}\right), \mathbf{G}, \mathbf{H}, \mathbf{F}, \mathbf{N}_{1}, \mathbf{N}_{2}\right)$.

2. If for all $S_{1}, S_{2} \subseteq[d]$ with $S_{1} \leq K_{1}, S_{2} \leq K_{2}$ the distributions $\mathcal{M}_{S_{1}}, \mathcal{N}_{S_{2}}, \mathcal{N}_{S_{2}}$ output matrices with the last $n^{\prime}$ columns being $\mathbf{0}$, and $\left(\mathcal{M}_{S_{1}}^{\prime}, h\right)-\mathrm{MDDH}$ holds, with $\mathcal{M}_{S_{1}}^{\prime}$ being $\mathcal{M}_{S_{1}}$ where we delete the trailing zero columns, then QABlin is an his-strong local knowledge sound proof system, where $h_{\mathbf{l s}}(\theta)=\left(h\left(\mathbf{M}_{S}\right), \mathbf{G}, \mathbf{H}, \mathbf{F}, \mathbf{N}_{1}, \mathbf{N}_{2}\right)$.

Proof. The proof is an immediate consequence of of Thm. 10 and Thm. 19.1 for case 1 and Thm. 19.2 for case 2 .

The proof of oblivious trapdoor generation follows from the oblivious trapdoor generation and index set hiding of SSB commitments. We follow essentially the same proof as in the unilateral case.

First we show that we construct an indistinguishable crs given only the commitment keys and the matrices $\mathbf{M}, \mathbf{N}_{1}, \mathbf{N}_{2}$.

Lemma 2. There exists a modified crs generation algorithm $\mathrm{K}^{\prime}$ that on input $\left(\rho, \theta^{\prime}\right)$, where $\theta^{\prime}$ contains only either $\mathbf{M}, \mathbf{N}_{1}, \mathbf{N}_{2}$ or $\mathbf{G}, \mathbf{H}, \mathbf{F}$ outputs a crs such that ( $\rho$, crs) are identically distributed to the honest algorithm. 
The lemma follows directly by noting that $[\mathbf{B}]_{1},[\mathbf{D}]_{2}$ are efficiently computable given the commitment keys and the discrete logarithms of matrices $\mathbf{M}, \mathbf{N}_{1}, \mathbf{N}_{2}$ (equivalently $\mathbf{G}, \mathbf{H}, \mathbf{F}$ ). As in the unilateral case, we abuse notation and refer to $\mathrm{K}^{\prime}\left(\rho, \theta^{\prime}\right)$ as $\mathrm{K}\left(\rho, \theta^{\prime}\right)$.

In the next theorem we consider the three keys issued as a single key. It is easy to verify that the properties of the commitment keys still hold. Essentially, we want to capture the condition that the keys preserve oblivious key generation even if we consider a function $h$ that outputs information that depends on all commitment keys. In our delegation construction this will correspond to $h(\mathbf{G}, \mathbf{H}, \mathbf{F})=\left([\mathbf{G}]_{1},[\mathbf{H}]_{2},[\mathbf{F}]_{2},[\mathbf{H} \otimes \mathbf{F}-\mathbf{Z}]_{1},[\mathbf{Z}]_{2}\right)$, for a uniform $\mathbf{Z}$, namely the information needed to obliviously create a crs for the kronecker composition of the last two keys.

Theorem 11. Let $\mathcal{M}, \mathcal{N}_{1}, \mathcal{N}_{2}$ be witness samplable distributions, and CS be an algebraic SSB commitment scheme and let $\mathrm{CS}^{\prime}$ be the concatenation of three instances of $\mathrm{CS}$, that is it outputs $\mathbf{G}^{\prime}=$ $\left(\begin{array}{ccc}{\left[\mathbf{G}_{0}\right]_{1}} & \mathbf{0} & \mathbf{0} \\ \mathbf{0} & {\left[\mathbf{G}_{1}\right]_{1}} & \mathbf{0} \\ \mathbf{0} & \mathbf{0} & {\left[\mathbf{G}_{2}\right]_{2}}\end{array}\right)$ with $\mathbf{G}_{i} \leftarrow \mathrm{CS}$.KeyGen $\left(g k, n, d, K_{i}, S_{i}\right)$. If $\mathrm{CS}^{\prime}$ has h-strong oblivious trapdoor generation, then construction QABlin of Fig. 5 is $h_{n s}$-strong oblivious where $h_{n s}=\left(h(s k), \mathbf{M}_{1}, \mathbf{N}_{1}, \mathbf{N}_{2}\right)$. Furthermore,

1. For every PPT $\mathcal{A}$ against $h_{n s}$-strong index set hiding of $Q A B$ lin, there exists an adversary $\mathcal{B}$ against h-index set hiding property of CS, such that $\operatorname{Adv}_{\mathrm{ISH}}^{Q A B \text { lin }}(\mathcal{A}) \leq 3 \operatorname{Adv}_{\mathrm{ISH}}^{\mathrm{CS}}(\mathcal{B})$.

2. For every $\mathcal{A}$ against oblivious trapdoor generation of $Q A B$ lin, there exists an adversary $\mathcal{B}$ against oblivious trapdoor of CS, such that $\operatorname{Adv}_{\text {oblv }}^{Q A B l i n}(\mathcal{A}) \leq 3 \operatorname{Adv}_{\text {oblv }}^{C S}(\mathcal{B})$.

Proof. Since the commitment key is perfectly binding at the extraction set, it is enough to show that $h_{n s}$-strong index set hiding holds and that we can sample a tuple $(\rho, \mathrm{crs})$ indistinguishable from the one we are given, along with a valid trapdoor.

For index set hiding, it is enough to notice that the crs of QABlin can be efficiently computed given only $[\mathbf{G}]_{1},[\mathbf{H}]_{1},[\mathbf{F}]_{2}$. Indeed by sampling $[\mathbf{M}]_{1}, \mathbf{M} \leftarrow \mathcal{M},\left[\mathbf{N}_{1}\right]_{1}, \mathbf{N}_{1} \leftarrow \mathcal{N}_{1},\left[\mathbf{N}_{2}\right]_{2}, \mathbf{N}_{2} \leftarrow$ $\mathcal{N}_{2}$ all values of $\mathrm{crs}$ are efficiently computable as noted in Lemma 2. Thus, a distinguishing advantage in index set hiding of QABlin immediately implies equal advantage on the respective property of CS.

For oblivious crs generation we first describe the OblSetup algorithm. Let $S^{\prime} \subseteq S$.

$$
\begin{aligned}
\text { ObISetup } & \left(\rho:=\left([\mathbf{G}]_{1},[\mathbf{H}]_{1},[\mathbf{F}]_{2},[\mathbf{M}]_{1},\left[\mathbf{N}_{1}\right]_{1},\left[\mathbf{N}_{2}\right]_{2}\right), \text { crs }\right): \\
& \bullet\left(\left[\mathbf{G}^{\prime}\right]_{1}, \mathbf{T}_{\mathbf{G}}^{\prime}\right) \leftarrow \text { CS.ObISetup }\left(g k, d, K_{0}, S_{0},[\mathbf{G}]_{1}\right) . \\
& \bullet\left(\left[\mathbf{H}^{\prime}\right]_{1}, \mathbf{T}_{\mathbf{H}}^{\prime}\right) \leftarrow \text { CS.ObISetup }\left(g k, d, K_{1}, S_{1},[\mathbf{H}]_{1}\right) . \\
& -\left(\left[\mathbf{F}^{\prime}\right]_{2}, \mathbf{T}_{\mathbf{F}}^{\prime}\right) \leftarrow \text { CS.ObISetup }\left(g k, d, K_{2}, S_{2},[\mathbf{F}]_{2}\right) . \\
& \bullet \text { Sample }\left(\left[\mathbf{M}^{\prime}\right]_{1}, \mathbf{M}^{\prime}\right) \leftarrow \mathcal{M} ;\left(\left[\mathbf{N}_{1}^{\prime}\right]_{1}, \mathbf{N}_{1}^{\prime}\right) \leftarrow \mathcal{N}_{2} ;\left(\left[\mathbf{N}_{2}^{\prime}\right]_{2}, \mathbf{N}_{2}^{\prime}\right) \leftarrow \mathcal{N}_{2} ; \\
& \bullet \text { Set } \tau^{\prime}=\left(\mathbf{T}_{\mathbf{G}^{\prime}}^{\prime} \mathbf{T}_{\mathbf{H}^{\prime}}^{\prime}, \mathbf{T}_{\mathbf{F}}^{\prime}\right) \text { and compute crs } \leftarrow \operatorname{QABlin} . \mathrm{K}\left(\rho, \theta^{\prime}=\left(\mathbf{M}, \mathbf{N}_{1}, \mathbf{N}_{2}\right)\right) .
\end{aligned}
$$

Note that the only difference in sampling with $S$ and with $S^{\prime}$ is how we sample the commitment keys $\mathbf{G}, \mathbf{H}, \mathbf{F}$; crs is identically distributed to an honest one since we sample $\mathbf{M}, \mathbf{N}_{1}, \mathbf{N}_{2}$ in the same way that $\mathcal{D}_{\text {par }}$ does. Also, by oblivious key generation of $C S$, the trapdoor $\tau^{\prime}$ is a valid one w.r.t. $\mathbf{G}^{\prime}, \mathbf{H}^{\prime}, \mathbf{F}^{\prime}$ and set $S^{\prime}$, so it extracts valid witnesses which, by perfect binding in $S^{\prime}$ are unique and do not assist the distinguisher which can compute them itself.

Finally, we get the following corollary. 
Corollary 5. When CS is the one from fig. 3 and $\mathrm{CS}^{\prime}$ is the concatenation of the three keys as described in Thm. 11 for and $h(\mathbf{G}, \mathbf{H}, \mathbf{F})=\left([\mathbf{H} \otimes \mathbf{F}-\mathbf{Z}]_{1},[\mathbf{Z}]_{2}\right)$ for uniform $\mathbf{Z}$, then QABlin from fig. 5 is $h_{n s}$-strong no-signaling where $h_{n s}(\theta)=\left(h(\mathbf{G}, \mathbf{H}, \mathbf{F}), \mathbf{M}, \mathbf{N}_{1}, \mathbf{N}_{2}\right)$.

Proof. Follows directly from Thm. 7, the $h_{n s}$-strong ISH of the QALin which we show on Thm. 11 and the properties of the kronecker key operator (Thm. 6). 


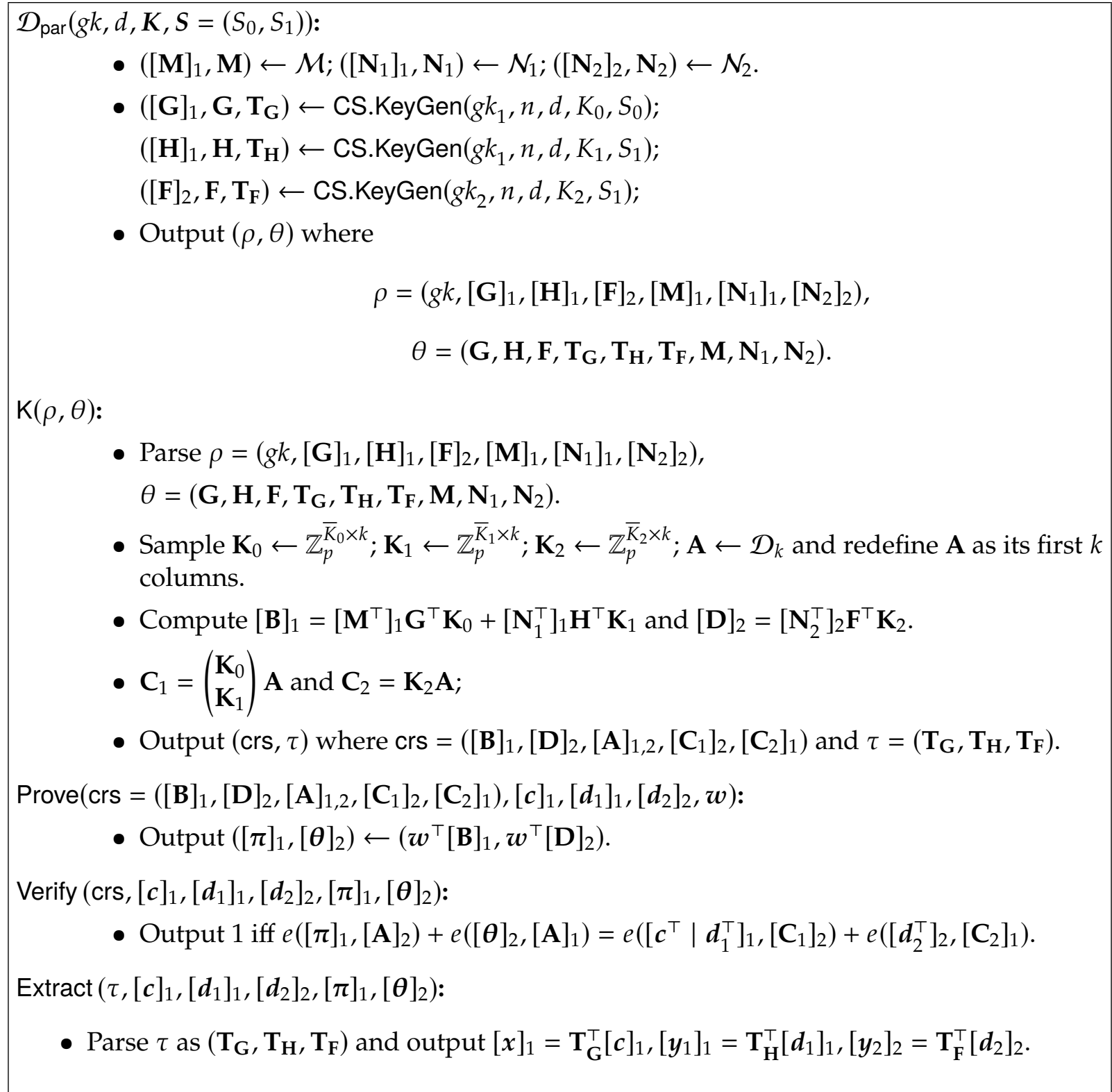

Figure 5: Quasi argument QABlin for knowledge transfer of membership in linear space. 
Quasi Argument for Sum Knowledge Transfer. Let $\left(\mathcal{M}_{1}, \mathcal{M}_{1}\right)$ be some (possibly correlated) witness samplable distributions outputting matrices in $\mathbb{G}_{1}^{d \times n} \times \mathbb{G}_{2}^{d \times n}$ and $\mathcal{N}$ be witness samplable distributions outputting matrices in $\mathbb{G}_{1}^{d \times n}$ for $n, d \in \mathbb{N}$. Let $\boldsymbol{K} \leq d$ where $\boldsymbol{K}=\left(K_{0}, K_{1}\right)$ and $S \subseteq([d],[d])$ where $S=S_{1} \cup S_{2}$ and $S \leq K$. Let $C S$ be an algebraic SSB commitment scheme and $\mathrm{CS}^{\prime}$ be a split algebraic commitment key with commitment space $\mathbb{G}_{1}^{\bar{K}}, \mathbb{G}_{1}^{\bar{K}} \times \mathbb{G}_{2}^{\bar{K}}$ respectively. The parameter language is

$$
\begin{aligned}
& \mathcal{L}_{\text {par }}=\left\{\left[\mathbf{M}_{1}\right]_{1},\left[\mathbf{M}_{2}\right]_{2},[\mathbf{N}]_{1},\left[\mathbf{Q}_{1}\right]_{1},\left[\mathbf{Q}_{2}\right]_{1},[\mathbf{F}]_{2} \mid \exists \mathbf{M}_{1}, \mathbf{M}_{2}, \mathbf{N}_{1}, \mathbf{Q}_{1}, \mathbf{Q}_{2}, \mathbf{F}\right. \text { s.t. } \\
& \left(\left[\mathbf{M}_{1}\right]_{1},\left[\mathbf{M}_{2}\right]_{2}, \mathbf{M}_{1}, \mathbf{M}_{2}\right) \in \operatorname{Sup}\left(\mathcal{M}_{1}, \mathcal{M}_{2}\right),\left([\mathbf{N}]_{1}, \mathbf{N}\right) \in \operatorname{Sup}(\mathcal{N}), \\
& \left(\left[\mathbf{Q}_{1}\right]_{1},\left[\mathbf{Q}_{2}\right]_{2}, \mathbf{Q}_{1}, \mathbf{Q}_{2}, \mathbf{T}_{\mathbf{Q}}\right) \in \operatorname{Sup}\left(\mathrm{CS}^{\prime} . \operatorname{KeyGen}\left(g k, n, K_{0}, S_{1}\right)\right) \text {, } \\
& \left.\left([\mathbf{F}]_{1}, \mathbf{F}, \mathbf{T}_{\mathbf{F}}\right) \in \operatorname{Sup}\left(\text { CS.KeyGen }\left(g k_{1}, n, K_{1}, S_{2}\right)\right)\right\}
\end{aligned}
$$

We assume w.l.o.g. that the corresponding relation is efficiently verifiable. The parameters $\rho=\left([\mathbf{M}]_{1},\left[\mathbf{N}_{1}\right]_{1},\left[\mathbf{N}_{2}\right]_{2},\left[\mathbf{Q}_{1}\right]_{1},\left[\mathbf{Q}_{2}\right]_{1},[\mathbf{F}]_{2}\right)$ define the following relations ${ }^{18}$

$$
\begin{aligned}
& \mathcal{R}_{\rho}^{\mathrm{yes}}=\left\{\left[c_{1}\right]_{1},\left[c_{2}\right]_{2},[d]_{2}, \boldsymbol{w} \mid\left(\begin{array}{c}
c_{1}+c_{2} \\
\boldsymbol{d}
\end{array}\right)=\left(\begin{array}{c}
\left(\mathbf{Q}_{1}+\mathbf{Q}_{2}\right)\left(\mathbf{M}_{1}+\mathbf{M}_{2}\right) \\
\mathbf{F N}
\end{array}\right) \boldsymbol{w}\right\},
\end{aligned}
$$

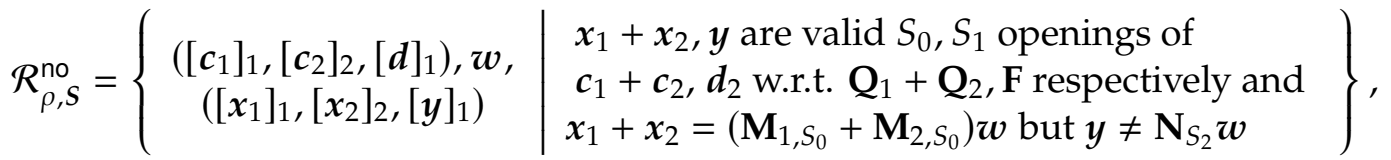

that is the partial witness for $S$ is some valid local openings $\left[x_{1}\right]_{1},\left[x_{2}\right]_{2},[y]_{1}$ w.r.t. to $\mathrm{G}, \mathbf{H}, \mathbf{F}$ respectively that satisfy the following: if $x_{1}+x_{2}=\left(\mathbf{M}_{1, S_{1}}+\mathbf{M}_{2, S_{1}}\right) \boldsymbol{w}$ then it should be the case that $\boldsymbol{y}=\mathbf{N}_{S_{2}} \boldsymbol{w}$ where $\boldsymbol{w}$ is the promise of the adversary. Note that if $S_{1}$ is the empty set the latter relations trivially hold.

We present the protocol in Fig 6.

Theorem 12. Let $\mathcal{M}_{1}, \mathcal{M}_{2}$ be (possibly correlated) witness samplable distribution, $\mathcal{N}$ be a witness samplable distribution, $\mathcal{D}_{k}$ a matrix distribution and $\mathrm{CS}, \mathrm{CS}^{\prime}$ an algebraic and split algebraic SSB commitment respectively with perfect completeness. Also, let $\mathcal{A}$ be an adversary against $h_{l s}$-strong local soundness of construction QASum where $h_{l s}=\left(\mathbf{Q}_{1}, \mathbf{Q}_{2}, \mathbf{F}, \mathbf{N}\right)$. Then, QAsum has perfect completeness and $h_{l s}$-strong local knowledge soundness holds with probability at least $1-\operatorname{Adv}_{\text {snd }}^{\Pi_{k t-s u m}}\left(\mathcal{B}_{S}\right)$, where $\mathcal{B}_{S}$ is any PPT adversary against soundness of $\Pi_{k t-s u m}$.

Proof. For completeness, we have that

$$
\begin{aligned}
\left(\boldsymbol{c}_{1}^{\top} \mid \boldsymbol{d}^{\top}\right) \mathbf{C}_{1}+\boldsymbol{c}_{2}^{\top} \mathbf{C}_{2} & =\left(c_{1}^{\top} \mid \boldsymbol{d}^{\top}\right)\left(\begin{array}{l}
\mathbf{K}_{0} \\
\mathbf{K}_{1}
\end{array}\right) \mathbf{A}+\boldsymbol{c}_{2}^{\top} \mathbf{K}_{0} \mathbf{A} \\
& =\left(c_{1}^{\top} \mathbf{K}_{0}+\boldsymbol{d}^{\top} \mathbf{K}_{1}+\boldsymbol{c}_{2}^{\top} \mathbf{K}_{0}\right) \mathbf{A} \\
& =\left(\left(c_{1}^{\top}+c_{2}^{\top}\right) \mathbf{K}_{0}+\boldsymbol{d}^{\top} \mathbf{K}_{1}\right) \mathbf{A} \\
& =\left(\boldsymbol{w}^{\top}\left(\mathbf{M}_{1}^{\top}+\mathbf{M}_{2}^{\top}\right)\left(\mathbf{Q}_{1}^{\top}+\mathbf{Q}_{2}^{\top}\right) \mathbf{K}_{0}+\boldsymbol{w}^{\top} \mathbf{N}^{\top} \mathbf{F}^{\top} \mathbf{K}_{1}\right) \mathbf{A} \\
& =\boldsymbol{w}^{\top}\left(\left(\mathbf{M}_{1}^{\top}+\mathbf{M}_{2}^{\top}\right) \mathbf{Q}^{\top} \mathbf{K}_{0}+\mathbf{N}^{\top} \mathbf{F}^{\top} \mathbf{K}_{1}\right) \mathbf{A} \\
& =\boldsymbol{w}^{\top}\left(\left(\mathbf{M}_{1}^{\top} \mathbf{Q}^{\top} \mathbf{K}_{0}+\mathbf{N}^{\top} \mathbf{F}^{\top} \mathbf{K}_{1}+\mathbf{Z}\right)+\left(\mathbf{M}_{2}^{\top} \mathbf{Q}^{\top} \mathbf{K}_{0}-\mathbf{Z}\right)\right) \mathbf{A} \\
& =\boldsymbol{w}^{\top}(\mathbf{B}+\mathbf{D}) \mathbf{A} \\
& =\boldsymbol{w}^{\top} \mathbf{B} \mathbf{A}+\boldsymbol{w}^{\top} \mathbf{D A} \\
& =\pi \mathbf{A}+\boldsymbol{\theta} \mathbf{A}
\end{aligned}
$$

${ }^{18}$ We allow both the distributions $\mathcal{M}_{1}, \mathcal{M}_{2}, \mathcal{N}$ and the commitment keys to include some auxiliary information with its associated witness which are included in $\rho, \theta$ respectively. This auxiliary information is not used in the protocol, but is public when the protocol is used inside other protocol. We omit it here to simplify the presentation but we consider it whenever needed. 


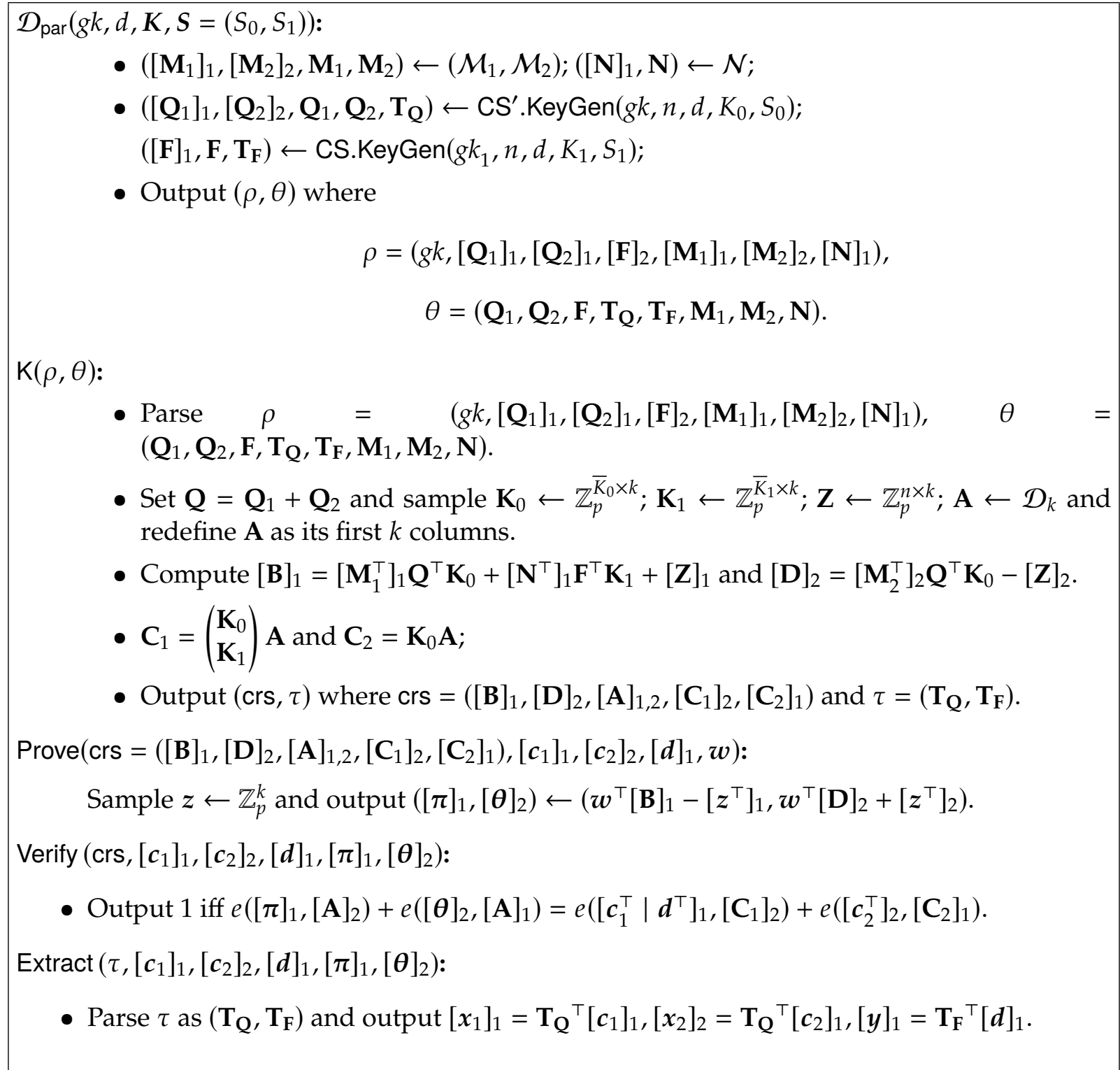

Figure 6: Quasi argument QASum for knowledge transfer of sum membership in linear space.

Local knowledge soundness follows using almost an identical argument to Thm. 10 and reducing to knowledge transfer of KTA Sum Argument $\Pi_{\text {kt-sum }}$ of Fig. 1 . Given an adversary $\mathcal{A}$ breaking Knowledge Transfer of the quasi-argument of Fig. 6, we construct another adversary $\mathcal{B}_{S}$ that breaks Knowledge Transfer of the argument $\Pi_{\mathrm{kt} \text {-sum }}$ for matrices $\left[\mathbf{M}_{1, S_{0}}\right]_{1},\left[\mathbf{M}_{2, S_{0}}\right]_{2}$ and $\left[\mathbf{N}_{S_{1}}\right]_{1} . \mathcal{B}_{S}$ works as follows: it takes input $\left(\rho^{\dagger}, h_{k t}\left(\theta^{\dagger}\right), \mathrm{crs}^{\dagger}\right)$ where

$\rho^{\dagger}:=\left(g k,\left[\mathbf{M}_{1, S_{0}}\right]_{1},\left[\mathbf{M}_{2, S_{0}}\right]_{2},\left[\mathbf{N}_{S_{1}}\right]_{1}\right), \quad h_{k t}\left(\theta^{\dagger}\right):=\mathbf{N}_{S_{1}}, \quad \operatorname{crs}^{\dagger}:=\left(\left[\mathbf{B}^{\dagger}\right]_{1},\left[\mathbf{D}^{\dagger}\right]_{2},[\mathbf{A}]_{1,2},\left[\mathbf{C}_{1}^{\dagger}\right]_{2},\left[\mathbf{C}_{2}^{\dagger}\right]_{1}\right)$ and does the following:

- It samples $\left(\left[\mathbf{Q}_{1}\right]_{1},\left[\mathbf{Q}_{2}\right]_{2}, \mathbf{Q}_{1}, \mathbf{Q}_{2}, \mathbf{T}_{\mathbf{Q}}\right) \leftarrow \mathrm{CS}^{\prime} \cdot \operatorname{KGen}\left(g k, d, K, S_{1}\right)$ and sets $\mathbf{Q}:=\mathbf{Q}_{1}+\mathbf{Q}_{2}$.

- It samples $\left([\mathbf{F}]_{1}, \mathbf{F}, \mathbf{T}_{\mathbf{F}}\right) \leftarrow$ CS.KGen $\left(g k, d, K, S_{2}\right)$.

- It samples $\mathbf{M}_{1, \bar{S}_{1}}, \mathbf{M}_{2, \bar{S}_{1}}, \mathbf{N}_{\bar{S}_{2}}$, such that $\mathbf{M}_{1}=\mathbf{P}_{S_{1}}\left(\begin{array}{l}\mathbf{M}_{1, S_{1}} \\ \mathbf{M}_{1, \bar{S}_{1}}\end{array}\right), \mathbf{M}_{2}=\mathbf{P}_{S_{1}}\left(\begin{array}{l}\mathbf{M}_{2, S_{1}} \\ \mathbf{M}_{2, \bar{S}_{1}}\end{array}\right), \mathbf{N}=\mathbf{P}_{S_{2}}\left(\begin{array}{l}\mathbf{N}_{S_{2}} \\ \mathbf{N}_{\bar{S}_{2}}\end{array}\right)$. 
- It samples $\mathbf{R}_{0} \leftarrow \mathbb{Z}_{p}^{\bar{K}_{0} \times k} ; \mathbf{R}_{1} \leftarrow \mathbb{Z}_{p}^{\bar{K}_{1} \times k}$

- It computes $[\mathbf{B}]_{1}:=\left[\mathbf{B}^{\dagger}\right]_{1}+\left[\mathbf{M}_{1}\right]_{1}^{\top} \mathbf{Q}^{\top} \mathbf{R}_{0}+[\mathbf{N}]_{1}^{\top} \mathbf{F}^{\top} \mathbf{R}_{1}$ and $[\mathbf{D}]_{2}:=\left[\mathbf{D}^{\dagger}\right]_{2}+\left[\mathbf{M}_{2}\right]_{2}^{\top} \mathbf{Q}^{\top} \mathbf{R}_{0}$

- It computes $\left[\mathbf{C}_{1}\right]_{2}:=\left(\begin{array}{cc}\mathbf{T}_{\mathbf{Q}} & \mathbf{0} \\ \mathbf{0} & \mathbf{T}_{\mathbf{F}}\end{array}\right)\left[\mathbf{C}_{1}^{\dagger}\right]_{2}+\left(\begin{array}{l}\mathbf{R}_{0} \\ \mathbf{R}_{1}\end{array}\right)[\mathbf{A}]_{2}$ and $\left[\mathbf{C}_{2}\right]_{1}:=\mathbf{T}_{\mathbf{Q}}\left[\mathbf{C}_{2}^{\dagger}\right]_{1}+\mathbf{R}_{0}[\mathbf{A}]_{1}$.

- It sets

$$
\begin{gathered}
\rho:=\left(\left[\mathbf{Q}_{1}\right]_{1},\left[\mathbf{Q}_{2}\right]_{1},[\mathbf{F}]_{2},\left[\mathbf{M}_{1}\right]_{1},\left[\mathbf{M}_{2}\right]_{2},[\mathbf{N}]_{1}\right), \quad h_{l s}(\theta):=\left(\mathbf{Q}_{1}, \mathbf{Q}_{2}, \mathbf{F}, \mathbf{N}\right) \\
\operatorname{crs}:=\left([\mathbf{B}]_{1},[\mathbf{D}]_{2},[\mathbf{A}]_{1,2},\left[\mathbf{C}_{1}\right]_{2},\left[\mathbf{C}_{2}\right]_{1}\right)
\end{gathered}
$$

It then executes $\mathcal{A}\left(\rho, h_{l s}(\theta)\right.$, crs $)$ until it outputs a statement $\left(\left[\boldsymbol{c}_{1}\right]_{1},\left[\boldsymbol{c}_{2}\right]_{2},[d]_{1}, \boldsymbol{w}\right)$ together with an accepting proof $[\pi]_{1},[\boldsymbol{\theta}]_{2}$. Given an accepting proof $\mathcal{B}$ sets $\left[x_{1}^{\dagger}\right]_{1}=\mathbf{T}_{\mathbf{Q}}\left[c_{1}\right]_{1},\left[x_{2}^{\dagger}\right]_{2}=$ $\mathbf{T}_{\mathbf{Q}}\left[\boldsymbol{c}_{2}\right]_{2},\left[\boldsymbol{y}^{\dagger}\right]_{1}=\mathbf{T}_{\mathbf{F}}[\boldsymbol{d}]_{1},\left[\boldsymbol{\pi}^{\dagger}\right]_{1}=[\boldsymbol{\pi}]_{1}-\left[\boldsymbol{c}_{1}\right]_{1}^{\top} \mathbf{R}_{1}-[\boldsymbol{d}]_{1}^{\top} \mathbf{R}_{2}$ and $\left[\boldsymbol{\theta}^{\dagger}\right]_{2}=[\boldsymbol{\theta}]_{1}-\left[\boldsymbol{c}_{2}\right]_{2}^{\top} \mathbf{R}_{1}$. It outputs $\left(\left(\left[x_{1}^{\dagger}\right]_{1},\left[x_{2}^{\dagger}\right]_{2},\left[y^{\dagger}\right]_{1}\right), \boldsymbol{w},\left(\left[\pi^{\dagger}\right]_{1},\left[\boldsymbol{\theta}^{\dagger}\right]_{2}\right)\right)$.

Note that by perfect completeness of the commitment scheme, the commitment keys are extractable and perfectly binding at $S$.

First, we claim that in this case the values $\rho, h_{l s}(\theta)$, crs output by $\mathcal{B}_{S}$ are identically distributed to honestly computed ones and thus do not skew the probability that $\mathcal{A}$ outputs a valid proof. For $\rho, h_{l s}(\theta)$, this is immediate by the witness samplability of the distributions $\mathcal{M}_{1}, \mathcal{M}_{2}, \mathcal{N}$. We show that this holds for crs as well. Let $\mathbf{K}_{0}^{\dagger} \in \mathbb{Z}_{p}^{\left|S_{1}\right| \times k}, \mathbf{K}_{1}^{\dagger} \in \mathbb{Z}_{p}^{\left|S_{2}\right| \times k}, \mathbf{Z}^{\dagger} \in \mathbb{Z}_{p}^{n \times k}$ matrices satisfying:

$$
\mathbf{B}^{\dagger}=\mathbf{M}_{1, S_{1}}^{\top} \mathbf{K}_{0}^{\dagger}+\mathbf{N}_{S}^{\top} \mathbf{K}_{1}^{\dagger}+\mathbf{Z}^{\dagger}, \mathbf{D}^{\dagger}=\mathbf{M}_{2, S_{1}}^{\top} \mathbf{K}_{0}^{\dagger}-\mathbf{Z}^{\dagger}, \mathbf{C}_{1}^{\dagger}=\left(\begin{array}{c}
\mathbf{K}_{0}^{\dagger} \\
\mathbf{K}_{1}^{\dagger}
\end{array}\right) \mathbf{A} \text { and } \mathbf{C}_{2}^{\dagger}=\mathbf{K}_{0}^{\dagger} \mathbf{A} \text {. }
$$

Now $\mathcal{B}_{S}$ implicitly defines $\mathbf{K}_{0}=\mathbf{T}_{\mathbf{Q}} \mathbf{K}_{0}^{\dagger}+\mathbf{R}_{0}, \mathbf{K}_{1}=\mathbf{T}_{\mathbf{F}} \mathbf{K}_{1}^{+}+\mathbf{R}_{1}$, and note that these matrices are uniformly distributed since $\mathbf{R}_{0}, \mathbf{R}_{1}$ are uniformly distributed. Thus $\mathbf{K}_{0}, \mathbf{K}_{1}$ are distributed identically to honestly generated values for generating a crs. We claim that the crs output by $\mathcal{A}$ is identically distributed to sampling this matrix and computing the other values honestly. Indeed we have that

$$
\begin{aligned}
\mathbf{B} & =\mathbf{B}^{\dagger}+\mathbf{M}_{1}^{\top} \mathbf{Q}^{\top} \mathbf{R}_{1}+\mathbf{N}_{1}^{\top} \mathbf{H}^{\top} \mathbf{R}_{1} \\
& =\mathbf{M}_{1, S_{1}}^{\top} \mathbf{K}_{0}^{\dagger}+\mathbf{N}_{S_{2}}^{\top} \mathbf{K}_{1}+\mathbf{Z}^{\dagger}+\mathbf{M}_{1}^{\top} \mathbf{Q}^{\top} \mathbf{R}_{0}+\mathbf{N}_{1}^{\top} \mathbf{H}^{\top} \mathbf{R}_{1} \\
& =\mathbf{M}_{1}^{\top} \mathbf{Q}^{\top} \mathbf{T}_{\mathbf{Q}} \mathbf{K}_{0}^{\dagger}+\mathbf{N}^{\top} \mathbf{F}^{\top} \mathbf{T}_{\mathbf{F}}^{\top} \mathbf{K}_{1}+\mathbf{Z}^{\dagger}+\mathbf{M}_{1}^{\top} \mathbf{Q}^{\top} \mathbf{R}_{0}+\mathbf{N}_{1}^{\top} \mathbf{H}^{\top} \mathbf{R}_{1} \\
& =\mathbf{M}_{1}^{\top} \mathbf{Q}^{\top}\left(\mathbf{T}_{\mathbf{Q}} \mathbf{K}_{0}^{\dagger}+\mathbf{R}_{0}\right)+\mathbf{N}^{\top} \mathbf{F}^{\top}\left(\mathbf{T}_{\mathbf{F}}^{\top} \mathbf{K}_{1}+\mathbf{R}_{1}\right)+\mathbf{Z}^{\dagger} \\
& =\mathbf{M}_{1}^{\top} \mathbf{Q}^{\top} \mathbf{K}_{0}+\mathbf{N}^{\top} \mathbf{F}^{\top} \mathbf{K}_{1}+\mathbf{Z}^{\dagger}
\end{aligned}
$$

where the third equality follows since by the local extractability of the SSBs (1) $\mathbf{T}_{\mathbf{Q}}^{\top} \mathbf{Q} \mathbf{M}_{1}=\mathbf{M}_{1, S}$ and (2) $\mathbf{T}_{\mathbf{F}}^{\top} \mathbf{F N}=\mathbf{N}_{S}$. Similarly, we have

$$
\begin{aligned}
\mathbf{D} & =\mathbf{D}^{\dagger}+\mathbf{M}_{2}^{\top} \mathbf{Q}^{\top} \mathbf{R}_{0} \\
& =\mathbf{M}_{2, S_{1}}^{\top} \mathbf{K}_{0}^{\dagger}-\mathbf{Z}^{\dagger}+\mathbf{M}_{2}^{\top} \mathbf{Q}^{\top} \mathbf{R}_{0} \\
& =\mathbf{M}_{2}^{\top} \mathbf{Q}^{\top} \mathbf{T}_{\mathbf{Q}} \mathbf{K}_{0}^{\dagger}-\mathbf{Z}^{\dagger}+\mathbf{M}_{2}^{\top} \mathbf{Q}^{\top} \mathbf{R}_{0} \\
& =\mathbf{M}_{2}^{\top} \mathbf{Q}^{\top}\left(\mathbf{T}_{\mathbf{Q}} \mathbf{K}_{0}^{\dagger}+\mathbf{R}_{0}\right)-\mathbf{Z}^{\dagger} \\
& =\mathbf{M}_{2}^{\top} \mathbf{Q}^{\top} \mathbf{K}_{0}-\mathbf{Z}^{\dagger}
\end{aligned}
$$


Also, we have that

$$
\begin{aligned}
& \mathbf{C}_{1}=\left(\begin{array}{cc}
\mathbf{T}_{\mathbf{Q}} & \mathbf{0} \\
\mathbf{0} & \mathbf{T}_{\mathbf{F}}
\end{array}\right) \mathbf{C}_{1}^{\dagger}+\left(\begin{array}{l}
\mathbf{R}_{0} \\
\mathbf{R}_{1}
\end{array}\right) \mathbf{A}=\left(\begin{array}{cc}
\mathbf{T}_{\mathbf{Q}} & \mathbf{0} \\
\mathbf{0} & \mathbf{T}_{\mathbf{F}}
\end{array}\right)\left(\begin{array}{l}
\mathbf{K}_{0}^{\dagger} \\
\mathbf{K}_{1}^{\dagger}
\end{array}\right) \mathbf{A}+\left(\begin{array}{l}
\mathbf{R}_{0} \\
\mathbf{R}_{1}
\end{array}\right) \mathbf{A}=i\left(\begin{array}{l}
\mathbf{T}_{\mathbf{Q}} \mathbf{K}_{0}^{\dagger}+\mathbf{R}_{0} \\
\mathbf{T}_{\mathbf{F}} \mathbf{K}_{1}^{\dagger}+\mathbf{R}_{1}
\end{array}\right) \mathbf{A}=\left(\begin{array}{l}
\mathbf{K}_{0} \\
\mathbf{K}_{1}
\end{array}\right) \mathbf{A} \\
& \mathbf{C}_{2}=\mathbf{T}_{\mathbf{Q}} \mathbf{C}_{2}^{\dagger}+\mathbf{R}_{0} \mathbf{A}=\mathbf{T}_{\mathbf{Q}} \mathbf{K}_{0}^{\dagger} \mathbf{A}+\mathbf{R}_{0} \mathbf{A}=\left(\mathbf{T}_{\mathbf{Q}} \mathbf{K}_{0}^{\dagger}+\mathbf{R}_{0}\right) \mathbf{A}=\mathbf{K}_{0} \mathbf{A}
\end{aligned}
$$

so the outputted crs is indeed identically distributed to an honest one.

Then, we show that $\mathcal{B}$ outputs a valid statement-proof pair w.r.t. to $\mathrm{crs}^{\dagger}$. Since the commitment keys are extractable and perfectly binding, we have that $\left(x_{1}^{\dagger}, x_{2}^{\dagger}\right)$ and $y^{\dagger}$ are valid openings for the commitments $\left(c_{1}, c_{2}\right)$ and $\boldsymbol{d}$ respectively. Assuming $\mathcal{A}$ produces a valid statement for $\mathcal{R}_{\rho, S^{\prime}}^{\text {no }}$ for the extracted values it holds that $x_{1}^{\dagger}+\boldsymbol{x}_{2}^{\dagger}=\left(\mathbf{M}_{1, S_{1}}+\mathbf{M}_{2, S_{1}}\right) \boldsymbol{w}$ and $\boldsymbol{y}^{\dagger} \neq \mathbf{N}_{S_{2}} \boldsymbol{w}$. Thus $\mathcal{B}_{S}$ outputs a valid statement and it suffices to show that $\left(\boldsymbol{\pi}^{\dagger}, \boldsymbol{\theta}^{\dagger}\right)$ is a valid proof. Indeed, we have

$$
\begin{aligned}
\mathbf{0}= & \pi \mathbf{A}+\boldsymbol{\theta} \mathbf{A}-\left(c_{1}^{\top} \mid \boldsymbol{d}^{\top}\right) \mathbf{C}_{1}-c_{2}^{\top} \mathbf{C}_{2} \\
= & \left(\pi^{\dagger}+c_{1}^{\top} \mathbf{R}_{0}+d^{\top} \mathbf{R}_{1}\right) \mathbf{A}+\left(\boldsymbol{\theta}^{\dagger}+c_{2}^{\top} \mathbf{R}_{0}\right) \mathbf{A} \\
& \quad-\left(c_{1}^{\top} \mid d^{\top}\right)\left(\left(\begin{array}{cc}
\mathbf{T}_{\mathbf{Q}} & \mathbf{0} \\
\mathbf{0} & \mathbf{T}_{\mathbf{F}}
\end{array}\right) \mathbf{C}_{1}^{\dagger}+\left(\begin{array}{l}
\mathbf{R}_{0} \\
\mathbf{R}_{1}
\end{array}\right) \mathbf{A}\right) \\
& \quad-c_{2}^{\top}\left(\mathbf{T}_{\mathbf{Q}} \mathbf{C}_{2}^{\dagger}+\mathbf{R}_{0} \mathbf{A}\right) \\
= & \left(\pi^{\dagger}+c_{1}^{\top} \mathbf{R}_{0}+d^{\top} \mathbf{R}_{1}\right) \mathbf{A}+\left(\boldsymbol{\theta}^{\dagger}+c_{2}^{\top} \mathbf{R}_{0}\right) \mathbf{A} \\
& \quad-\left(c_{1}^{\top} \mathbf{T}_{\mathbf{Q}} \mid \boldsymbol{d}^{\top} \mathbf{T}_{\mathbf{F}}\right) \mathbf{C}_{1}^{\dagger}-\left(c_{1}^{\top} \mathbf{R}_{0}-d^{\top} \mathbf{R}_{1}\right) \mathbf{A} \\
& \quad-c_{2}^{\top} \mathbf{T}_{\mathbf{Q}} \mathbf{C}_{2}^{\dagger}-c_{2}^{\top} \mathbf{R}_{0} \mathbf{A} \\
= & \pi^{\dagger} \mathbf{A}+\boldsymbol{\theta}^{\dagger} \mathbf{A}-\left(c_{1}^{\top} \mathbf{T}_{\mathbf{Q}} \mid d^{\top} \mathbf{T}_{\mathbf{F}}\right) \mathbf{C}_{1}^{\dagger}-c_{2}^{\top} \mathbf{T}_{\mathbf{Q}} \mathbf{C}_{2}^{\dagger} \\
= & \pi^{\dagger} \mathbf{A}+\boldsymbol{\theta}^{\dagger} \mathbf{A}-\left(x_{1}^{\dagger} \mid y^{\dagger^{\top}}\right) \mathbf{C}_{1}^{\dagger}-x_{2}^{\dagger^{\top}} \mathbf{C}_{2}^{\dagger}
\end{aligned}
$$

and the last equation is the verifying equation for the knowledge transfer argument for $\mathrm{crs}^{\dagger}$.

We next show that when the distributions $\left(\mathcal{M}_{1} \mathcal{M}_{2}\right), \mathcal{N}$ guarantee that the sum knowledge transfer argument is secure w.r.t. all possible sets $S$, construction QASum has $h_{l s}$-strong local knowledge soundness where $h_{l s}$ includes $\left.\mathbf{G}, \mathbf{H}, \mathbf{F},[\mathbf{M}]_{2},\left[\mathbf{N}_{1} \otimes \mathbf{N}_{2}-\mathbf{R}\right]_{1},[-\mathbf{R}]_{2}\right)$, for a uniform $\mathbf{R}$ and some extra information about the matrix $\mathbf{M}$.

Corollary 6. Let $\mathcal{D}_{k}$ be a matrix distribution for which $\mathcal{D}_{k}$-SKerMDH. Denote $\mathcal{M}_{1, S}$ (resp. $\mathcal{M}_{2, S}, \mathcal{N}_{S}$ ) the distributions that sample matrices from $\mathcal{M}_{1}\left(\right.$ res. $\left.\mathcal{M}_{2}, \mathcal{N}\right)$, and restricts them to rows corresponding to $S$. Then

1. If for all $S_{0} \subseteq[d]$ with $S_{0} \leq K_{0},\left(\mathcal{M}_{1, S_{0}}^{\top}, \mathcal{M}_{2, S_{0}}^{\top}, h\right)-\mathrm{MDDH}$ holds, QASum is an $h_{l s}$-strong local knowledge sound proof system, where $h_{l s}(\theta)=\left(h\left(\mathbf{M}_{1, S}, \mathbf{M}_{2, S}\right), \mathbf{G}, \mathbf{H}, \mathbf{F}, \mathbf{N}\right)$.

2. Iffor all $S_{0}, S_{1} \subseteq[d]$ with $S_{0} \leq K_{0}, S_{1} \leq K_{1}$ the distributions $\mathcal{M}_{1, S_{0}}, \mathcal{M}_{2, S_{0}}, \mathcal{N}_{S_{1}}$ output matrices with the last $n^{\prime}$ columns being $\mathbf{0}$, and $\left(\mathcal{M}_{1, S_{0}}^{\prime \top}, \mathcal{M}_{2, S_{0}}^{\prime \top}, h\right)-\mathrm{MDDH}$ holds, with $\mathcal{M}_{b, S_{0}}^{\prime}$ being $\mathcal{M}_{b, S_{0}}$ where we delete the trailing zero columns, then QASum is an $h_{l s}$-strong local knowledge sound proof system, where $h_{l s}(\theta)=\left(h\left(\mathbf{M}_{1, S_{0}}, \mathbf{M}_{2, S_{0}}\right), \mathbf{G}, \mathbf{H}, \mathbf{F}, \mathbf{N}\right)$.

Proof. The proof is an immediate consequence of Thm. 12 and Thm. 19.1 for case 1 and Thm. 19.2 for case 2. 
The proof that QASum is oblivious follows from the oblivious trapdoor generation and index set hiding of SSB commitments. We follow essentially the same proof as in the QABlin case.

First we show the corresponding lemma to Lemma 2, that is, we construct an indistinguishable crs given only the commitment keys and the matrices $\mathbf{M}_{1}, \mathbf{M}_{2}, \mathbf{N}$.

Lemma 3. There exists a modified crs generation algorithm $\mathrm{K}^{\prime}$ that on input $\left(\rho, \theta^{\prime}\right)$, where $\theta^{\prime}$ contains only either $\mathbf{M}_{1}, \mathbf{M}_{2}, \mathbf{N}$ or $\mathbf{Q}_{1}, \mathbf{Q}_{2}, \mathbf{F}$ and outputs a crs such that $(\rho, \mathrm{crs})$ are identically distributed to the honest algorithm.

Proof. Given these values we can compute the crs using a simple trick. Instead of computing

$$
\begin{gathered}
{[\mathbf{B}]_{1}=\left[\mathbf{M}_{1}^{\top}\right]_{1} \mathbf{Q}^{\top} \mathbf{K}_{0}+\left[\mathbf{N}^{\top}\right]_{1} \mathbf{F}^{\top} \mathbf{K}_{1}+[\mathbf{Z}]_{1}} \\
{[\mathbf{D}]_{2}=\left[\mathbf{M}_{2}^{\top}\right]_{2} \mathbf{Q}^{\top} \mathbf{K}_{0}-[\mathbf{Z}]_{2},}
\end{gathered}
$$

we compute

$$
\begin{gathered}
{[\mathbf{B}]_{1}=\left(\mathbf{M}_{1}^{\top}+\mathbf{M}_{2}^{\top}\right)\left[\mathbf{Q}_{1}^{\top}\right]_{1} \mathbf{K}_{0}+\left[\mathbf{N}^{\top}\right]_{1} \mathbf{F}^{\top} \mathbf{K}_{1}+[\mathbf{Z}]_{1}} \\
{[\mathbf{D}]_{2}=\left(\mathbf{M}_{2}^{\top}+\mathbf{M}_{2}^{\top}\right)\left[\mathbf{Q}_{2}^{\top}\right]_{2} \mathbf{K}_{0}-[\mathbf{Z}]_{2},}
\end{gathered}
$$

Noting that in both cases the elements computed are uniformly distributed conditioned on $\mathbf{B}+\mathbf{D}=\left(\mathbf{M}_{1}^{\top}+\mathbf{M}_{2}^{\top}\right)\left(\mathbf{Q}_{1}^{\top}+\mathbf{Q}_{2}^{\top}\right) \mathbf{K}_{0}+\mathbf{N}^{\top} \mathbf{F}^{\top} \mathbf{K}_{1}$ we see that these values are computed as in the honest setup.

In the case where $\theta=\left(\mathbf{Q}_{1}, \mathbf{Q}_{2}, \mathbf{F}\right)$ we can directly compute the crs by noting that $\mathbf{Q}=\mathbf{Q}_{1}+\mathbf{Q}_{2}$ and the group elements in $\rho$ are enough to compute all values of crs.

As in the previous cases, we abuse notation and refer to $\mathrm{K}^{\prime}\left(\rho, \theta^{\prime}\right)$ as $\mathrm{K}\left(\rho, \theta^{\prime}\right)$.

The proof of oblivious extraction essentially follows from the oblivious key generation and index set hiding of the SSB commitments and is similar to the proof of Thm. 11 .

Theorem 13. Let $\mathcal{M}_{1}, \mathcal{M}_{2}$ be (possibly correlated) witness samplable distribution, $\mathcal{N}$ be a witness samplable distribution, and CS, CS' be an algebraic and a split algebraic SSB commitment scheme respectively with perfect completeness, oblivious trapdoor generation and $h, h^{\prime}$-index set hiding respectively. Then

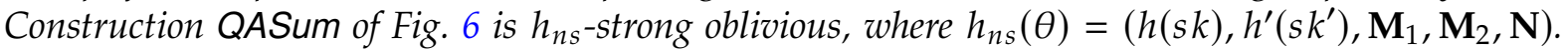
Furthermore,

1. For every PPT $\mathcal{A}$ against index set hiding of $Q A S$ Sum, there exist adversaries $\mathcal{B}_{0}, \mathcal{B}_{1}$ against index set hiding property of $\mathrm{CS}^{\prime}$, CS respectively, such that $\operatorname{Adv}_{\mathrm{ISH}}^{Q A S u m}(\mathcal{A}) \leq \operatorname{Adv}_{\mathrm{ISH}}^{\mathrm{CS}}\left(\mathcal{B}_{0}\right)+\operatorname{Adv}_{\mathrm{ISH}}^{\mathrm{CS}}\left(\mathcal{B}_{1}\right)$.

2. For every $\mathcal{A}$ against oblivious crs generation of $Q A S u m$, there exist an adversaries $\mathcal{B}_{0}, \mathcal{B}_{1}$ against oblivious key generation of $\mathrm{CS}^{\prime}, \mathrm{CS}$ respectively, such that $\operatorname{Adv}_{\mathrm{oblv}}^{Q A S u m}(\mathcal{A}) \leq \operatorname{Adv}_{\mathrm{oblv}}^{\mathrm{CS}}\left(\mathcal{B}_{0}\right)+$ $\operatorname{Adv}_{\text {oblv }}^{\mathrm{CS}}\left(\mathcal{B}_{1}\right)$.

Proof. It is enough to show that $h_{n s}$-strong index set hiding holds and that we can sample a tuple $(\rho, \mathrm{crs})$ indistinguishable from the one we are given, along with a valid trapdoor. This is the case because the commitment keys are perfectly binding in $S^{\prime}$, which means that the witnesses are unique and do not help the (unbounded) distinguisher who can compute them on its own.

Index Set Hidning. Assume there exist sets $S, S^{\prime}$ of size at most $K$ and an adversary $\mathcal{A}$ which distinguishes $\left(\rho\right.$, crs) sampled for $S$ from $(\rho$, crs $)$ sampled for $S^{\prime}$ with some probability $\alpha$. We construct adversaries $\mathcal{B}_{0}$ distinguishing $c k_{0}$ sampled for $S_{1}$ from $c k_{0}$ sampled for $S_{1}^{\prime}$ with probability $\alpha_{0}$ and an adversary $\mathcal{B}_{1}$ distinguishing $c k_{1}$ sampled for $S_{2}$ from $c k_{1}$ sampled for $S_{2}^{\prime}$ with probability $\alpha_{1}$ such that $\alpha \leq \frac{\alpha_{0}+\alpha_{1}}{2}$. 
$\mathcal{B}_{0}$ takes as input some $c k_{0}$ and $h^{\prime}\left(\mathbf{s k}_{0}\right)$ sampled either for $S_{0}$ or $S_{0}^{\prime}$ and parses $c k_{0}$ as $[\mathbf{Q}]_{1},[\mathbf{Q}]_{2}$, aux. It then honestly computes the crs by sampling $\mathbf{M}_{1}, \mathbf{M}_{2}, \mathbf{N}$ and following the $\mathrm{K}$ described in Lemma 3 except that $c k_{1}$ is computed as follows: it samples $b \leftarrow\{0,1\}$ and if $b=0$ it sets $\left(c k_{1}, s k_{1}\right) \leftarrow \operatorname{CS}$.KeyGen $\left(g k_{1}, d, K, S_{1}\right)$ otherwise it sets $\left(c k_{1}, s k_{1}\right) \leftarrow$ CS.KeyGen $\left(g k_{1}, d, K, S_{1}^{\prime}\right)$.Note that, with probability $1 / 2$, the crs computed by $\mathcal{B}$ follows exactly the original distribution. This is the case since $\mathbf{B}, \mathbf{D}$ are uniform matrices conditioned on their sum being equal to $\left(\mathbf{M}_{1}^{\top}+\mathbf{M}_{2}^{\top}\right)\left(\mathbf{Q}_{1}^{\top}+\mathbf{Q}_{2}^{\top}\right) \mathbf{K}_{1}+\mathbf{N}^{\top} \mathbf{F}^{\top} \mathbf{K}_{2}$ for uniform $\mathbf{K}_{1}, \mathbf{K}_{2}$, exactly as in the honest crs generation. Finally $\mathcal{B}_{0}$ runs $\mathcal{A}\left(\rho, \mathrm{crs}, h_{n s}(\theta)=\left(h^{\prime}\left(\mathrm{sk}_{0}\right), h^{\prime}\left(\mathrm{sk}_{1}\right), \mathbf{M}_{1}, \mathbf{M}_{2}, \mathbf{N}\right)\right)$ and output whatever it outputs.

Similarly, on input $c k_{1}, h\left(\mathbf{s k}_{1}\right)$ sampled either for $S_{1}$ or $S_{1}^{\prime}, \mathcal{B}_{1}$ samples $b \leftarrow\{0,1\}$ and if $b=0$ it sets $\left(c k_{0}, s k_{0}\right) \leftarrow$ CS.KeyGen $\left(g k, d, K, S_{0}\right)$ otherwise it sets $\left(c k_{0}, s k_{0}\right) \leftarrow$ CS.KeyGen $\left(g k, d, K, S_{0}^{\prime}\right)$ and honestly computes the crs as in the previous case. A simple case analysis shows that $\rho \leq \frac{\rho_{1}+\rho_{2}}{2}$.

Oblivious trapdoor generation: We show how to obliviously sample a trapdoor given black box access to CS.OblKeyGen and CS'.OblKeyGen. For oblivious trapdoor generation, given a pair $\rho$, crs for the quasi argument and set $S^{\prime}$ the oblivious setup QASum.OblKeyGen does the following:

- $\left(c k_{0}^{\prime}, \tau_{0}^{\prime}\right) \leftarrow$ CS.OblKeyGen $\left(c k_{0}, S_{0}^{\prime}\right)$ and $\left(c k_{1}^{\prime}, \tau_{1}^{\prime}\right) \leftarrow$ CS.OblKeyGen $\left(c k_{1}, S_{1}^{\prime}\right)$.

- Sample $\left(\left[\mathbf{M}_{1}\right]_{1},\left[\mathbf{M}_{2}\right]_{2}, \mathbf{M}_{1}, \mathbf{M}_{2}\right) \leftarrow \mathcal{M},\left([\mathbf{N}]_{1}, \mathbf{N}\right) \leftarrow \mathcal{N}$.

- Compute the rest of the crs by $\mathrm{K}\left(c k_{0}^{\prime}, c k_{1}^{\prime}, \mathbf{M}_{1}, \mathbf{M}_{2}, \mathbf{N}\right)$.

Arguing as in the index set hiding proof, the only difference in the oblivious and an honest crs is how the commitment keys are sampled. We can thus use a standard hybrid argument to reduce the property to the oblivious trapdoor generation of the commitment schemes CS, $\mathrm{CS}^{\prime}$.

Corollary 7. If CS is the one from fig. 3, and CS is the construction of kCS of Thm. 6, then QASum from fig. 6 is $h_{n s}$-strong no-signaling where $h_{n s}(\theta)=\left(\mathbf{M}, \mathbf{N}_{1}, \mathbf{N}_{2}\right)$.

Proof. The proof follows directly from Theorem 7 and the $h_{n s}$-strong oblivious property of QASum, which in turn follows from applying Theorems 2, 6 to Theorem 13. 


\subsubsection{Quasi-Arguments for Hadamard Products.}

The main result of [GHR15b] was implicitly a quasi-argument for the set of equations $b_{i}\left(b_{i}-1\right)=$ 0 , for all $i \in[d]$. We extend their results to equations of the form $x_{i} y_{i}=z_{i}$, that is $\boldsymbol{x} \circ \boldsymbol{y}=\boldsymbol{z}$ where $\circ$ denotes the hadamard product. Let $\mathcal{U}, \mathcal{V}, \mathcal{W}$ be witness samplable distributions over matrices in $\mathbb{G}_{1}^{d \times n}, \mathbb{G}_{2}^{d \times n}$ and $\mathbb{G}_{1}^{d \times n}$, respectively, for $n, d \in \mathbb{N}$. Let $K=(K, K)$ with $K \leq d$ and $S=(S, S)$ with $S \subseteq[d]$ and $S \leq K$. Also let $C S$ be an algebraic SSB commitment scheme with commitment space $\mathbb{G}_{\mu}^{\bar{K}}$. The parameter language is

$$
\begin{aligned}
\mathcal{L}_{\text {par }}=\left\{[\mathbf{U}]_{1},\right. & {[\mathbf{V}]_{2},[\mathbf{W}]_{1},[\mathbf{G}]_{1},[\mathbf{H}]_{2},[\mathbf{F}]_{1} \mid \exists \mathbf{U}, \mathbf{V}, \mathbf{W}, \mathbf{G}, \mathbf{H}, \mathbf{F} \text { s.t. } } \\
& \left([\mathbf{U}]_{1}, \mathbf{U}\right) \in \operatorname{Sup}(\mathcal{U}),\left([\mathbf{V}]_{2}, \mathbf{V}\right) \in \operatorname{Sup}(\mathcal{V}),\left([\mathbf{W}]_{1}, \mathbf{W}\right) \in \operatorname{Sup}(\mathcal{W}), \\
& \left([\mathbf{G}]_{1}, \mathbf{G}, \mathbf{T}_{\mathbf{G}}\right) \in \operatorname{Sup}\left(C S . K e y G e n\left(g k_{1}, n, K, S\right)\right) \\
& \left([\mathbf{H}]_{2}, \mathbf{H}, \mathbf{T}_{\mathbf{H}}\right) \in \operatorname{Sup}\left(\text { CS.KeyGen }\left(g k_{2}, n, K, S\right)\right) \\
& \left.\left([\mathbf{F}]_{1}, \mathbf{F}, \mathbf{T}_{\mathbf{F}}\right) \in \operatorname{Sup}\left(\text { CS.KeyGen }\left(g k_{1}, n, K, S\right)\right)\right\}
\end{aligned}
$$

We assume w.l.o.g. that the corresponding relation is efficiently verifiable. The parameters $\rho=\left([\mathbf{U}]_{1},[\mathbf{V}]_{2},[\mathbf{W}]_{1},[\mathbf{G}]_{1},[\mathbf{H}]_{2},[\mathbf{F}]_{1}\right)$ define the following relations:

$$
\begin{aligned}
& \mathcal{R}_{\rho}^{\text {yes }}=\left\{\begin{array}{l|l}
{[u]_{1},[v]_{2},[\boldsymbol{w}]_{2}, \boldsymbol{a}, \boldsymbol{b}} & \begin{array}{c}
\boldsymbol{u}=\mathrm{GU} \boldsymbol{a}, \boldsymbol{v}=\mathbf{H V} \boldsymbol{b} \\
\boldsymbol{w}=\mathrm{FW}(\boldsymbol{a} \circ \boldsymbol{b})
\end{array}
\end{array}\right\}, \\
& \mathcal{R}_{\rho, S}^{\text {no }}=\left\{\begin{array}{c|c}
\left([\boldsymbol{v}]_{1},[\boldsymbol{u}]_{2},[\boldsymbol{w}]_{1}\right), \boldsymbol{a}, \boldsymbol{b} & \begin{array}{c}
\boldsymbol{x}_{1}, \boldsymbol{x}_{2}, \boldsymbol{y} \text { are valid } S \text { openings of } \\
\left(\left[\boldsymbol{x}_{1}\right]_{1},\left[\boldsymbol{x}_{2}\right]_{2},[\boldsymbol{y}]_{1}\right)
\end{array} \\
\begin{array}{c}
\boldsymbol{c}_{1}, \boldsymbol{c}_{2}, \boldsymbol{d} \text { w.r.t. } \mathbf{G}, \mathbf{H}, \mathbf{F} \text { respectively and } \\
\boldsymbol{x}_{1}=\mathbf{U}_{S} \boldsymbol{a}, \boldsymbol{x}_{2}=\mathbf{V}_{S} \boldsymbol{b}, \text { but } \boldsymbol{y} \neq \mathbf{W}_{S}(\boldsymbol{a} \circ \boldsymbol{b})
\end{array}
\end{array}\right\} .
\end{aligned}
$$

That is the partial witness for $S$ is some valid local openings $\left[x_{1}\right]_{1},\left[x_{2}\right]_{2},[y]_{1}$ w.r.t. to $\mathbf{G}, \mathbf{H}, \mathbf{F}$ respectively that satisfy the following: if $x_{1}=\mathbf{U}_{S} \boldsymbol{a}$ and $x_{2}=\mathbf{V}_{S} \boldsymbol{b}$ and then it should be the case that $\boldsymbol{y}=\mathbf{W}_{S} \boldsymbol{c}$ where $\boldsymbol{c}=\boldsymbol{a} \circ \boldsymbol{b}$. Here $\boldsymbol{a}, \boldsymbol{b}$ is the promise of the adversary. We present the protocol in Fig 7. Essentially, we first have the prover commit to the kronecker product $\boldsymbol{a} \otimes \boldsymbol{b}$ using a commitment scheme defined by the $\otimes$ operation of CS to itself, and then show that if the split opening of this commitment is $w=a \otimes b$, then the opening of $\boldsymbol{d}$ is $\mathrm{D} w$ where $\mathrm{D}$ is the linear operation that outputs $\boldsymbol{a} \circ \boldsymbol{b}$ on input $\boldsymbol{a} \otimes \boldsymbol{b}$. The former "promise", regarding the kronecker product, is verified by the pairing operation, while for the latter construction QASum is used.

Theorem 14. Let $\mathcal{U}, \mathcal{V}, \mathcal{W}$ be witness samplable distributions, $\mathcal{D}_{k}$ be a matrix distribution and $\mathrm{CS}$ an algebraic SSB commitment scheme with perfect completeness. Also, let $\mathcal{A}$ be an adversary against $h_{l s}$-strong local knowledge soundness of QAHad where $h_{l s}(\theta)=\left(\mathbf{G}, \mathbf{H}, \mathbf{F}, \mathbf{W},[\mathbf{U} \otimes \mathbf{V}-\mathbf{R}]_{1},[\mathbf{R}]_{2}\right)$ for a uniformly distributed $\mathbf{R}$. Then completeness holds with probability 1 and for $h_{l s}$-strong local soundness it holds that $\operatorname{Adv}_{\text {snd }}^{Q A H a d}(\mathcal{A}) \leq \operatorname{Adv}_{\text {snd }}^{Q A S u m}(\mathcal{B})$ where $\mathcal{B}$ is an adversary against $h_{\text {ls-sum-strong }}$ local soundness of QASum for $\rho_{\text {sum }}$ as computed in Fig. 7 and $h_{l s-s u m}\left(\theta_{\text {sum }}\right)$ outputs $\theta_{\text {sum }}$ except the matrices $\mathbf{M}_{1}, \mathbf{M}_{2}$.

Proof. For completeness, we have that

$$
\begin{aligned}
\boldsymbol{u} \otimes \boldsymbol{v} & =\mathbf{G U} \boldsymbol{a} \otimes \mathbf{G U} \boldsymbol{b}=(\mathbf{G} \otimes \mathbf{H})(\mathbf{U} \otimes \mathbf{V})(\boldsymbol{a} \otimes \boldsymbol{b})= \\
& =(\mathbf{G} \otimes \mathbf{H}-\mathbf{Z}+\mathbf{Z})(\mathbf{U} \otimes \mathbf{V}-\mathbf{R}+\mathbf{R})(\boldsymbol{a} \otimes \boldsymbol{b})= \\
& =\left(\mathbf{Q}_{1}+\mathbf{Q}_{2}\right)\left(\mathbf{M}_{1}+\mathbf{M}_{2}\right)(\boldsymbol{a} \otimes \boldsymbol{b})
\end{aligned}
$$

and also $c_{1}+c_{2}=\left(\mathbf{E}_{1}+\mathbf{E}_{2}\right)(\boldsymbol{a} \otimes \boldsymbol{b})=\left(\mathbf{Q}_{1}+\mathbf{Q}_{2}\right)(\mathbf{U} \otimes \mathbf{V})(\boldsymbol{a} \otimes \boldsymbol{b})=\boldsymbol{u} \otimes \boldsymbol{v}$, so the pairing test is successful. Finally, noting that $\boldsymbol{w}=\boldsymbol{d}=\mathrm{FW}(\boldsymbol{a} \circ \boldsymbol{b})=\mathrm{FWD}(\boldsymbol{a} \otimes \boldsymbol{b})=\mathrm{FN}(\boldsymbol{a} \otimes \boldsymbol{b})$, we see that 
the statement/witness pair $\left(\left[\boldsymbol{c}_{1}\right]_{1},\left[\boldsymbol{c}_{2}\right]_{2},[\boldsymbol{d}]_{1}\right), \boldsymbol{a} \otimes \boldsymbol{b}$ is a yes instance of the sum language for parameters $\rho_{\text {sum }}$ and the second condition for verification follows by the completeness of the QASum.

For local knowledge soundness, it is enough to note that the Kronecker part of the knowledge transfer holds unconditionally, that is, if for some promise $\boldsymbol{a}, \boldsymbol{b}$ it holds that $\boldsymbol{u}=\mathrm{GU} \boldsymbol{a}$ and $v=\mathbf{H V} \boldsymbol{b}$, then by the verification of the pairing condition, $\boldsymbol{c}_{1}+\boldsymbol{c}_{2}=\left(\mathbf{Q}_{1}+\mathbf{Q}_{2}\right)\left(\mathbf{M}_{1}+\mathbf{M}_{2}\right)(\boldsymbol{a} \otimes \boldsymbol{b})$, so we efficiently construct a promise for the sum language. Also, the value $h_{1 s-s u m}\left(\theta_{\text {sum }}\right)$ can be computed given $h_{l s}(\theta)$. Now, an accepting proof for the hadamard language contains an accepting proof for the sum language and we use that to break $q$-strong local soundness of QASum. Details follow.

Let $\mathcal{A}$ be an adversary against $h_{l s}$-strong local knowledge soundness of QAHad. We con-

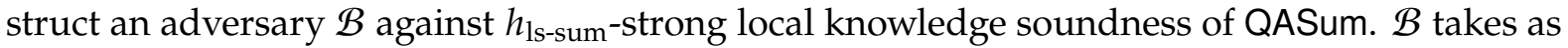
input $\left(\rho_{\text {sum }}, h_{\text {ls-sum }}\left(\theta_{\text {sum }}\right)\right.$, crs $\left._{\text {sum }}\right)$ and works as follows:

- Parse

$$
\begin{gathered}
\rho_{\text {sum }}=\left(g k,\left[\mathbf{Q}_{1}\right]_{1},\left[\mathbf{Q}_{2}\right]_{2},[\mathbf{F}]_{1},\left[\mathbf{M}_{1}\right]_{1},\left[\mathbf{M}_{2}\right]_{2},[\mathbf{N}]_{1}, \operatorname{aux} \mathrm{CS}=(\mathbf{G}, \mathbf{H}), \operatorname{aux}_{\mathcal{M}}=\left([\mathbf{U}]_{1},[\mathbf{V}]_{2}\right),\right. \\
\theta_{q_{\text {sum }}}=\left(\mathbf{Q}_{1}, \mathbf{Q}_{2}, \mathbf{G}, \mathbf{H}, \mathbf{F}, \mathbf{N}\right)
\end{gathered}
$$

- Set $\rho=\left(g k,[\mathbf{G}]_{1},[\mathbf{H}]_{2},[\mathbf{F}]_{2},[\mathbf{U}]_{1},[\mathbf{V}]_{2},[\mathbf{N}]_{1}\right), h_{l s}(\theta)=\left(\mathbf{G}, \mathbf{H}, \mathbf{F}, \mathbf{N},\left[\mathbf{M}_{1}\right]_{1},\left[\mathbf{M}_{2}\right]_{2}\right)$.

- It samples $\mathbf{R}^{\prime} \leftarrow \mathbb{Z}_{p}^{\bar{K}} \times n^{2}$ and sets $\left[\mathbf{E}_{1}\right]_{1}=\left(\mathbf{Q}_{1}+\mathbf{Q}_{2}\right)\left[\mathbf{M}_{1}\right]_{1}+\left[\mathbf{R}^{\prime}\right]_{1},\left[\mathbf{E}_{2}\right]_{2}=\left(\mathbf{Q}_{1}+\mathbf{Q}_{2}\right)\left[\mathbf{M}_{2}\right]_{2}-$ $\left[\mathbf{R}^{\prime}\right]_{2}$.

It then executes $\mathcal{A}\left(\rho, h_{l s}(\theta), \operatorname{crs}=\left(\left[\mathbf{E}_{1}\right]_{1},\left[\mathbf{E}_{2}\right]_{2}, \mathrm{crs}_{\text {sum }}\right)\right)$ until it outputs a statement $\left([u]_{1},[v]_{2}\right.$, $\left.[\boldsymbol{w}]_{1}, \boldsymbol{a}, \boldsymbol{b}\right)$ together with an accepting proof $\left(\left[\boldsymbol{c}_{1}\right]_{1},\left[\boldsymbol{c}_{2}\right]_{2}, \pi_{\text {sum }}\right)$. It outputs the statement/advice/proof tuple

$$
\left(\left(\left[\boldsymbol{c}_{1}\right]_{1},\left[\boldsymbol{c}_{2}\right]_{2},[\boldsymbol{w}]_{1}\right), \boldsymbol{a} \otimes \boldsymbol{b}, \pi_{\mathrm{sum}}\right) .
$$

The crs is identically distributed to an honestly computed one. Indeed the only thing computed differently are the values $\left[\mathbf{E}_{1}\right]_{1},\left[\mathbf{E}_{2}\right]_{2}$, but note that in the reduction they are distributed uniformly conditioned on $\mathbf{E}_{1}+\mathbf{E}_{2}=\left(\mathbf{Q}_{1}+\mathbf{Q}_{2}\right)\left(\mathbf{M}_{1}+\mathbf{M}_{2}\right)=\left(\mathbf{Q}_{1}+\mathbf{Q}_{2}\right)(\mathbf{U} \otimes \mathbf{V})$, as in the honest crs generation.

Now, assuming an accepting proof, and a correct promise $\boldsymbol{a}, \boldsymbol{b}$ given from $\mathcal{A}$ means that the promise of $\mathcal{B}$ is also correct. Indeed, we have

$$
\begin{aligned}
c_{1}+c_{2} & =\boldsymbol{u} \otimes v=\mathbf{G U} \boldsymbol{a} \otimes \mathbf{H V} \boldsymbol{b}=(\mathbf{G} \otimes \mathbf{H})(\mathbf{U} \otimes \mathbf{V})(\boldsymbol{a} \otimes \boldsymbol{b})= \\
& =(\mathbf{G} \otimes \mathbf{H}-\mathbf{Z}+\mathbf{Z})(\mathbf{U} \otimes \mathbf{V}-\mathbf{R}+\mathbf{R})(\boldsymbol{a} \otimes \boldsymbol{b})= \\
& =\left(\mathbf{Q}_{1}+\mathbf{Q}_{2}\right)\left(\mathbf{M}_{1}+\mathbf{M}_{2}\right)(\boldsymbol{a} \otimes \boldsymbol{b}) .
\end{aligned}
$$

Now let $x_{1}=\mathrm{T}_{\mathrm{Q}} c_{1}, x_{2}=\mathrm{T}_{\mathrm{Q}} c_{2}, y=\mathrm{T}_{\mathrm{F}} w$ be the extracted values. We have that

$$
\begin{aligned}
x_{1}+x_{2} & =\mathbf{T}_{\mathbf{Q}}\left(c_{1}+c_{2}\right)=\mathbf{T}_{\mathbf{Q}}\left(\mathbf{Q}_{1}+\mathbf{Q}_{2}\right)\left(\mathbf{M}_{1}+\mathbf{M}_{2}\right)(\boldsymbol{a} \otimes \boldsymbol{b}) \\
& =\left(\mathbf{M}_{S, 1}+\mathbf{M}_{S, 2}\right)(\boldsymbol{a} \otimes \boldsymbol{b}) .
\end{aligned}
$$

so indeed the promise is correct. Also assuming that the statement/advice given from $\mathcal{A}$ is a no-instance for the hadamard language w.r.t. to the set $S$, then the statement/advice given from $\mathcal{B}$ is a no-instance for the sum language w.r.t. the same set $S$. Indeed, we have

$$
y \neq \mathbf{W}_{S}(\boldsymbol{a} \circ \boldsymbol{b})=\mathrm{W}_{S} \mathrm{D}(\boldsymbol{a} \otimes \boldsymbol{b})=\mathrm{N}_{S}(\boldsymbol{a} \otimes \boldsymbol{b}) .
$$

So, conditioned on a successful $\mathcal{A}, \mathcal{B}$ outputs an instance/advice such that (1) the extractor gets values that satisfy $\mathcal{R}_{\rho_{\text {sum }, S}}^{\text {no }}$ for $\rho_{\text {sum }}$ and (2) a proof that verifies w.r.t. the instance. 
We next show that when the distributions $\mathcal{U}, \mathcal{V}, \mathcal{W}$ guarantee that the sum knowledge transfer argument is secure w.r.t. all possible sets $S$, construction QAHad has $h_{l s}$-strong local knowledge soundness where $h_{l s}$ includes $\mathbf{G}, \mathbf{H}, \mathbf{F}, \mathbf{W},[\mathbf{U} \otimes \mathbf{V}-\mathbf{R}]_{1},[\mathbf{R}]_{2}$ for a uniform $\mathbf{R}$.

Corollary 8. Let $\mathcal{D}_{k}$ be a matrix distribution for which $\mathcal{D}_{k}$-SKerMDH and let $D D H$ hold in $\mathbb{G}_{1}, \mathbb{G}_{2}$. Denote $\mathcal{U}_{S}\left(\right.$ resp. $\left.\mathcal{V}_{S}, \mathcal{W}_{S}\right)$ the distributions that sample matrices from $\mathcal{U}$ (res. $\mathcal{V}_{2}, \mathcal{W}$ ), and restricts them to rows corresponding to $S$. Then

1. If for all $S \subseteq[d]$ with $S \leq K_{0}, \mathcal{U}_{S}^{\top}-\mathrm{MDDH}$ and $\mathcal{V}_{S}^{\top}-\mathrm{MDDH}$ hold, $Q A H a d$ is an $h_{l s}$-strong local knowledge sound proof system, where $h_{l s}(\theta)=\left(\mathbf{G}, \mathbf{H}, \mathbf{F}, \mathbf{N},[\mathbf{U} \otimes \mathbf{V}-\mathbf{R}]_{1},[\mathbf{R}]_{2}\right)$ for a uniform $\mathbf{R}$.

2. If for all $S, S \subseteq[d]$ with $S \leq K$ the distributions $\mathcal{U}_{S}, \mathcal{V}_{S}, \mathcal{W}_{S}$ output matrices with the last $n^{\prime}$ columns being $\mathbf{0}$, and $\mathcal{U}_{S}^{\prime \top}-\mathrm{MDDH}$ and $\mathcal{V}_{S}^{\prime \top}-\mathrm{MDDH}$ hold, with $\mathcal{U}^{\prime}{ }_{S}$, (resp. $\mathcal{V}^{\prime}{ }_{S}$ ) being $\mathcal{U}_{S}$ (resp. $\left.\mathcal{V}_{S}\right)$ where we delete the trailing zero columns, then QAHad is an $h_{l s}$-strong local knowledge sound proof system, where $h_{l s}(\theta)=\left(\mathbf{G}, \mathbf{H}, \mathbf{F}, \mathbf{W},[\mathbf{U} \otimes \mathbf{V}-\mathbf{R}]_{1},[\mathbf{R}]_{2}\right)$.

Proof. By Thm. 14 it is enough to show that QASum is secure for such distribution. This in turn hold when the sum knowledge transfer argument is sound (Thm. 12) which is true if $\mathcal{D}_{k^{-}}$ SKerMDH holds and $\left(\mathcal{U}_{S}, \mathcal{V}_{S}, h\right)-\mathrm{MDDH}$ assumption holds (similar in the second case for the distributions we remove the zeros) by Thm. 19. It remains to show that for these distribution the latter condition holds when we are given the extra information $h(\mathbf{U}, \mathbf{V})=\left([\mathbf{U} \otimes \mathbf{V}-\mathbf{R}]_{1},[\mathbf{R}]_{2}\right)$ for a uniform $\mathbf{R}$. We show that this is the case if, additionally, DDH hold. That is we need to show that for all $S$ the $\left(\mathcal{U}_{S}, \mathcal{V}_{S}, h\right)$-MDDH holds or equivalently the distributions

- $\left[\mathbf{U}^{\top}\right]_{1},\left[\mathbf{V}^{\top}\right]_{2},\left[\mathbf{U}^{\top} \otimes \mathbf{V}^{\top}-\mathbf{R}\right]_{1},[\mathbf{R}]_{2},\left[(\mathbf{U} \otimes \mathbf{V})^{\top} \boldsymbol{k}-\boldsymbol{r}\right]_{1},[\boldsymbol{r}]_{2}: \boldsymbol{k} \leftarrow \mathbb{Z}_{q}^{|S|^{2}} ; \boldsymbol{r} \leftarrow \mathbb{Z}_{q}^{n^{2}} ; k \leftarrow \mathbb{Z}_{q}$

- $\left[\mathbf{U}^{\top}\right]_{1},\left[\mathbf{V}^{\top}\right]_{2},\left[\mathbf{U}^{\top} \otimes \mathbf{V}^{\top}-\mathbf{R}\right]_{1},[\mathbf{R}]_{2},[s]_{1},[\boldsymbol{t}]_{2}: s, t \leftarrow \mathbb{Z}_{q}^{n^{2}}$

where $\mathbf{U} \leftarrow \mathcal{U}_{S} ; \mathbf{V} \leftarrow \mathcal{V}_{S} ; \mathbf{R} \leftarrow \mathbb{Z}_{q}^{n^{2} \times|S|^{2}}$ are computationally indistuinguishable.

Let $S \subseteq[d]$ with $|S| \leq K$. We show the indistinguishability of these distributions by showing indistinguishability of a sequence of hybrid distributions. In what follows denote $\alpha=\left(\left[\mathbf{U}^{\top}\right]_{1},\left[\mathbf{V}^{\top}\right]_{2},\left[\mathbf{U}^{\top} \otimes \mathbf{V}^{\top}-\mathbf{R}\right]_{1},[\mathbf{R}]_{2}\right)$ where $\mathbf{U} \leftarrow \mathcal{U}_{S}, \mathbf{V} \leftarrow \mathcal{V}_{S}, \mathbf{R} \leftarrow \mathbb{Z}_{q}^{n^{2} \times|S|^{2}}$.

We have

0. $\alpha,\left[(\mathbf{U} \otimes \mathbf{V})^{\top} \boldsymbol{k}-\boldsymbol{r}\right]_{1},[\boldsymbol{r}]_{2}:$ $r \leftarrow \mathbb{Z}_{q}^{n^{2}}, k \leftarrow \mathbb{Z}_{q}^{n^{2}}$

1. $\alpha,\left[(\mathbf{U} \otimes \mathbf{V})^{\top}\left(\boldsymbol{k}_{1} \otimes \boldsymbol{k}_{2}\right)-\boldsymbol{r}\right]_{1},[\boldsymbol{r}]_{2}$ : $r \leftarrow \mathbb{Z}_{q}^{n^{2}}, \quad k_{1}, k_{2} \leftarrow \mathbb{Z}_{q}^{n}$

2. $\alpha,\left[\left(\mathbf{U}^{\top} \boldsymbol{k}_{1}\right) \otimes\left(\mathbf{V}^{\top} \boldsymbol{k}_{2}\right)-\boldsymbol{r}\right]_{1},[\boldsymbol{r}]_{2}$ : $r \leftarrow \mathbb{Z}_{q}^{n^{2}}, \quad k_{1}, k_{2} \leftarrow \mathbb{Z}_{q}^{n}$

3. $\alpha,\left[\boldsymbol{u} \otimes\left(\mathbf{V}^{\top} \boldsymbol{k}_{2}\right)-\boldsymbol{r}\right]_{1},[\boldsymbol{r}]_{2}$ : $u \leftarrow \mathbb{Z}_{q}^{n}, \quad r \leftarrow \mathbb{Z}_{q}^{n^{2}}, \quad \boldsymbol{k}_{2} \leftarrow \mathbb{Z}_{q}^{n}$

4. $\alpha,[r]_{1},\left[\boldsymbol{u} \otimes\left(\mathbf{V}^{\top} \boldsymbol{k}_{2}\right)-\boldsymbol{r}\right]_{2}$ : $u \leftarrow \mathbb{Z}_{q}^{n}, \quad r \leftarrow \mathbb{Z}_{q}^{n^{2}}, \quad k_{2} \leftarrow \mathbb{Z}_{q}^{n}$

5. $\alpha,[r]_{1},[u \otimes v-r]_{2}$ : $u, v \leftarrow \mathbb{Z}_{q}^{n}, \quad r \leftarrow \mathbb{Z}_{q}^{n^{2}}$

6. $\alpha,[s]_{1},[t]_{2}$ : $s, t \leftarrow \mathbb{Z}_{q}^{n^{2}}$

We next show that for all $1 \leq i \leq 5$ the distributions $i-1, i$ are computationally indistinguishable. 
- Case $i=1$. We show that distinguishing these two distributions reduces to the rank problem in $\mathbb{G}_{1}$ introduced in [Vil12], namely, distinguishing $[\mathbf{A}]_{1} \in \mathbb{G}_{1}^{n \times n}$ sampled uniformly over all matrices in $\mathbb{G}_{1}^{n \times n}$ of rank 1 , from $[\mathbf{A}]_{1} \in \mathbb{G}_{1}^{n \times n}$ sampled uniformly over all matrices in $\mathbb{G}_{1}^{n \times n}$ of rank $n$. Now, assume there exists a distinguisher $\mathcal{A}$ for distributions 0 and 1. We construct a distinguisher $\mathcal{B}$ against the rank problem. The distinguisher works as follows: on input $[\mathbf{A}]_{1}$, it samples $\mathbf{U} \leftarrow \mathcal{U}_{S}, \mathbf{V} \leftarrow \mathcal{V}_{S}, \mathbf{R} \leftarrow \mathbb{Z}_{q}^{n^{2} \times|S|^{2}}, r \leftarrow \mathbb{Z}_{q}^{n^{2}}$. It computes $\mathbf{M}=\mathbf{U}^{\top}[\mathbf{A}]_{1} \mathbf{V}$ and vectorizes it; denote the vectorization as $[m]_{1}$. it then executes $\mathcal{A}\left(\left[\mathbf{U}^{\top}\right]_{1},\left[\mathbf{V}^{\top}\right]_{2},\left[\mathbf{U}^{\top} \otimes \mathbf{V}^{\top}-\mathbf{R}\right]_{1},[\mathbf{R}]_{2},[m]_{1}-[r]_{1},[r]_{2}\right)$ and outputs whatever $\mathcal{A}$ outputs. Now, note the vectorization $[m]_{1}$ corresponds to the value $[(\mathbf{U} \otimes \mathbf{V}) m]_{1}$. If $[\mathbf{A}]$ is of rank 1, then we can write $\mathbf{A}=\boldsymbol{k}_{1} \boldsymbol{k}_{2}^{\top}$ and we have $\mathbf{M}=\mathbf{U}^{\top} \boldsymbol{k}_{1} \boldsymbol{k}_{2}^{\top} \mathbf{V}=\mathbf{U}^{\top} \boldsymbol{k}_{1}\left(\mathbf{V}^{\top} \boldsymbol{k}_{2}\right)^{\top}$ and the vectorization corresponds to $\left(\mathbf{U}^{\top} \boldsymbol{k}_{1}\right) \otimes\left(\mathbf{V}^{\top} \boldsymbol{k}_{2}\right)$, namely the case $i=0$. Otherwise, [A] is of rank $n$, and we can write its vectorization as $k$. Then, $m$ correspond to $\left.\left(\mathbf{U}^{\top} \otimes \mathbf{V}^{\top}\right) k\right)$, namely the case $i=1$. As shown in [Vil12], the rank problem reduces to DDH with a security $\operatorname{loss}$ of $\log n$.

- Case $i=2$. Distributions 1,2 are perfectly indistinguishability since the only difference is that the latter is computed as $\left[\left(\mathbf{U}^{\top} \boldsymbol{k}_{1}\right) \otimes\left(\mathbf{V}^{\top} \boldsymbol{k}_{2}\right)-\boldsymbol{r}\right]_{1}$, which equals to $\left[\left(\mathbf{U}^{\top} \otimes \mathbf{V}^{\top}\right)\left(\boldsymbol{k}_{1} \otimes\right.\right.$ $\left.\left.k_{2}\right)-r\right]_{1}$, which is the corresponding value of distribution 1 .

- Case $i=3$. This case reduces to the $\mathcal{U}_{S}^{\top}-\mathrm{MDDH}_{1}$ assumption. The only difference is that in the forth distribution, we replace $\mathbf{U}^{\top} \boldsymbol{k}_{1}$ with a uniform element $\boldsymbol{u}$. It is enough to show that we can compute the rest of the values given $[\mathbf{U}]_{1},[u]_{1}$ where $[u]$ is either $\mathbf{U}^{\top} \boldsymbol{k}_{1}$ or uniform. We can compute the values as

$$
\left[\mathbf{U}^{\top}\right]_{1},\left[\mathbf{V}^{\top}\right]_{2},\left[\mathbf{U}^{\top}\right]_{1} \otimes \mathbf{V}^{\top}-[\mathbf{R}]_{1},[\mathbf{R}]_{2},[\boldsymbol{u}]_{1} \otimes\left(\mathbf{V}^{\top} \boldsymbol{k}_{2}\right)-[\boldsymbol{r}]_{1},[\boldsymbol{r}]_{2}
$$

where we sample $\mathbf{V} \leftarrow \mathcal{V}_{S}, \mathbf{R} \leftarrow \mathbb{Z}_{q}^{n^{2} \times|S|^{2}}, r \leftarrow \mathbb{Z}_{q}^{n^{2}}, k_{2} \leftarrow \mathbb{Z}_{q}^{n}$.

- Case $i=4$. The distributions 4 and 5 are perfectly indistinguishable. It is enough to note that in both, the last two elements are uniformly distributed conditioned on their sum of discrete logarithms being equal to $\boldsymbol{u} \otimes\left(\mathbf{V}^{\top} \boldsymbol{k}_{2}\right)$.

- Case $i=5$. This is the same as the case $i=3$ for the value $[v]_{2}$. This case reduces to the $\mathcal{V}_{S}^{\top}-\mathrm{MDDH}_{2}$ assumption. The only difference is that in the last distribution, we replace $\mathbf{V}^{\top} \boldsymbol{k}_{2}$ with a uniform element $\boldsymbol{v}$. It is enough to show that we can compute the rest of the values given $[\mathbf{V}]_{2},[\boldsymbol{v}]_{2}$ where $\boldsymbol{v}$ is either $\mathbf{V}^{\top} \boldsymbol{k}_{2}$ or uniform. We can compute the values as

$$
\left[\mathbf{U}^{\top}\right]_{1},\left[\mathbf{V}^{\top}\right]_{2},[\mathbf{R}]_{1}, \mathbf{U}^{\top} \otimes\left[\mathbf{V}^{\top}\right]_{2}-[\mathbf{R}]_{2},[\boldsymbol{r}]_{1}, \boldsymbol{u} \otimes[\boldsymbol{v}]_{2}-[\boldsymbol{r}]_{2},
$$

where we sample $\mathbf{U} \leftarrow \mathcal{U}_{S}, \mathbf{R} \leftarrow \mathbb{Z}_{q}^{n^{2} \times|S|^{2}}, r \leftarrow \mathbb{Z}_{q}^{n^{2}}, u \leftarrow \mathbb{Z}_{q}^{n}$.

- Case $i=6$. This again reduces to the rank problem in $\mathcal{G}_{2}$. The only difference in the two distributions is that in distribution 5 the sum of the last two elements, namely $\boldsymbol{u} \otimes \boldsymbol{v}$ is a vectorized matrix of rank 1 , namely $u v^{\top}$, while in distribution 6 is a uniformly distributed matrix of rank $n$ (except w.n.p). Given $[\mathbf{A}]_{2} \in \mathbb{G}_{2}^{n \times n}$ either uniform of rank 1 or uniform of rank $n$ we can compute all the other values efficiently as follows. Let $a$ be the vectorization of $\mathbf{T}$. We compute

$$
\left[\mathbf{U}^{\top}\right]_{1},\left[\mathbf{V}^{\top}\right]_{2},\left[\mathbf{U}^{\top} \otimes \mathbf{V}^{\top}-\mathbf{R}\right]_{1},[\mathbf{R}]_{2},[r]_{1},[a]_{2}-[r]_{2},
$$

where $\mathbf{U} \leftarrow \mathcal{U}_{S}, \mathbf{V} \leftarrow \mathcal{V}_{S}, \mathbf{R} \leftarrow \mathbb{Z}_{q}^{n^{2} \times|S|^{2}}, r \leftarrow \mathbb{Z}_{q}^{n^{2}}$. This implies that distinguishing distributions 5, 6 reduces to the rank problem, which in turn reduces to $\mathrm{DDH}$ in $\mathbb{G}_{2}$. 
The proof of oblivious trapdoor generation essentially follows from the oblivious trapdoor generation and index set hiding of the SSB commitments and is similar to the corresponding proofs for the other constructions.

First we show the corresponding lemma to Lemma 3, that is, we construct an indistinguishable crs given only the commitment keys and the matrices $\mathbf{U}, \mathbf{V}, \mathbf{W}$.

Lemma 4. There exists a modified crs generation algorithm $\mathrm{K}^{\prime}$ that on input $\left(\rho, \theta^{\prime}\right)$, where either $\theta^{\prime}=\left(\mathbf{U}, \mathbf{V}, \mathbf{W},[\mathbf{G} \otimes \mathbf{H}-\mathbf{Z}]_{1},[\mathbf{Z}]_{2}\right)$ or $\theta^{\prime}=\left(\mathbf{G}, \mathbf{H}, \mathbf{F},[\mathbf{U} \otimes \mathbf{V}-\mathbf{R}]_{1},[\mathbf{R}]_{2}\right)$ and outputs $a$ crs such that $(\rho, \mathrm{crs})$ are identically distributed to the honest algorithm.

The lemma follows by inspection an by noting that with the given values we can compute the crs for the sum as explained in Lemma 3. Again, w.l.o.g. we use the same name for the two algorithms, namely $\mathrm{K}$ and differentiate them by their input.

We next show that the construction satisfies oblivious extractability.

Theorem 15. Let $\mathcal{U}, \mathcal{V}, \mathcal{W}$ be witness samplable distributions, and $\mathrm{CS}$ be the algebraic commitment scheme of Fig. 3 for which CS $\otimes$ CS is obliviously extractable. Then Construction QAHad of Fig. 7 is $h_{n s}$-strong oblivious where $h_{n s}=\left([\mathbf{G} \otimes \mathbf{H}-\mathbf{Z}]_{1},[\mathbf{Z}]_{2}\right)$. Furthermore,

1. For every PPT $\mathcal{A}$ against index set hiding of $Q A H a d$, there exist an adversary $\mathcal{B}$ against $h_{n s}$-strong index set hiding property of CS such that $\operatorname{Adv}_{\mathrm{ISH}}^{Q A H a d}(\mathcal{A}) \leq 3 \operatorname{Adv}_{\mathrm{ISH}}^{\mathrm{CS}}(\mathcal{B})$.

2. For every $\mathcal{A}$ against oblivious crs generation of $Q A H a d$, there exist an adversary $\mathcal{B}$ against oblivious crs generation of QASum such that $\operatorname{Adv}_{\mathrm{oblv}}^{Q A H a d}(\mathcal{A}) \leq \operatorname{Adv}_{\mathrm{oblv}}^{Q A S u m}(\mathcal{B})$.

Proof. It is enough to show that index set hiding holds and that we can sample a tuple ( $\rho$, crs) indistinguishable from the one we are given, along with a valid trapdoor. This is the case because the commitment keys are perfectly binding in $S^{\prime}$, which means that the witnesses are unique and do not help the (unbounded) distinguisher who can compute them on its own.

$h_{n s}$-Strong Index Set Hidning. Assume there exist sets $S, S^{\prime}$ of size at most $K$ and an adversary $\mathcal{A}$ which distinguishes $\left(\rho, \operatorname{crs}, h_{n s}(\theta)\right)$ sampled for $S$ from $\left(\rho, \operatorname{crs}, h_{n s}(\theta)\right)$ sampled for $S^{\prime}$ with some probability $\alpha$. We construct adversaries $\mathcal{B}$ distinguishing $c k$ sampled for $S$ from $c k$ sampled for $S$ with probability $\beta$ such that $\alpha \leq 2 \beta$.

$\mathcal{B}$ takes as input some $c k$ sampled either for $S$ or $S^{\prime}$ which is parsed as $[G]_{1}$ and honestly computes the crs following $\mathrm{K}$ of Lemma 4 using the values $[\mathbf{G} \otimes \mathbf{H}-\mathbf{Z}]_{1},[\mathbf{Z}]_{2}$ which are included in $h_{n s}$ except that $[\mathbf{H}]_{2}, \mathbf{H}, \mathbf{T}_{\mathbf{H}},[\mathbf{F}]_{1}, \mathbf{F}, \mathbf{T}_{\mathbf{F}}$ are computed as follows: it samples $b \leftarrow\{0,1\}$ and if $b=0$ it sets

$$
\left([\mathbf{H}]_{2}, \mathbf{H}, \mathbf{T}_{\mathbf{H}}\right) \leftarrow \text { CS.KeyGen }\left(g k_{2}, d, K, S\right), \quad\left([\mathbf{F}]_{1}, \mathbf{F}, \mathbf{T}_{\mathbf{F}}\right) \leftarrow \text { CS.KeyGen }\left(g k_{1}, d, K, S\right)
$$

otherwise it sets

$$
\left([\mathbf{H}]_{2}, \mathbf{H}, \mathbf{T}_{\mathbf{H}}\right) \leftarrow \text { CS.KeyGen }\left(g k_{2}, d, K, S^{\prime}\right), \quad\left([\mathbf{F}]_{1}, \mathbf{F}, \mathbf{T}_{\mathbf{F}}\right) \leftarrow \mathrm{CS} . K e y G e n\left(g k_{1}, d, K, S^{\prime}\right)
$$

If the guess $b$ is correct, by witness samplability of $\mathbf{U}, \mathbf{V}, \mathbf{W}$ the distribution of $\rho$ is not changed, and since the crs is computed as an honest one conditioned on $\rho$, index set hiding follows holds with probability $\frac{\alpha}{2}$. 
Oblivious trapdoor generation: Here, we can simply use the oblivious trapdoor generation of protocol QASum. The conditions of corollary 5 are satisfied since we include the values $[\mathbf{G} \otimes$ $\mathbf{H}-\mathbf{Z}]_{1},[\mathbf{Z}]_{2}$ in $h_{n s}$ and by Thm 6 the commitment key for the sum has oblivious trapdoor generation. It is enough to show that we can compute the crs for the QAHad given a crs for QASum. But this is easy since when given a pair $\left(\rho_{\text {sum }}, \mathrm{crs}_{\text {sum }}\right)$ we execute the oblivious crs algorithm QASum.OblKeyGen $(\rho, \operatorname{crs}, S=(S, S))$ as in Lemma 4.

Corollary 9. If CS is the one from fig. 3, then QAHad from fig. 7 is $h_{n s}$-strong no-signaling where $h_{n s}=\left([\mathbf{G} \otimes \mathbf{H}-\mathbf{Z}]_{1},[\mathbf{Z}]_{2}, \mathbf{U}, \mathbf{V}, \mathbf{W}\right)$.

Proof. The proof follows directly from Theorem 7 and the $h_{n s}$-strong oblivious trapdoor generation of QAHad which is shown in Thm. 15. 


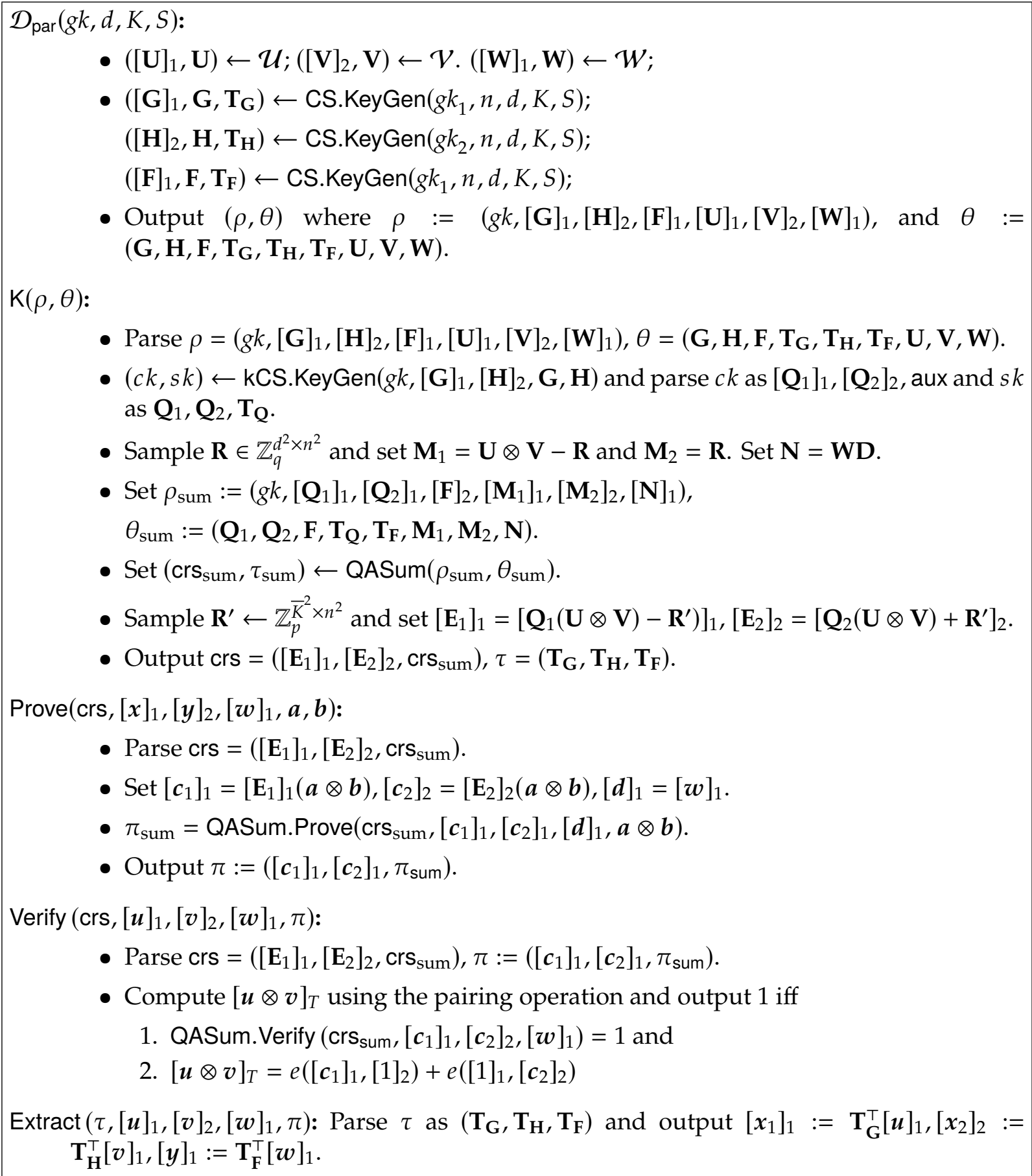

- Parse $\rho=\left(g k,[\mathbf{G}]_{1},[\mathbf{H}]_{2},[\mathbf{F}]_{1},[\mathbf{U}]_{1},[\mathbf{V}]_{2},[\mathbf{W}]_{1}\right), \theta=\left(\mathbf{G}, \mathbf{H}, \mathbf{F}, \mathbf{T}_{\mathbf{G}}, \mathbf{T}_{\mathbf{H}}, \mathbf{T}_{\mathbf{F}}, \mathbf{U}, \mathbf{V}, \mathbf{W}\right)$.

- $(c k, s k) \leftarrow$ kCS.KeyGen $\left(g k,[\mathbf{G}]_{1},[\mathbf{H}]_{2}, \mathbf{G}, \mathbf{H}\right)$ and parse $c k$ as $\left[\mathbf{Q}_{1}\right]_{1},\left[\mathbf{Q}_{2}\right]_{2}$, aux and $s k$ as $\mathbf{Q}_{1}, \mathbf{Q}_{2}, \mathbf{T}_{\mathbf{Q}}$.

- Sample $\mathbf{R} \in \mathbb{Z}_{q}^{d^{2} \times n^{2}}$ and set $\mathbf{M}_{1}=\mathbf{U} \otimes \mathbf{V}-\mathbf{R}$ and $\mathbf{M}_{2}=\mathbf{R}$. Set $\mathbf{N}=\mathbf{W D}$.

- Set $\rho_{\text {sum }}:=\left(g k,\left[\mathbf{Q}_{1}\right]_{1},\left[\mathbf{Q}_{2}\right]_{1},[\mathbf{F}]_{2},\left[\mathbf{M}_{1}\right]_{1},\left[\mathbf{M}_{2}\right]_{2},[\mathbf{N}]_{1}\right)$, $\theta_{\text {sum }}:=\left(\mathbf{Q}_{1}, \mathbf{Q}_{2}, \mathbf{F}, \mathbf{T}_{\mathbf{Q}}, \mathbf{T}_{\mathbf{F}}, \mathbf{M}_{1}, \mathbf{M}_{2}, \mathbf{N}\right)$.

- Set $\left(\operatorname{crs}_{\text {sum }}, \tau_{\text {sum }}\right) \leftarrow \operatorname{QASum}\left(\rho_{\text {sum }}, \theta_{\text {sum }}\right)$.

- Sample $\mathbf{R}^{\prime} \leftarrow \mathbb{Z}_{p}^{\bar{K}^{2} \times n^{2}}$ and set $\left.\left[\mathbf{E}_{1}\right]_{1}=\left[\mathbf{Q}_{1}(\mathbf{U} \otimes \mathbf{V})-\mathbf{R}^{\prime}\right)\right]_{1},\left[\mathbf{E}_{2}\right]_{2}=\left[\mathbf{Q}_{2}(\mathbf{U} \otimes \mathbf{V})+\mathbf{R}^{\prime}\right]_{2}$.

- Output crs $=\left(\left[\mathbf{E}_{1}\right]_{1},\left[\mathbf{E}_{2}\right]_{2}, \mathrm{crs}_{\text {sum }}\right), \tau=\left(\mathbf{T}_{\mathbf{G}}, \mathbf{T}_{\mathbf{H}}, \mathbf{T}_{\mathbf{F}}\right)$.

Prove $\left(\mathrm{crs},[x]_{1},[y]_{2},[w]_{1}, \boldsymbol{a}, \boldsymbol{b}\right)$ :

- Parse crs $=\left(\left[\mathrm{E}_{1}\right]_{1},\left[\mathrm{E}_{2}\right]_{2}, \mathrm{crs}_{\mathrm{sum}}\right)$.

- Set $\left[c_{1}\right]_{1}=\left[\mathrm{E}_{1}\right]_{1}(\boldsymbol{a} \otimes \boldsymbol{b}),\left[\boldsymbol{c}_{2}\right]_{2}=\left[\mathrm{E}_{2}\right]_{2}(\boldsymbol{a} \otimes \boldsymbol{b}),[\boldsymbol{d}]_{1}=[\boldsymbol{w}]_{1}$.

- $\pi_{\text {sum }}=$ QASum.Prove $\left(\mathrm{crs}_{\text {sum }},\left[c_{1}\right]_{1},\left[c_{2}\right]_{1},[d]_{1}, a \otimes b\right)$.

- Output $\pi:=\left(\left[c_{1}\right]_{1},\left[c_{2}\right]_{1}, \pi_{\text {sum }}\right)$.

Verify $\left(\mathrm{crs},[\boldsymbol{u}]_{1},[\boldsymbol{v}]_{2},[\boldsymbol{w}]_{1}, \pi\right)$ :

- Parse crs $=\left(\left[\mathrm{E}_{1}\right]_{1},\left[\mathrm{E}_{2}\right]_{2}, \mathrm{crs}_{\text {sum }}\right), \pi:=\left(\left[c_{1}\right]_{1},\left[c_{2}\right]_{1}, \pi_{\text {sum }}\right)$.

- Compute $[u \otimes v]_{T}$ using the pairing operation and output 1 iff

1. QASum.Verify $\left(\mathrm{crs}_{\text {sum }},\left[c_{1}\right]_{1},\left[c_{2}\right]_{2},[w]_{1}\right)=1$ and

2. $[\boldsymbol{u} \otimes \boldsymbol{v}]_{T}=e\left(\left[\boldsymbol{c}_{1}\right]_{1},[1]_{2}\right)+e\left([1]_{1},\left[\boldsymbol{c}_{2}\right]_{2}\right)$

Extract $\left(\tau,[u]_{1},[\boldsymbol{v}]_{2},[\boldsymbol{w}]_{1}, \pi\right)$ : Parse $\tau$ as $\left(\mathbf{T}_{\mathbf{G}}, \mathbf{T}_{\mathbf{H}}, \mathbf{T}_{\mathbf{F}}\right)$ and output $\left[\boldsymbol{x}_{1}\right]_{1}:=\mathbf{T}_{\mathbf{G}}^{\top}[\boldsymbol{u}]_{1},\left[\boldsymbol{x}_{2}\right]_{2}:=$ $\mathbf{T}_{\mathbf{H}}^{\top}[v]_{1},[y]_{1}:=\mathbf{T}_{\mathbf{F}}^{\top}[w]_{1}$.

Figure 7: Quasi argument QAHad for knowledge transfer of hadammard product. Here $\mathbf{D} \in$ $\mathbb{Z}_{q}^{n \times n^{2}}$ is the matrix such that $\mathrm{D}(\boldsymbol{a} \otimes \boldsymbol{b})=\boldsymbol{a} \circ \boldsymbol{b}$ 


\section{Delegation for Arithmetic Circuit Evaluation}

Formally, we define a delegation scheme as follows.

Definition 10. A triplet of algorithms Del $=$ (Setup, Prove, Verify) is a delegation scheme for circuit evaluation with preprocessing if for any circuit $C: \mathbb{Z}_{p}^{n_{0}} \rightarrow \mathbb{Z}_{p}^{n_{d}}$ :

Completeness: For any $x, y$ such that $y=C(x)$ it holds

$$
\operatorname{Pr}_{g k \leftarrow \mathcal{G}\left(1^{\kappa}\right)}[\operatorname{Verify}(\operatorname{crs}, x, y, \pi)=1 \mid \operatorname{crs} \leftarrow \operatorname{Setup}(g k, C), \pi \leftarrow \operatorname{Prove}(\operatorname{crs}, x, y)] \geq 1-\operatorname{negl}(\kappa),
$$

Soundness: For any adversary $\mathcal{A}$ it holds that

$$
\operatorname{Pr}_{g k \leftarrow \mathcal{G}\left(1^{\kappa}\right)}[\operatorname{Verify}(\operatorname{crs}, x, y, \pi)=1 \text { and } y \neq C(x) \mid \operatorname{crs} \leftarrow \operatorname{Setup}(g k, C),(x, y, \pi) \leftarrow \mathcal{A}(\operatorname{crs})] \leq \operatorname{negl}(\kappa),
$$

Efficiency: The setup algorithm and the prover run in time poly $(|C|, \kappa)$. The size of each proof is $O(\kappa)$ and verification time $n \operatorname{poly}(\kappa)+\operatorname{poly}(\kappa)$.

\subsection{The Scheme}

In the delegation scheme from [GR19] the prover, gives $3 d$ commitments $\left[L_{1}\right]_{1}, \ldots,\left[L_{d}\right]_{1}$, $\left[R_{1}\right]_{2}, \ldots,\left[R_{d}\right]_{2},\left[O_{1}\right]_{1}, \ldots,\left[O_{d}\right]_{1}$ to, respectively, the left, right and output wires of each level of the circuit. Then, it gives a linear and quadratic knowledge transfer arguments to "transfer" knowledge of the opening from the input level, which is known to the verifier, to the next levels. Finally, the verifier checks that the commitment to the output opens to $y$.

We give a "compressed" version of [GR19] where the $3 d$ commitments are shrunken into 3 no-signaling SSB commitments, and the $2 d$ knowledge transfer arguments are shrunk into 2 quasi arguments. From the SSB commitments we can extract $\left[L_{i}\right]_{1}\left[R_{i}\right]_{2}$, $\left[O_{j}\right]_{1}$ for $j=i-1$ or $j=i$. Local knowledge soundness of the quasi arguments imply that knowledge is "transferred" from $\left[O_{i-1}\right]_{1}$ to $\left[L_{i}\right]_{1},\left[R_{i}\right]_{2}$ or from $\left[L_{i}\right]_{1},\left[R_{i}\right]_{2}$ to $\left[O_{i}\right]_{1}$. One important technical problem with this approach is that the linear knowledge transfer argument is between the next level and all previous levels. That is, the knowledge is transferred from commitments to the output in all previous levels $\left[O_{1}\right]_{1}, \ldots,\left[O_{i}\right]_{1}$, to commitments to the left and right wires in the next level $\left[L_{i+1}\right]_{1},\left[R_{i+1}\right]_{2}$. This means the quasi-argument must extract $O(d)$ values and hence is not succinct. We solve this issue by computing $L_{i}, R_{i}, O_{i}$ as commitments also to the respective wires of all previous levels. Consider an arithmetic circuit $C: \mathbb{Z}_{p}^{n_{0}} \rightarrow \mathbb{Z}_{p}^{n_{d}}$. The circuit can be naturally sliced into $d+1$ levels, where level 0 contains the input and level $i$ is formed by a set of $n_{i}$ multiplication gates, the inputs of which depends on a linear transformation of outputs of previous levels. ${ }^{19}$ Let $N_{i}=\sum_{j=0}^{i}$ and $N=N_{d}$. Denote by $\boldsymbol{a}_{i}, \boldsymbol{b}_{i}, \boldsymbol{c}_{i} \in \mathbb{Z}_{p}^{N_{i}}$ the left, right and output wires of level $1, \ldots, i$ respectively. That is $\boldsymbol{a}_{i}=\left(\begin{array}{c}\boldsymbol{a}_{i-1} \\ \mathbf{D}_{i} c_{i-1}\end{array}\right)$ and $\boldsymbol{b}_{i}=\left(\begin{array}{c}\boldsymbol{b}_{i-1} \\ \mathbf{E}_{i} c_{i-1}\end{array}\right)$, where $\mathbf{D}_{i}, \mathbf{E}_{i} \in \mathbb{Z}_{p}^{n_{i} \times N_{i-1}}$ are defined by the circuit's linear gates, $\boldsymbol{a}_{0}, \boldsymbol{b}_{0}$ are of size 0 and $\boldsymbol{c}_{0}=\boldsymbol{x}$ is the input. Let $\mathbf{D} \in \mathbb{Z}_{p}^{N-n_{0} \times N}$ (resp. E) be the matrix such that the $i$-th row of $\mathbf{D}$ is $\left(\mathbf{D}_{i} \mid \mathbf{0}_{n_{i} \times N-N_{i-1}}\right)$. Note that matrices D, E are lower triangular. For the outputs we have $\boldsymbol{c}_{i}=\boldsymbol{a}_{i} \circ \boldsymbol{b}_{i}$.

Denote $\boldsymbol{a}=\boldsymbol{a}_{d}, \boldsymbol{b}=\boldsymbol{b}_{d}$ and $\boldsymbol{c}=\boldsymbol{c}_{d-1}$. The evaluation of the circuit is correct if $\left(\begin{array}{l}a \\ b\end{array}\right)=\left(\begin{array}{l}\mathrm{D} \\ \mathrm{E}\end{array}\right) \boldsymbol{c}$ and $\boldsymbol{c}=\boldsymbol{a} \circ \boldsymbol{b}$. Next, consider Pedersen commitment keys $\mathbf{U}_{i}^{*} \leftarrow \mathbb{Z}_{p}^{1 \times n_{i}}, \mathbf{V}_{i}^{*} \leftarrow \mathbb{Z}_{p}^{1 \times n_{i}}$ and $\mathbf{W}_{i}^{*} \leftarrow \mathbb{Z}_{p}^{1 \times n_{i}}$ and define $\mathbf{U}_{i}=\left(\mathbf{U}_{1}^{*}, \ldots, \mathbf{U}_{i}^{*}\right), \mathbf{V}_{i}=\left(\mathbf{V}_{1}^{*}, \ldots, \mathbf{V}_{i}^{*}\right)$, for $i \in[d], \mathbf{W}_{i}=\left(\mathbf{W}_{1}^{*}, \ldots, \mathbf{W}_{i}^{*}\right)$,

${ }^{19}$ We consider w.l.o.g. only linear transformations since if we can handle affine ones by including a wire with the value 1 in the input. 
for $i \in[d-1]$. Consider commitments (represented in $\mathbb{Z}_{p}$ ) to left, right and output wires as $O_{i}=\mathbf{W}_{i} \boldsymbol{c}_{i}, \boldsymbol{O}=\mathbf{W} \boldsymbol{c}, L_{i}=\mathbf{U}_{i} \boldsymbol{a}_{i}=\mathbf{U} \boldsymbol{a}, R_{i}=\mathbf{V}_{i} \boldsymbol{b}_{i}, \mathbf{R}=\mathbf{V} \boldsymbol{b}$, where

$$
\mathbf{U}=\left(\begin{array}{ccc}
\mathbf{U}_{1}^{*} & & \mathbf{0} \\
\vdots & \ddots & \\
\mathbf{U}_{1}^{*} & \ldots & \mathbf{U}_{d}^{*}
\end{array}\right), \mathbf{V}=\left(\begin{array}{ccc}
\mathbf{V}_{1}^{*} & & \mathbf{0} \\
\vdots & \ddots & \\
\mathbf{V}_{1}^{*} & \ldots & \mathbf{V}_{d}^{*}
\end{array}\right), \mathbf{W}=\left(\begin{array}{ccc}
\mathbf{W}_{1}^{*} & & \mathbf{0} \\
\vdots & \ddots & \\
\mathbf{W}_{1}^{*} & \ldots & \mathbf{W}_{d-1}^{*}
\end{array}\right),
$$

$\boldsymbol{O}=\left(O_{1}, \ldots, O_{d-1}\right)^{\top}, \boldsymbol{L}=\left(L_{1}, \ldots, L_{d}\right)^{\top}, \boldsymbol{R}=\left(R_{1}, \ldots, R_{d}\right)^{\top}$.

We additionally pick $\mathbf{G}, \mathbf{H}, \mathbf{F}$ for computing SSB commitments to vectors of size $d$ and publish $[\mathbf{G U}]_{1},[\mathbf{H V}]_{2},[\mathbf{F W}]_{2}$. The prover computes $[\hat{\mathbf{L}}]_{1}=[\mathbf{G U}]_{1} \boldsymbol{a},[\hat{\mathbf{R}}]_{2}=[\mathbf{H V}]_{2} \boldsymbol{b},[\hat{\mathbf{O}}]_{1}=$ $[\mathbf{F W}]_{1} c$ and gives a quasi-argument of linear knowledge transfer from $x,[O]_{1}, y$ to $[L]_{1},[R]_{2}$ with the following structure

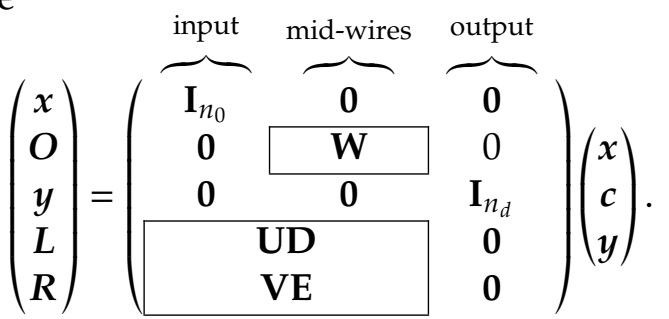

That is, we can extract $\left[L_{i}\right]_{1},\left[R_{i}\right]_{2},\left[O_{i-1}\right]_{1}$ and, if we are additionally given $\boldsymbol{c}_{i-1}$ such that $O_{i-1}=$ $\mathbf{W}_{i-1} \boldsymbol{c}_{i-1}$, then $L_{i}=\mathbf{U}_{i} \mathbf{D}_{i} \boldsymbol{c}_{i}, R_{i}=\mathbf{V}_{i} \mathbf{E}_{i} \boldsymbol{c}_{i}$. We also use a quasi-argument of knowledge transfer of the hadamard product from $[L]_{1},[R]_{2}$ to $[O]_{1}$. In this case we extract $\left[L_{i}\right]_{1},\left[R_{i}\right]_{2},\left[O_{i}\right]_{1}$ and, if we are additionally given $\boldsymbol{a}_{i}, \boldsymbol{b}_{i}$ such that $L_{i}=\mathbf{U}_{i} \boldsymbol{a}_{i}$ and $R_{i}=\mathbf{V}_{i} \boldsymbol{b}_{i}$, then $O_{i}=\mathbf{W}_{i}\left(\boldsymbol{a}_{i} \circ \boldsymbol{b}_{i}\right)$.

We need to make one last change that will allow us to take into account the input $x$ and the claimed output $y$. Essentially, we make the first and last commitment key (trivially) perfectly binding by using as a commitment key the identity matrix. The security properties still hold in a trivial way (the $\mathbf{I}_{n_{0}}-\mathrm{MDDH}$ assumption is perfectly secure). We change accordingly the SSB commitment key, that is we set $\mathbf{F}^{\prime}=\left(\begin{array}{ccc}\mathbf{I}_{n_{0}} & 0 & 0 \\ 0 & \mathbf{F} & 0 \\ 0 & 0 & \mathbf{I}_{n_{d}}\end{array}\right)$. Note that the extraction trapdoor remains the same, but the extractor can trivially extract the values corresponding to $x, y$ regardless of $\mathbf{F}^{\prime}$ distribution. In other words, our commitment key is always perfectly binding in the first $n_{0}$ and $n_{d}$ coordinates. We denote with $\mathbf{W}^{\prime}$ the modified matrix where we change the first and last rows with $\left(\mathbf{I}_{n_{0}} \mid \mathbf{0}\right)$ and $\left(\mathbf{0} \mid \mathbf{I}_{n_{d}}\right)$ respectively. Therefore, if $\boldsymbol{O}=\mathbf{W}^{\prime} \boldsymbol{c}$, we get that $O_{0}=\boldsymbol{x}$ and $O_{d}=y$.

\subsection{Proof of Security}

Theorem 16. Let $\mathcal{A}$ be an adversary against Adaptive Soundness of the delegation scheme of Fig. 8, that outputs an input/output pair $\boldsymbol{x}, \boldsymbol{y}^{*}$ and a valid proof $\pi:=\left([\hat{\boldsymbol{L}}]_{1},[\hat{\mathbf{R}}]_{2},[\hat{\boldsymbol{O}}]_{1}, \pi_{\mathrm{had}}, \pi_{\text {blin }}\right)$ but $\boldsymbol{y}^{*} \neq C(\boldsymbol{x})$. Then there exists a distinguisher $\mathcal{D}_{\text {blin }}, \mathcal{D}_{\text {had }}$ and adversaries $\mathcal{B}_{\text {blin }}, \mathcal{B}_{\text {had }}$ against the no-signaling property of QABlin and QAHad, respectively, and adversaries $\mathcal{A}_{\text {blin, }} \mathcal{A}_{\text {had }}$ against local knowledge soundness of QABlin and local knowledge soundness QAHad, respectively, such that

$$
\begin{aligned}
\operatorname{Adv}_{\text {Del }}(\mathcal{A}) & \leq 2(d+1)\left(\operatorname{Adv}_{\text {NS }}^{\text {QAHad }}\left(\mathcal{D}_{\text {had }}, \mathcal{B}_{\text {had }}\right)+\operatorname{Adv}_{\text {NS }}^{\text {QAHad }}\left(\mathcal{D}_{\text {had }}, \mathcal{B}_{\text {had }}\right)\right) \\
& +(d+1)\left(\operatorname{Adv}_{\text {snd }}^{\text {QAHad }}\left(\mathcal{A}_{\text {had }}\right)+\operatorname{Adv}_{\text {snd }}^{\text {QABLin }}\left(\mathcal{A}_{\text {blin }}\right)\right)
\end{aligned}
$$

Proof. Let $\mathrm{Game}_{0}$ be the soundness game: 


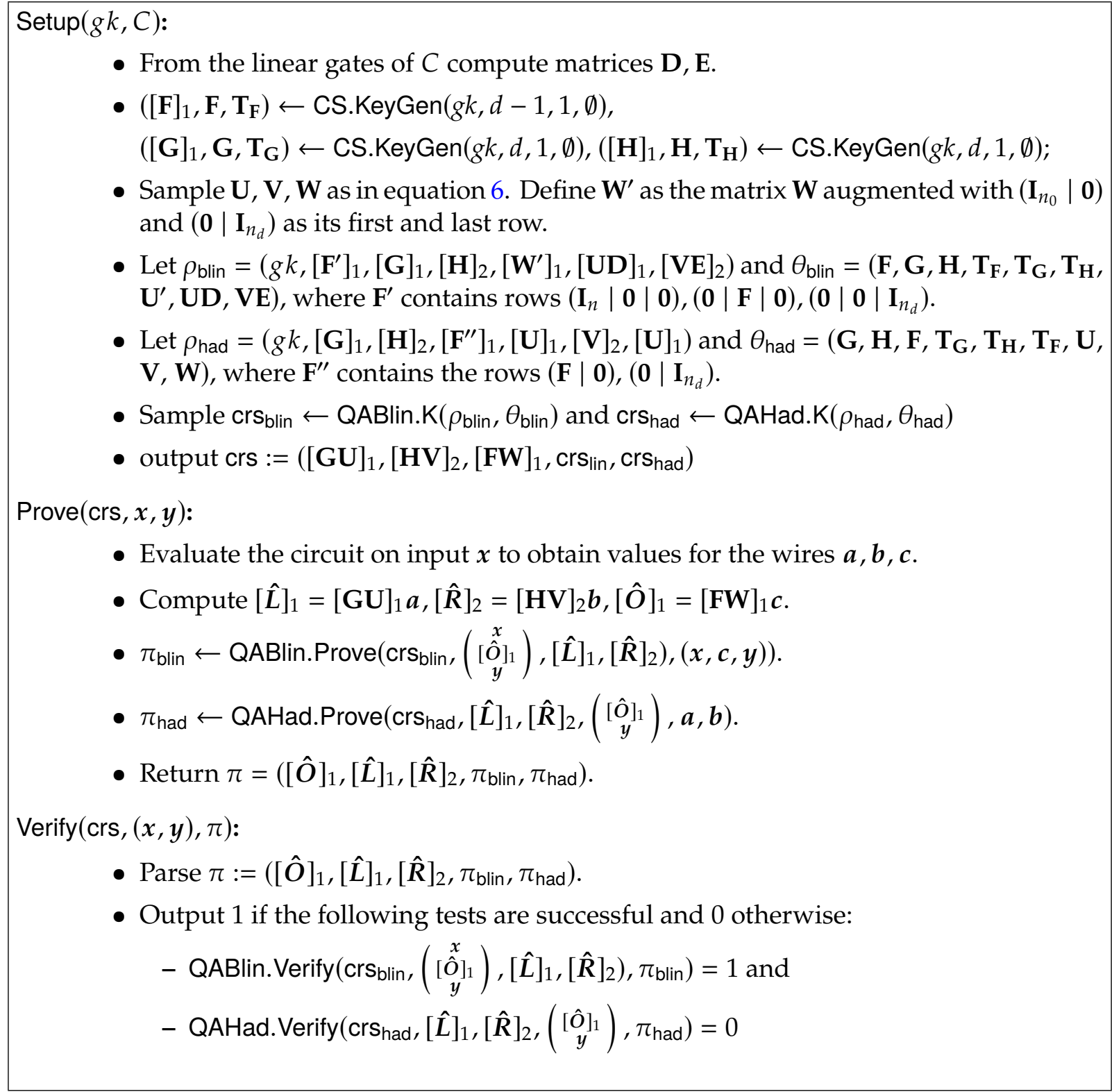

Figure 8: Delegation scheme for an arithmetic circuit.

Game $_{0}$ : This is the soundness game. The output of $\mathrm{Game}_{0}$ is 1 iff on input crs $\leftarrow \operatorname{Setup}(g k, C)$, the adversary outputs $x, y, \pi \leftarrow \mathcal{A}(\mathrm{crs})$ such that $C(x) \neq y$ and the proof verifies, namely $\operatorname{Verify}(\operatorname{crs}, x, y, \pi)=1$.

In what follows we use the fact that the commitment keys corresponding to $[O]_{0}$ and $[O]_{d}$ are the identity matrices. Therefore are trivially extractable; thus the bilateral knowledge argument is sound since it satisfies the soundness conditions (MDDH is trivially hard for the identity matrix). This is used in the same way as [GR19].

For $i \in[d], j \in[0, d]$ and $S_{1}, S_{2}$ sets of sizes at most 1 , consider the following games:

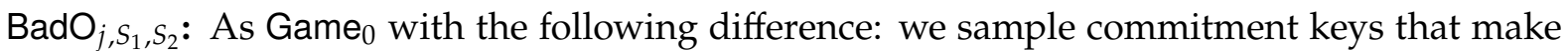

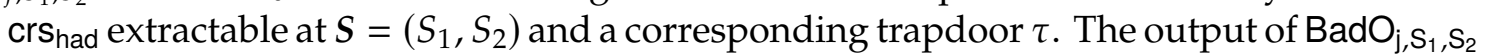
is 1 iff either $S_{2} \neq\{j\}$ or $\left[O_{j}\right]_{1} \neq\left[\mathbf{W}_{j}^{*}\right]_{1} c_{j}$, where $\boldsymbol{c}_{j}$ is computed by honestly executing $C(\boldsymbol{x})$ and $\left[O_{j}\right]_{1} \leftarrow$ QAHad.Extract $\left(\tau,[\hat{L}]_{1},[\hat{\boldsymbol{R}}]_{2},[\hat{O}]_{1}, \pi_{\text {had }}\right)$ is extracted from the adversary's 
proof $\pi=\left([\hat{O}]_{1},[\hat{L}]_{1},[\hat{R}]_{2}, \pi_{\text {blin }}, \pi_{\text {had }}\right)$.

$\operatorname{BadLR}_{i, S_{1}, S_{2}}$ : As Game $_{0}$ with the difference: we sample commitment keys that make $\mathrm{crs}_{\text {lin }}$ extractable at $S=\left(S_{1}, S_{2}\right)$ and a corresponding trapdoor $\tau$. The output of BadLR $R_{i, S_{1}, S_{2}}$ is 1 iff either $S_{1} \neq\{i\}$ or $\left[L_{i}\right]_{1} \neq\left[\mathbf{U}_{i}^{*}\right]_{1} \boldsymbol{a}_{i}$ or $\left[R_{i}\right]_{1} \neq\left[\mathbf{V}_{i}^{*}\right]_{1} \boldsymbol{b}_{i}$, where $\boldsymbol{a}_{i}, \boldsymbol{b}_{i}$ are computed by honestly executing $C(x)$ and $\left(\left[L_{i}\right]_{1},\left[R_{i}\right]_{1}\right) \leftarrow \operatorname{QAHad}$.Extract $\left(\tau,[\hat{\boldsymbol{L}}]_{1},[\hat{\boldsymbol{R}}]_{2},[\hat{\boldsymbol{O}}]_{1}, \pi_{\text {blin }}\right)$ is extracted from the adversary's proof $\pi=\left([\hat{O}]_{1},[\hat{L}]_{1},[\hat{R}]_{2}, \pi_{\text {blin }}, \pi_{\text {had }}\right)$.

Now for any game, let $E$ be the event where the output $(x, y, \pi) \leftarrow \mathcal{A}$ (crs) satisfies $\operatorname{Verify}(\mathrm{crs}, \boldsymbol{x}, \boldsymbol{y}, \pi)=1$. We define $O_{d}=\boldsymbol{y}$ and $\mathbf{W}_{d}=\left(\mathbf{0}_{n_{d} \times N-n_{d}} \mid \mathbf{I}_{n_{d}}\right)$ so that $\mathrm{Game}_{0}=\mathrm{BadO}_{d, \emptyset,\{d\}} \wedge$ $E$. We also define $\mathrm{BadO}_{i}=\mathrm{BadO}_{i, \emptyset,\{i\}}$.

Let $\mathcal{A}$ be an adversary against adaptive soundness of the delegation scheme and for each $i$ define $\operatorname{Pr}\left[\operatorname{BadO}_{i}^{\mathcal{A}}(g k)=1 \mid E\right]=p_{i}$. We claim that

$$
\begin{aligned}
\operatorname{Pr}\left[\operatorname{BadO}_{i+1}^{\mathcal{A}}(g k)=1 \mid E\right] & \leq p_{i}+\operatorname{Adv}_{\text {snd }}^{\text {QABlin }}\left(\mathcal{A}_{\text {blin }}\right)+\operatorname{Adv}_{\text {snd }}^{\mathrm{QAHad}}\left(\mathcal{A}_{\text {had }}\right) \\
& +2 \operatorname{Adv}_{\mathrm{ns}}^{\mathrm{QABlin}}\left(\mathcal{D}_{\text {blin }}, \mathcal{B}_{\text {blin }}\right)+2 \operatorname{Adv}_{\mathrm{ns}}^{\mathrm{QAHad}}\left(\mathcal{D}_{\text {had }}, \mathcal{B}_{\text {had }}\right)
\end{aligned}
$$

The proof of the claim is by induction over $i$. In the inductive case we show that

$$
\begin{aligned}
\operatorname{Pr}\left[\operatorname{BadO}_{i}^{\mathcal{A}}=1 \mid E\right] & \approx \operatorname{Pr}\left[\operatorname{BadO}_{i,(\{i+1\},\{i\})}^{\mathcal{A}}=1 \mid E\right] \approx \operatorname{Pr}\left[\operatorname{BadLR}_{i,(\{i+1\},\{i\})}^{\mathcal{A}}=1 \mid E\right] \\
& \approx \operatorname{Pr}\left[\operatorname{BadLR}_{i,(\{i+1\},\{i+1\})}^{\mathcal{A}}=1 \mid E\right]
\end{aligned}
$$

and $\operatorname{Pr}\left[\operatorname{BadLR}_{i,(\{i+1\},\{i+1\})}^{\mathcal{A}}=1 \mid E\right] \approx \operatorname{Pr}\left[\operatorname{BadO}_{i+1,(\{i+1\},\{i+1\})}^{\mathcal{A}}=1 \mid E\right] \approx \operatorname{Pr}\left[\operatorname{BadO}_{i+1}^{\mathcal{A}}=1 \mid E\right]$

where $p_{1} \approx p_{2}$ is defined as $\left|p_{1}-p_{2}\right| \leq \operatorname{negl}(\kappa)$. Now we show that each $\approx$ is indeed negligible. Note that $\rho_{\text {had }}$ can be computed from $\rho_{\text {blin }}$ and vice-versa.

$\mathrm{BadO}_{i}, \mathrm{BadO}_{i,(\{i+1\},\{i\})}$ : Consider the sets $S_{1}=(\emptyset,\{i\})$ and $S_{2}=(\{i+1\},\{i\})$. We show that the output of the games relative to $\mathcal{A}$ are computationally indistinguishable by reducing to the no-signaling property of QABlin.

We construct adversaries $\mathcal{D}_{\text {blin }}, \mathcal{B}_{\text {blin }}$ against no-signaling extraction of QABlin. By Corollary 5 , the no-signaling property holds even when $\mathcal{B}_{\text {blin }}$ is given $\rho_{\text {blin }}, \mathrm{crs}$ blin and additionally $h_{\mathrm{ns}}\left(\theta_{\text {blin }}\right)=\left(\mathbf{U}, \mathbf{V}, \mathbf{W},[\mathbf{G} \otimes \mathbf{H}+\mathbf{Z}]_{1},[-\mathbf{Z}]_{2}\right)$. Using this additional help, $\mathcal{B}_{\text {blin }}$ computes $\mathrm{crs}_{\text {had }} \leftarrow$ QAHad.K $\left(\rho_{\text {had }}, \theta^{\prime}=h_{\text {ns }}\left(\theta_{\text {blin }}\right)\right)$ as in Lemma 4 . It then runs $\mathcal{A}($ crs $)$ until it outputs $\left(\boldsymbol{x}, \boldsymbol{y}^{*},[\hat{\boldsymbol{O}}]_{1},[\hat{\boldsymbol{L}}]_{1},[\hat{\boldsymbol{R}}]_{2}, \pi_{\text {blin }}, \pi_{\text {had }}\right)$, and then $\mathcal{B}_{\text {blin }}$ outputs $\left(\left[\begin{array}{c}x \\ \hat{O} \\ y^{*}\end{array}\right]_{1},[\hat{\boldsymbol{L}}]_{1},[\hat{\boldsymbol{R}}]_{2}\right)$ and $\pi_{\text {blin. }}$ It gets the extracted value for the intersection of the two sets $(\emptyset,\{i\})$, namely $\left[O_{i}\right]_{1}$. If all conditions of $\mathrm{BadO}_{i}$ and $E$ hold (Verify $\left(\mathrm{crs}, x^{*}, y^{*}, \pi\right)=1$ and $\left.\left[O_{i}\right] \neq \mathbf{W}_{i}^{*} c_{i}\right) \mathcal{D}_{\text {blin }}$ outputs 1 and otherwise 0 .

Then we can bound $\operatorname{Pr}\left[\operatorname{BadO}_{i,(\{i+1\},\{i\})}^{\mathcal{A}}=1 \mid E\right] \leq p_{i}+\operatorname{Adv}_{\mathrm{NS}}^{\mathrm{QABlin}}\left(\mathcal{D}_{\text {blin }}, \mathcal{B}_{\text {blin }}\right)=p_{i, 1}$.

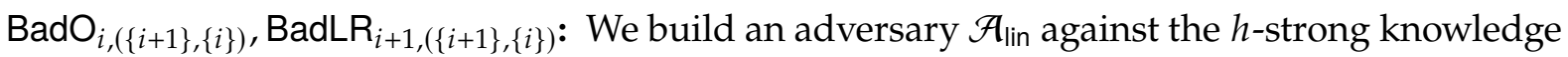
soundness of QABlin. On input crs blin and $h_{\mathrm{ls}}\left(\theta_{\text {blin }}\right)=(\mathbf{G}, \mathbf{H}, \mathbf{F}, \mathbf{U}, \mathbf{V})$ computes crs had $\leftarrow$ QAHad.K $\left(\rho_{\text {had }}, h_{\mathrm{ls}}\left(\theta_{\text {blin }}\right)\right)$, as in Lemma 4 . Then runs $\mathcal{A}(\mathrm{crs})$ until it outputs $\boldsymbol{x}, \boldsymbol{y}^{*}, \pi$ and then $\mathcal{A}_{\text {blin }}$ outputs $\left(\left[\begin{array}{l}x \\ \hat{O} \\ y^{*}\end{array}\right]_{1},[L]_{1},[R]_{2}\right)$ and $\pi_{\text {blin. }}$. Now by definition, conditioned on $E$, if the events $\neg \operatorname{BadO}_{i+1,(\{i+1\},\{i\})}^{\mathcal{A}}$ and $\operatorname{BadLR}_{i+1,(\{i\},\{i\})}^{\mathcal{A}}$ happen, it holds that (1) $\pi_{\text {blin }}$ verifies, (2) $\left[O_{i}\right]_{1}=\mathbf{W}_{i} \boldsymbol{c}_{i}$ and (3) $\left[L_{i+1}\right]_{1} \neq \mathbf{U}_{i+1} \boldsymbol{a}_{i+1}$ or $\left[R_{i+1}\right]_{1} \neq \mathbf{V}_{i+1} \boldsymbol{b}_{i+1}$. Then we can bound

$$
\begin{aligned}
\operatorname{Pr}\left[\operatorname{BadLR}_{i+1,(\{i+1\},\{i\})}^{\mathcal{A}}=1 \mid E\right] & \leq \operatorname{Pr}\left[\operatorname{BadLR}_{i+1,(\{i+1\},\{i\})}^{\mathcal{A}}=1 \wedge \operatorname{BadO}_{i,(\{i+1\},\{i\})}^{\mathcal{A}}=1 \mid E\right] \\
& +\operatorname{Pr}\left[\operatorname{BadLR}_{i+1,(\{i+1\},\{i\})}^{\mathcal{A}}=1 \wedge \neg \operatorname{BadO}_{i,(\{i+1\},\{i\})}^{\mathcal{A}}=1 \mid E\right] \\
& \leq p_{i, 1}+\operatorname{Adv}_{\text {snd }}^{\operatorname{QABLin}}\left(\mathcal{A}_{\mathrm{blin}}\right)=p_{i, 2}
\end{aligned}
$$


$\operatorname{BadLR}_{i+1,(\{i+1\},\{i\})}, \mathrm{BadLR}_{i+1,(\{i+1\},\{i+1\})}$ : Similarly as the case $\mathrm{BadO}_{i}, \mathrm{BadO}_{i,(\{i+1\},\{i\})}$, but we need to transition between sets $(\{i\},\{i+1\}) \rightarrow(\emptyset,\{i+1\}) \rightarrow(\{i+1\},\{i+1\})$. We use twice the no-signaling property of QAHad and exploit the fact that we can build crsblin using $h_{n s}\left(\theta_{\text {had }}\right)$. Therefore, $\operatorname{Pr}\left[\operatorname{BadLR}_{i,(\{i+1\},\{i+1\})}=1 \mid E\right] \leq p_{i, 2}+2 \operatorname{Adv}_{\mathrm{NS}}^{\mathrm{QAHad}}\left(\mathcal{D}_{\text {had }}, \mathcal{B}_{\text {had }}\right)=p_{i, 3}$.

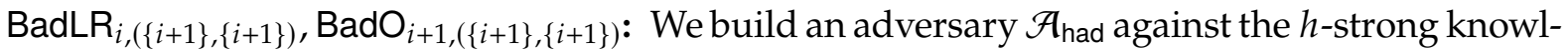
edge soundness of QAHad. On input crs had and $h_{\mathrm{ls}}\left(\theta_{\text {had }}\right)=(\mathbf{G}, \mathbf{H}, \mathbf{F}, \mathbf{W})$ computes $\mathrm{crs}_{\text {blin }} \leftarrow \operatorname{QABlin} . \mathrm{K}\left(\rho_{\text {blin }}, h_{\mathrm{ls}}\left(\theta_{\text {had }}\right)\right)$, as in Lemma 2 . Then runs $\mathcal{A}(\mathrm{crs})$ until it outputs $x, y^{*}, \pi$ and then $\mathcal{A}_{\text {blin }}$ outputs $\left([\mathbf{L}]_{1},[\mathbf{R}]_{2},\left[\begin{array}{c}\hat{O} \\ y^{*}\end{array}\right]_{1}\right)$ and $\pi_{\text {had }}$. Now by definition, conditioned on $E$, if the events $\neg \operatorname{BadLR}_{i+1,(\{i+1\},\{i+1\})}^{\mathcal{A}}$ and $\operatorname{BadO}_{i+1,(\{i+1\},\{i+1\})}^{\mathcal{A}}$ happen, it holds that (1) $\pi_{\text {had }}$ verifies, (2) $\left[L_{i+1}\right]_{1}=\mathbf{U}_{i+1} \boldsymbol{a}_{i+1}$ and $\left[R_{i+1}\right]_{1}=\mathbf{V}_{i+1} \boldsymbol{b}_{i+1}$ and (3) $\left[O_{i+1}\right]_{1} \neq \mathbf{W}_{i+1} \boldsymbol{c}_{i+1}$. Then we can bound

$$
\begin{aligned}
\operatorname{Pr}\left[\operatorname{BadO}_{i+1,(\{i+1\},\{i+1\})}^{\mathcal{A}}=1 \mid E\right] & \leq \operatorname{Pr}\left[\operatorname{BadO}_{i+1,(\{i+1\},\{i+1\})}^{\mathcal{A}}=1 \wedge \operatorname{BadLR}_{i+1,(\{i+1\},\{i+1\})}^{\mathcal{A}}=1 \mid E\right] \\
& +\operatorname{Pr}\left[\operatorname{BadO}_{i+1,(\{i+1\},\{i+1\})}^{\mathcal{A}}=1 \wedge \neg \operatorname{BadLR}_{i+1,(\{i+1\},\{i+1\})}^{\mathcal{A}}=1 \mid E\right] \\
& \leq p_{i, 3}+\operatorname{Adv}_{\text {snd }}^{\operatorname{QAHad}}\left(\mathcal{A}_{\text {had }}\right)=p_{i, 4}
\end{aligned}
$$

$\mathrm{BadO}_{i+1,(\{i+1\},\{i+1\})}, \mathrm{BadO}_{i+1}$ : Similarly as the case $\mathrm{BadO}_{i}, \mathrm{BadO}_{i,\{(i+1\},\{i\})}$ we can bound

$$
\operatorname{Pr}\left[\operatorname{BadO}{ }_{i+1}^{\mathcal{A}}=1 \mid E\right] \leq p_{i, 4}+\operatorname{Adv}_{\mathrm{NS}}^{\mathrm{QABlin}}\left(\mathcal{D}_{\text {blin }}, \mathcal{B}_{\text {blin }}\right)
$$

and we conclude the claim.

Now, $\operatorname{Pr}\left[\operatorname{BadO}_{i+1}^{\mathcal{A}}(g k)=1 \mid E\right]=\operatorname{Pr}\left[\operatorname{BadO}_{i}^{\mathcal{A}}(g k)=1\right]+p_{i}$. We have that

$$
\operatorname{Pr}\left[\operatorname{BadO}_{d}^{\mathcal{A}}(g k)=1 \mid E\right]=\operatorname{Pr}\left[\operatorname{Game}_{0}^{\mathcal{A}}(g k)=1\right]=\sum_{i=0}^{d} p_{i}
$$

which concludes the proof.

Efficiency. The size of the crs is $\left(6 N^{2}+6 N+24\right) \mathbb{G}_{1}$ elements and $\left(6 N^{2}+4 N+36\right) \mathbb{G}_{2}$ elements and computing it is dominated by the same number of group exponentiations in $\mathbb{G}_{1}, \mathbb{G}_{2}$ respectively; the prover is dominated by $6 N^{2}+6 N$ exponentiations in $\mathbb{G}_{1}$ and $6 N^{2}+2 N$ exponentiations in $\mathbb{G}_{2}$ and produces a proof of size $12 \mathbb{G}_{1}+10 \mathbb{G}_{2}$ group elements; verifying a proof requires 36 pairing operations. The size of the proof can be reduced to $10 \mathbb{G}_{1}+8 \mathbb{G}_{2}$ combining the linear argument with the one used by the hadamard quasi argument.

\section{Applications}

In this section we show how to use our delegation scheme to (1) get a NIZK argument for NP in the preprocessing model where the size of the proof is linear in the size of the NP witness and independent of the computation size, in spite of most NIZK constructions under standard assumptions; (2) a zk-SNARK with quantitatively weaker assumptions and (3) compact NIZK for NP with proof size proportional to the witness.

We will use Groth-Sahai proofs [GS08] and, for completeness, we give a high level overview. 
Groth-Sahai Proofs The Groth Sahai (GS) proof system is a non-interactive witness indistinguishable proof system (and in some cases also zero-knowledge) for the language of quadratic equations over a bilinear group. The admissible equation types must be in the following form:

$$
\sum_{j=1}^{m_{y}} f\left(\alpha_{j}, \mathrm{y}_{j}\right)+\sum_{i=1}^{m_{x}} f\left(\mathrm{x}_{i}, \beta_{i}\right)+\sum_{i=1}^{m_{x}} \sum_{j=1}^{m_{y}} f\left(\mathrm{x}_{i}, \gamma_{i, j} \mathrm{y}_{j}\right)=t
$$

where $\boldsymbol{\alpha} \in \mathbb{M}_{1}^{m_{y}}, \boldsymbol{\beta} \in \mathbb{M}_{2}^{m_{x}}, \boldsymbol{\Gamma}=\left(\gamma_{i, j}\right) \in \mathbb{Z}_{q}^{m_{x} \times m_{y}}, t \in \mathbb{M}_{T}$, and $\mathbb{M}_{1}, \mathbb{M}_{2}, \mathbb{M}_{T} \in\left\{\mathbb{Z}_{q}, \mathbb{G}_{1}, \mathbb{G}_{2}, \mathbb{G}_{T}\right\}$ are equipped with some bilinear map $f: \mathbb{M}_{1} \times \mathbb{M}_{2} \rightarrow \mathbb{M}_{T}$. The proof system is also zero-knowledge whenever $\mathbb{M}_{1} \neq \mathbb{G}_{1}$ or $\mathbb{M}_{2} \neq \mathbb{G}_{2}$ or $t=0$ [EG14]. We will use only equations for which $t=0$.

The GS proof system is a commit-and-prove proof system. That is, the prover first commits to solutions of equation 8 using Groth-Sahai commitments ${ }^{20}$, and then computes a proof that the committed values satisfies equation 8 . We denote an instance of the Groth-Sahai proof system by GS $=\left(\operatorname{Setup}_{\mathrm{pb}}, \operatorname{Setup}_{\mathrm{ph}}, \mathrm{P}, \mathrm{V}\right)$.

GS proofs are perfectly sound when the CRS is sampled from the perfectly binding distribution, i.e crs $_{\mathrm{GS}} \leftarrow$ GS.Setup $\mathrm{pb}(g k)$. This means that any $\pi$ such that GS.V( $\operatorname{crs}_{\mathrm{GS}}$, equation $\left.8, \pi\right)=$ 1 contains commitments from which one can extract solutions to equation 8 with probability 1. Proofs are perfectly witness-indistinguishable when sampled from the perfectly hiding distribution, i.e. crs $_{\mathrm{GS}} \leftarrow \mathrm{GS}$. $\operatorname{Setup}_{\mathrm{ph}}(g k)$. That is, for any two solution to equation 8 the proofs follow exactly the same distribution, Computational indistinguishability of GS.Setup and GS.Setup $\mathrm{ph}_{\mathrm{implies}}$ that either the proof system is perfectly sound and computationally witness indistinguishable or computationally sound and perfect witness-indistinguishable.

\subsection{NIZK arguments for NP.}

Let $\mathrm{CS}_{E}$ an be algebraic commitment scheme -namely compatible with the Groth-Sahai proof system [GS08]- which is hiding and extractable. Also note that we can express the verification algorithm Del.Verify as a set of pairing product equation. The idea to construct a NIZK is the following: let $C$ be an arithmetic circuit that takes public input $x$ and secret input $w$ the secret input, and let $\mathrm{crs}_{\text {Del }}$ be a crs for the delegation of computation of $C$. The prover commits to $w$ and the group elements defining the proof of the delegation using the extractable commitment and gives a Groth-Sahai proof that the set of verification equations are satisfied w.r.t. the opening of the commitment. Now, if $\mathrm{CS}_{E}$ is extractable, we can extract the witness $w$, and if the circuit is not satisfied w.r.t. $x, w$ we can break adaptive soundness of delegation scheme Del. We present the scheme.

\footnotetext{
${ }^{20}$ For elements of $\mathbb{Z}_{p}$, a Groth-Sahai commitment is just an SSB commitment wiht locality parameter 1.
} 


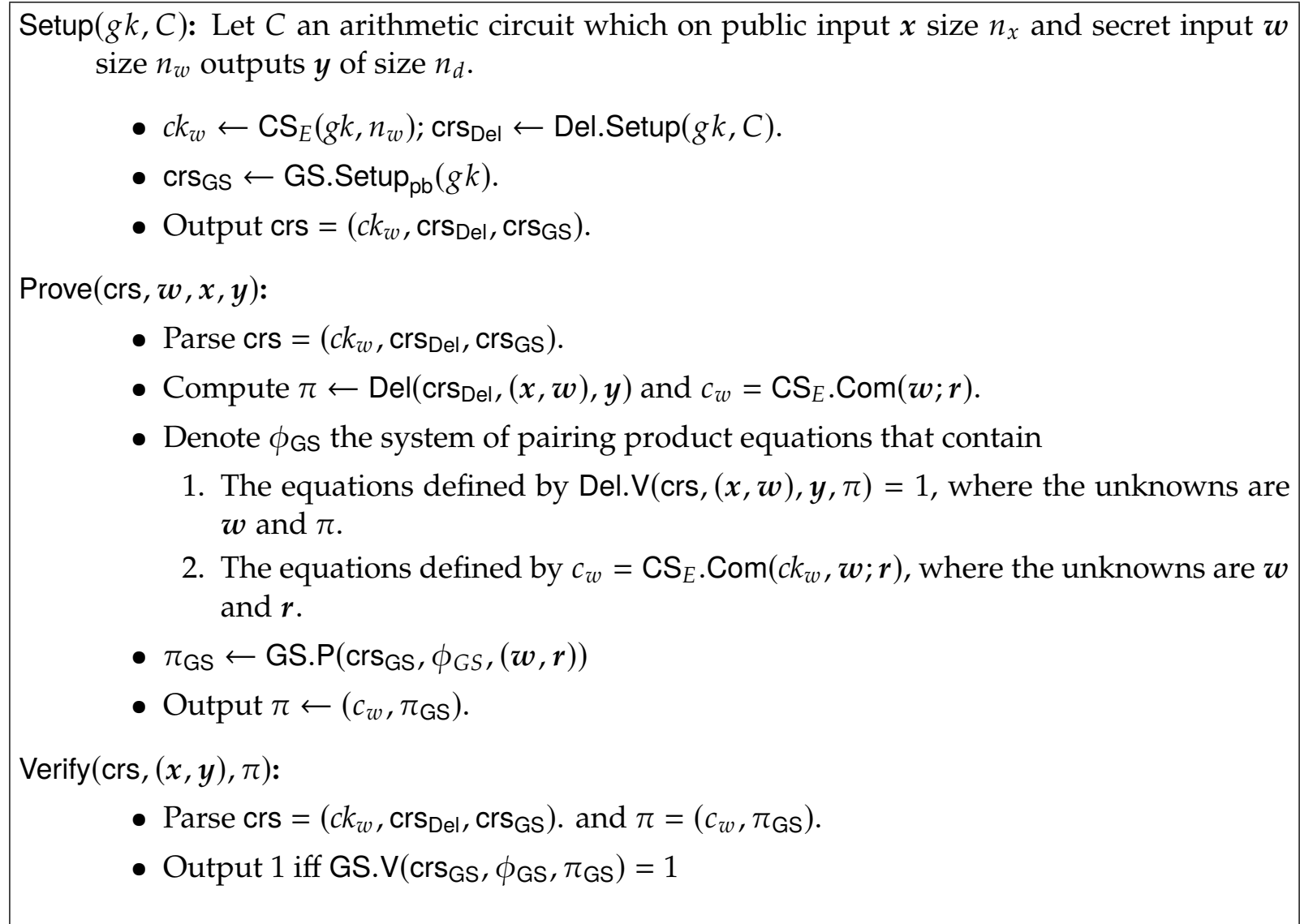

Figure 9: NIZK argument of NP. $\mathrm{CS}_{E}$ is an algebraic commitment, GS is the Groth-Sahai proof system of [GS08] and Del the delegation scheme of Fig. 8.

Theorem 17. Let $\mathrm{CS}_{E}$ be an algebraic commitment scheme that is hiding and extractable, GS the Groth-Sahai proof system of [GS08] and Del the delegation scheme of Fig. 8. Then, construction of Fig. 9 is a NIZK argument of knowledge. Furthermore, for every adversary $\mathcal{A}$ against knowledge soundness there exist adversaries $\mathcal{B}_{1}, \mathcal{B}_{2}$ against extractability of $\mathrm{CS}_{E}$ and against soundness of Del respectively such that $\operatorname{Adv}(\mathcal{A}) \leq \operatorname{Adv}_{\text {ext }}^{C S_{E}}\left(\mathcal{B}_{1}\right)+\operatorname{Adv}_{\text {snd }}^{\text {Del }}\left(\mathcal{B}_{2}\right)$.

Proof. Completeness follows by the correctness of $\mathrm{CS}_{E}$, and completeness of GS, Del. Computational zero knowledge follows from the computational zero-knowledge of GS and the hiding property of $\mathrm{CS}_{E}$. For knowledge soundness, we show how we can extract a valid witness given an accepting proof. In what follows, let $\mathcal{E}_{C S}$ be the extractors for $\mathrm{CS}_{E}$. The NIZK extractor $\mathcal{E}_{\mathcal{A}}\left(\mathrm{crs}, \boldsymbol{x}, \boldsymbol{y}, \pi=\left(c_{w}, \pi_{\mathrm{GS}}\right)\right)$ simply outputs $(\boldsymbol{w}, \pi) \leftarrow \mathcal{E}_{\mathrm{CS}}\left(c_{w}, c_{w}\right)$. Now, we claim that this a valid witness except with negligible probability. It is enough to note that if it is not, there are three possible cases:

1. The extractor $\mathcal{E}_{C S}$ failed which contradicts extractability of $\mathrm{CS}_{E}$.

2. The extracted solutions $w, \pi, r$ are not solutions to $\phi_{\mathrm{GS}}$, contradicting perfect soundness of GS since the proof verifies.

3. $y \neq C(x \| w)$. We can extract the solution $w, \pi, r$ and it must hold that

$$
\operatorname{Del} . \operatorname{Verify}(\operatorname{crs},(x, w), y, \pi)=1
$$

contradicting adaptive soundness of Del. 
As for efficiency, and specifically proof size, noting that the Groth-Sahai proof gives only a constant, multiplicative overhead to the proof -which is constant -, its size is dominated by the size of $\mathrm{CS}_{E}$. Depending on the choice of $\mathrm{CS}_{E}$ we can get qualitatively different constructions. We discuss the following cases:

(i) For a NIZK argument of knowledge under falsifiable assumptions, we can extend our result to apply to boolean circuits instead of arithmetic ones by arithmetizing the different types of gates e.g. as in[DFGK14]. We can then use commitments for boolean vectors that are extractable in the field under falsifiable assumptions such as Groth-Sahai commitments or using methods of [GHR15b]. The proof size in this case is $O(\lambda|w|)$ where $w$ is the secret input. Since fully succinct algebraic extractable commitments that allow extraction in the field are unknown to exist under falsifiable assumptions, we cannot achieve a (concretely more efficient) NIZK AoK for arithmetic circuits.

(ii) We use succinct extractable commitments based on knowledge assumptions, yielding a SNARK of constant proof size. Additionally, since the committed value is the secret input and not the full wire assignment we get a quantitatively smaller assumption size. For example, in case of $q$-power knowledge of exponent assumption ( $q$-KEA) used in [DFGK14], we use only the $n_{w}$-KEA while [DFGK14] requires the larger (and hence stronger) $|C|$-KEA.

(iii) To construct a compact NIZK where the proof size is $O(|w|)+\operatorname{poly}(\kappa)$ we follow essentially the ideas of [KNYY19; KNYY20]. We use a secret key symmetric encryption scheme SE = (KGen, Enc, Dec) with additive overhead in the cyphertexts. That is, $\mid \operatorname{SE}$. Enc $(s k, w) \mid=$ $O(|\boldsymbol{w}|)+\operatorname{poly}(\kappa)$. We use the NIZK from figure 9, instantiated with the commitment scheme from (i), for showing knowledge of some $K \in \operatorname{Im}(S E . K G e n)$ such that $C^{\prime}(K, D)=1$, where $K$ is the secret input, $D$ the public input, and $C^{\prime}(K, D)=C(\operatorname{SE} \cdot \operatorname{Dec}(K, D))$. To prove that $C(w)=1$ the prover picks $K \leftarrow$ SE.KGen $\left(1^{K}\right)$ and computes $D \leftarrow \operatorname{SE} . \operatorname{Enc}(K, w)$ together with a proof $\pi$ that $C^{\prime}(K, D)=1$. The verifier on input crs, $D$ and $\pi$ ouputs 1 if $\pi$ is a valid proof for $D$. In spite of [KNYY19; KNYY20] and by the nature of the underlying non-compact NIZK scheme we use, we don't require SE.Dec to be in $\mathrm{NC}^{1}$.

\section{Acknowledgements}

We thank Carla Ráfols for various discussions and comments on this work. We also thank the anonymous reviewers of TCC 21 for their useful comments. 


\section{References}

[ACC+16] Prabhanjan Ananth, Yu-Chi Chen, Kai-Min Chung, Huijia Lin, and Wei-Kai Lin. "Delegating RAM Computations with Adaptive Soundness and Privacy". In: TCC 2016-B, Part II. Ed. by Martin Hirt and Adam D. Smith. Vol. 9986. LNCS. Springer, Heidelberg, Oct. 2016, pp. 3-30. Dor: 10 . 1007/978-3-662-53644-5_1.

[AFG+10] Masayuki Abe, Georg Fuchsbauer, Jens Groth, Kristiyan Haralambiev, and Miyako Ohkubo. "Structure-Preserving Signatures and Commitments to Group Elements". In: CRYPTO 2010. Ed. by Tal Rabin. Vol. 6223. LNCS. Springer, Heidelberg, Aug. 2010, pp. 209-236. DOr: 10.1007/978-3-642-14623-7_12.

[AFG+16] Masayuki Abe, Georg Fuchsbauer, Jens Groth, Kristiyan Haralambiev, and Miyako Ohkubo. "Structure-Preserving Signatures and Commitments to Group Elements". In: Journal of Cryptology 29.2 (Apr. 2016), pp. 363-421. DOI: 10.1007/s00145-0149196- 7.

[BBS04] Dan Boneh, Xavier Boyen, and Hovav Shacham. "Short Group Signatures". In: CRYPTO 2004. Ed. by Matthew Franklin. Vol. 3152. LNCS. Springer, Heidelberg, Aug. 2004, pp. 41-55. Dor: 10.1007/978-3-540-28628-8_3.

[BCL+21] Benedikt Bünz, Alessandro Chiesa, William Lin, Pratyush Mishra, and Nicholas Spooner. "Proof-Carrying Data Without Succinct Arguments". In: CRYPTO 2021, Part I. Ed. by Tal Malkin and Chris Peikert. Vol. 12825. LNCS. Virtual Event: Springer, Heidelberg, Aug. 2021, pp. 681-710. Dor: 10. 1007/978-3-030-84242Q_24.

[BCMS20] Benedikt Bünz, Alessandro Chiesa, Pratyush Mishra, and Nicholas Spooner. "Recursive Proof Composition from Accumulation Schemes". In: TCC 2020, Part II. Ed. by Rafael Pass and Krzysztof Pietrzak. Vol. 12551. LNCS. Springer, Heidelberg, Nov. 2020, pp. 1-18. DoI: 10. 1007/978-3-030-64378-2_1.

[BDH+19] Michael Backes, Nico Döttling, Lucjan Hanzlik, Kamil Kluczniak, and Jonas Schneider. "Ring Signatures: Logarithmic-Size, No Setup - from Standard Assumptions". In: EUROCRYPT 2019, Part III. Ed. by Yuval Ishai and Vincent Rijmen. Vol. 11478. LNCS. Springer, Heidelberg, May 2019, pp. 281-311. Dor: 10.1007/978-3-030-17659-4_10.

[BGL+15] Nir Bitansky, Sanjam Garg, Huijia Lin, Rafael Pass, and Sidharth Telang. Succinct Randomized Encodings and their Applications. Cryptology ePrint Archive, Report 2015/356. https: //eprint.iacr. org/2015/356. 2015.

[BHK17] Zvika Brakerski, Justin Holmgren, and Yael Tauman Kalai. “Non-interactive delegation and batch NP verification from standard computational assumptions". In: 49th ACM STOC. Ed. by Hamed Hatami, Pierre McKenzie, and Valerie King. ACM Press, June 2017, pp. 474-482. Dor: 10.1145/3055399. 3055497.

[BKK+18] Saikrishna Badrinarayanan, Yael Tauman Kalai, Dakshita Khurana, Amit Sahai, and Daniel Wichs. "Succinct delegation for low-space non-deterministic computation". In: 50th ACM STOC. Ed. by Ilias Diakonikolas, David Kempe, and Monika Henzinger. ACM Press, June 2018, pp. 709-721. Dor: 10.1145/3188745 . 3188924.

[CCC+16] Yu-Chi Chen, Sherman S. M. Chow, Kai-Min Chung, Russell W. F. Lai, Wei-Kai Lin, and Hong-Sheng Zhou. "Cryptography for Parallel RAM from Indistinguishability Obfuscation". In: ITCS 2016. Ed. by Madhu Sudan. ACM, Jan. 2016, pp. 179-190. Dor: 10.1145/2840728.2840769. 
[CCH+19] Ran Canetti, Yilei Chen, Justin Holmgren, Alex Lombardi, Guy N. Rothblum, Ron D. Rothblum, and Daniel Wichs. "Fiat-Shamir: from practice to theory". In: 51st ACM STOC. Ed. by Moses Charikar and Edith Cohen. ACM Press, June 2019, pp. 1082-1090. Dor: 10.1145/3313276.3316380.

[CHJV15] Ran Canetti, Justin Holmgren, Abhishek Jain, and Vinod Vaikuntanathan. "Succinct Garbling and Indistinguishability Obfuscation for RAM Programs". In: 47th ACM STOC. Ed. by Rocco A. Servedio and Ronitt Rubinfeld. ACM Press, June 2015, pp. 429-437. DOr: 10.1145/2746539.2746621.

[CS02] Ronald Cramer and Victor Shoup. "Universal Hash Proofs and a Paradigm for Adaptive Chosen Ciphertext Secure Public-Key Encryption". In: EUROCRYPT 2002. Ed. by Lars R. Knudsen. Vol. 2332. LNCS. Springer, Heidelberg, Apr. 2002, pp. 4564. DOI: $10.1007 / 3-540-46035-7 \_4$.

[DFGK14] George Danezis, Cédric Fournet, Jens Groth, and Markulf Kohlweiss. "Square Span Programs with Applications to Succinct NIZK Arguments". In: ASIACRYPT 2014, Part I. Ed. by Palash Sarkar and Tetsu Iwata. Vol. 8873. LNCS. Springer, Heidelberg, Dec. 2014, pp. 532-550. Dor: 10.1007/978-3-662-45611-8_28.

[DGP+19] Vanesa Daza, Alonso González, Zaira Pindado, Carla Ràfols, and Javier Silva. "Shorter Quadratic QA-NIZK Proofs". In: PKC 2019, Part I. Ed. by Dongdai Lin and Kazue Sako. Vol. 11442. LNCS. Springer, Heidelberg, Apr. 2019, pp. 314-343. DoI: $10.1007 / 978-3-030-17253-4 \_11$.

[EG14] Alex Escala and Jens Groth. "Fine-Tuning Groth-Sahai Proofs". In: PKC 2014. Ed. by Hugo Krawczyk. Vol. 8383. LNCS. Springer, Heidelberg, Mar. 2014, pp. 630649. DoI: 10.1007/978-3-642-54631-0_36.

[EHK+13] Alex Escala, Gottfried Herold, Eike Kiltz, Carla Ràfols, and Jorge Villar. "An Algebraic Framework for Diffie-Hellman Assumptions". In: CRYPTO 2013, Part II. Ed. by Ran Canetti and Juan A. Garay. Vol. 8043. LNCS. Springer, Heidelberg, Aug. 2013, pp. 129-147. Dor: 10.1007/978-3-642-40084-1_8.

[FLPS20] Prastudy Fauzi, Helger Lipmaa, Zaira Pindado, and Janno Siim. Somewhere Statistically Binding Commitment Schemes with Applications. Cryptology ePrint Archive, Report 2020/652. https://eprint.iacr.org/2020/652. 2020.

[GGPR13] Rosario Gennaro, Craig Gentry, Bryan Parno, and Mariana Raykova. “Quadratic Span Programs and Succinct NIZKs without PCPs". In: EUROCRYPT 2013. Ed. by Thomas Johansson and Phong Q. Nguyen. Vol. 7881. LNCS. Springer, Heidelberg, May 2013, pp. 626-645. Dor: 10.1007/978-3-642-38348-9_37.

[GHR15a] Alonso González, Alejandro Hevia, and Carla Ràfols. QA-NIZK Arguments in Asymmetric Groups: New Tools and New Constructions. Cryptology ePrint Archive, Report 2015/910. https://eprint.iacr.org/2015/910. 2015.

[GHR15b] Alonso González, Alejandro Hevia, and Carla Ràfols. "QA-NIZK Arguments in Asymmetric Groups: New Tools and New Constructions". In: ASIACRYPT 2015, Part I. Ed. by Tetsu Iwata and Jung Hee Cheon. Vol. 9452. LNCS. Springer, Heidelberg, Nov. 2015, pp. 605-629. DoI: 10. 1007/978-3-662-48797-6_25.

[GKR08] Shafi Goldwasser, Yael Tauman Kalai, and Guy N. Rothblum. “Delegating computation: interactive proofs for muggles". In: 40th ACM STOC. Ed. by Richard E. Ladner and Cynthia Dwork. ACM Press, May 2008, pp. 113-122. Dor: 10.1145/ 1374376.1374396. 
[GOS06] Jens Groth, Rafail Ostrovsky, and Amit Sahai. "Perfect Non-interactive Zero Knowledge for NP". In: EUROCRYPT 2006. Ed. by Serge Vaudenay. Vol. 4004. LNCS. Springer, Heidelberg, May 2006, pp. 339-358. Dor: 10. 1007/11761679_21.

[GR16] Alonso González and Carla Ràfols. "New Techniques for Non-interactive Shuffle and Range Arguments". In: ACNS 16. Ed. by Mark Manulis, Ahmad-Reza Sadeghi, and Steve Schneider. Vol. 9696. LNCS. Springer, Heidelberg, June 2016, pp. 427-444. Dor: 10.1007/978-3-319-39555-5_23.

[GR19] Alonso González and Carla Ràfols. "Shorter Pairing-Based Arguments Under Standard Assumptions". In: ASIACRYPT 2019, Part III. Ed. by Steven D. Galbraith and Shiho Moriai. Vol. 11923. LNCS. Springer, Heidelberg, Dec. 2019, pp. 728757. DOI: $10.1007 / 978-3-030-34618-8 \_25$.

[Gro16] Jens Groth. “On the Size of Pairing-Based Non-interactive Arguments". In: EUROCRYPT 2016, Part II. Ed. by Marc Fischlin and Jean-Sébastien Coron. Vol. 9666. LNCS. Springer, Heidelberg, May 2016, pp. 305-326. Dor: 10 . 1007/978-3-66249896-5_11.

[GS08] Jens Groth and Amit Sahai. "Efficient Non-interactive Proof Systems for Bilinear Groups". In: EUROCRYPT 2008. Ed. by Nigel P. Smart. Vol. 4965. LNCS. Springer, Heidelberg, Apr. 2008, pp. 415-432. Dor: 10.1007/978-3-540-78967-3_24.

[GW11] Craig Gentry and Daniel Wichs. "Separating succinct non-interactive arguments from all falsifiable assumptions". In: 43rd ACM STOC. Ed. by Lance Fortnow and Salil P. Vadhan. ACM Press, June 2011, pp. 99-108. Dor: 10.1145/1993636. 1993651.

[HW15] Pavel Hubacek and Daniel Wichs. "On the Communication Complexity of Secure Function Evaluation with Long Output". In: ITCS 2015. Ed. by Tim Roughgarden. ACM, Jan. 2015, pp. 163-172. DOI: 10.1145/2688073.2688105.

[JKKZ20] Ruta Jawale, Yael Tauman Kalai, Dakshita Khurana, and Rachel Zhang. SNARGs for Bounded Depth Computations and PPAD Hardness from Sub-Exponential LWE. Cryptology ePrint Archive, Report 2020/980. https://eprint.iacr . org/2020/ 980. 2020.

[JR13] CharanjitS. Jutla and Arnab Roy. "Shorter Quasi-Adaptive NIZK Proofs for Linear Subspaces". In: ASIACRYPT 2013, Part I. Ed. by Kazue Sako and Palash Sarkar. Vol. 8269. LNCS. Springer, Heidelberg, Dec. 2013, pp. 1-20. Dor: 10 . 1007/978-3642-42033-7_1.

[JR14] Charanjit S. Jutla and Arnab Roy. "Switching Lemma for Bilinear Tests and Constant-Size NIZK Proofs for Linear Subspaces". In: CRYPTO 2014, Part II. Ed. by Juan A. Garay and Rosario Gennaro. Vol. 8617. LNCS. Springer, Heidelberg, Aug. 2014, pp. 295-312. Dor: 10.1007/978-3-662-44381-1_17.

[KLW15] Venkata Koppula, Allison Bishop Lewko, and Brent Waters. "Indistinguishability Obfuscation for Turing Machines with Unbounded Memory". In: 47th ACM STOC. Ed. by Rocco A. Servedio and Ronitt Rubinfeld. ACM Press, June 2015, pp. 419-428. Dor: 10.1145/2746539.2746614.

[KNYY19] Shuichi Katsumata, Ryo Nishimaki, Shota Yamada, and Takashi Yamakawa. "Exploring Constructions of Compact NIZKs from Various Assumptions". In: CRYPTO 2019, Part III. Ed. by Alexandra Boldyreva and Daniele Micciancio. Vol. 11694. LNCS. Springer, Heidelberg, Aug. 2019, pp. 639-669. Dor: $10.1007 /$ 978-3-030-26954-8_21. 
[KNYY20] Shuichi Katsumata, Ryo Nishimaki, Shota Yamada, and Takashi Yamakawa. "Compact NIZKs from Standard Assumptions on Bilinear Maps". In: EUROCRYPT 2020, Part III. Ed. by Anne Canteaut and Yuval Ishai. Vol. 12107. LNCS. Springer, Heidelberg, May 2020, pp. 379-409. Dor: 10.1007/978-3-030-457273_13.

[KP16] Yael Tauman Kalai and Omer Paneth. "Delegating RAM Computations". In: TCC 2016-B, Part II. Ed. by Martin Hirt and Adam D. Smith. Vol. 9986. LNCS. Springer, Heidelberg, Oct. 2016, pp. 91-118. Dor: 10 . 1007/978-3-662-536445_4.

[KPY18] Yael Kalai, Omer Paneth, and Lisa Yang. On Publicly Verifiable Delegation From Standard Assumptions. Cryptology ePrint Archive, Report 2018/776. https : / / eprint. iacr . org/2018/776. 2018.

[KPY19] Yael Tauman Kalai, Omer Paneth, and Lisa Yang. "How to delegate computations publicly". In: 51st ACM STOC. Ed. by Moses Charikar and Edith Cohen. ACM Press, June 2019, pp. 1115-1124. Dor: 10.1145/3313276.3316411.

[KRR13] Yael Tauman Kalai, Ran Raz, and Ron D. Rothblum. “Delegation for bounded space". In: 45th ACM STOC. Ed. by Dan Boneh, Tim Roughgarden, and Joan Feigenbaum. ACM Press, June 2013, pp. 565-574. Dor: 10. 1145/2488608 . 2488679.

[KRR14] Yael Tauman Kalai, Ran Raz, and Ron D. Rothblum. "How to delegate computations: the power of no-signaling proofs". In: 46th ACM STOC. Ed. by David B. Shmoys. ACM Press, May 2014, pp. 485-494. Dor: 10.1145/2591796.2591809.

[KW15] Eike Kiltz and Hoeteck Wee. “Quasi-Adaptive NIZK for Linear Subspaces Revisited". In: EUROCRYPT 2015, Part II. Ed. by Elisabeth Oswald and Marc Fischlin. Vol. 9057. LNCS. Springer, Heidelberg, Apr. 2015, pp. 101-128. Dor: 10 . 1007/9783-662-46803-6_4.

[LPJY13] Benoît Libert, Thomas Peters, Marc Joye, and Moti Yung. "Linearly Homomorphic Structure-Preserving Signatures and Their Applications". In: CRYPTO 2013, Part II. Ed. by Ran Canetti and Juan A. Garay. Vol. 8043. LNCS. Springer, Heidelberg, Aug. 2013, pp. 289-307. Dor: 10. 1007/978-3-642-40084-1_17.

[LPJY14] Benoît Libert, Thomas Peters, Marc Joye, and Moti Yung. "Non-malleability from Malleability: Simulation-Sound Quasi-Adaptive NIZK Proofs and CCA2-Secure Encryption from Homomorphic Signatures". In: EUROCRYPT 2014. Ed. by Phong Q. Nguyen and Elisabeth Oswald. Vol. 8441. LNCS. Springer, Heidelberg, May 2014, pp. 514-532. DOI: 10.1007/978-3-642-55220-5_29.

[MRV16] Paz Morillo, Carla Ràfols, and Jorge Luis Villar. "The Kernel Matrix DiffieHellman Assumption". In: ASIACRYPT 2016, Part I. Ed. by Jung Hee Cheon and Tsuyoshi Takagi. Vol. 10031. LNCS. Springer, Heidelberg, Dec. 2016, pp. 729-758. DOI: $10.1007 / 978-3-662-53887-6 \_27$.

[OPWW15] Tatsuaki Okamoto, Krzysztof Pietrzak, Brent Waters, and Daniel Wichs. “New Realizations of Somewhere Statistically Binding Hashing and Positional Accumulators". In: ASIACRYPT 2015, Part I. Ed. by Tetsu Iwata and Jung Hee Cheon. Vol. 9452. LNCS. Springer, Heidelberg, Nov. 2015, pp. 121-145. Dor: 10 . 1007 /9783-662-48797-6_6. 
[PR17] Omer Paneth and Guy N. Rothblum. "On Zero-Testable Homomorphic Encryption and Publicly Verifiable Non-interactive Arguments". In: TCC 2017, Part II. Ed. by Yael Kalai and Leonid Reyzin. Vol. 10678. LNCS. Springer, Heidelberg, Nov. 2017, pp. 283-315. Dor: 10.1007/978-3-319-70503-3_9.

[RRR16] Omer Reingold, Guy N. Rothblum, and Ron D. Rothblum. "Constant-round interactive proofs for delegating computation". In: 48th ACM STOC. Ed. by Daniel Wichs and Yishay Mansour. ACM Press, June 2016, pp. 49-62. Dor: 10. 1145 / 2897518.2897652.

[RS20] Carla Ràfols and Javier Silva. QA-NIZK Arguments of Same Opening for Bilateral Commitments. Cryptology ePrint Archive, Report 2020/569. https: / / eprint . iacr . org/2020/569. 2020.

[Vil12] Jorge Luis Villar. "Optimal Reductions of Some Decisional Problems to the Rank Problem". In: ASIACRYPT 2012. Ed. by Xiaoyun Wang and Kazue Sako. Vol. 7658. LNCS. Springer, Heidelberg, Dec. 2012, pp. 80-97. Dor: 10 . 1007 /978-3-64234961-4_7. 
since $\mathbf{B}, \mathbf{D}$ are uniform conditioned on their sum being equal to

$$
\begin{aligned}
\mathbf{B}+\mathbf{D} & =\left(\left(\mathbf{M}^{\top}+\mathbf{N}^{\top}\right) \mathbf{K}_{1,1}+\mathbf{P}^{\top} \mathbf{K}_{3,1}, \boldsymbol{s}+\mathbf{P}^{\top} \mathbf{K}_{3,2}\right)+\left(\mathbf{R}^{\top} \mathbf{K}_{1,1}, \boldsymbol{t}\right)+\boldsymbol{\Gamma}-\mathbf{\Gamma} \\
& =\left(\left(\mathbf{M}^{\top}+\mathbf{N}^{\top}\right) \mathbf{K}_{1,1}+\mathbf{P}^{\top} \mathbf{K}_{3,1},\left(\mathbf{M}^{\top}+\mathbf{N}^{\top}\right) \mathbf{K}_{1,2}+\mathbf{P}^{\top} \mathbf{K}_{3,2}\right) \\
& =\left(\mathbf{M}^{\top}+\mathbf{N}^{\top}\right)\left(\begin{array}{l}
\mathbf{K}_{1,1} \\
\mathbf{K}_{1,2}
\end{array}\right)+\mathbf{P}^{\top}\left(\begin{array}{l}
\mathbf{K}_{3,1} \\
\mathbf{K}_{3,2}
\end{array}\right)=\left(\mathbf{M}^{\top}+\mathbf{N}^{\top} \mid \mathbf{P}^{\top}\right) \mathbf{K}, \\
\mathbf{K A} & =\left(\begin{array}{ll}
\left(\mathbf{C}_{1}-\mathbf{K}_{1,2} \underline{\mathbf{A}}\right) \overline{\mathbf{A}}^{-1} & \mathbf{K}_{1,2} \\
\left(\mathbf{C}_{3}-\mathbf{K}_{3,2} \underline{\mathbf{A}}\right) \overline{\mathbf{A}}^{-1} & \mathbf{K}_{3,2}
\end{array}\right)\left(\begin{array}{l}
\overline{\mathbf{A}} \\
\mathbf{A}
\end{array}\right)=\left(\begin{array}{l}
\mathbf{C}_{1}-\mathbf{K}_{1,2} \mathbf{A}+\mathbf{K}_{1,2} \underline{\mathbf{A}} \\
\mathbf{C}_{3}-\mathbf{K}_{3,1} \underline{\mathbf{A}}+\mathbf{K}_{3,2} \underline{\mathbf{A}}
\end{array}\right)=\mathbf{C},
\end{aligned}
$$

and by definition $\boldsymbol{\pi}+\boldsymbol{\theta}=\left(\boldsymbol{d}^{\top}\left(\mathbf{C}_{3}-\mathbf{K}_{3,2} \underline{\mathbf{A}}\right) \overline{\mathbf{A}}^{-1}, \boldsymbol{d}^{\top} \mathbf{K}_{3,2}\right)=\left(\boldsymbol{d}^{\top} \mathbf{K}_{3,1}, \boldsymbol{d}^{\top} \mathbf{K}_{3,2}\right)=\boldsymbol{d}^{\top} \mathbf{K}_{3}$.

Therefore we just need to argue that the distribution of $\mathbf{K}$ is the same in both games. But this is an immediate consequence of the fact that for every value of $\left(\mathbf{C}, \mathbf{K}_{1,1}, \mathbf{K}_{3,1}\right)$ there exists a unique value of $\left(\mathbf{K}_{1,2}, \mathbf{K}_{3,2}\right)$ which is compatible with $\mathbf{C}=\mathbf{K A}$. Indeed, $\mathbf{C}=\mathbf{K A} \Longleftrightarrow \mathbf{C}_{i}=$ $\mathbf{K}_{i, 1} \overline{\mathbf{A}}+\mathbf{K}_{i, 2} \underline{\mathbf{A}}, i=1,3 \Longleftrightarrow\left(\mathbf{C}_{i}-\mathbf{K}_{i, 2} \underline{\mathbf{A}}\right) \overline{\mathbf{A}}^{-1}=\mathbf{K}_{i, 1}, i=1,3$.

Lemma 7. For any PPT algorithm $\mathcal{A}$ there exists a PPT algorithm $\mathcal{B}$ such that

$$
\operatorname{Adv}_{\prod_{k t-s u m, h^{\prime}}}(\mathcal{A}) \leq \operatorname{Adv}_{\left(\mathcal{M}^{\top}, \mathcal{N}^{\top}, h\right)-\mathrm{MDDH}}(\mathcal{B}) .
$$

Proof. We construct an adversary $\mathcal{B}$ that receives the challenge $\left(\left[\mathbf{M}^{\top}\right]_{1},\left[\mathbf{N}^{\top}\right]_{2},\left[s^{*}\right]_{1},\left[t^{*}\right]_{2}, h\left(\left[\mathbf{M}^{\top}, \mathbf{N}^{\top}\right]\right)\right.$, where $\boldsymbol{s}^{*}+\boldsymbol{t}^{*}=\left(\mathbf{M}^{\top}+\mathbf{N}^{\top}\right) \boldsymbol{w}, \boldsymbol{w} \leftarrow \mathbb{Z}_{p}^{\ell_{1}}$, or $\boldsymbol{s}^{*}, \boldsymbol{t}^{*} \leftarrow \mathbb{Z}_{p}^{n}$. $\mathcal{B}$ computes the crs running $\mathrm{K}^{*}\left(g k,[\mathbf{M}]_{1},[\mathbf{N}]_{2},[\mathbf{P}]_{1}\right)$ but replaces $[s]_{1},[\boldsymbol{t}]_{2}$ with $\left[\boldsymbol{s}^{*}\right]_{1},\left[\boldsymbol{t}^{*}\right]_{2}$ respectively, and then runs $\mathcal{A}$ as in game Game ${ }_{1}$. Since Game ${ }_{1}$ corresponds to the first case and Game 2 to the second, the lemma follows.

Lemma 8. For any (unbounded) algorithm $\mathcal{A}, \operatorname{Pr}\left[\operatorname{Game}_{2}(\mathcal{A})=1\right] \leq 1 / p$.

Proof. We will show that, conditioned on $\mathbf{A}, \mathbf{C}, \mathbf{B}+\mathbf{D}, \mathbf{M}+\mathbf{N}, \mathbf{P}$, the matrix $\mathbf{K}_{3,2}$ is uniformly distributed. Since it holds that $(\mathbf{B}+\mathbf{D}) \mathbf{A}=\left(\mathbf{M}^{\top}+\mathbf{N}^{\top} \mid \mathbf{P}^{\top}\right) \mathbf{C}$, we get that the first $k$ columns of $\mathbf{B}+\mathbf{D}$, namely $\mathbf{B}_{1}+\mathbf{D}_{1}$, are completely determined by the last columns $\mathbf{B}_{2}+\mathbf{D}_{2}$. Indeed

$$
\left(\mathbf{B}_{1}+\mathbf{D}_{1}, \mathbf{B}_{2}+\mathbf{D}_{2}\right) \mathbf{A}=\left(\mathbf{M}^{\top}+\mathbf{N}^{\top} \mid \mathbf{P}^{\top}\right) \mathbf{C} \Longleftrightarrow \mathbf{B}_{1}+\mathbf{D}_{1}=\left(\left(\mathbf{M}^{\top}+\mathbf{N}^{\top} \mid \mathbf{P}^{\top}\right) \mathbf{C}-\left(\mathbf{B}_{2}+\mathbf{D}_{2}\right) \underline{\mathbf{A}}\right) \overline{\mathbf{A}}^{-1} .
$$

Hence, conditioning in $\mathbf{A}, \mathbf{C}, \mathbf{B}_{1}+\mathbf{D}_{1}, \mathbf{M}+\mathbf{N}, \mathbf{P}$ doesn't alter the probability. We have that $\mathbf{B}_{2}+\mathbf{D}_{2}=(s+\boldsymbol{t})+\mathbf{P}^{\top} \mathbf{K}_{3,2}$, which consists of $n$ equations on $n+\ell_{2}$ variables. It follows that there are $\ell_{2}$ free variables. Then $\mathbf{K}_{3,2}$ is uniformly distributed and hence completely hidden to the adversary.

Note that

$$
\pi+\boldsymbol{\theta}=\boldsymbol{d}^{\top} \mathrm{K}_{3} \Longrightarrow \pi_{2}+\boldsymbol{\theta}_{2}=\boldsymbol{d}^{\top} \mathbf{K}_{3,2},
$$

where $\pi_{2}, \theta_{2}$ are the last element of $\pi, \theta$ respectively. Given that $d \neq 0$, the last equation only holds with probability $1 / p$ and so $\mathcal{A}$ 's probability of winning.

The knowledge transfer property is a direct consequence of Lemma 5. We present the proof next.

Theorem 18. For any adversary $\mathcal{A}$ against the soundness of $\Pi_{\mathrm{kt}-\mathrm{sum}}$ with respect to $\mathcal{L}_{\text {sum }}^{\text {no }}$ there exist adversaries $\mathcal{B}_{1}$ and $\mathcal{B}_{2}$ such that

$$
\operatorname{Adv}_{\mathrm{kt}-\operatorname{sum}}(\mathcal{A}) \leq \operatorname{Adv}_{\mathcal{D}_{k}-\operatorname{SKerMDH}}\left(\mathcal{B}_{1}\right)+\operatorname{Adv}_{\left(\mathcal{M}^{\top}, \mathcal{N}^{\top}, h\right)-\mathrm{MDDH}}+1 / p .
$$


Proof. Given an adversary that produces a valid proof for a statement in $\mathcal{L}_{\text {sum }}^{\text {no }}$, successful attacks can be divided in two categories.

Type I: In this attack $\pi+\boldsymbol{\theta} \neq\left(\boldsymbol{c}_{1}^{\top}+\boldsymbol{c}_{2}^{\top}\right) \mathbf{K}_{1}+\boldsymbol{d}^{\top} \mathbf{K}_{3}$.

Type II: In this type of attack $\pi+\boldsymbol{\theta}=\left(c_{1}^{\top}+c_{2}^{\top}\right) \mathbf{K}_{1}+d^{\top} \mathbf{K}_{3}$.

Type I attacks are computationally infeasible when $\bar{k}=k+1$, as they can be used to construct an adversary $\mathcal{B}_{1}$ against the $\mathcal{D}_{k}$-SKerMDH assumption. ${ }^{21}$ Adversary $\mathcal{B}_{1}$ receives a challenge $[\mathbf{A}]_{1,2}$ and then runs the soundness experiment for $\mathcal{A}$. When $\mathcal{A}$ outputs $\left(\left[\boldsymbol{c}_{1}\right]_{1},\left[\boldsymbol{c}_{2}\right]_{2},[\boldsymbol{d}]_{1},[\pi]_{1},[\boldsymbol{\theta}]_{2}\right)$, $\mathcal{B}_{1}$ outputs $\left[\boldsymbol{\pi}^{\dagger}\right]_{1}=[\pi]_{1}-\left[\boldsymbol{c}_{1}^{\top}\right]_{1} \mathbf{K}_{1}-\left[\boldsymbol{d}^{\top}\right]_{1} \mathbf{K}_{3},\left[\boldsymbol{\theta}^{\dagger}\right]_{2}=[\boldsymbol{\theta}]_{1}-\left[\boldsymbol{c}_{2}^{\top}\right]_{1} \mathbf{K}_{1}$ where it holds that $\boldsymbol{\pi}+\boldsymbol{\theta} \neq\left(\boldsymbol{c}_{1}^{\top}+\boldsymbol{c}_{2}^{\top}\right) \mathbf{K}_{1}+\boldsymbol{d}^{\top} \mathbf{K}_{3}$. Since $[\boldsymbol{\pi}]_{1},[\boldsymbol{\theta}]_{2}$ is accepted by the verifier we get that $e\left([\boldsymbol{\pi}]_{1},[\mathbf{A}]_{2}\right)+$ $e\left([\boldsymbol{\theta}]_{2},[\mathbf{A}]_{1}\right)=e\left(\left[\boldsymbol{c}_{1}^{\top}\right]_{1},\left[\mathbf{C}_{1}\right]_{2}\right)+e\left(\left[\boldsymbol{c}_{2}^{\top}\right]_{2},\left[\mathbf{C}_{1}\right]_{1}\right)+e\left(\left[\boldsymbol{d}^{\top}\right]_{1},\left[\mathbf{C}_{3}\right]_{2}\right)$ and then $\left(\boldsymbol{\pi}^{\dagger}+\boldsymbol{\theta}^{\dagger}\right) \mathbf{A}=(\boldsymbol{\pi}+\boldsymbol{\theta}) \mathbf{A}-$ $\left(c_{1}^{\top}+c_{2}^{\top}\right) \mathbf{K}_{1} \mathbf{A}-\boldsymbol{d}^{\top} \mathbf{K}_{3} \mathbf{A}=(\boldsymbol{\pi}+\boldsymbol{\theta}) \mathbf{A}-\left(c_{1}+c_{2}\right)^{\top} \mathbf{C}_{1}-\boldsymbol{d}^{\top} \mathbf{C}_{3}=0$. We conclude that the success probability of a type I attack is bounded by $\operatorname{Adv}_{\mathcal{D}_{k} \text {-SKerMDH }}\left(\mathcal{B}_{1}\right)$.

For type II attacks, since $[\pi]_{1}=\left[c_{1}^{\top}\right]_{1} \mathbf{K}_{1}+\left[\boldsymbol{d}^{\top}\right]_{1} \mathbf{K}_{3},[\boldsymbol{\theta}]_{2}=\left[\boldsymbol{c}_{2}^{\top}\right]_{2} \mathbf{K}_{1}$ is a valid proof for $\left(\begin{array}{l}{\left[c_{1}\right]_{1}} \\ {\left[c_{2}\right]_{2}} \\ {[d]_{1}}\end{array}\right)$, then, by linearity of the verification equations $\pi^{\dagger}=\boldsymbol{\pi}-\boldsymbol{w}^{\top} \mathbf{B}$ and $\boldsymbol{\theta}^{\dagger}=\boldsymbol{\theta}-\boldsymbol{w}^{\top} \mathbf{B}$ is a valid proof for $\left(\begin{array}{c}0 \\ 0 \\ {\left[\boldsymbol{d}^{\dagger}\right]_{1}}\end{array}\right)=\left(\begin{array}{c}{\left[\boldsymbol{c}_{1}\right]_{1}-[\mathbf{M}]_{1} w} \\ {\left[\boldsymbol{c}_{2}\right]_{2}-[\mathbf{N}]_{2} w} \\ {[\boldsymbol{d}]_{1}-[\mathbf{P}]_{1} w}\end{array}\right)$. Since $\boldsymbol{d} \neq \mathbf{N} \boldsymbol{w}$, we conclude that an attacker of type II can be turned into an attacker $\mathcal{B}_{2}$ for Lemma 5.

We next note that the argument of knowledge transfer remains secure even for matrix distribution that also include some zero columns.

Theorem 19. Let $\mathcal{M}^{\prime}, \mathcal{N}^{\prime}, \mathcal{P}^{\prime}, Q^{\prime}$ be matrix distributions that sample $\left(\mathbf{M} \mid \mathbf{0}_{\ell_{1} \times n^{\prime}}\right)$, $\left(\mathbf{N} \mid \mathbf{0}_{\ell_{2} \times n^{\prime}}\right)$, $\left(\mathbf{P} \mid \mathbf{0}_{\ell_{3} \times n^{\prime}}\right),\left(\mathbf{Q} \mid \mathbf{0}_{\ell_{4} \times n^{\prime}}\right)$ where $\mathbf{M} \leftarrow \mathcal{M}, \mathbf{N} \leftarrow \mathcal{N}, \mathbf{P} \leftarrow \mathcal{P}, \mathbf{Q} \leftarrow \mathbf{Q}$.

1. For any adversary $\mathcal{A}$ against the $h$-strong soundness of $\Pi_{k t-l i n}$ there exist adversaries $\mathcal{B}_{1}$ and $\mathcal{B}_{2}$ such that $\operatorname{Adv}_{\Pi_{k-\text { lin } h^{\prime}}}(\mathcal{A}) \leq \operatorname{Adv}_{\mathcal{D}_{k}-\operatorname{SKerMDH}}\left(\mathcal{B}_{1}\right)+\operatorname{Adv}_{\left(\mathcal{M}^{\top}, h\right)-\mathrm{MDDH}}\left(\mathcal{B}_{2}\right)+1 / p$, where $h^{\prime}\left([\mathbf{M}]_{1},[\mathbf{N}]_{2},[\mathbf{P}]_{1},[\mathbf{Q}]_{2}\right)=(h(\mathbf{M}), \mathbf{N}, \mathbf{P}, \mathbf{Q})$.

2. When $\ell_{1}=\ell_{2}$, for any adversary $\mathcal{A}$ against the $h$-strong soundness of $\Pi_{k t-s u m}$ there exist adversaries $\mathcal{B}_{1}$ and $\mathcal{B}_{2}$ such that $\operatorname{Adv}_{\Pi_{k t-s u m, h^{\prime}}}(\mathcal{A}) \leq \operatorname{Adv}_{\mathcal{D}_{k} \text {-SKerMDH }}\left(\mathcal{B}_{1}\right)+\operatorname{Adv}_{\left(\mathcal{M}^{\top}, \mathcal{N}^{\top}, h\right)-\mathrm{MDDH}}\left(\mathcal{B}_{2}\right)+1 / p$, where $h^{\prime}\left([\mathbf{M}]_{1},[\mathbf{N}]_{2},[\mathbf{P}]_{1},[\mathbf{Q}]_{2}\right)=(h(\mathbf{M}, \mathbf{N}), \mathbf{P}, \mathbf{Q})$.

The proof is implicitly shown in [GR19, Lemma 15]. Essentially one can reduce to the knowledge transfer argument where we delete the zero columns of the matrix and rely on the linearity properties of the proofs of construction of Fig. 1.

${ }^{21}$ This part of the proof follows essentially the same lines of the first constant-size QA-NIZK arguments for linear spaces of Libert et al.[LPJY14] which were later simplified and generalized by Kiltz and Wee [KW15]. 